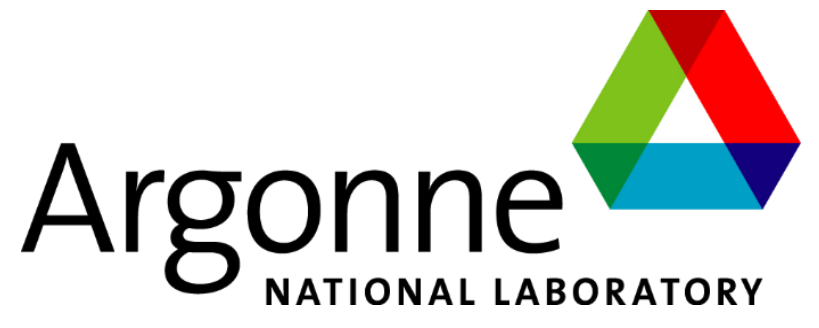

ANL/NED/17/2

\title{
Air Flow Modeling in the Wind Tunnel of the FHWA Aerodynamics Laboratory at Turner-Fairbank Highway Research Center
}

September 2017 


\title{
About Argonne National Laboratory
}

Argonne is a U.S. Department of Energy laboratory managed by UChicago Argonne, LLC under contract DE-AC02-06CH11357. The Laboratory's main facility is outside Chicago, at 9700 South Cass Avenue, Argonne, Illinois 60439. For information about Argonne and its pioneering science and technology programs, see www.anl.gov.

\section{DOCUMENT AVAILABILITY}

Online Access: U.S. Department of Energy (DOE) reports produced after 1991 and a growing number of pre-1991 documents are available free via DOE's SciTech Connect (http://www.osti.gov/scitech/)

\author{
Reports not in digital format may be purchased by the public from the \\ National Technical Information Service (NTIS): \\ U.S. Department of Commerce \\ National Technical Information Service \\ 5301 Shawnee Rd \\ Alexandra, VA 22312 \\ www.ntis.gov \\ Phone: (800) 553-NTIS (6847) or (703) 605-6000 \\ Fax: (703) 605-6900 \\ Email: orders@ntis.gov \\ Reports not in digital format are available to DOE and DOE contractors from the \\ Office of Scientific and Technical Information (OSTI): \\ U.S. Department of Energy \\ Office of Scientific and Technical Information \\ P.O. Box 62 \\ Oak Ridge, TN 37831-0062 \\ www.osti.gov \\ Phone: (865) 576-8401 \\ Fax: (865) 576-5728 \\ Email: reports@osti.gov
}

Disclaimer

This report was prepared as an account of work sponsored by an agency of the United States Government. Neither the United States Government nor any agency thereof, nor UChicago Argonne, LLC, nor any of their employees or officers, makes any warranty, express or implied, or assumes any legal liability or responsibility for the accuracy, completeness, or usefulness of any information, apparatus, product, or process disclosed, or represents that its use would not infringe privately owned rights. Reference herein to any specific commercial product, process, or service by trade name, trademark, manufacturer, or otherwise, does not necessarily constitute or imply its endorsement, recommendation, or favoring by the United States Government or any agency thereof. The views and opinions of document authors expressed herein do not necessarily state or reflect those of the United States Government or any agency thereof, Argonne National Laboratory, or UChicago Argonne, LLC. 


\section{Air Flow Modeling in the Wind Tunnel of the FHWA Aerodynamics Laboratory at Turner- Fairbank Highway Research Center}

by

M.A. Sitek, S.A. Lottes, C. Bojanowski

Transportation Research and Analysis Computing Center (TRACC)

Energy Systems Division, Argonne National Laboratory 
Table of Contents

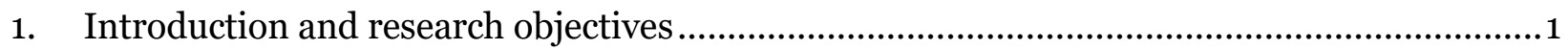

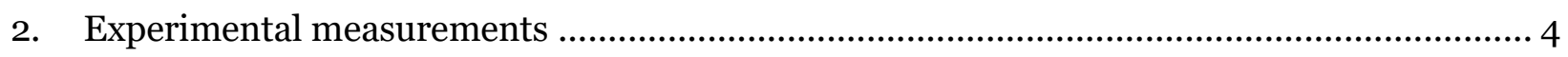

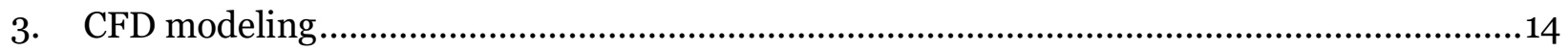

3.1. Description of the model with a rotating fan ....................................................... 17

3.2. Description of the model with rotating reference frame (RRF) ...................................18

3.3. Description of the simplified models ...........................................................................19

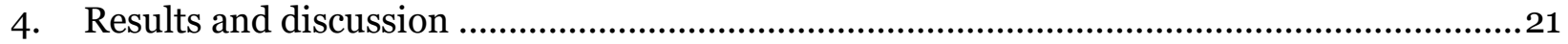

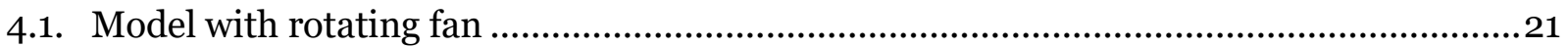

4.2. Model with rotating reference frame ...................................................................... 24

4.2.1. RANS, k- $\quad$ turbulence modeling ................................................................. 24

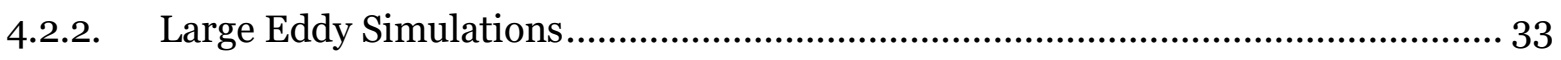

4.3. Results obtained with simplified models............................................................... 37

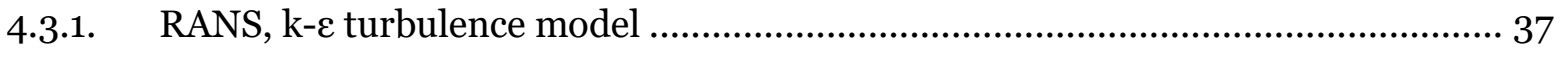

4.3.2. Large Eddy Simulations.............................................................................. 46

4.4. Other versions of the simplified model - one of the pressure outlet surfaces is closed .. 49

4.5. Other versions of the simplified model - non-uniform inlet parameter distributions .... 50

4.6. Other versions of the simplified model - back half of the room is cut off ......................661

4.7. Comparison of computational and experimental results .......................................... 62

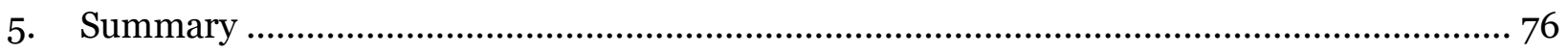

6. Recommendations for application of the models ........................................................77

6.1. Recommendations for turbulence models ............................................................... 78

6.2. Recommendations for geometry models .................................................................. 80

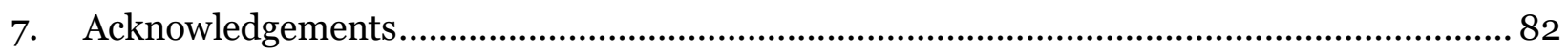

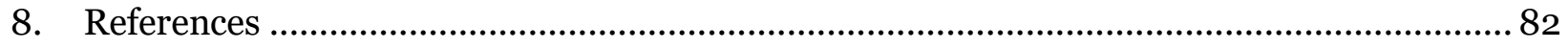

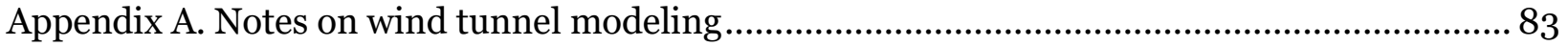

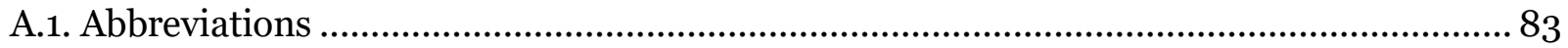

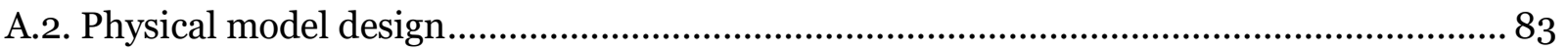

A.3. Preparing the domain for meshing ........................................................................ 83

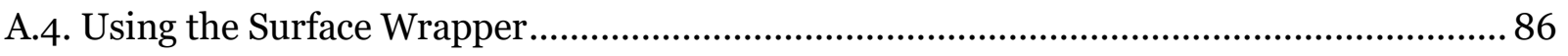

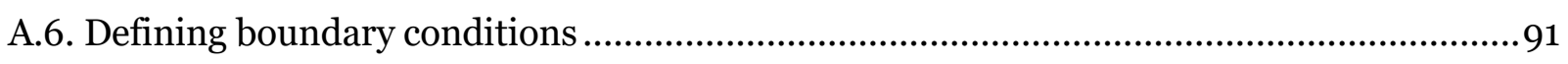

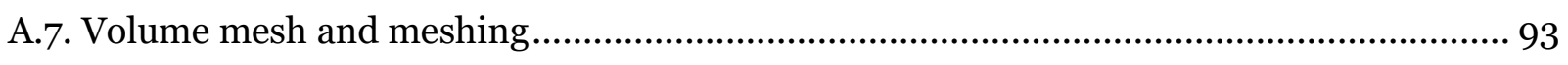

Air Flow Modeling in the Wind Tunnel of the FHWA Aerodynamics Laboratory at TFHRC $\quad$ Page I 


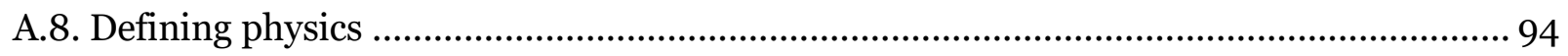

A.9. Defining fan rotation .................................................................................... 94

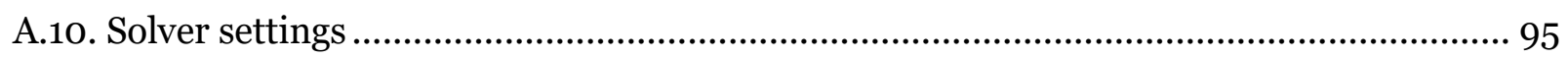

A.11. Introducing a test model to the wind tunnel domain................................................ 95

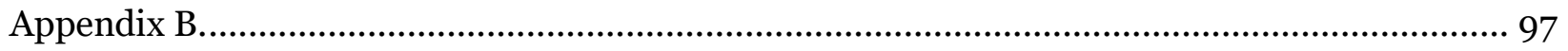




\section{List of Figures}

Figure 1-1: Aerodynamics Laboratory, exit of the wind tunnel and a turbulence generator on the right

Figure 1-2: Aerodynamics Laboratory, right hand side of the back of the room......................... 2

Figure 1-3: Aerodynamics Laboratory, one of the fan air intakes ............................................. 3

Figure 1-4: Aerodynamics Laboratory, furniture, equipment and the force balance.................... 3

Figure 2-1: Velocity vectors in the measuring points ....................................................... 4

Figure 2-2: A selection of measuring points ......................................................................... 4

Figure 2-3: Views of the wind tunnel extension, (a) side view, (b) front view. Locations of probes

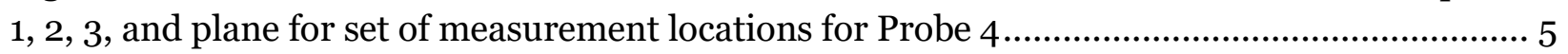

Figure 2-4: Velocity measurements at fan speed $117 \mathrm{rpm}$, (a) Probe 1, (b) Probe 2, (c) Probe 3, (d) Probe 4. Source: Turner-Fairbank Aerodynamics Laboratory............................................

Figure 2-5: Velocity measurements at probe 2 at the fan speed of (a) $117 \mathrm{rpm}$, (b) $292 \mathrm{rpm}$. Source: Turner-Fairbank Aerodynamics Laboratory............................................................ 8

Figure 2-6: Mean velocity measurement at a probe plane 4 plane vs. fan speed......................... 8

Figure 2-7: $\mathrm{X}$ and $\mathrm{Y}$ axis of the global coordinate system with positive angle direction. $\mathrm{Z}$ axis is assumed to point vertically and upwards .............................................................................. 9

Figure 2-8: History of velocity magnitude (a) at point 20, (b) at point 23, at fan speed of $219 \mathrm{rpm}$

Figure 2-9: History of velocity direction (a) at point 20, (b) at point 23, at fan speed of $219 \mathrm{rpm} 11$ Figure 2-10: History of velocity magnitude (a) at point 20, (b) at point 23, at a fan speed of 437

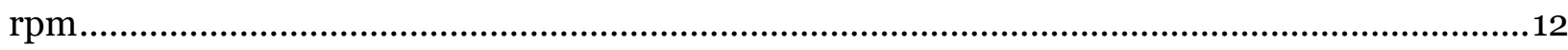

Figure 2-11: History of velocity direction (a) at point 20, (b) at point 23, at fan speed of $437 \mathrm{rpm}$

Figure 3-1: Geometry of the computational domain, (a) general view, (b) the test section in front of the wind tunnel with a turbulence generator on the right side..........................................15

Figure 3-2: A cross section through the wind tunnel ........................................................... 15

Figure 3-3: Volume mesh on the cross-section through the domain (a) top view, (b) side view.. 17 Figure 3-4: Volume mesh on the cross-section through the domain (a) top view, (b) side view, in LES simulation.................................................................................................................. 19 
Figure 3-5: A cross-section through the simplified model ................................................. 20

Figure 3-6: Further simplification of the model...............................................................20

Figure 4-1: Velocity in Y direction in points 20 and 23 vs. computational time ........................21

Figure 4-2: Distribution of velocity in $Y$ direction on planes: (a) $Y=-1.5$ in, (b) $Y=1.5$ in, (c) $Y=1 \mathrm{ft}$, (d) $\mathrm{Y}=2 \mathrm{ft}$. Model with rotating fan................................................................................... 23

Figure 4-3: Distribution of velocity in Y direction on a vertical plane of point 23. Model with rotating fan.

Figure 4-4: Velocity magnitude on (a) horizontal and (b) vertical planes. Model with rotating reference frame, RANS solver.

Figure 4-5: Distribution of (a) normal velocity, (b) inertial porous resistance of the screens for $\alpha$ depending on local velocity values. Model with rotating reference frame, RANS solver.

Figure 4-6: Velocity magnitude at points 20 and 23. Model with rotating reference frame, RANS solver. 28

Figure 4-7: The distribution of velocity in $Y$ direction on vertical planes: (a) $Y=-1.5$ in, (b) $Y=1.5$ in, (c) $\mathrm{Y}=1 \mathrm{ft}$, (d) $\mathrm{Y}=2 \mathrm{ft}$. Model with rotating reference frame, RANS solver. 30

Figure 4-8: Turbulence intensity distribution on planes: (a) $Y=-1.5$ in, (b) $Y=1.5$ in, (c) $Y=1 \mathrm{ft}$, (d) $\mathrm{Y}=2 \mathrm{ft}$. Model with rotating reference frame, RANS solver......

Figure 4-9: Relationship between the average normal velocity at the last screen in the wind tunnel and the fan speed. A linear fit was shown. Model with rotating reference frame, RANS solver.

Figure 4-10: Velocity magnitude on a) horizontal plane, b) vertical plane. Model with rotating reference frame, LES solver.

Figure 4-11: Velocity in Y direction at points 20 and 23. Model with rotating reference frame, LES solver.

Figure 4-12: The distribution of velocity in $Y$ direction on planes: (a) $Y=-1.5$ in, (b) $Y=1.5$ in, (c) $\mathrm{Y}=1 \mathrm{ft}$, (d) $\mathrm{Y}=2 \mathrm{ft}$. Model with rotating reference frame, LES solver.

Figure 4-13: The distribution of velocity in $\mathrm{Y}$ direction on a vertical plane of (a) point 20, (b) point 23. Model with rotating reference frame, LES solver.

Figure 4-14: Velocity vs iterations at points 20 and 23 during the simulation. Model with simplified geometry, RANS solver.

Figure 4-15: Velocity magnitude distribution on (a) horizontal plane, (b) vertical plane. Model with simplified geometry, RANS solver. 38 
Figure 4-16: The distribution of velocity in $Y$ direction on planes: (a) $Y=-1.5$ in, (b) $Y=1.5$ in, (c) $\mathrm{Y}=1 \mathrm{ft}$, (d) $\mathrm{Y}=2 \mathrm{ft}$. Model with simplified geometry, RANS solver.

Figure 4-17: Turbulence intensity distribution on planes: (a) $Y=-1.5$ in, (b) $Y=1.5$ in, (c) $Y=1 \mathrm{ft}$, (d) $\mathrm{Y}=2 \mathrm{ft}$. Model with simplified geometry, RANS solver.

Figure 4-18: Average velocity at Probe 4 vs. fan speed. Model with simplified geometry, RANS solver.

Figure 4-19: Velocity at Probe 1 vs. fan speed. Model with simplified geometry, RANS solver... 44

Figure 4-20: Velocity at Probe 2 vs. fan speed. Model with simplified geometry, RANS solver.. 44

Figure 4-21: Velocity at Probe 3 vs. fan speed. Model with simplified geometry, RANS solver. . 45

Figure 4-22: Velocity magnitude distribution on (a) horizontal, (b) vertical planes. Model with simplified geometry, LES solver.

Figure 4-23: The distribution of velocity in $Y$ direction on vertical planes (a) $Y=-1.5$ in, (b) $Y=1.5$ in, (c) $\mathrm{Y}=1 \mathrm{ft}$, (d) $\mathrm{Y}=2 \mathrm{ft}$. Model with simplified geometry, LES solver. 48

Figure 4-24: Velocity in Y direction at points 20 and 23 vs. simulation time. Model with simplified geometry, LES solver.

Figure 4-25: Velocity magnitude distribution (a) on a horizontal plane, (b) on a vertical plane, in the model with left hand side pressure outlet blocked 50

Figure 4-26: Velocity magnitude distribution (a) on a horizontal plane, (b) on a vertical plane, in the model with right hand side pressure outlet blocked 50

Figure 4-27: Inlet velocity boundary condition input parameters at fan speed $48 \mathrm{rpm}$ : (a) X velocity, (b) Y velocity, (c) Z velocity, (d) turbulence intensity, (e) turbulent viscosity ratio ..... 53

Figure 4-28: Inlet velocity boundary condition input parameters at fan speed $437 \mathrm{rpm}$ : (a) X velocity, (b) Y velocity, (c) Z velocity, (d) turbulence intensity, (e) turbulent viscosity ratio ...... 56

Figure 4-29: Side view of the velocity magnitude distribution at fan speed $48 \mathrm{rpm}$ on a vertical plane for models: (a) with rotating reference frame, (b) simplified geometry with uniform inlet velocity, (c) simplified geometry with non-uniform inlet velocity .

Figure 4-30: Top view of the velocity magnitude distribution at fan speed $48 \mathrm{rpm}$ on a horizontal plane for models: (a) with rotating reference frame, (b) simplified geometry with uniform inlet velocity, (c) simplified geometry with non-uniform inlet velocity....

Figure 4-31: Side view of the velocity magnitude distribution at fan speed $437 \mathrm{rpm}$ on a vertical plane for models: (a) with rotating reference frame, (b) simplified geometry with uniform inlet velocity, (c) simplified geometry with non-uniform inlet velocity . 
Figure 4-32: Top view of the velocity magnitude distribution at fan speed $437 \mathrm{rpm}$ on a horizontal plane for models: (a) with rotating reference frame, (b) simplified geometry with uniform inlet velocity, (c) simplified geometry with non-uniform inlet velocity 60

Figure 4-33: Velocity in $\mathrm{Y}$ direction at points 20 and 23 vs iteration number 61

Figure 4-34: Velocity magnitude distribution on (a) horizontal, (b) vertical planes .................. 62

Figure 4-35: Comparison of velocity magnitude along lines a) I, b) II, c) III ........................... 65

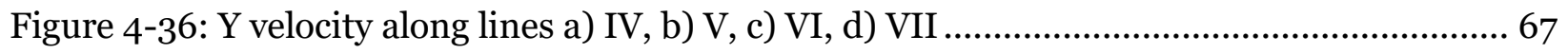

Figure 4-37. Comparison of experimental and computational velocities in the flow direction at probes 1, 2, and 3, at fan speed (a) $117 \mathrm{rpm}$, (b) $223 \mathrm{rpm}$, (c) $292 \mathrm{rpm}$. Model with rotating reference frame, RANS solver.

Figure 4-38. Comparison of experimental and computational velocity magnitudes at probe plane 4 (a) $117 \mathrm{rpm}$, (b) $223 \mathrm{rpm}$, (c) $292 \mathrm{rpm}$. Model with rotating reference frame, RANS solver .... 70

Figure 4-39. Computational velocity components at probe plane 4 (a) $117 \mathrm{rpm}$, (b) $223 \mathrm{rpm}$, (c) $292 \mathrm{rpm}$. Model with rotating reference frame, RANS solver. 72

Figure 4-40. Comparison of experimental and computational velocities in the flow direction at probes 1, 2, and 3, at fan speed (a) $117 \mathrm{rpm}$, (b) $223 \mathrm{rpm}$, (c) $292 \mathrm{rpm}$. Model with simplified geometry, RANS solver.

Figure 4-41. Comparison of experimental and computational velocity magnitudes in the flow direction at probe plane 4, at fan speed (a) $117 \mathrm{rpm}$, (b) $223 \mathrm{rpm}$, (c) $292 \mathrm{rpm}$. Model with simplified geometry, RANS solver.

Figure 4-42. Computational velocity components in the flow direction at probe plane 4, at fan speed (a) $117 \mathrm{rpm}$, (b) $223 \mathrm{rpm}$, (c) $292 \mathrm{rpm}$. Model with simplified geometry, RANS solver.... 76 


\section{List of Tables}

Table 2-1: Average velocity and its standard deviation at point 20 ........................................13

Table 2-2: Average velocity and its standard deviation at point 23 .........................................14

Table 3-1: Run times and clock times for the performed simulations .....................................16

Table 4-1: Average velocity in Y direction at points 20 and 23 ..........................................21

Table 4-2: Pressure drop across screens for two different formulations of porous inertial resistance. Numbering of the screens in Figure 3-2. Model with rotating reference frame, RANS

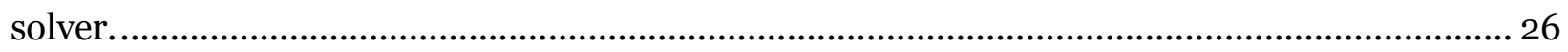

Table 4-3: Average flow velocity obtained in laboratory measurements at various fan speeds... 45

Table 4-4: A comparison of velocity values at Probe 1, 2, and 3 between the measurements and results obtained with the use of the model with simplified geometry, RANS solver.

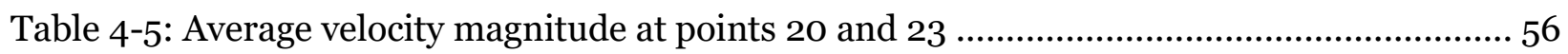

Table 4-6: Average normal velocity on the wind tunnel screens ............................................ 63 


\section{Introduction and research objectives}

Computational fluid dynamics (CFD) modeling is widely used in industry for design and in the research community to support, compliment, and extend the scope of experimental studies. Analysis of transportation infrastructure using high performance cluster computing with CFD and structural mechanics software is done at the Transportation Research and Analysis Computing Center (TRACC) at Argonne National Laboratory. These resources, available at TRACC, were used to perform advanced three-dimensional computational simulations of the wind tunnel laboratory at the Turner-Fairbank Highway Research Center (TFHRC). The goals were to verify the CFD model of the laboratory wind tunnel and then to use versions of the model to provide the capability to (1) perform larger parametric series of tests that can be easily done in the laboratory with available budget and time, (2) to extend testing to wind speeds that cannot be achieved in the laboratory, and (3) to run types of tests that are very difficult or impossible to run in the laboratory. Modern CFD software has many physics models and domain meshing options. Models, including the choice of turbulence and other physics models and settings, the computational mesh, and the solver settings, need to be validated against measurements to verify that the results are sufficiently accurate for use in engineering applications. The wind tunnel model was built and tested, by comparing to experimental measurements, to provide a valuable tool to perform these types of studies in the future as a complement and extension to TFHRC's experimental capabilities.

Wind tunnel testing at TFHRC is conducted in a subsonic open-jet wind tunnel with a $1.83 \mathrm{~m}$ (6 foot) by $1.83 \mathrm{~m}$ (6 foot) cross section. A three component dual force-balance system is used to measure forces acting on tested models, and a three degree of freedom suspension system is used for dynamic response tests. Pictures of the room are shown in Figure 1-1 to Figure 1-4.

A detailed CAD geometry and CFD model of the wind tunnel laboratory at TFHRC was built and tested. Results were compared against experimental wind velocity measurements at a large number of locations around the room. This testing included an assessment of the air flow uniformity provided by the tunnel to the test zone and assessment of room geometry effects, such as influence of the proximity to the room walls, the non-symmetrical position of the tunnel in the room, and the influence of the room setup on the air flow in the room. This information is useful both for simplifying the computational model and in deciding whether or not moving, or removing, some of the furniture or other movable objects in the room will change the flow in the test zone. 


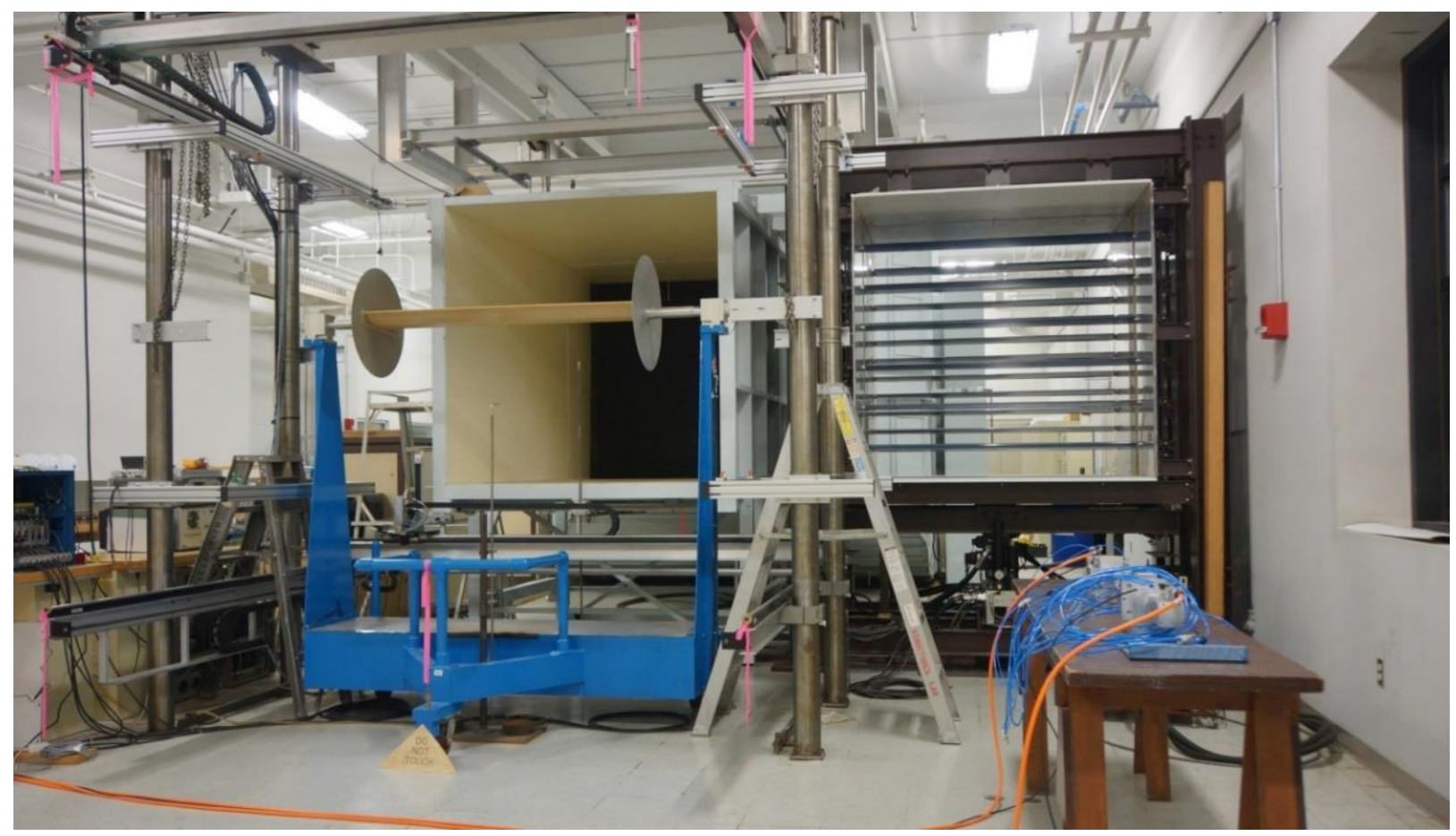

Figure 1-1: Aerodynamics Laboratory, exit of the wind tunnel and a turbulence generator on the right

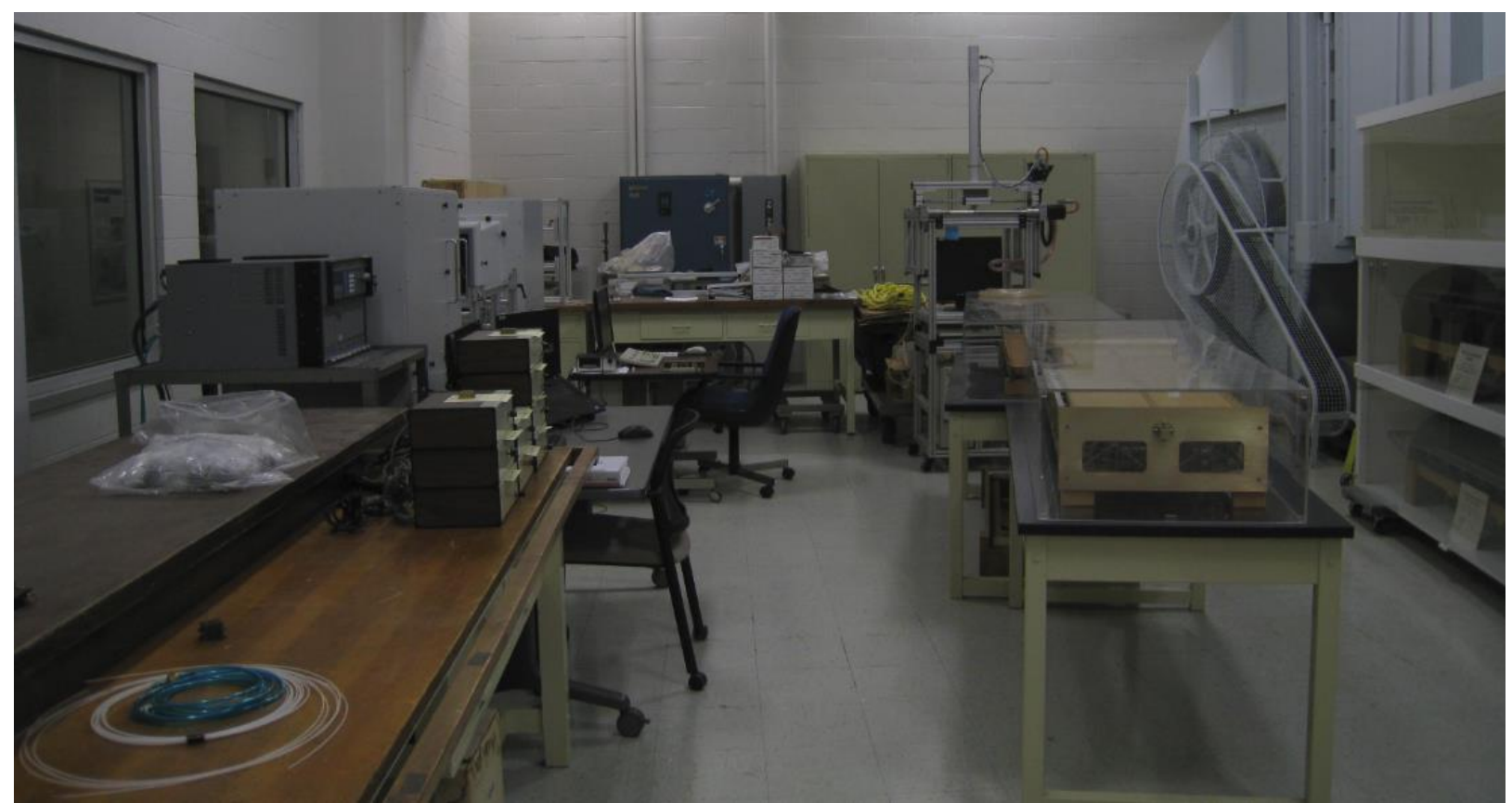

Figure 1-2: Aerodynamics Laboratory, right hand side of the back of the room 


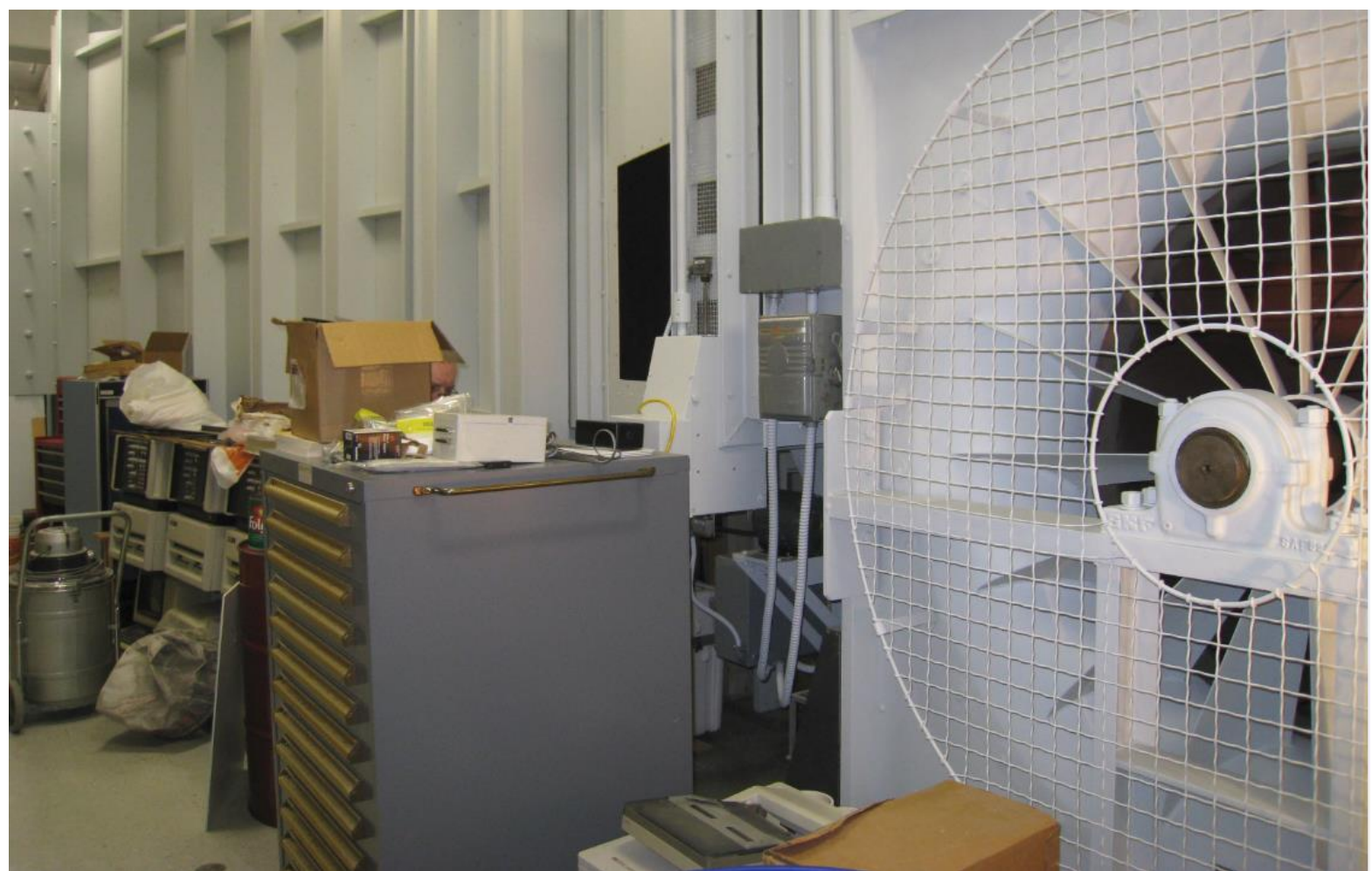

Figure 1-3: Aerodynamics Laboratory, one of the fan air intakes

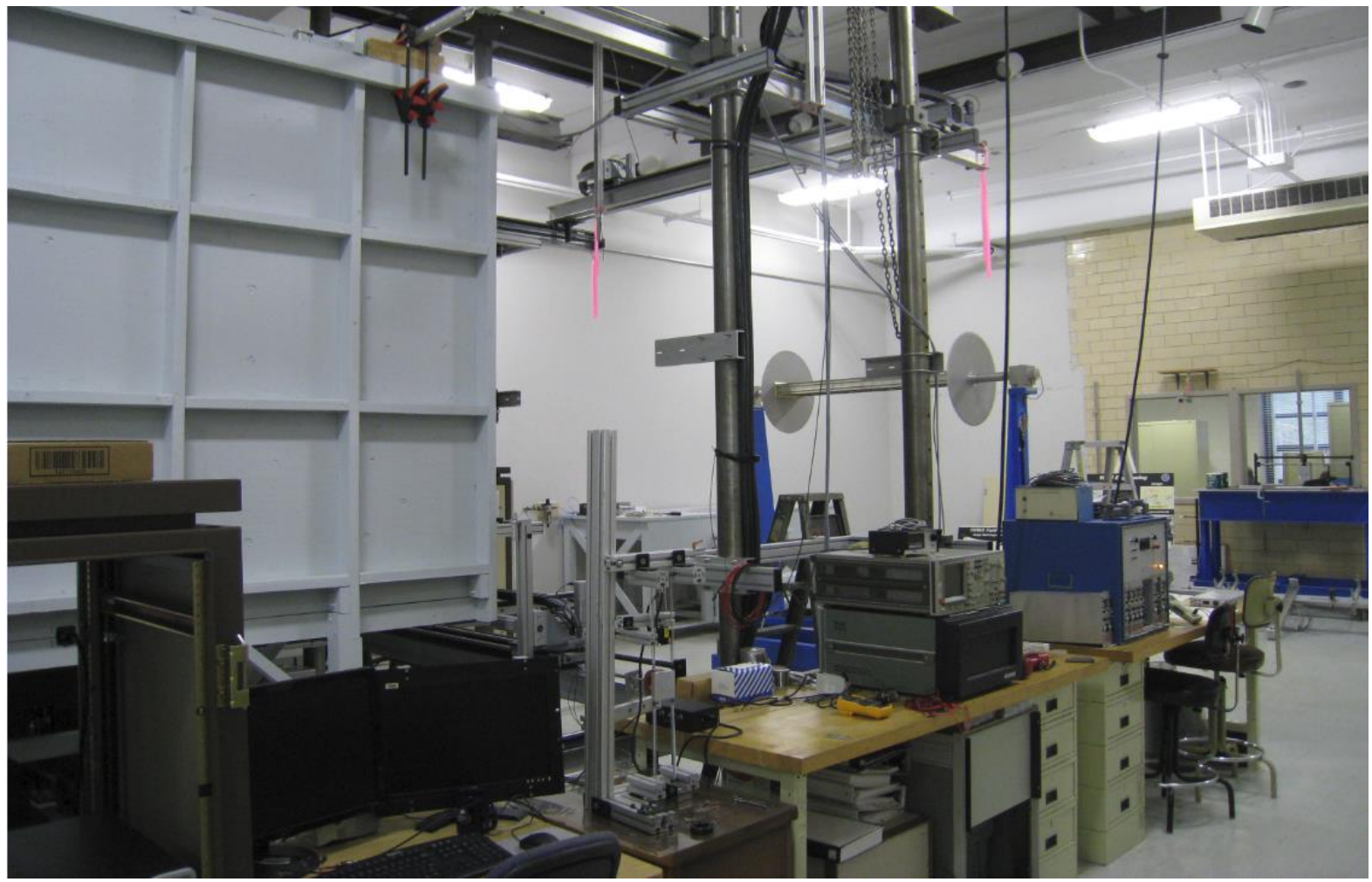

Figure 1-4: Aerodynamics Laboratory, furniture, equipment and the force balance 


\section{Experimental measurements}

Measurements of velocity magnitude and direction were conducted at various locations in the laboratory room, along the sides of the wind tunnel and in the jet region, $2.13 \mathrm{~m}$ ( 7 feet) above the floor. The velocity vectors of time-averaged velocities are illustrated in Figure 2-1.

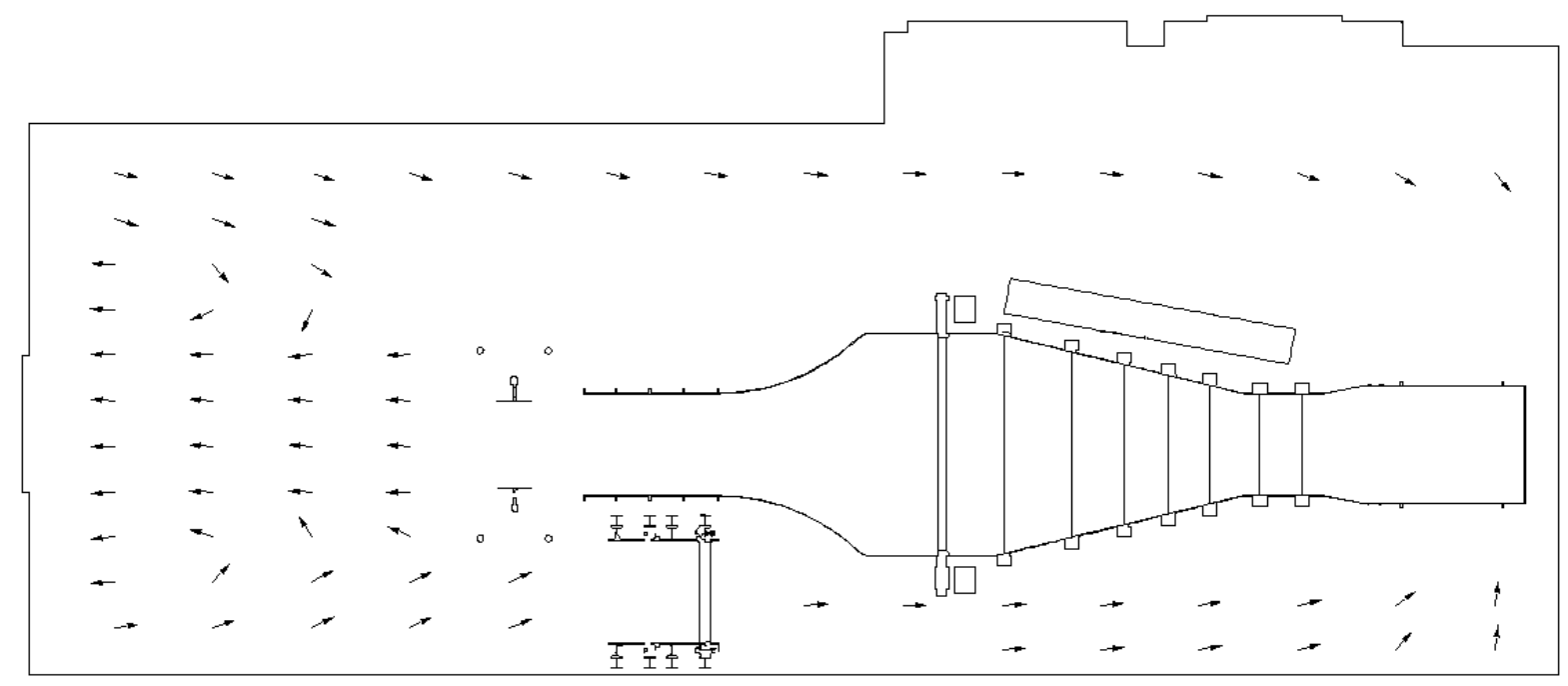

Figure 2-1: Velocity vectors in the measuring points

The time average of measured velocity at these points was compared to velocity distributions along probe lines obtained in the simulations. The probe lines used to monitor velocity in the simulations appear as brown dots in Figure 2-2.

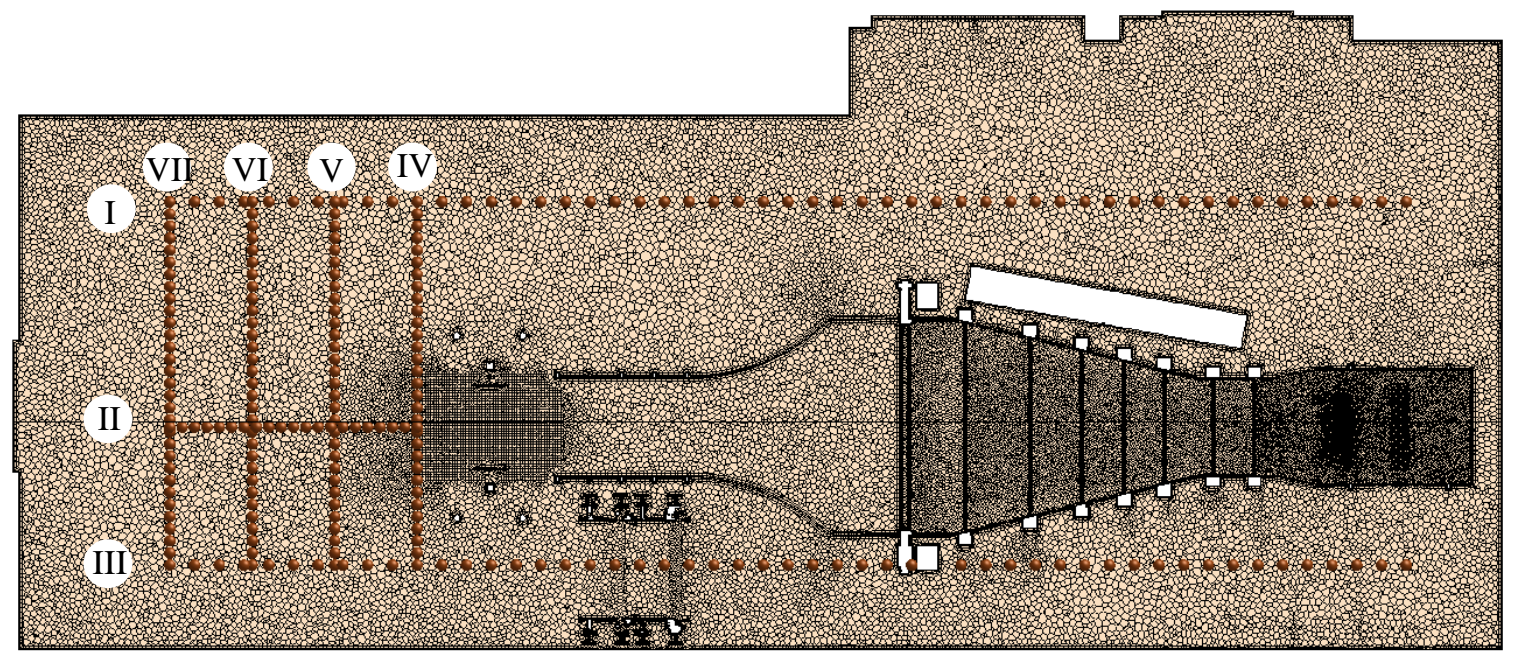

Figure 2-2: A selection of measuring points 
Velocity was also measured in three probes on the vertical centerline of the wind tunnel exit, and on plane 4, whose locations are shown in Figure 2-3. The measurements were taken while a pitot tube is moved on plane 4, every 6 in in each direction. Six speeds of the fan were taken into account: 117, 175, 233, 263, 292, and $321 \mathrm{rpm}$. As an example, probe 1, 2, and 3 measurements at fan speed $117 \mathrm{rpm}$ are illustrated in Figure 2-4. Small differences can be noticed in the readings when moving probe 4, due to the current location of the pitot tube. The biggest differences were noted for probe point 2, which is located outside of the wind tunnel.

(a)

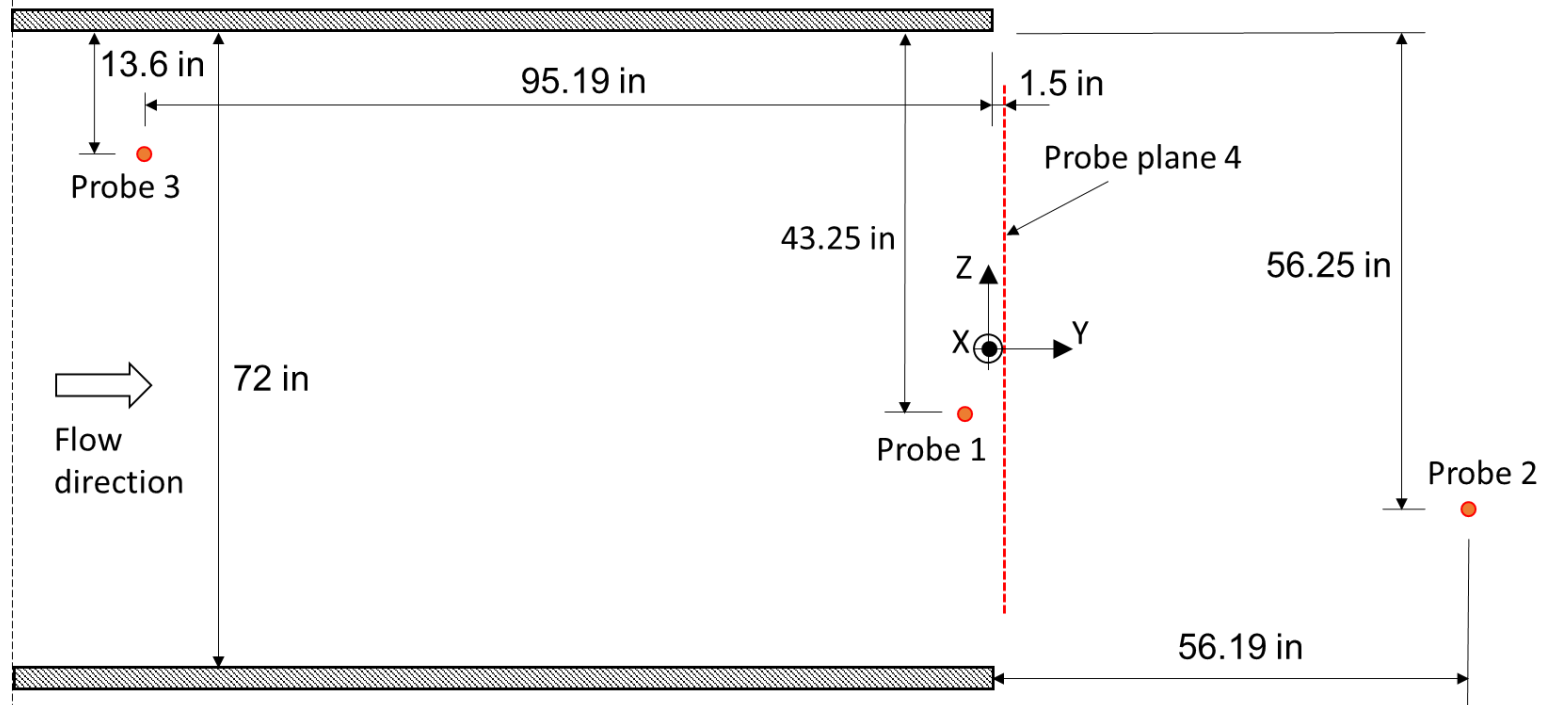

(b)

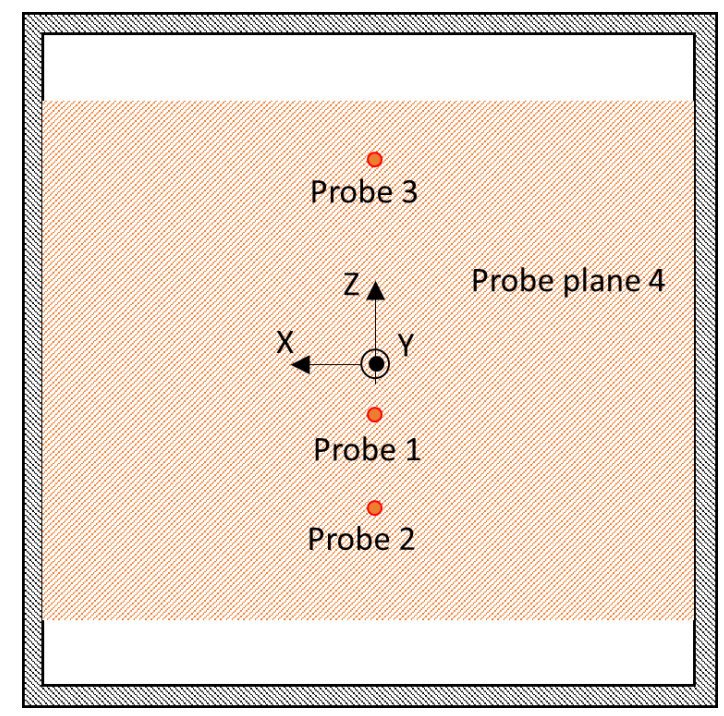

Figure 2-3: Views of the wind tunnel extension, (a) side view, (b) front view. Locations of probes 1, 2, 3, and plane for set of measurement locations for Probe 4 
(a)

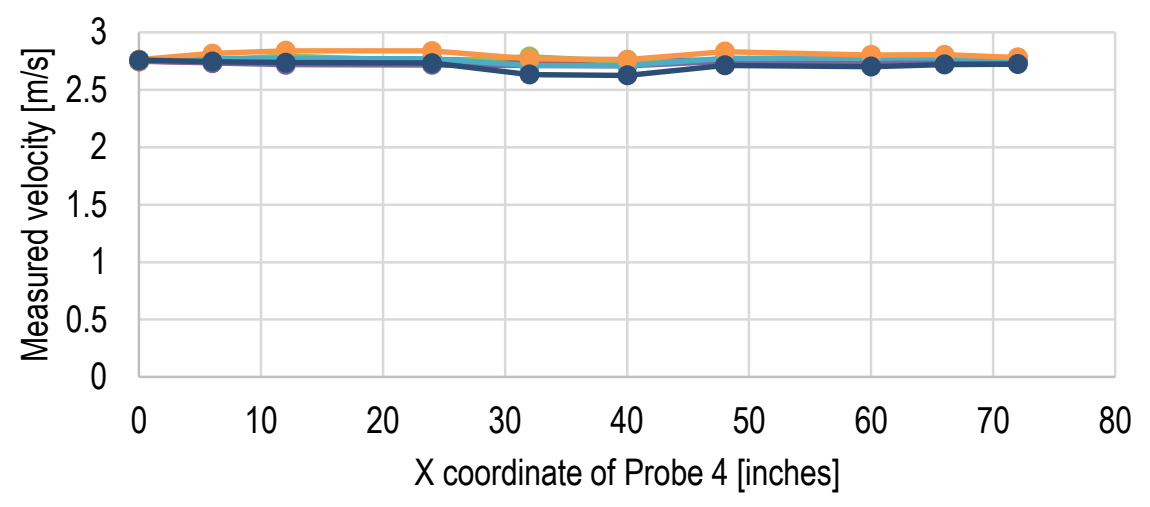

$-Z=6-Z=12-\mathrm{Z}=24-\mathrm{Z}=36-\mathrm{Z}=48-\mathrm{Z}=60-\mathrm{Z}=66$

(b)

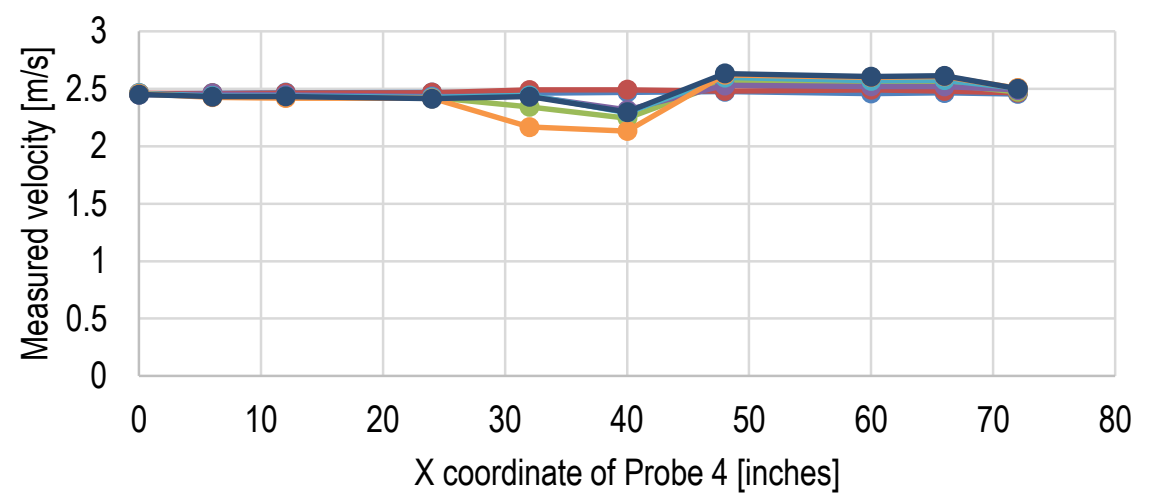

$-Z=6-Z=12-Z=24-Z=36-Z=48-Z=60-Z=66$

(c)

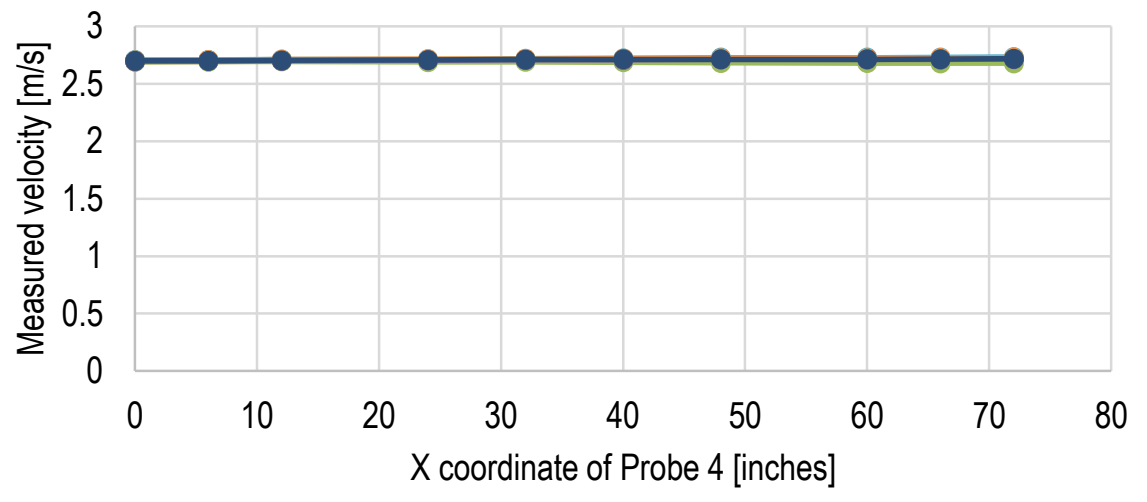

$\multimap-Z=6 \multimap-Z=12 \multimap-Z=24 \multimap-Z=36 \multimap-Z=48 \multimap-Z=60 \multimap-Z=66$ 
(d)

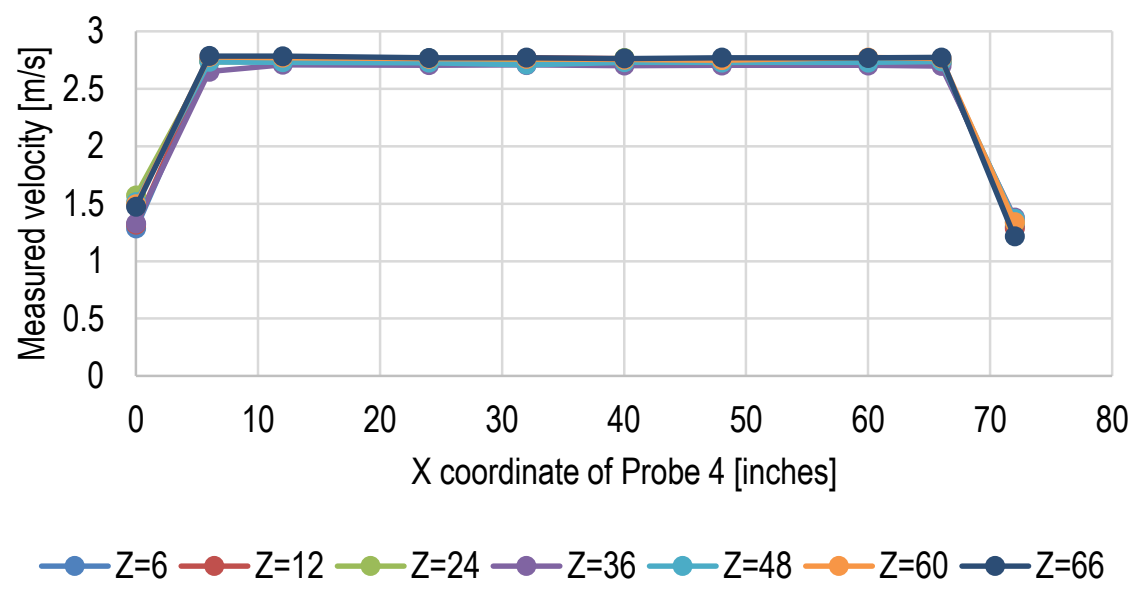

Figure 2-4: Velocity measurements at fan speed $117 \mathrm{rpm}$, (a) Probe 1, (b) Probe 2, (c) Probe 3, (d) Probe 4.

Source: Turner-Fairbank Aerodynamics Laboratory.

Probe measurements at Probe 2 for two different flow velocities are illustrated in Figure 2-5. They show differences in measured velocities, which are influenced by the location of probe 4 in the flow. Probe point 2 is the most influenced measuring point, as it is located furthest away from the wind tunnel exit. When probe 4 is located at a point with coordinates $\mathrm{X}=1.75 \mathrm{in}, \mathrm{Y}=4 \mathrm{O}$ in, $\mathrm{Z}=6 \mathrm{O}$ in i.e. in front of the probe 2, the variation is the highest. It also slightly increases with fan speed, e.g. for fan speed $117 \mathrm{rpm}$, the maximum relative difference is $13.6 \%$, and for fan speed $292 \mathrm{rpm}$, it is equal $16.5 \%$. Note that probe 4 is not normally present during production wind tunnel tests.

(a)

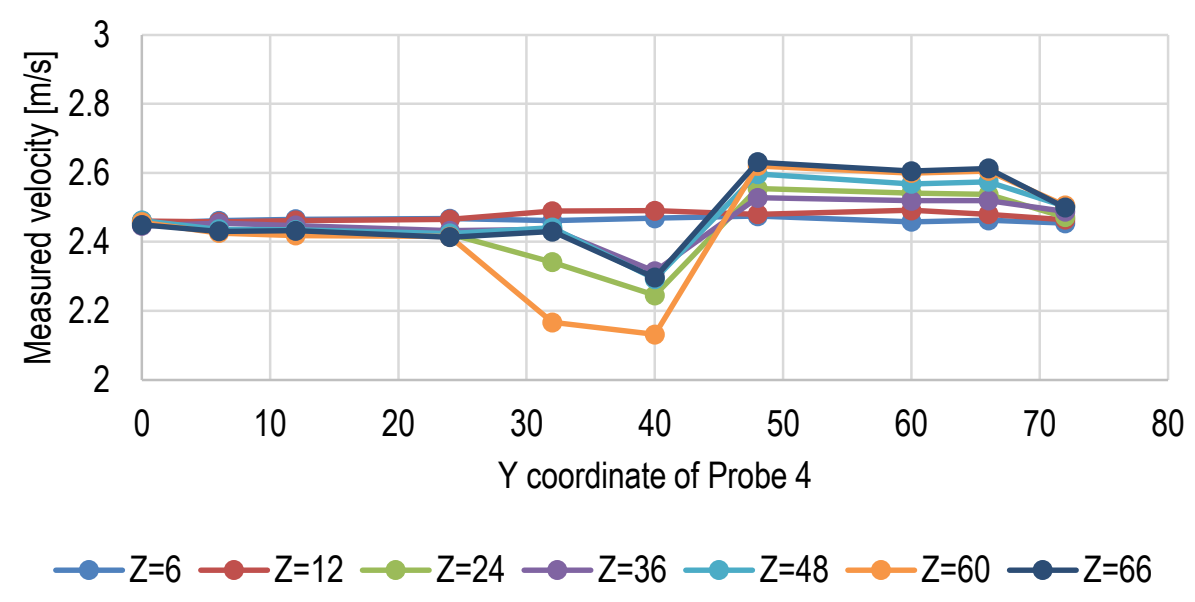


(b)

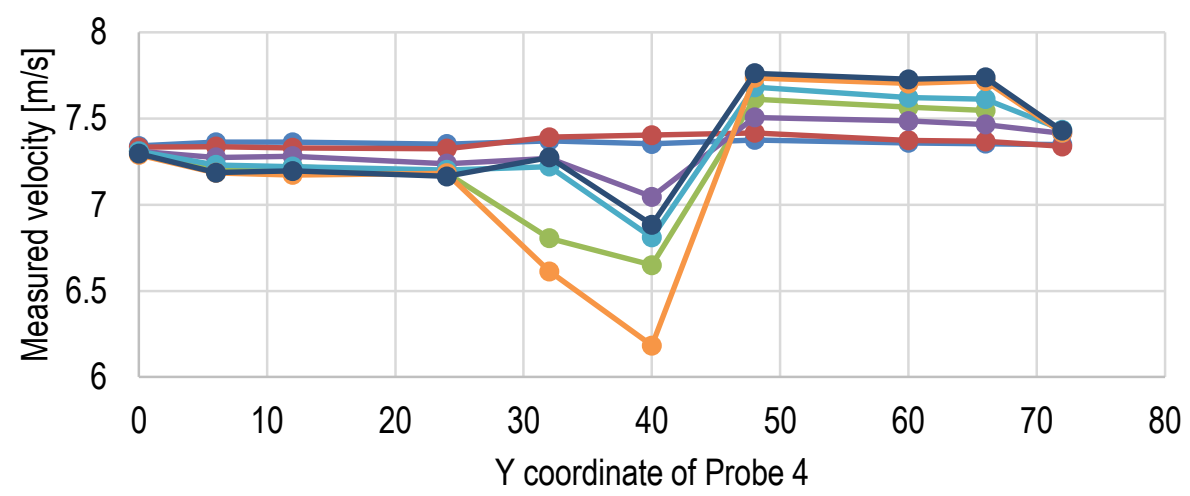

$\multimap-\mathrm{Z}=6 \rightarrow \mathrm{Z}=12 \multimap \mathrm{Z}=24 \rightarrow \mathrm{Z}=36 \multimap \mathrm{Z}=48 \rightarrow \mathrm{Z}=60 \multimap \mathrm{Z}=66$

Figure 2-5: Velocity measurements at probe 2 at the fan speed of (a) $117 \mathrm{rpm}$, (b) $292 \mathrm{rpm}$. Source:

Turner-Fairbank Aerodynamics Laboratory.

Average velocity for measurements at Probe 4 at different fan speeds was calculated by taking into account 6 inner measuring points (see Figure 2-4 d). They are combined for variable fan speed in Figure 2-6. In this region the flow was almost uniform, with the differences lower than $5 \%$. These values were later used to validate computational results. The measuring points located on the perimeter experience much lower velocity, which was a result of the boundary layer developing at the wind tunnel extension wall.

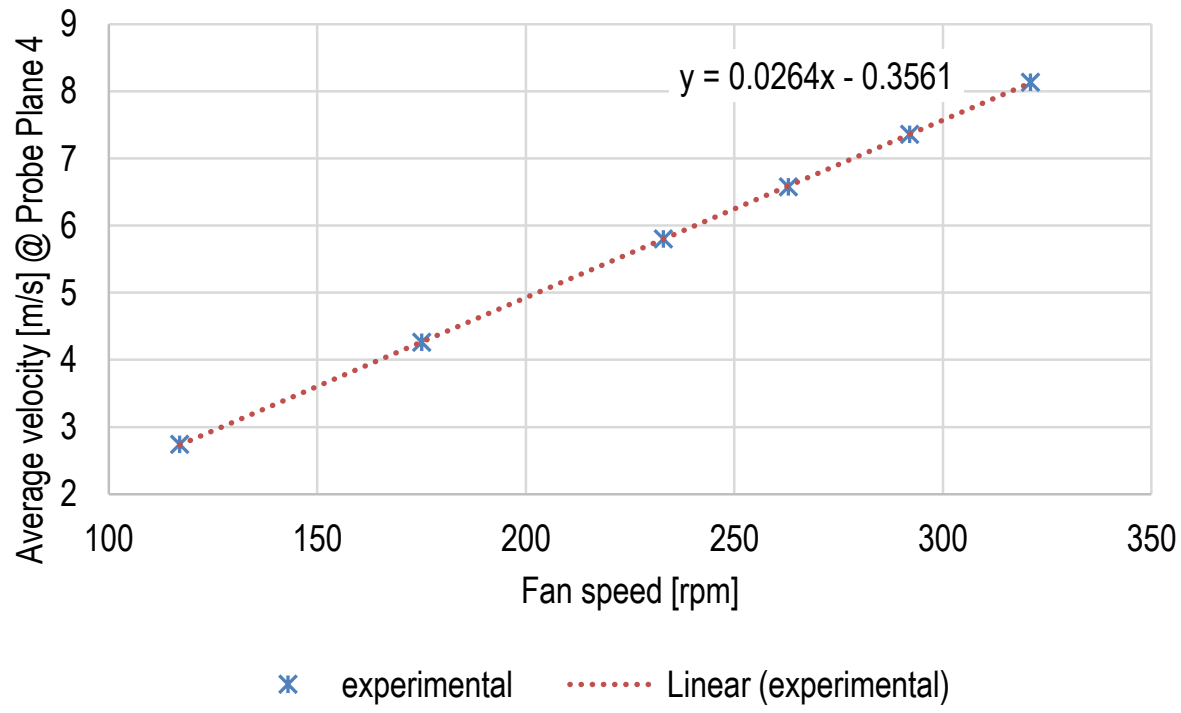

Figure 2-6: Mean velocity measurement at a probe plane 4 plane vs. fan speed 
Figure 2-8 and Figure 2-10 show oscillations of wind velocity at points 20 and 23, and Figure 2-9 and Figure 2-11 show the oscillations of the velocity direction. It was assumed that the angle equal to zero degrees represents a flow along $\mathrm{Y}$ axis. Figure 2-7 shows the $\mathrm{X}$ and $\mathrm{Y}$ axis of the global coordinate system with positive angle direction. $Z$ axis is assumed to point vertically and upwards. The locations of points 20 and 23 are shown in Figure 2-2. These points were positioned in the jet, but outside of the testing zone. Point number 20 was located $2.58 \mathrm{~m}$ away from the exit and point number 23 was $7.15 \mathrm{~m}$ away from it. It was observed that variations are higher at point 23, which is closer to the back wall. The average values and standard deviations are combined in Table 2-1 and Table 2-2.

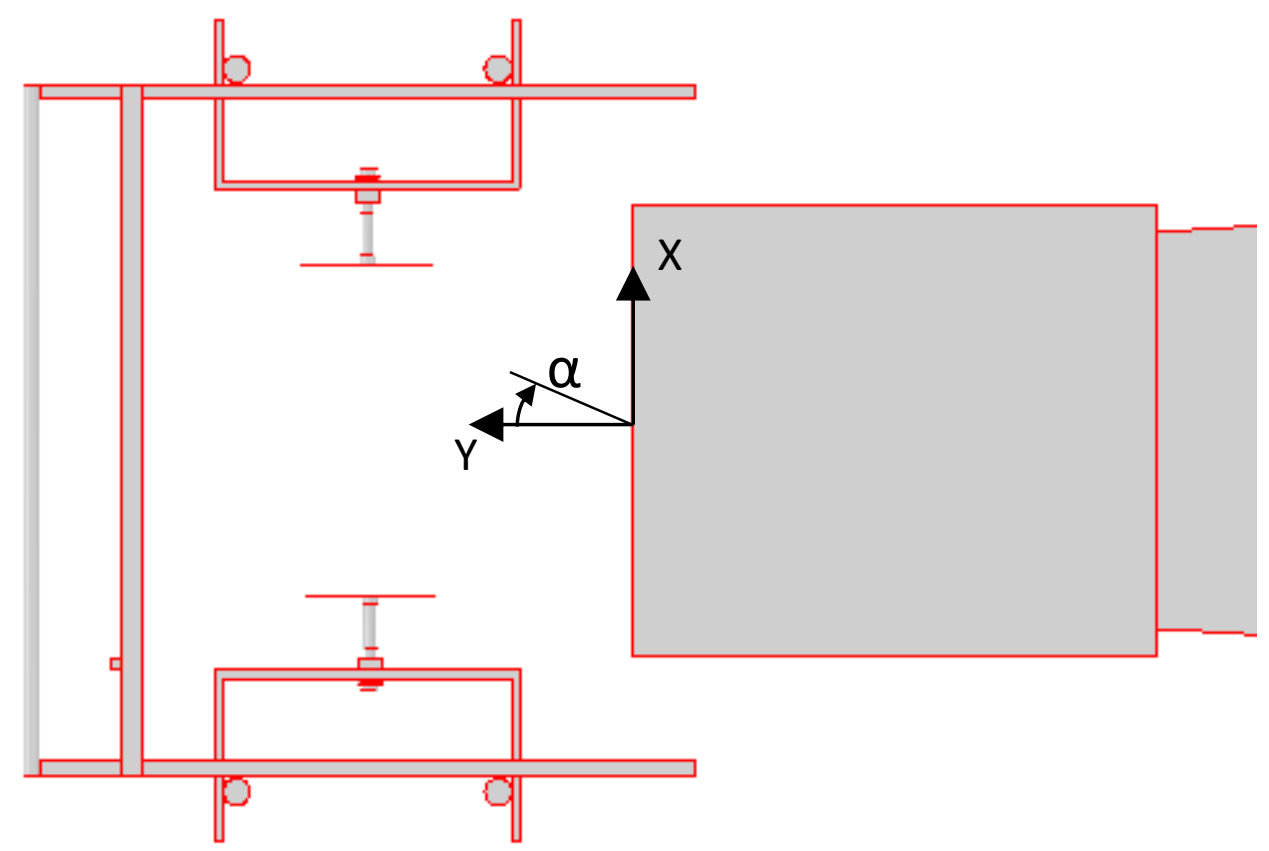

Figure 2-7: $\mathrm{X}$ and $\mathrm{Y}$ axis of the global coordinate system with positive angle direction. $\mathrm{Z}$ axis is assumed to point vertically and upwards 
(a)

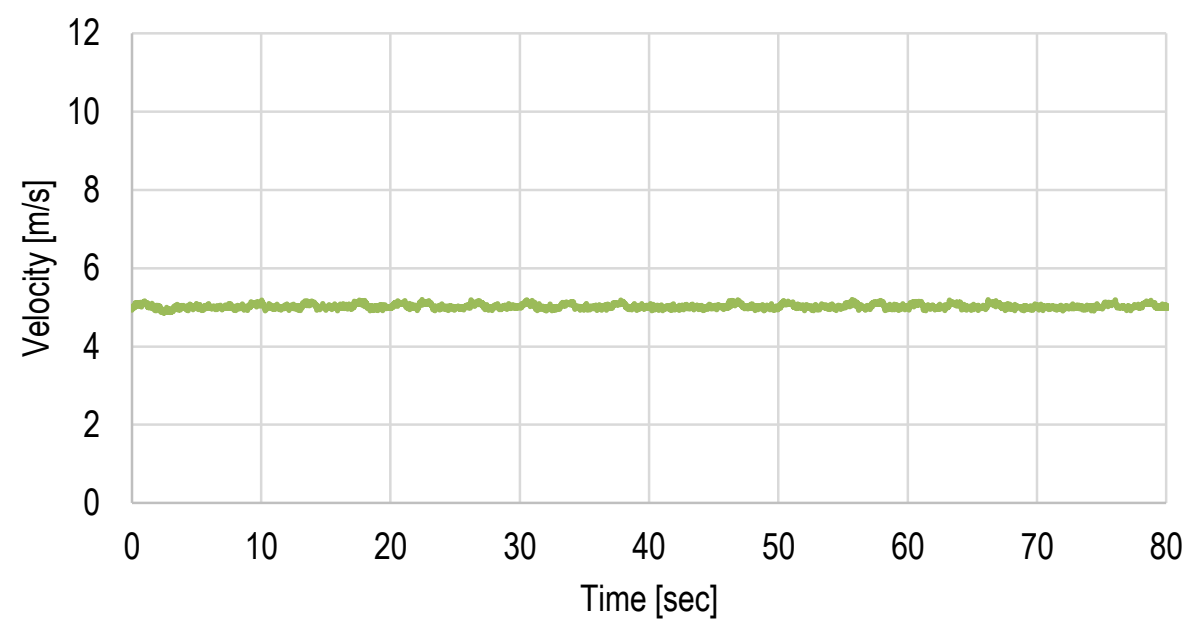

(b)

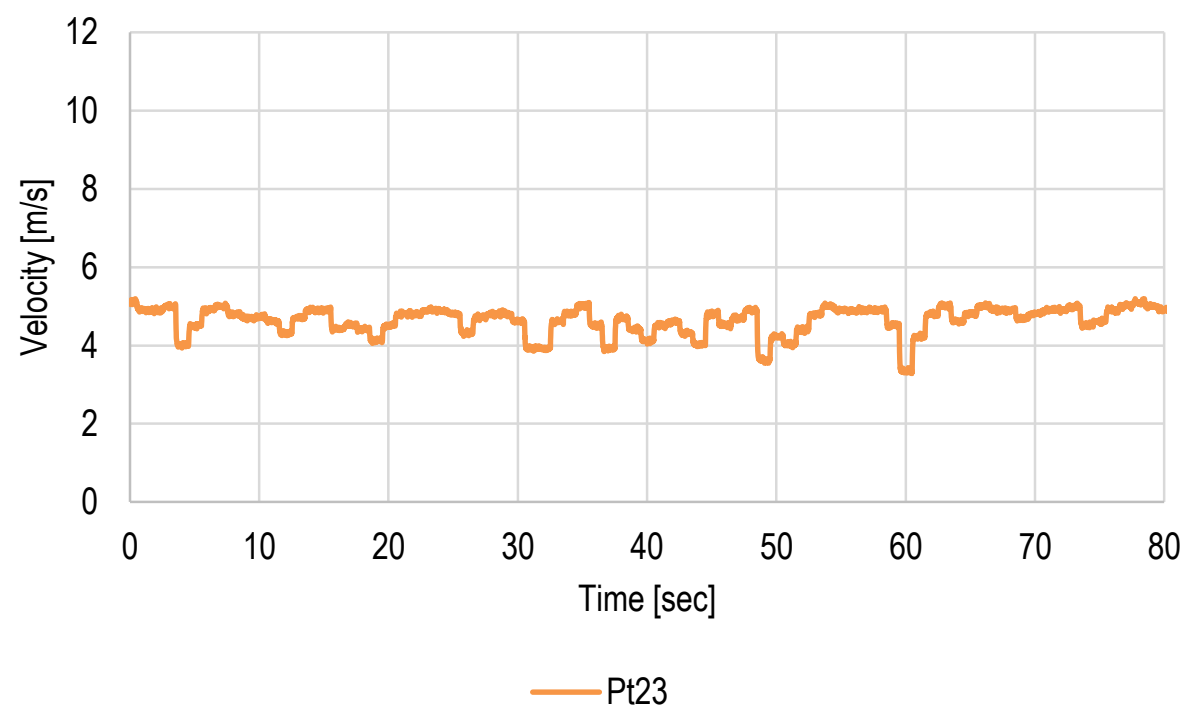

Figure 2-8: History of velocity magnitude (a) at point 20, (b) at point 23, at fan speed of $219 \mathrm{rpm}$ 
(a)

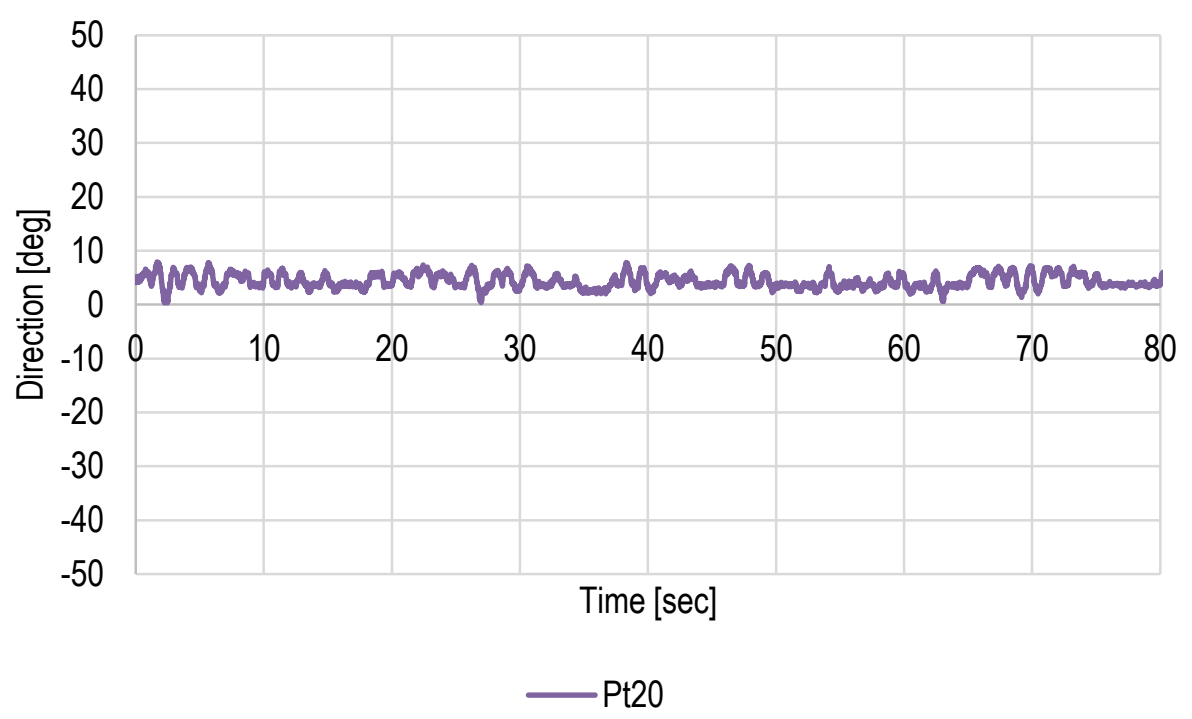

(b)

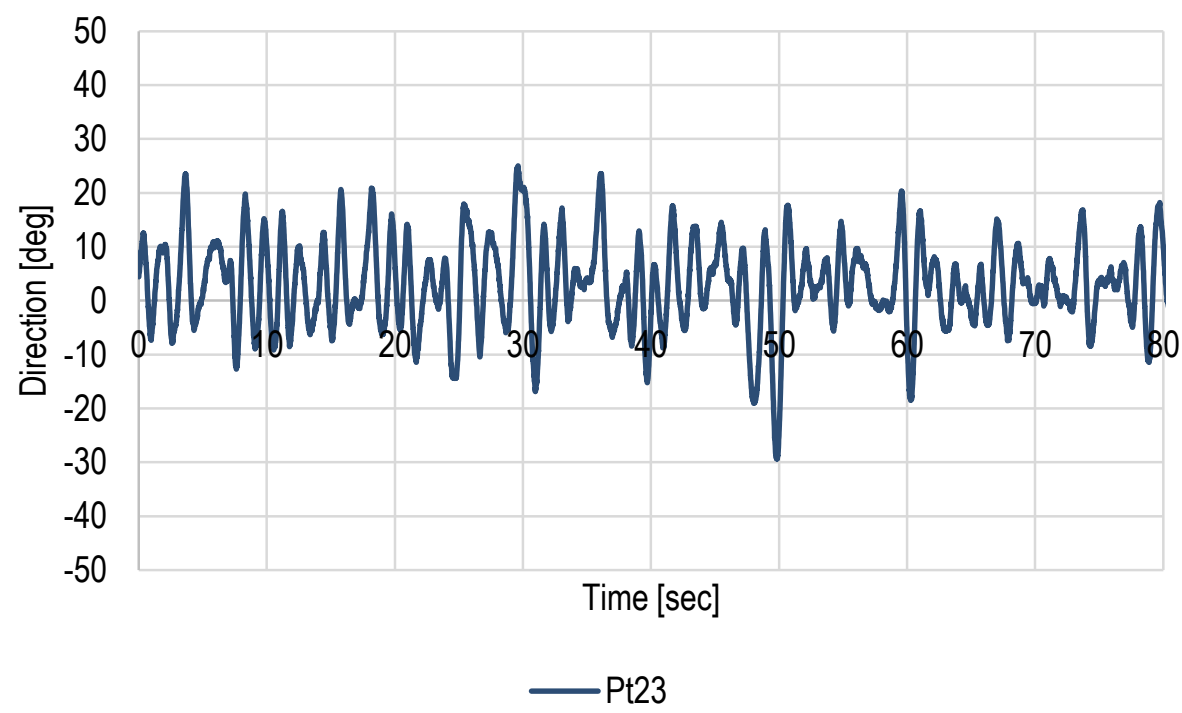

Figure 2-9: History of velocity direction (a) at point 20, (b) at point 23, at fan speed of $219 \mathrm{rpm}$ 
(a)

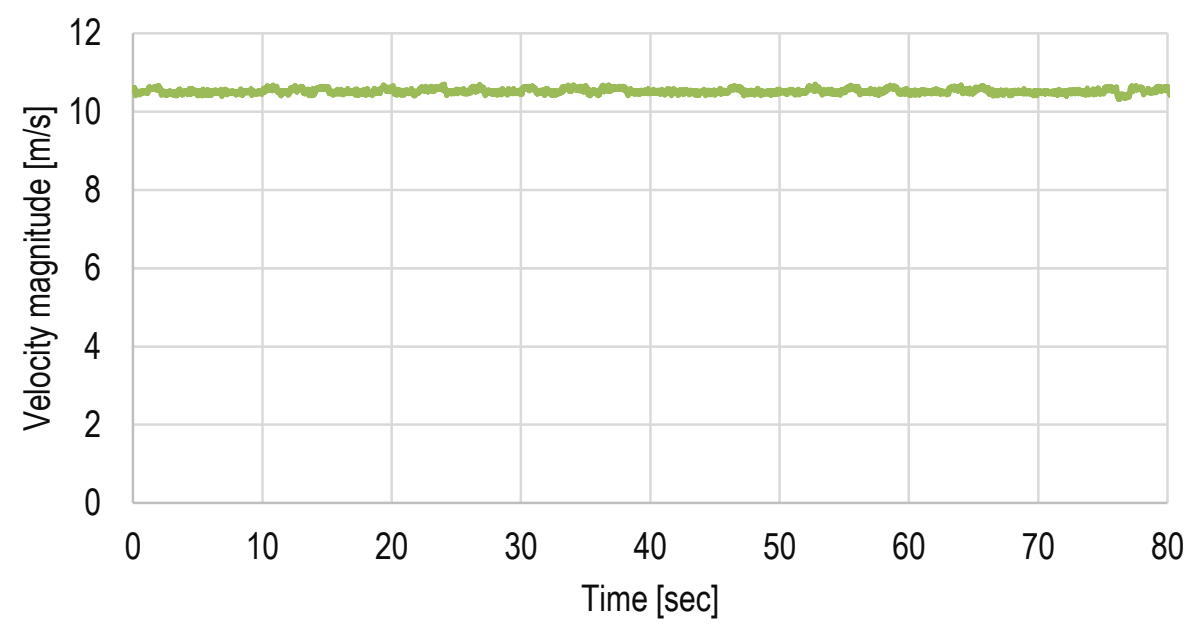

(b)

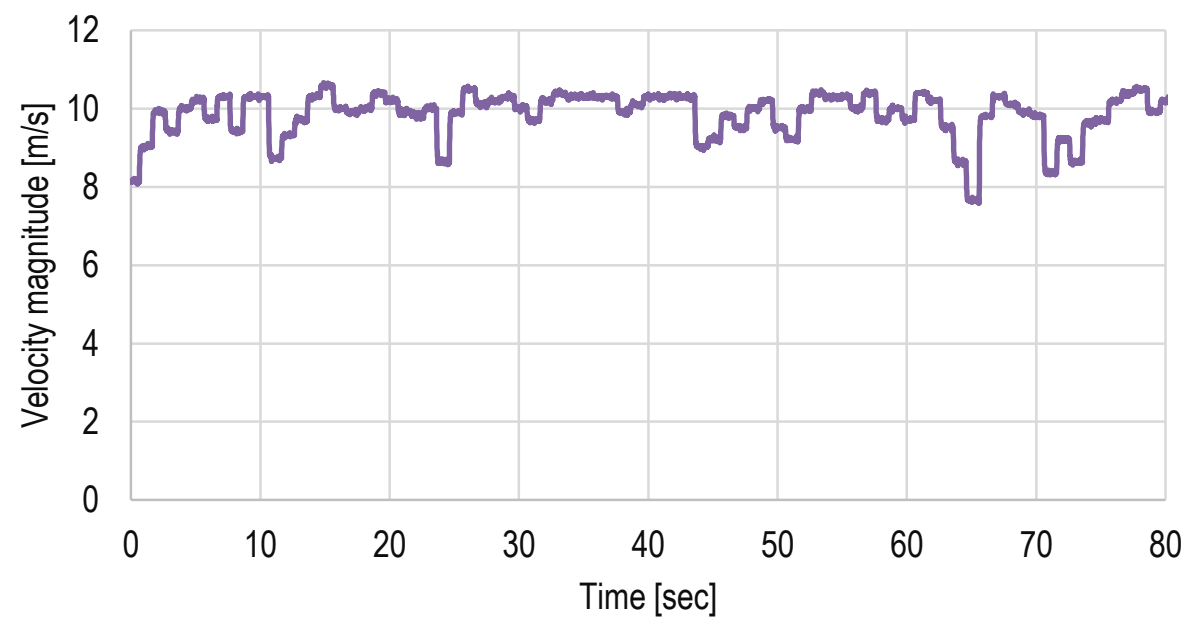

- $\mathrm{P}+23$

Figure 2-10: History of velocity magnitude (a) at point 20, (b) at point 23, at a fan speed of $437 \mathrm{rpm}$ 
(a)

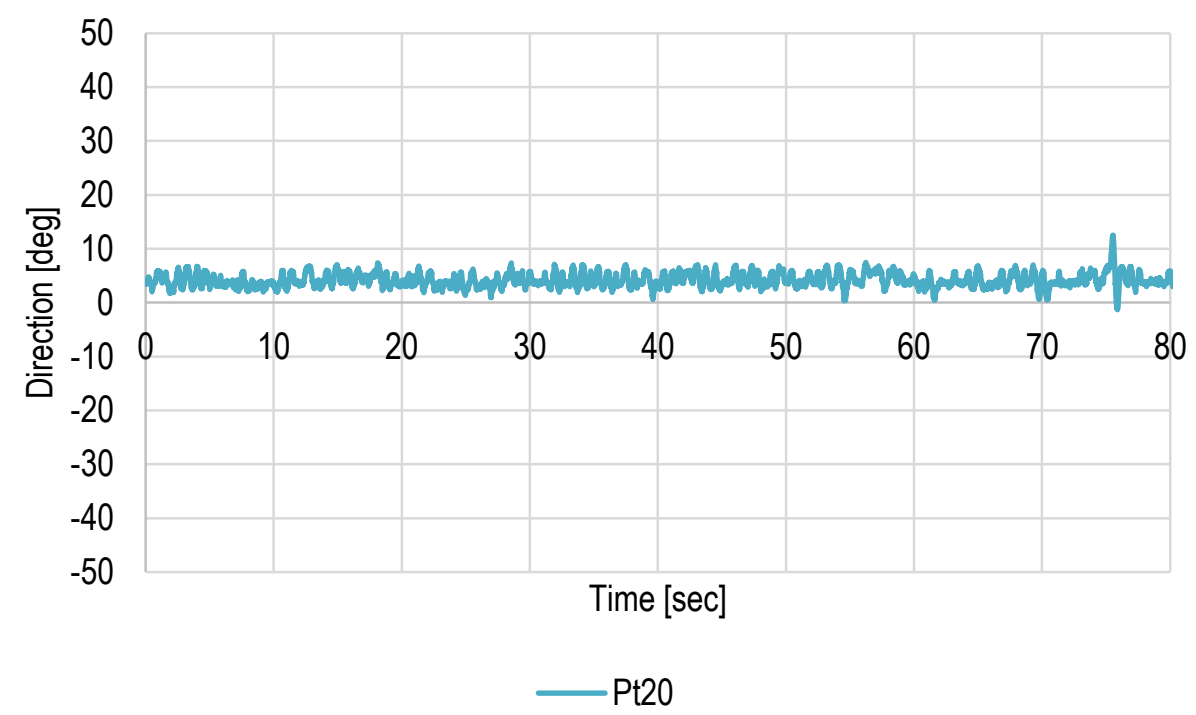

(b)

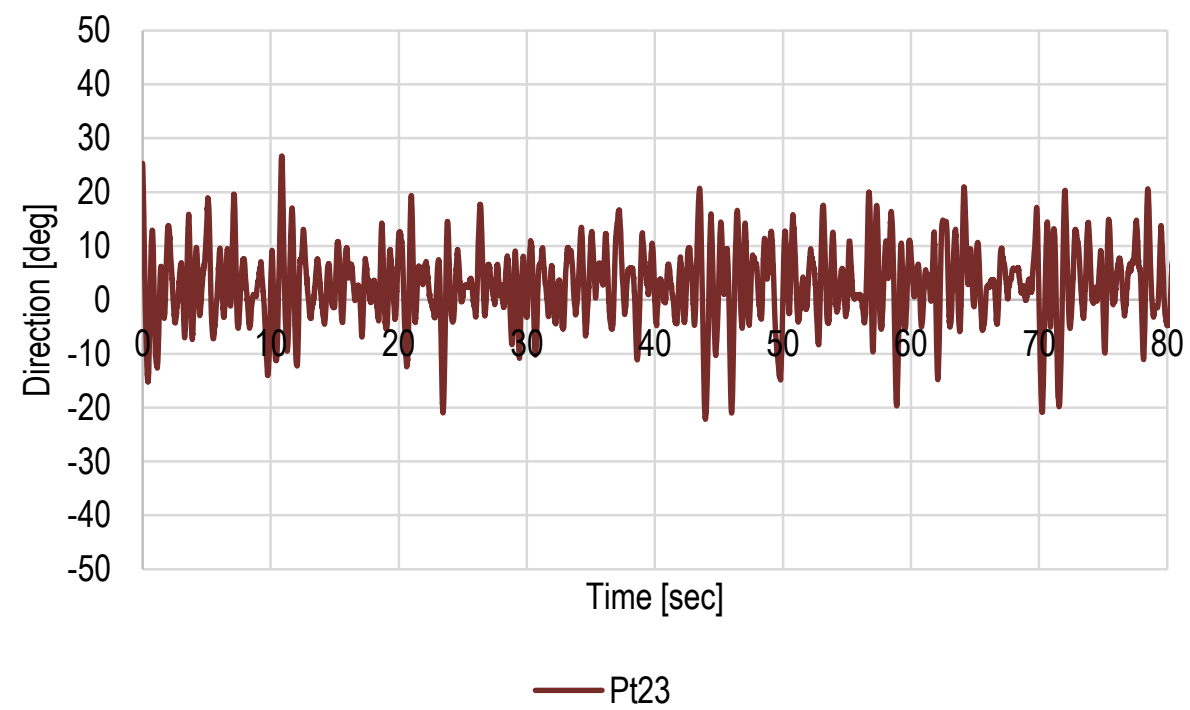

Figure 2-11: History of velocity direction (a) at point 20, (b) at point 23, at fan speed of $437 \mathrm{rpm}$

Table 2-1: Average velocity and its standard deviation at point 20

\begin{tabular}{|c|c|c|c|c|}
\hline $\begin{array}{c}\text { Fan speed } \\
\text { [rpm] }\end{array}$ & \multicolumn{2}{|c|}{219} & \multicolumn{2}{c|}{437} \\
\hline & $\begin{array}{c}\text { Velocity } \\
\text { [m/s] }\end{array}$ & Direction [deg] & $\begin{array}{c}\text { Velocity } \\
\text { [m/s] }\end{array}$ & Direction [deg] \\
\hline Average & 5.02 & 4.34 & 10.52 & 4.27 \\
\hline $\begin{array}{c}\text { Standard } \\
\text { deviation }\end{array}$ & 0.05 & 1.26 & 0.05 & 1.46 \\
\hline
\end{tabular}


Table 2-2: Average velocity and its standard deviation at point 23

\begin{tabular}{|c|c|c|c|c|}
\hline $\begin{array}{c}\text { Fan speed } \\
\text { [rpm] }\end{array}$ & \multicolumn{2}{|c|}{219} & \multicolumn{2}{c|}{437} \\
\hline & Velocity [m/s] & $\begin{array}{c}\text { Direction } \\
\text { [deg] }\end{array}$ & Velocity [m/s] & $\begin{array}{c}\text { Direction } \\
\text { [deg] }\end{array}$ \\
\hline Average & 4.64 & 2.88 & 9.83 & 2.94 \\
\hline $\begin{array}{c}\text { Standard } \\
\text { deviation }\end{array}$ & 0.33 & 8.17 & 0.54 & 7.38 \\
\hline
\end{tabular}

\section{CFD modeling}

The geometry of the model was prepared in SolidWorks [1] CAD software at TFHRC and was subsequently imported as a surface mesh into Star-CCM+ CFD software [2], where all computations were performed. Figure 3-1 (a) shows a general view of the geometry of the computational domain, with the outer wall surfaces removed to provide a clear view of structures in the room, and (b) shows a close-up of the testing section in front of the wind tunnel exit. The domain contains the wind tunnel and all major furniture and equipment located in the room. The geometry was simplified to exclude any small elements that would result in very small volume cells and generate many crevices causing the meshing and calculation process to be inefficient. Figure 3-2 presents a cross-section through the wind tunnel, to show its internal structure with screens and the fan. Initial modeling showed the importance of an accurate geometrical representation of the wind tunnel structure, especially of the fan and positions of screens. The discrepancies in the fan assembly geometry were causing significant differences in the air flow around the room between the model and measured values [3].

Several models using steady and unsteady RANS (Reynolds-Averaged Navier-Stokes) and LES (Large Eddy Simulation) solvers, different turbulence modeling and modifications in geometry were prepared [5] (see Table 3-1). The airflow was compared for these models in terms of the shape of the jet and velocity values. The wind velocity profile computed with the CFD models at the extension outlet was also compared to lab measurement [5]. Computational times are compared for runs on 64 cores, unless otherwise noted. 
(a)

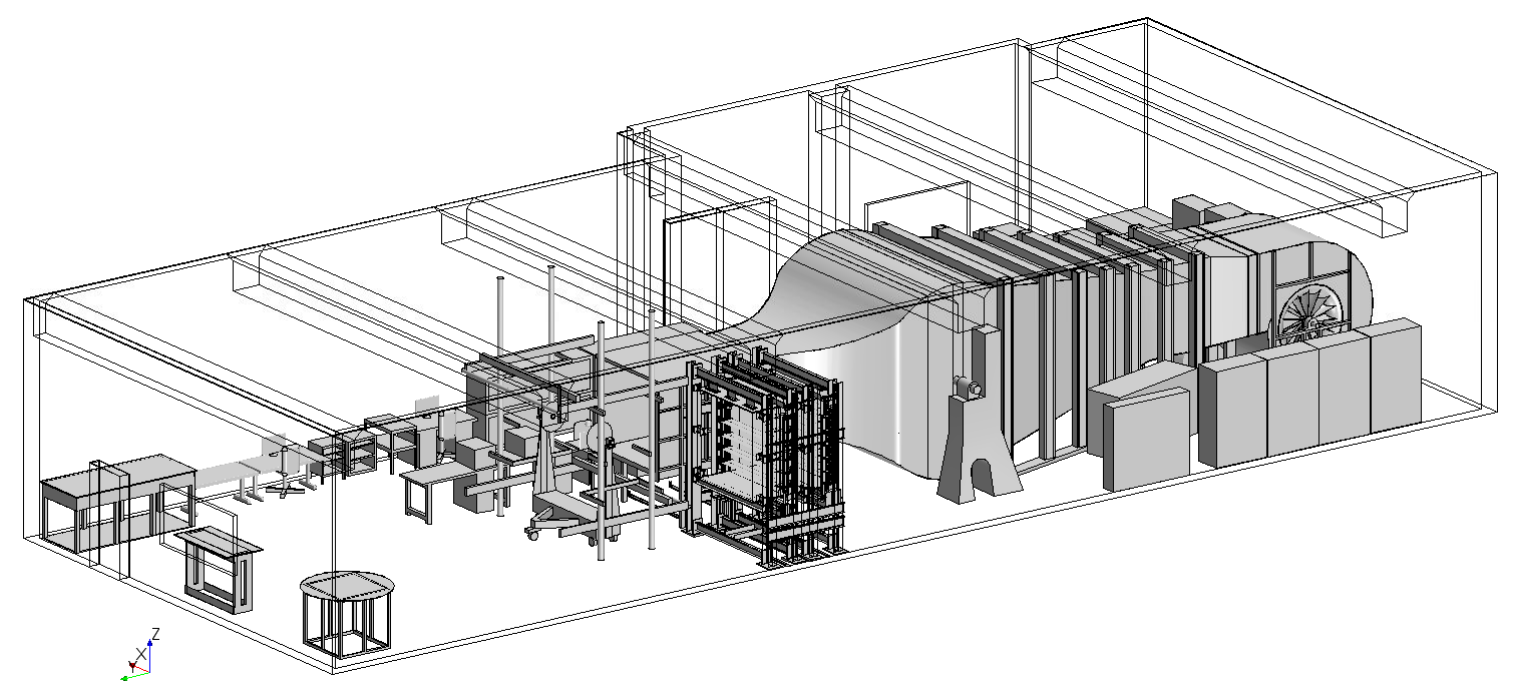

(b)

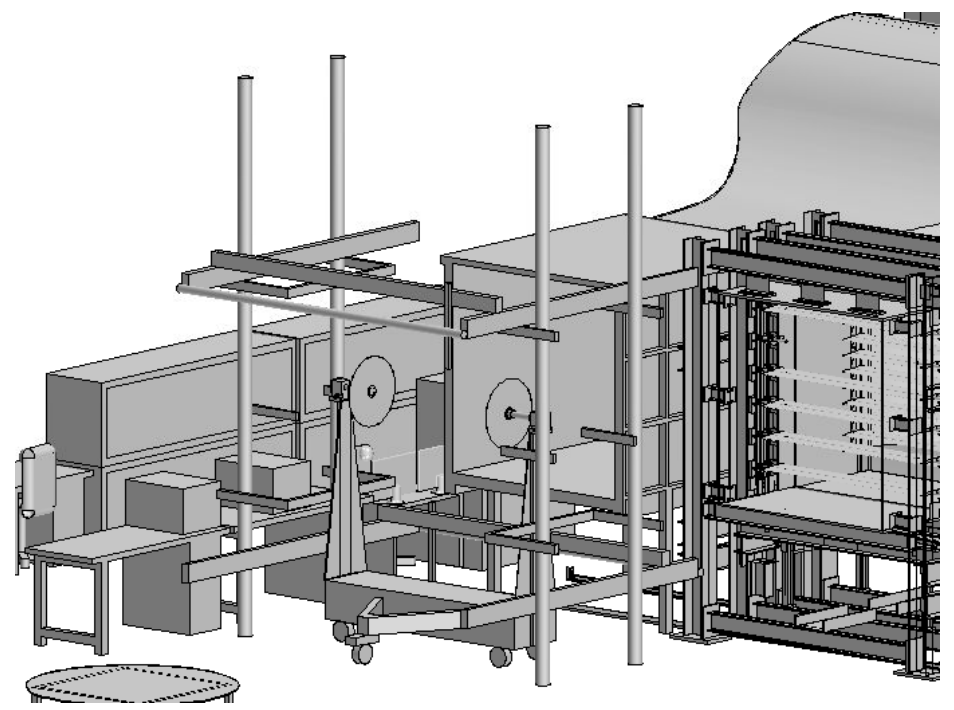

Figure 3-1: Geometry of the computational domain, (a) general view, (b) the test section in front of the wind tunnel with a turbulence generator on the right side.

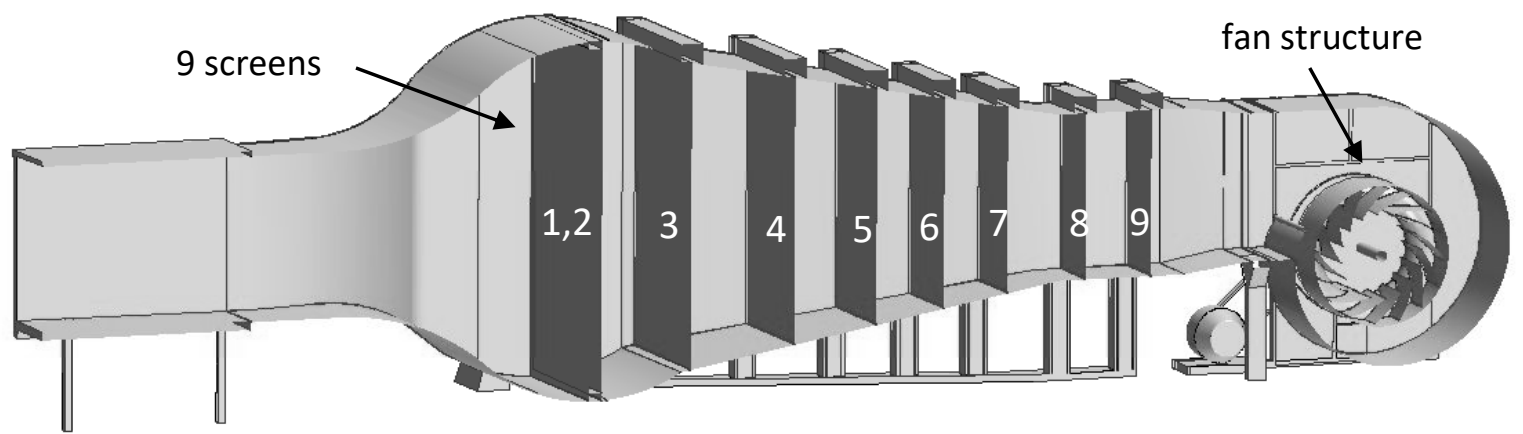

Figure 3-2: A cross section through the wind tunnel 
Table 3-1: Run times and clock times for the performed simulations

\begin{tabular}{|c|c|c|c|c|c|c|c|}
\hline $\mathbf{N r}$ & Туре & Solver & Mesh & $\begin{array}{c}\text { Number } \\
\text { of cells } \\
{\left[1^{6}\right]}\end{array}$ & $\begin{array}{l}\text { Run time } \\
\text { (4 nodes } \\
\text { unless } \\
\text { otherwise } \\
\text { noted) }\end{array}$ & $\begin{array}{c}\text { Simulated } \\
\text { Time/itera } \\
\text { tion per } \\
\text { run }\end{array}$ & $\begin{array}{c}\text { Clock } \\
\text { time } \\
\text { [days] }\end{array}$ \\
\hline 1 & $\begin{array}{l}\text { rotating } \\
\text { fan }\end{array}$ & $\begin{array}{l}\text { URANS, } \\
\quad \mathrm{k}-\varepsilon\end{array}$ & dense & 6.3 & $\begin{array}{l}1 \mathrm{sec} / 36 \mathrm{~h} \\
(8 \text { nodes })\end{array}$ & $8 \mathrm{sec}$ & 12 \\
\hline $2 a$ & \multirow{4}{*}{$\begin{array}{c}\text { rotating } \\
\text { reference } \\
\text { frame } \\
(\mathrm{RRF})\end{array}$} & \multirow{2}{*}{$\begin{array}{c}\text { RANS, } \\
\text { k- } \varepsilon\end{array}$} & coarse & 2.4 & 100oiter./2h & 10000 iter. & 0.8 \\
\hline $2 b$ & & & dense & 6.3 & 100oiter./5h & 10000 iter. & 2.1 \\
\hline $2 \mathrm{c}$ & & $\begin{array}{l}\text { URANS, } \\
\mathrm{k}-\varepsilon\end{array}$ & dense & 6.6 & $1 \mathrm{sec} / 40 \mathrm{~min}$ & $80 \mathrm{sec}$ & 2.2 \\
\hline $2 d$ & & LES & dense & $7 \cdot 7$ & $\begin{array}{l}1 \mathrm{sec} / 23 \mathrm{~h} \\
(8 \text { nodes) }\end{array}$ & $8 \mathrm{sec}$ & 7.7 \\
\hline $3 a$ & simplified & $\begin{array}{c}\text { RANS, } \\
\text { k- } \varepsilon\end{array}$ & coarse & 2.4 & $\begin{array}{c}10^{3} \text { iter. } / 40 \\
\text { min }\end{array}$ & 2000 iter & 0.06 \\
\hline $3 b$ & $\begin{array}{c}\text { inlet } \\
\text { velocity) }\end{array}$ & LES & coarse & 4.4 & $\begin{array}{c}1 \mathrm{sec}(2000 \\
\text { iter.)/52 } \\
\min \end{array}$ & $10 \mathrm{sec}$ & 0.35 \\
\hline 4 & $\begin{array}{l}\text { simplified } \\
\text { (non- } \\
\text { uniform } \\
\text { inlet } \\
\text { velocity) }\end{array}$ & $\begin{array}{c}\text { RANS, } \\
\mathrm{k}-\varepsilon\end{array}$ & coarse & 2.4 & $\begin{array}{c}10^{3} \text { iter./40 } \\
\text { min }\end{array}$ & 2000 iter & 0.06 \\
\hline 5 & $\begin{array}{l}\text { simplified } \\
\text { (half- } \\
\text { room) }\end{array}$ & $\begin{array}{c}\text { RANS, } \\
\mathrm{k}-\varepsilon\end{array}$ & coarse & 1.6 & $\begin{array}{c}10^{3} \text { iter./25 } \\
\text { min }\end{array}$ & 5000 iter & 0.09 \\
\hline
\end{tabular}




\subsection{Description of the model with a rotating fan}

Unsteady RANS (URANS) computations were performed with k- $\varepsilon$ turbulence modeling. The CFD domain was meshed with approximately 6.3 million polyhedral volume cells. It was divided into eleven regions with separate mesh parameter settings. The volumes were 'wrapped' using the Surface Wrapper technique, which facilitates the meshing process, as it usually eliminates potential problems caused by small imperfections of the geometry that are commonly present in CAD geometry files. The volumes created with the use of Surface Wrapper are: each section between screens inside of the wind tunnel (total of nine sections), the fan, and the rest of the room. A volume control was defined just downstream of the wind tunnel exit to make the mesh denser in the section used for model testing, providing a better resolution of the flow in that area. A top and a side view of plane cross sections through the room with the volume mesh are shown in Figure 3-3.

Each of the nine volumes was assigned to a region. Two types of interfaces were created on the surfaces between regions: (i) in-place internal interfaces, allowing for unobstructed flow or (ii) porous baffles, used to represent mesh screens with the flow resistance in the real wind tunnel. The walls, floor and ceiling, as well as all surfaces of the furniture were modeled as no-slip wall boundary conditions. Two internal interfaces joined the fan region with the room to allow for the return airflow from the room into the fan cage.

(a)

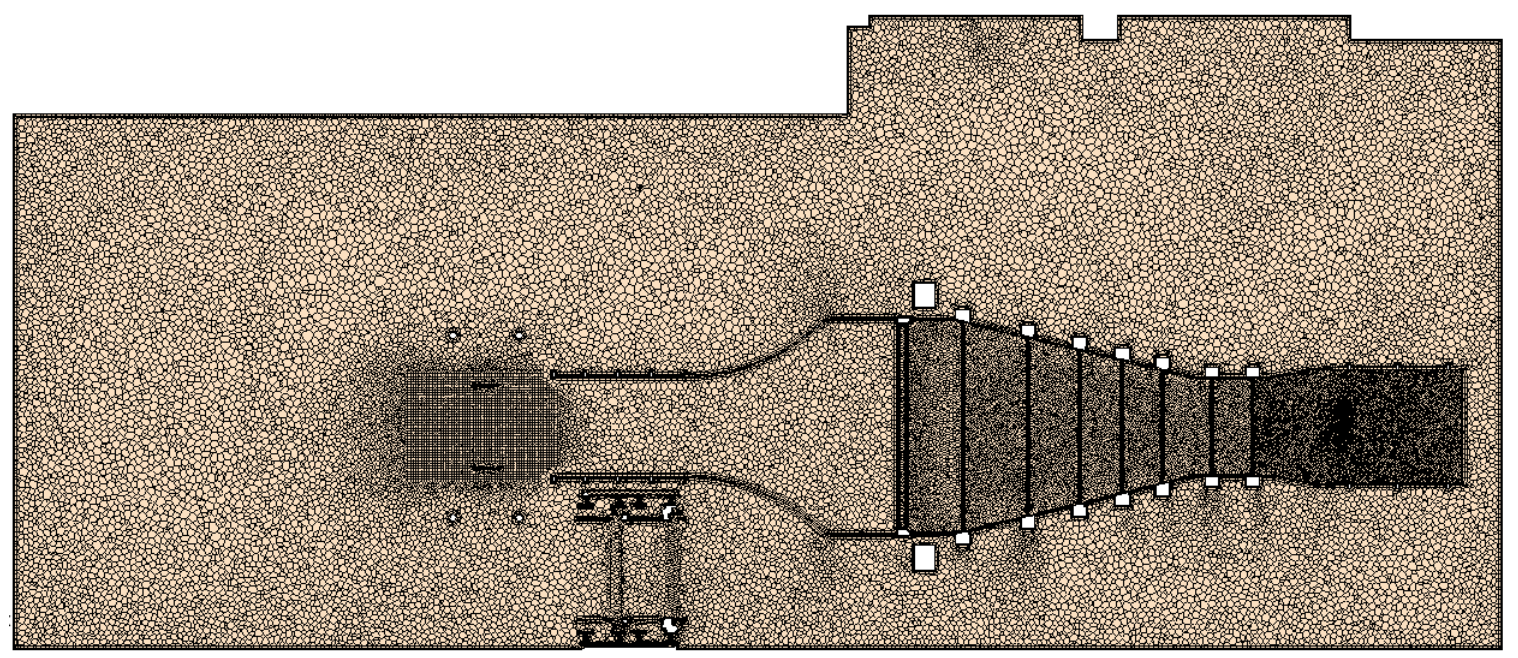

(b)

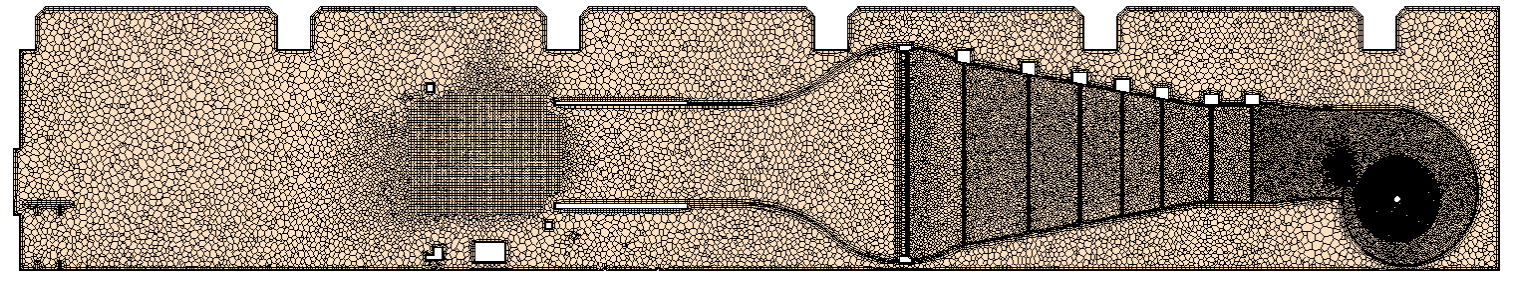

Figure 3-3: Volume mesh on the cross-section through the domain (a) top view, (b) side view 
For cases that include the rotating fan in the model domain, the fan geometry is contained in a cylinder of zero thickness surfaces defined as a separate region with an open, in-place interface. The rotation of the fan was modeled as a rigid body motion of the region around the fan axis with a specified rotational velocity. Using this approach, its rotation could be modeled without any mesh deformation. When the fan region rotates, the sliding mesh model capability is used. Flow of air can pass freely through the interface, and flow properties are interpolated in a way that preserves mass balance between the cell faces on the interfaces that are sliding past each other. The time step for the solver was set to 0.001 seconds and number of inner iterations to converge a time step was set to 40 .

Inside of the wind tunnel, there are nine screens, whose role is to make the flow more uniform in the downstream regions. They were introduced to the CFD model as porous baffle interfaces between regions. The porosity, $\chi$, of the screens was determined to be equal to 0.672 , based on the specification sheets provided by TFHRC [6].

The pressure drop across a porous baffle is modeled with the following equation [2]:

$$
\Delta p=-\rho\left(\alpha\left|v_{n}\right|+\beta\right) v_{n}
$$

with porous inertial resistance $\alpha=0.5 \cdot 6.5\left[\frac{1-\chi}{\chi^{2}}\right]\left[\frac{v_{n} d}{\chi v}\right]^{-1 / 3}$, porous viscous resistance $\beta=0$ [7], where $\rho$ is the density of air, $v_{n}$ is the normal velocity component of air flowing through the screen, the screen wire diameter is $d=0.0075$ inch and the kinematic viscosity of air is $v=15.68 \times 10^{-6} \mathrm{~m}^{2} / \mathrm{s}$.

\subsection{Description of the model with rotating reference frame (RRF)}

The computations were performed using steady and unsteady RANS solvers with a k- $\varepsilon$ turbulence model as well as with LES solver. The motion of the fan was not modeled explicitly. For a constant rigid motion, it is possible to apply a moving reference frame to the region that contains the moving part, and to solve the problem using steady-state solvers. Applying a moving reference frame to a region generates constant grid flux in the rotating domain to mimic the effects of the solid fan rotation. The moving reference frame does not change the position of cell vertices, but imposes the forces on the fluid that are induced by the rotation solid surfaces. The rotation axis direction and origin, as well as the rotation rate, are required as input to the model.

The boundary conditions in this model, as well as the screens, were the same as described in Chapter 1.

In the simulation with LES turbulence modeling, different mesh settings were used. The entire wind tunnel, including the extension section, was assigned a denser mesh. Also, the jet area in front of the wind tunnel as well as in front of the air intake for the fan was divided into smaller cells. This approach allows smaller eddies to be resolved in the flow field. To model the turbulence of eddies smaller than those that can be resolved in the mesh, the Wale subgrid scale model is used. The cross sections through a domain used for LES simulation, with volume mesh are shown in Figure 3-4. 
(a)

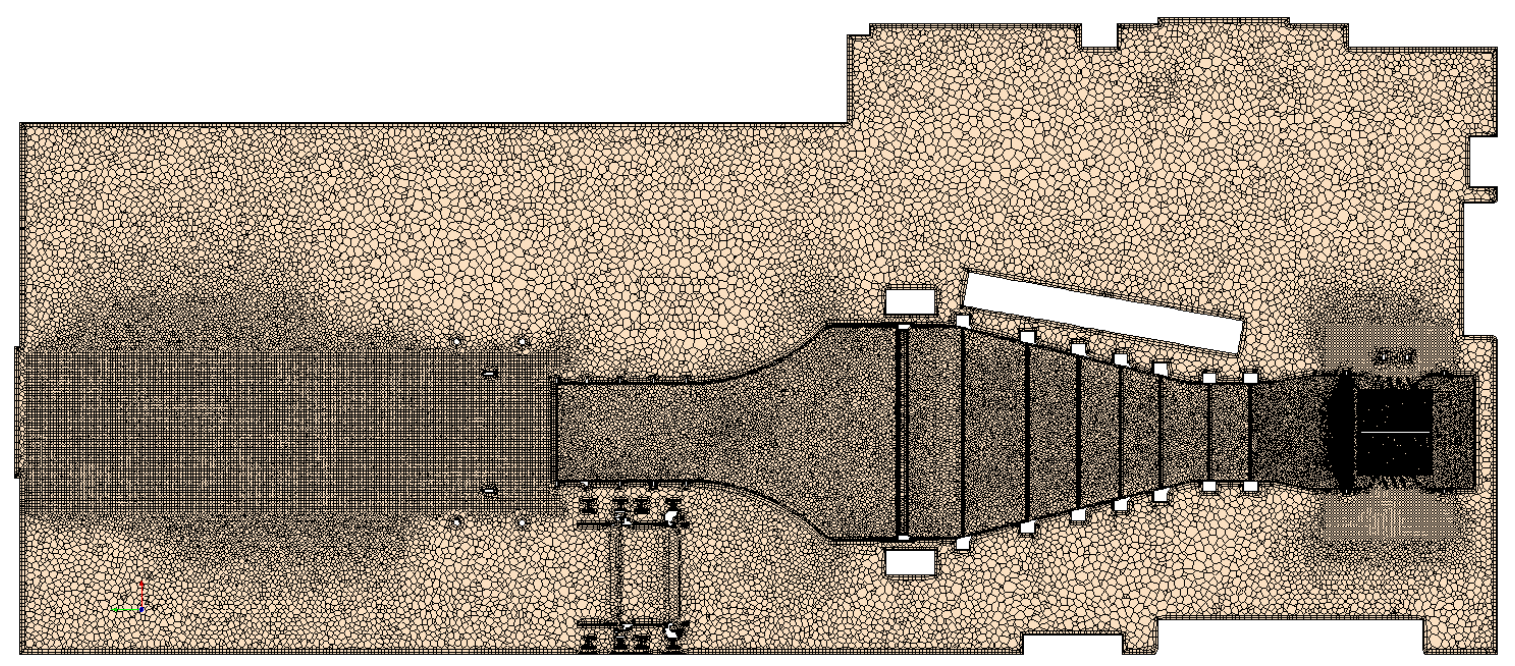

(b)

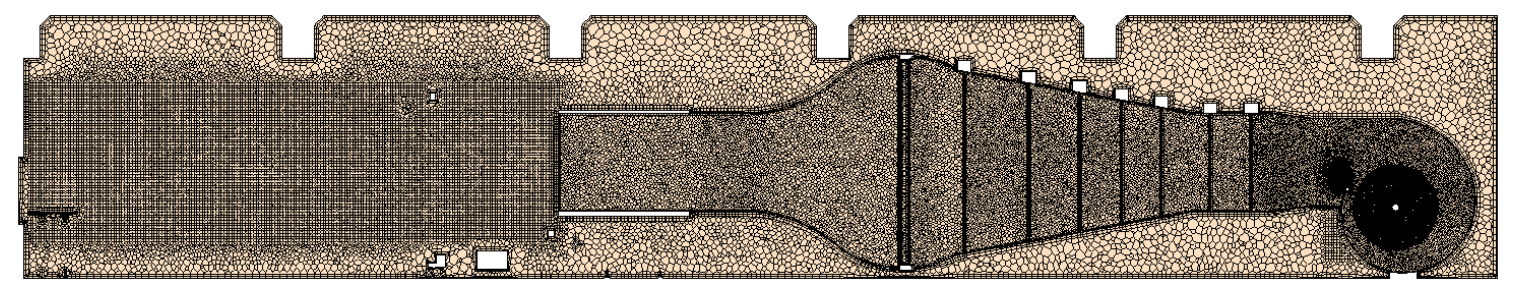

Figure 3-4: Volume mesh on the cross-section through the domain (a) top view, (b) side view, in LES simulation

\subsection{Description of the simplified models}

Simplifications of the model are proposed to reduce the time and computational resources needed to complete a run. For one of these simplifications, most of the internal structure of the wind tunnel, including the fan, was removed. The baffle (screen) closest to the tunnel exit was transformed into a velocity inlet type of boundary. Without data giving the velocity distribution at this location, the velocity at this inlet is assumed to be nearly uniform and is set to a uniform velocity normal to the inlet. The inlet velocity magnitude is obtained from a known volume flow rate, Q, of air for a specified fan motor speed and the area of the inlet, $A_{\text {in }}$. The inlet velocity magnitude is then $V_{\text {in }}=Q / A_{\text {in }}$. Assuming that the screens have functioned to make the flow entering the converging portion of the wind tunnel sufficiently uniform, a uniform velocity inlet boundary condition in a simplified model may be sufficiently accurate for engineering computations. The velocity distribution at the last screen obtained from a quasi-steady solution of the flow in the interior of the wind tunnel was also used as an inlet boundary condition for a simulation with a simplified model. The cases with uniform inlet flow and a distribution at the inlet were compared to assess the differences. In the simplified models, the two surfaces through which air enters the fan cage in the full model, are changed into pressure outlet boundary condition surfaces. A horizontal cross section of the room with marked inlet and outlets is shown in Figure 3-5. 
In the simplified models the very fine mesh in the fan cage and the fine mesh in the regions between baffles in the interior of the wind tunnel needed to accurately resolve the flow in the interior of the wind tunnel are gone because those regions are no longer part of the model domain. Removing those regions reduces the number of cells by about 60 percent. In addition, the complexity of dealing with the rotating fan via either a rotating reference frame or actually rotating the fan using sliding interfaces is also gone. These simplifications make the model much smaller and more stable, and greatly reduce the computational time necessary to reach a quasisteady converged solution. Results showing these savings will be presented in Section 4 .

An additional simplification of the model was proposed to see if additional computational resource and time savings could be obtained. The back part of the aerodynamic laboratory room was cut off and, and a new surface was created and assigned to be a pressure outlet boundary type as shown in Figure 3-6.

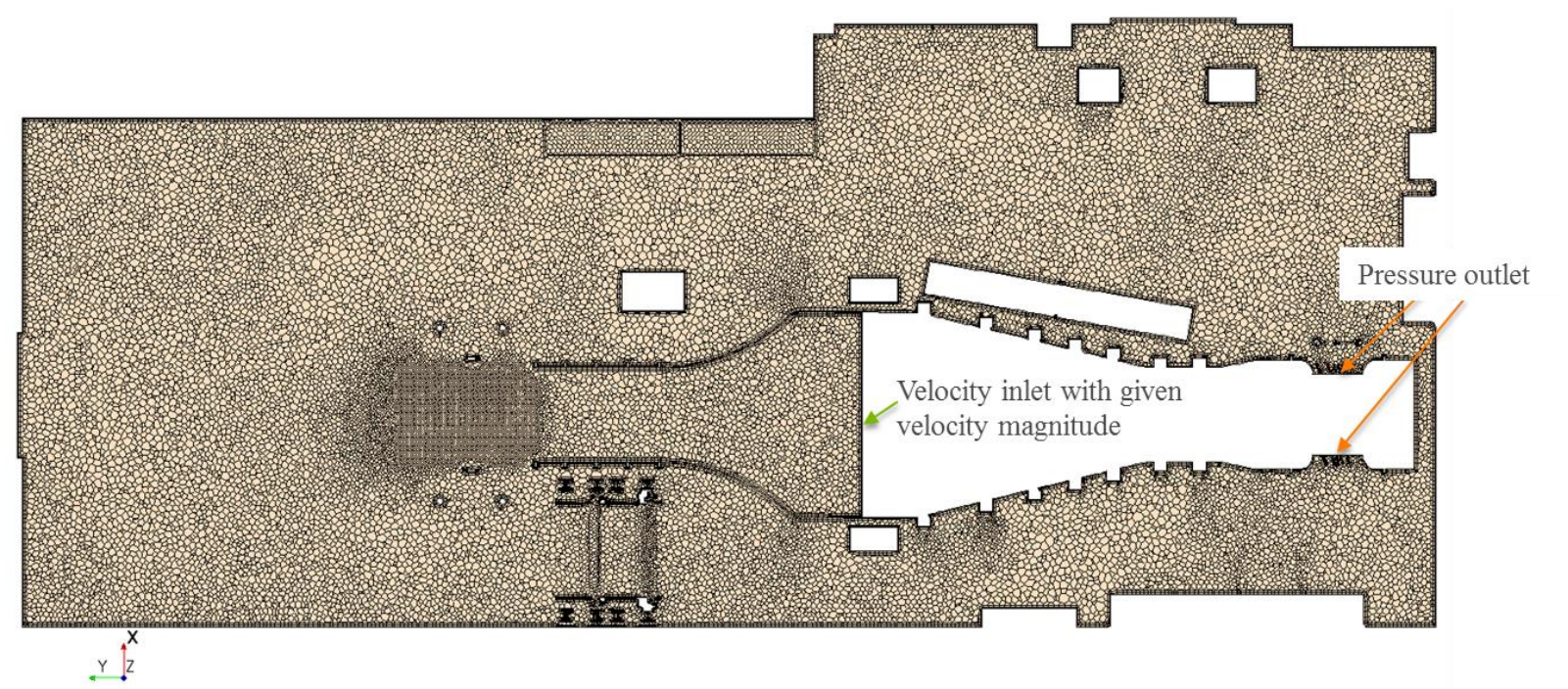

Figure 3-5: A cross-section through the simplified model

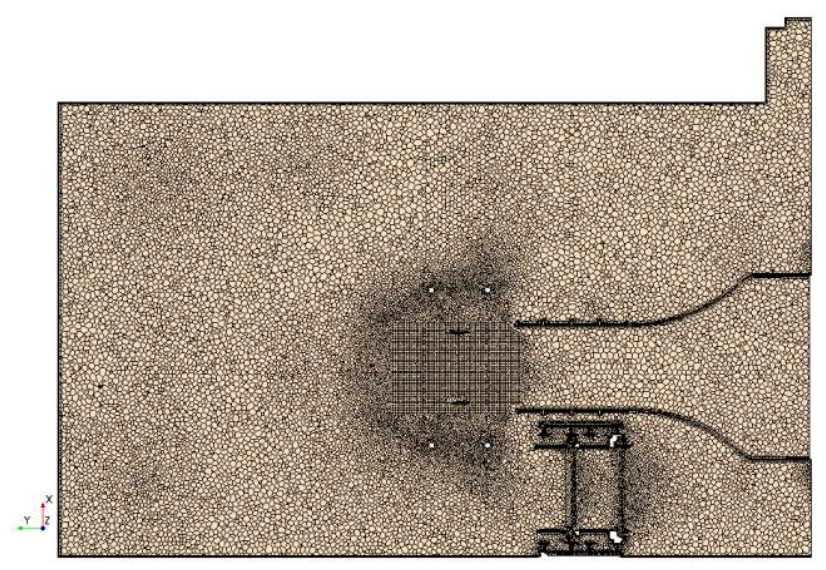

Figure 3-6: Further simplification of the model 


\section{Results and discussion}

Velocity measurements at various locations in the laboratory room, shown in Chapter 2, were used as a reference in the validation analysis of the model. Special attention was paid to points 20 and 23, which were located $2.58 \mathrm{~m}$ and $7.15 \mathrm{~m}$ downstream of the wind tunnel extension. These two points were chosen, because they were located on the center line of the wind tunnel exit, one close to the wind tunnel and the other close to a wall. The measurements showed oscillations in the readings at these locations, with bigger amplitudes of value and direction for the readings closer to the wall.

The velocity magnitude distribution was monitored on vertical and horizontal planes crossing through the center of the wind tunnel exit. The velocity component in the primary flow direction and turbulence intensity were recorded on vertical planes 1.5 inch into the wind tunnel exit, and also 1.5 inch, 1 foot, and 2 feet away from it.

\subsection{Model with rotating fan}

A full-size model of the wind tunnel laboratory with the rotating fan was created. Running this model on 64 cores took about two weeks of clock time on the TRACC Zephyr cluster. Velocities at measuring points 20 and 23 experienced high variability at the beginning of the simulation. In about 8.5 seconds of simulation time, the oscillations were reduced significantly. A plot of the $Y$ component of velocity vs. time is presented in Figure 4-1 and the final time-averaged values were combined in Table 4-1.

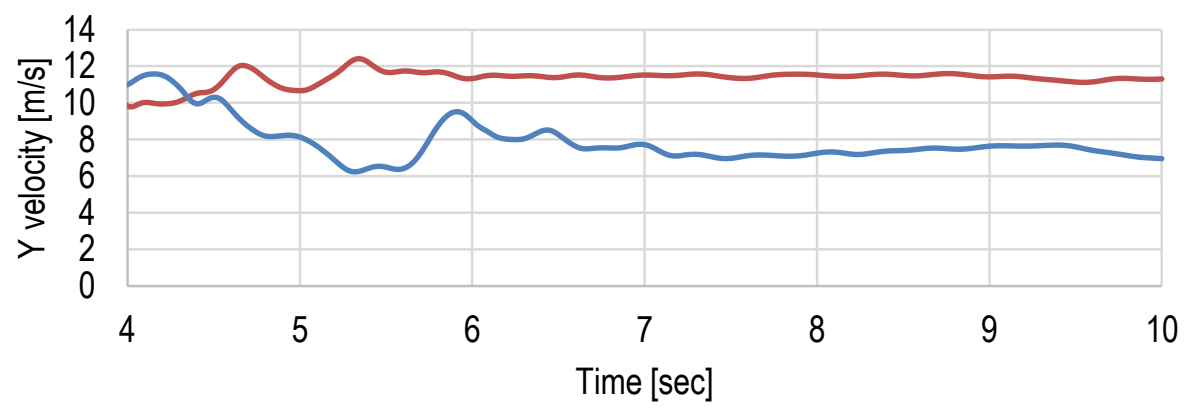

- Point $20 \longrightarrow$ Point 23

Figure 4-1: Velocity in Y direction in points 20 and 23 vs. computational time

Table 4-1: Average velocity in Y direction at points 20 and 23

\begin{tabular}{|c|c|c|}
\hline Point number & $\mathbf{2 0}$ & $\mathbf{2 3}$ \\
\hline $\begin{array}{c}\text { Average Y } \\
\text { velocity }[\mathrm{m} / \mathrm{s}]\end{array}$ & 11.50 & 7.28 \\
\hline
\end{tabular}


Figure 4-2 shows distribution plots of velocity in the $Y$ direction on planes: (a) $Y=-1.5$ in, (b) $Y=1.5$ in, (c) $\mathrm{Y}=1 \mathrm{ft}$, (d) $\mathrm{Y}=2 \mathrm{ft}$. Note that the $\mathrm{Y}$ axis direction corresponds to the direction of the center of the jet exiting the wind tunnel. An additional plot was created for the plane of point 23 in Figure 4-3, where the $Y$ velocity distribution was shown, along with an isoline of $Y$ velocity equal $9.8 \mathrm{~m} / \mathrm{s}$ (the average velocity value at point 23 from lab measurements). The distance between the location of point 23 and the center of this isoline is equal $0.58 \mathrm{~m}$. This shows that the difference between the experimental and computational results is small, and that the difference between experimental measurements and model results in wind speed at point 23 near the stagnation wall are primarily due to the position of the jet as it turns for the return flow. In RANS simulations, the bulk of the jet tends for shift a bit toward the more open side of the room (front door side) and that pattern persists because RANS uses steady state solvers.

(a) $Y=-1.5$ in

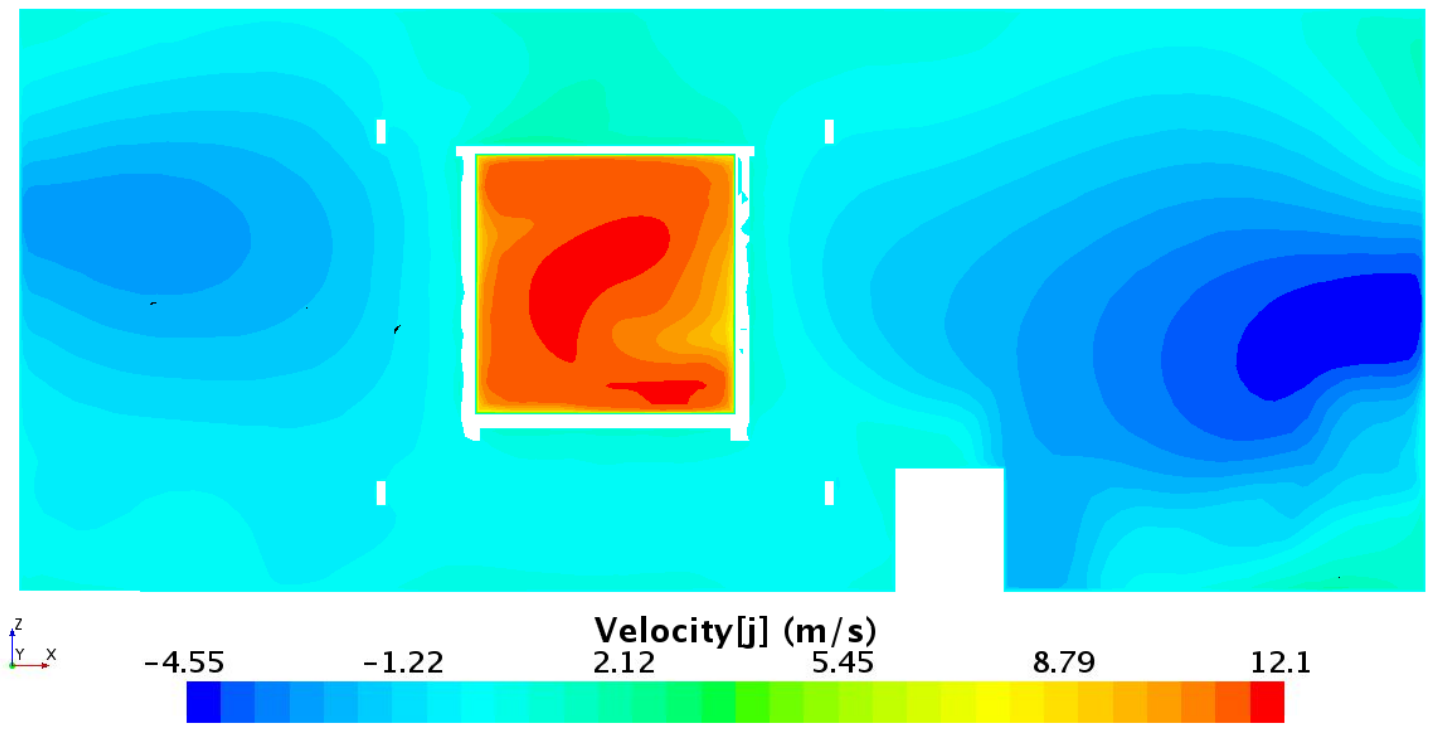

(b) $Y=1.5$ in

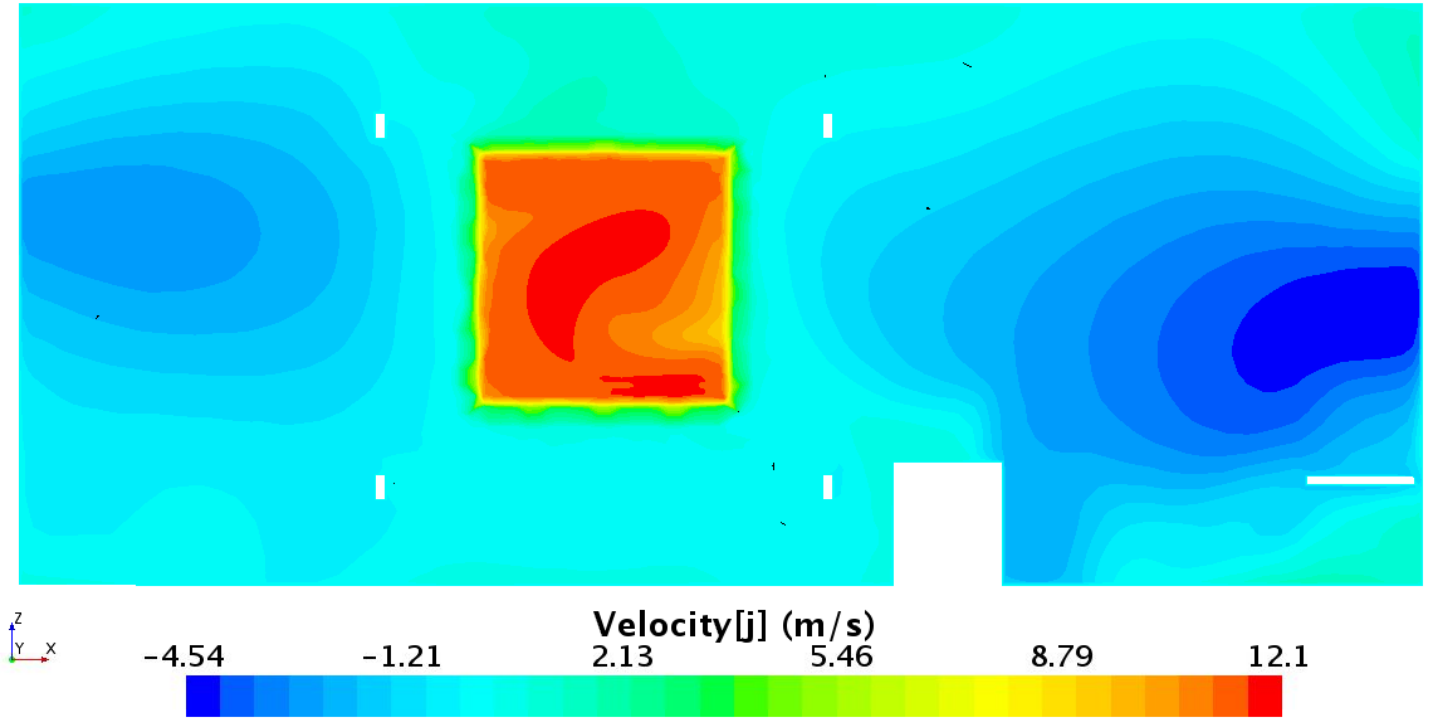


(c) $\mathrm{Y}=1 \mathrm{ft}$
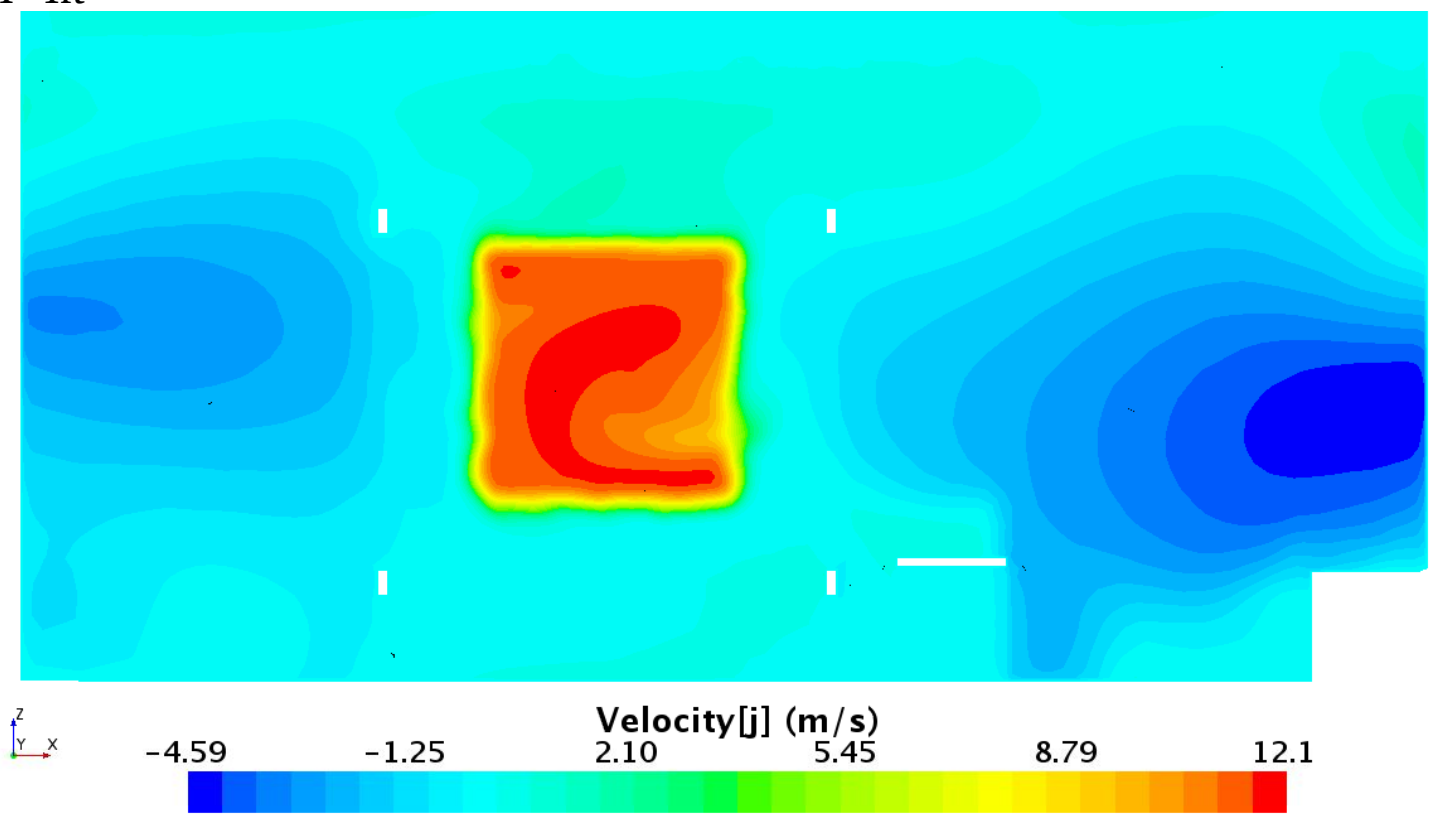

(d) $\quad \mathrm{Y}=2 \mathrm{ft}$
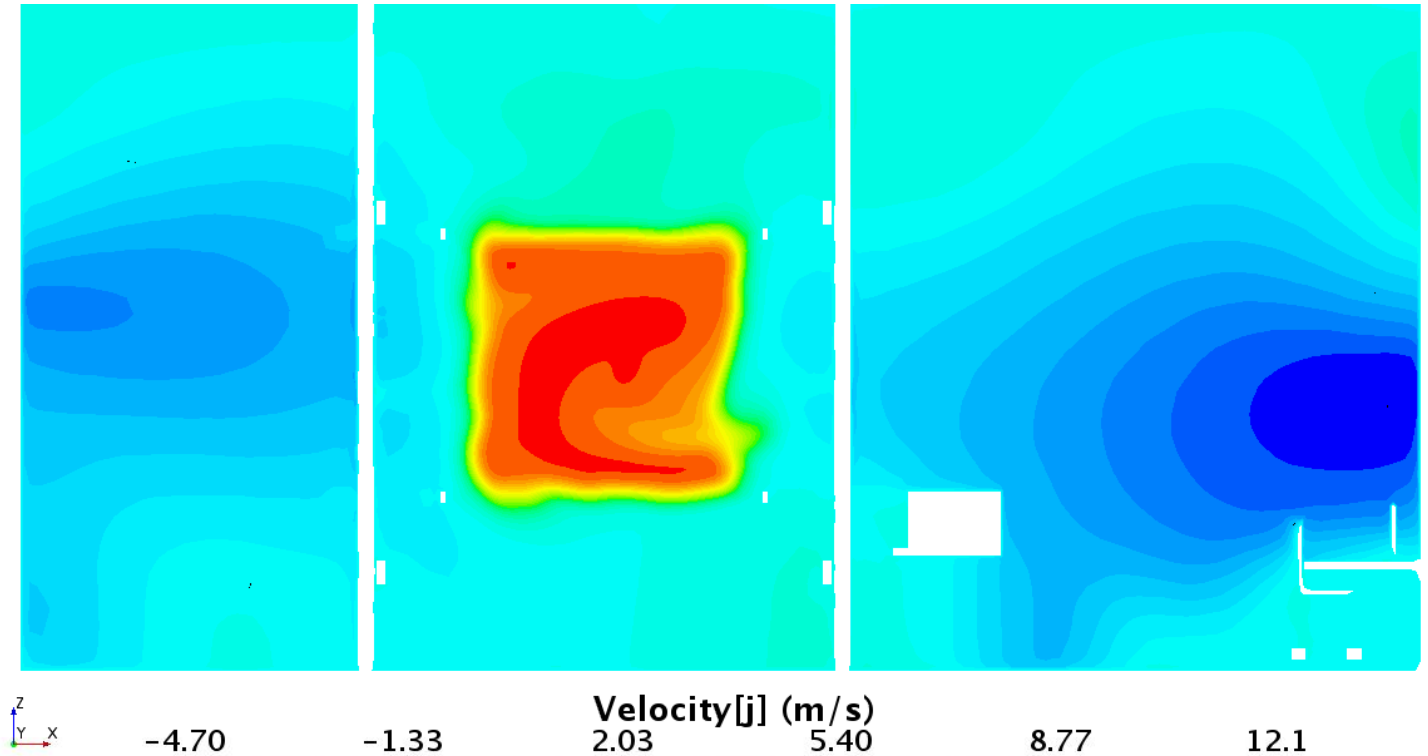

$\underset{2.03}{\operatorname{Velocity}[\mathrm{j}]} \underset{5.40}{(\mathrm{~m} / \mathrm{s})}$

8.77

12.1

Figure 4-2: Distribution of velocity in $Y$ direction on planes: (a) $Y=-1.5$ in, (b) $Y=1.5$ in, (c) $Y=1 \mathrm{ft}$, (d) $Y=2$ $\mathrm{ft}$. Model with rotating fan. 


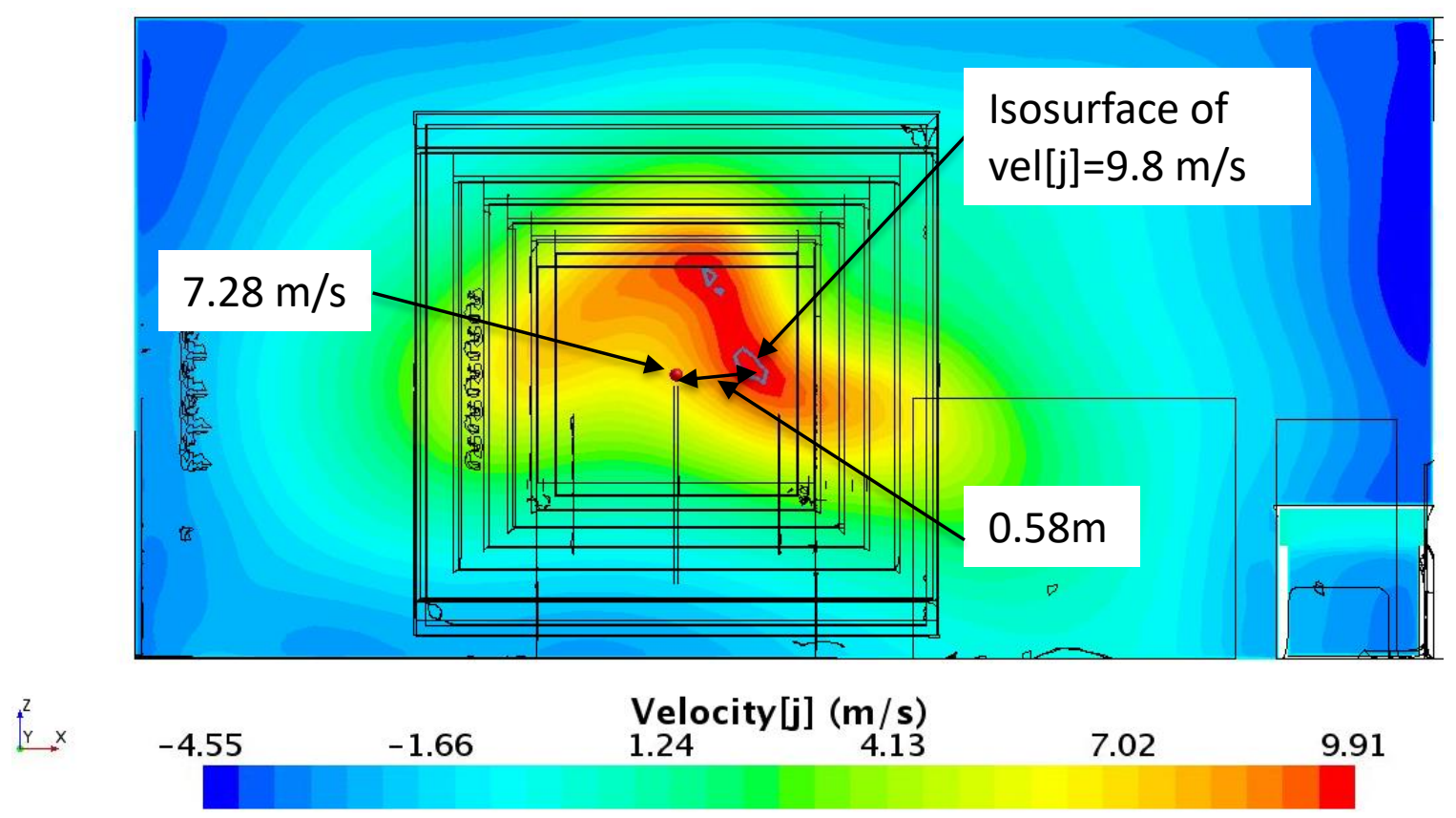

Figure 4-3: Distribution of velocity in Y direction on a vertical plane of point 23. Model with rotating fan.

\subsection{Model with rotating reference frame}

As described in Section 3.2, there are no moving meshes in the rotating reference frame (RRF) model. For computational efficiency, it imparts forces to the air moving through the fan region that would result from the rotating fan surfaces at the user specified rotation rate.

\subsubsection{RANS, k- $\varepsilon$ turbulence modeling}

A run using the RRF model with the RANS, $\mathrm{k}-\varepsilon$ turbulence model takes about 24 hours on four computational nodes (64 cores) to complete. The simulation had to run for about ten thousand iterations to achieve a converged and stable solution throughout the domain. Some of the final velocities registered across the room fluctuated from iteration to iteration and averaging was applied to the results. The fluctuations are attributed to the fact that the flow recirculates in a closed domain and feedback can occur between flow in wind tunnel and flow in the room. Near walls, significant fluctuations were also observed in some of the anemometers in the experiments run in the aerodynamics laboratory. 
(a)

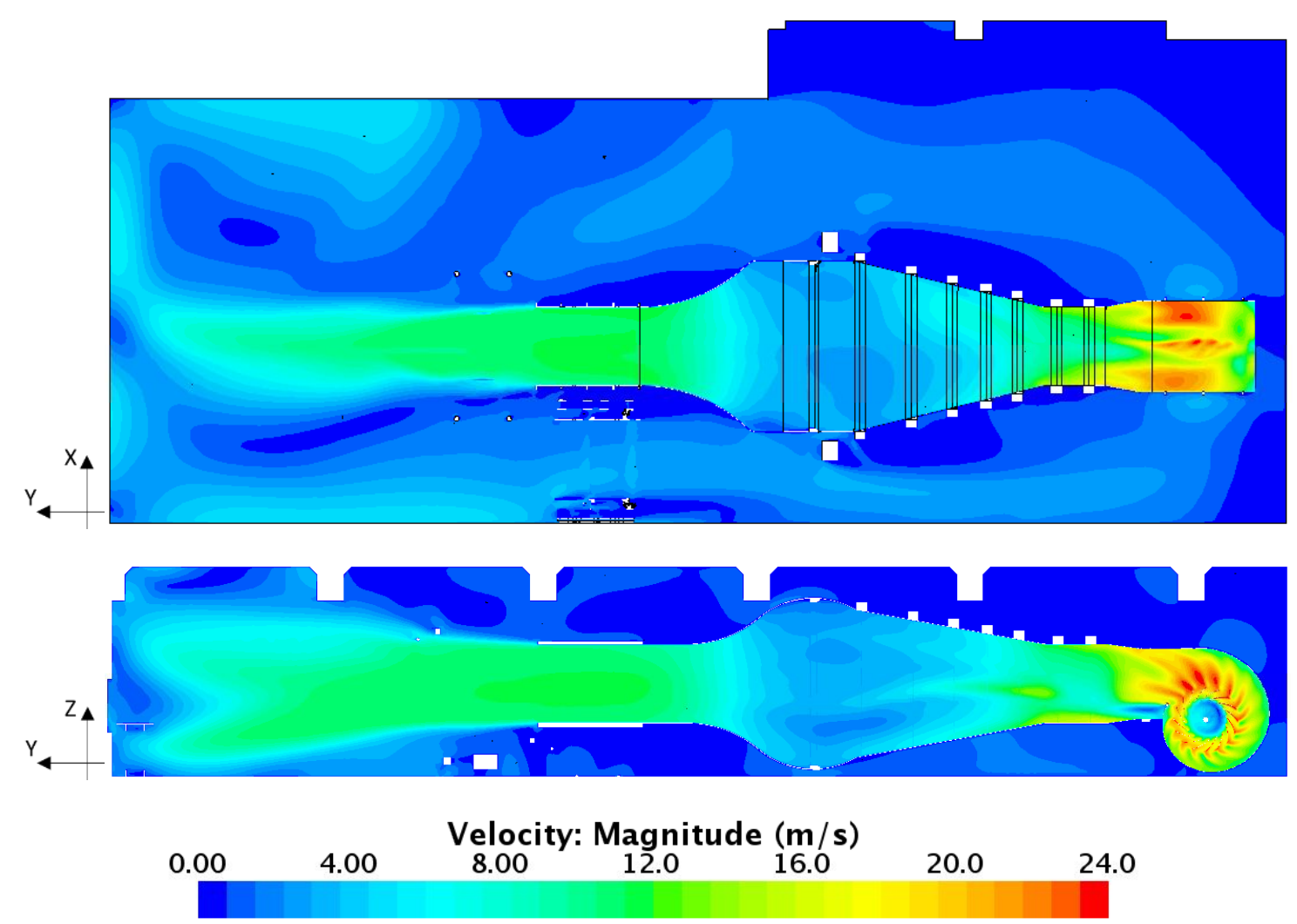

Figure 4-4: Velocity magnitude on (a) horizontal and (b) vertical planes. Model with rotating reference frame, RANS solver.

A comparison of pressure drop across screens for two formulations of parameter $\alpha$, the porous inertial resistance of a screen, was performed and the results were shown in Table 4-2. One of the formulations was based on averaged velocity over the area of a screen, the second on the local velocity distribution. The surface averaged velocities were the same in both cases. The pressure drop did not change much. The highest difference, a drop by $4 \%$, was recorded on screen number 1 , which was the one closest to the fan. The difference on screen number 9 , the furthest from the fan, equaled $0.5 \%$. The distribution of (a) normal velocity and (b) inertial porous resistance of the screens for $\alpha$ depending on local velocity values is illustrated in Figure 4-5. 
Table 4-2: Pressure drop across screens for two different formulations of porous inertial resistance.

Numbering of the screens in Figure 3-2. Model with rotating reference frame, RANS solver.

\begin{tabular}{|c|c|c|c|c|c|c|c|c|c|c|}
\hline \multicolumn{2}{|c|}{ Screen number, i } & 1 & $\mathbf{2}$ & $\mathbf{3}$ & $\mathbf{4}$ & $\mathbf{5}$ & $\mathbf{6}$ & 7 & $\mathbf{8}$ & $\mathbf{9}$ \\
\hline \multicolumn{2}{|c|}{ Averaged velocity } & 10.720 & 10.719 & 8.255 & 6.247 & 4.820 & 3.665 & 2.714 & 2.484 & 2.473 \\
\hline \multicolumn{2}{|c|}{ Alpha [i] } & 0.409 & 0.409 & 0.447 & 0.490 & 0.534 & 0.586 & 0.647 & 0.667 & 0.668 \\
\hline $\begin{array}{c}\text { Pressure } \\
\text { drop }\end{array}$ & vel[j] $]_{\text {ave }}$ & 60.513 & 56.077 & 35.995 & 22.716 & 14.820 & 9.479 & 5.717 & 4.911 & 4.842 \\
\cline { 2 - 11 } & vel[j] & 58.165 & 56.278 & 36.243 & 22.882 & 14.96 & 9.539 & 5.770 & 4.939 & 4.866 \\
\hline
\end{tabular}

(a)

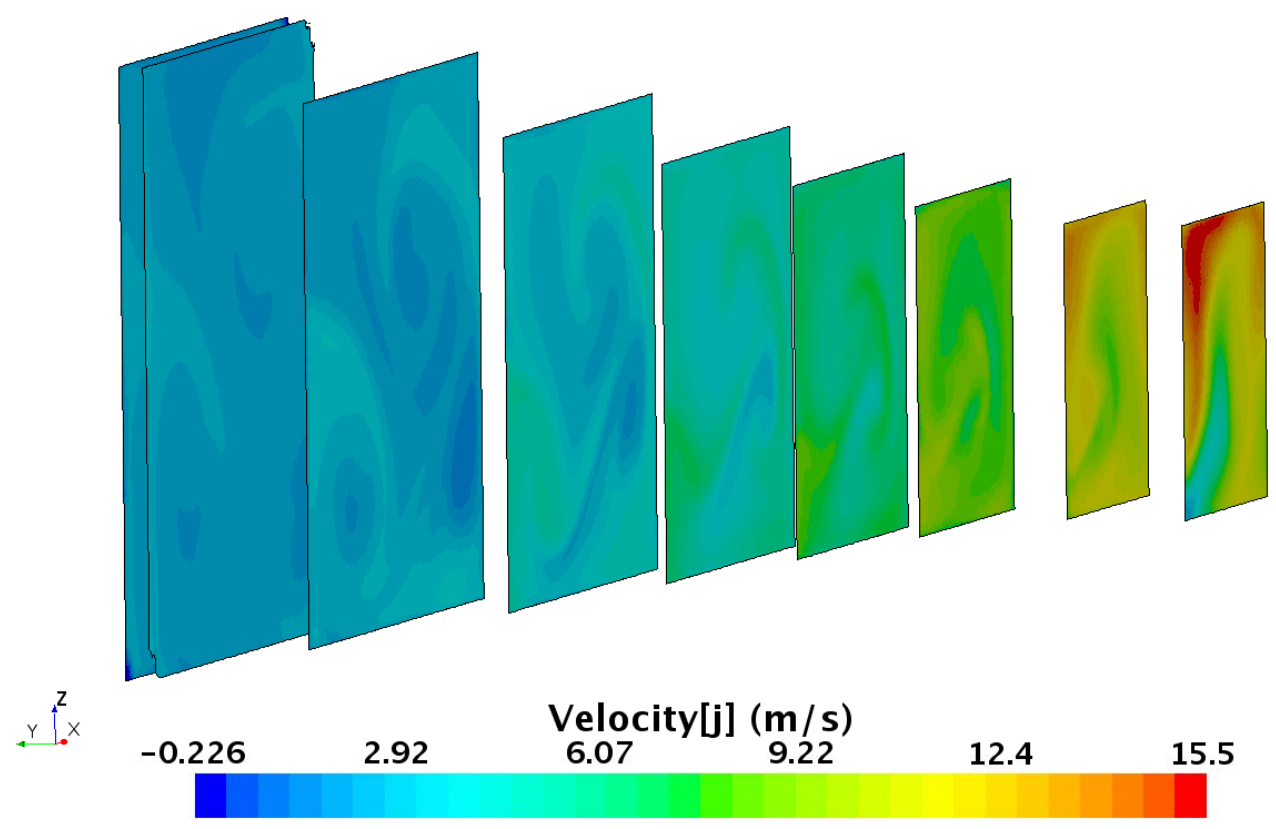


(b)

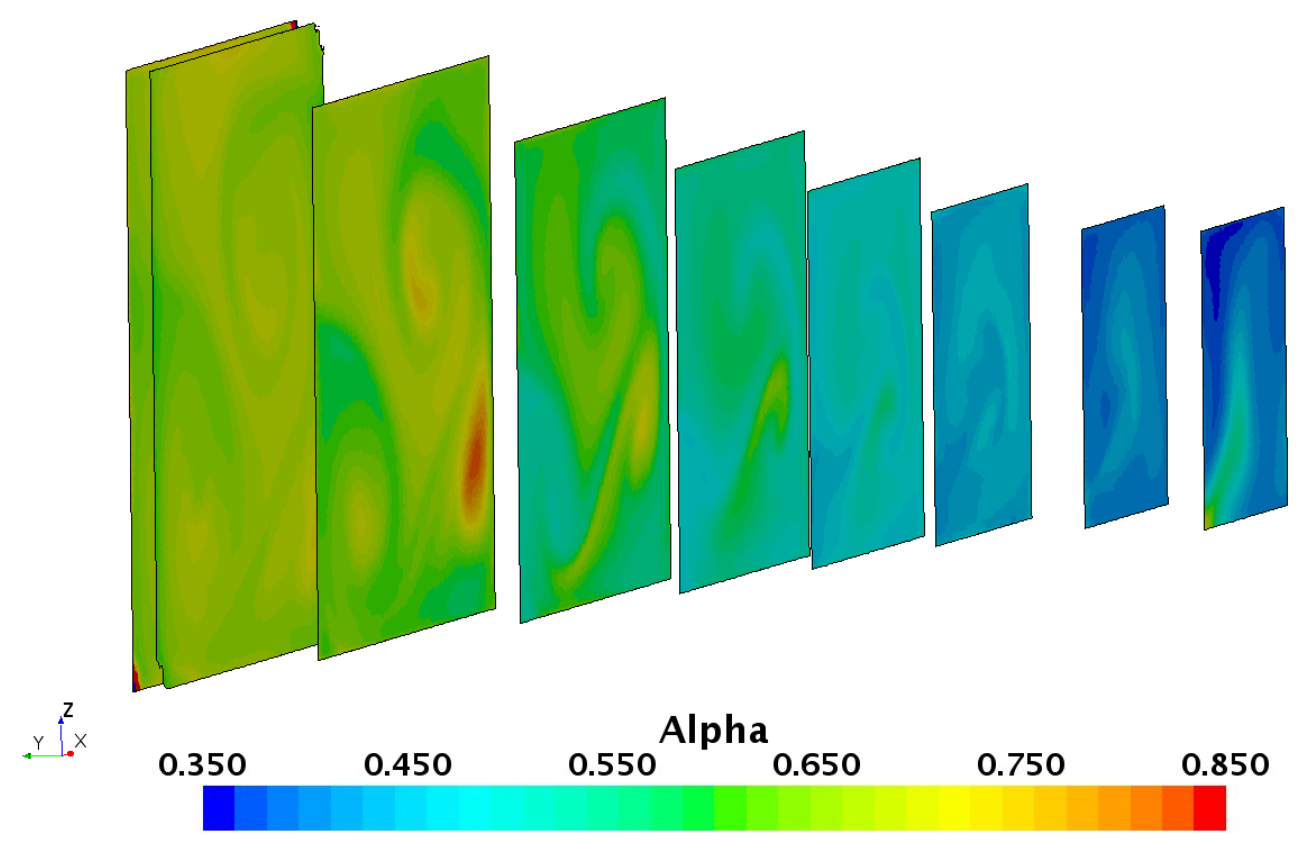

Figure 4-5: Distribution of (a) normal velocity, (b) inertial porous resistance of the screens for a depending on local velocity values. Model with rotating reference frame, RANS solver.

Mass flow through the wind tunnel was balanced and constant in this model and it had the same value as in the rotating fan simulation. The velocities in the jet varied during the simulation, but the average velocities at the screens at the end of the simulation were constant and equal to the volume flow rate in the tunnel divided by screen area. The average velocity at point 20 was equal to $11.1 \mathrm{~m} / \mathrm{s}$ and at point 23 it was equal to $7.6 \mathrm{~m} / \mathrm{s}$. Oscillations of these velocities with iteration is shown in Figure 4-6. The further from the outlet, the higher were the amplitudes of oscillation. This model gave a solution in about 10000 iterations, computed for 24 hours on 4 nodes, each with 16 floating point cores. 


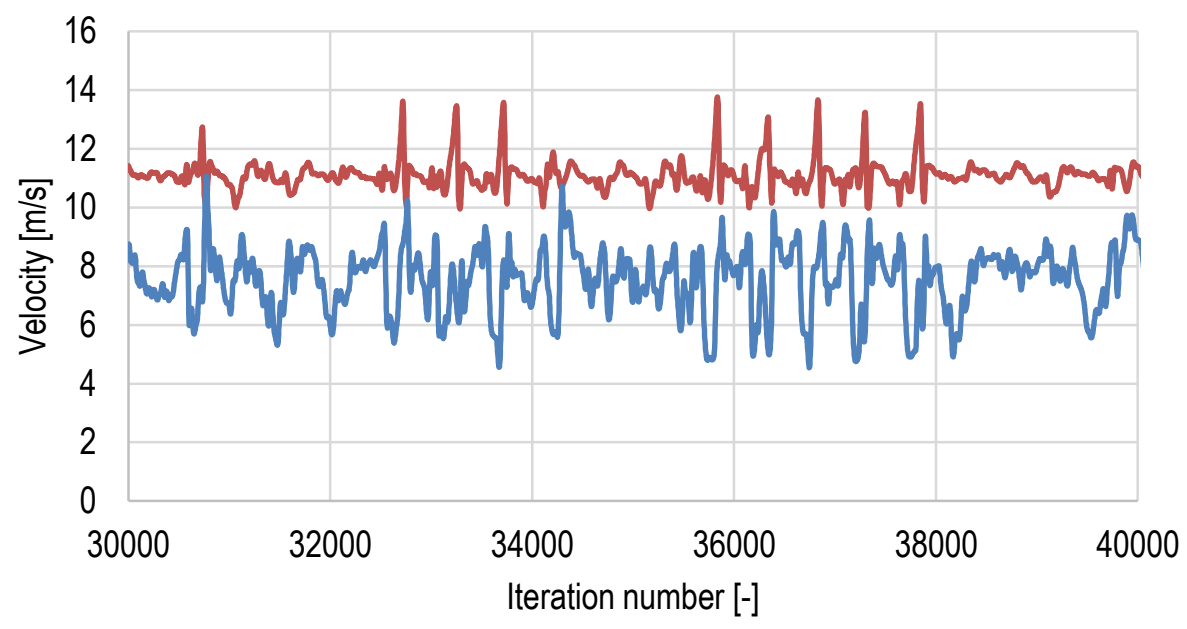

—Point 20 —Point 23

Figure 4-6: Velocity magnitude at points 20 and 23. Model with rotating reference frame, RANS solver.

Figure 4-7 shows the distribution of velocity in $\mathrm{Y}$ direction on planes: (a) $\mathrm{Y}=-1.5$ in, (b) $\mathrm{Y}=1.5$ in, (c) $\mathrm{Y}=1 \mathrm{ft}$, and (d) $\mathrm{Y}=2 \mathrm{ft}$ and Figure 4-8 shows the turbulence intensity distribution on those planes.

(a) $Y=-1.5 \mathrm{in}$

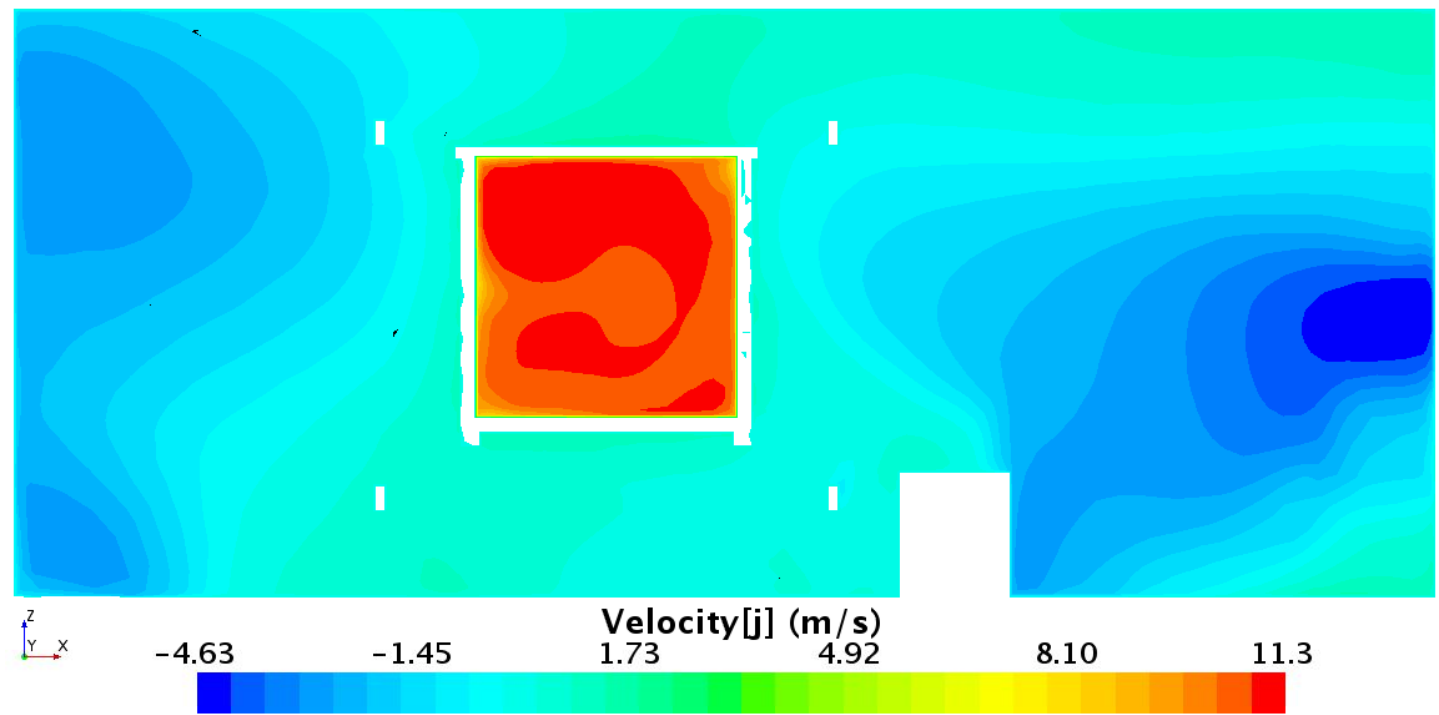


(b) $Y=1.5$ in

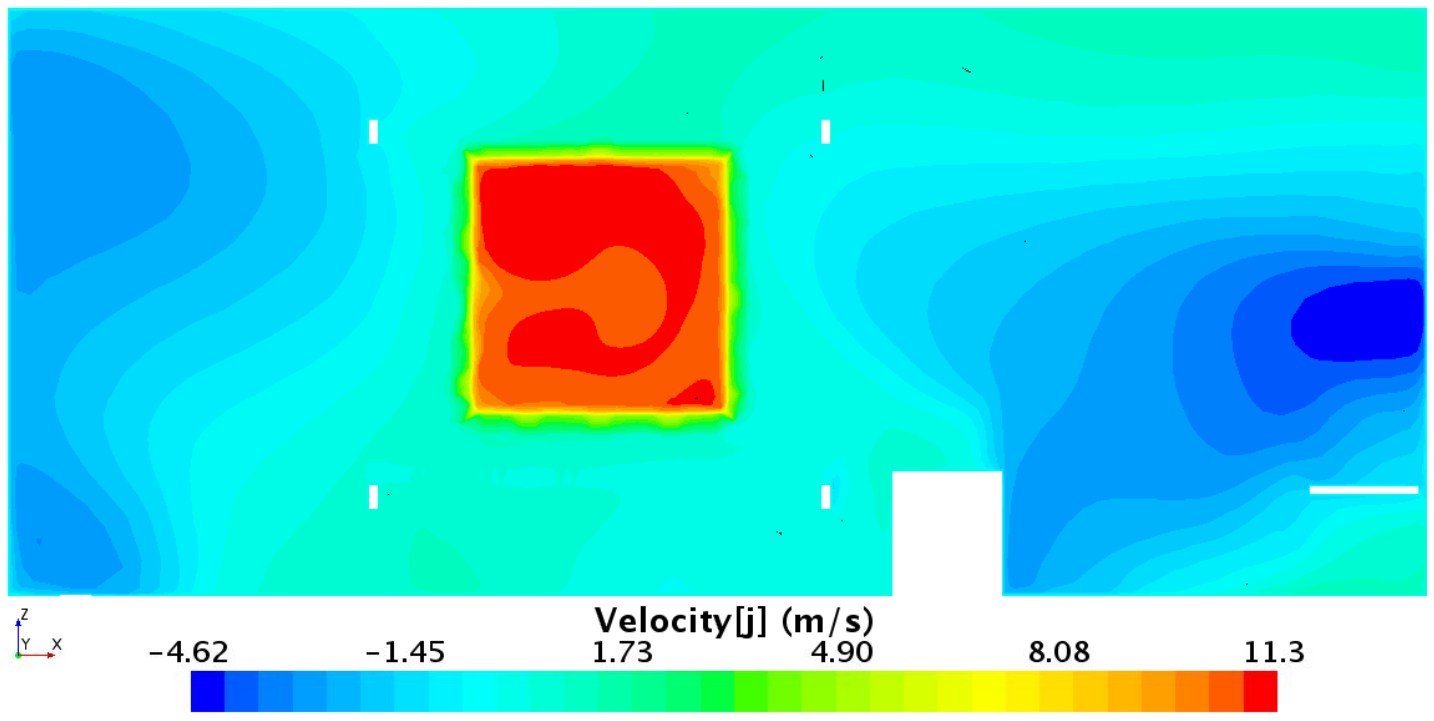

(c) $\quad \mathrm{Y}=1 \mathrm{ft}$

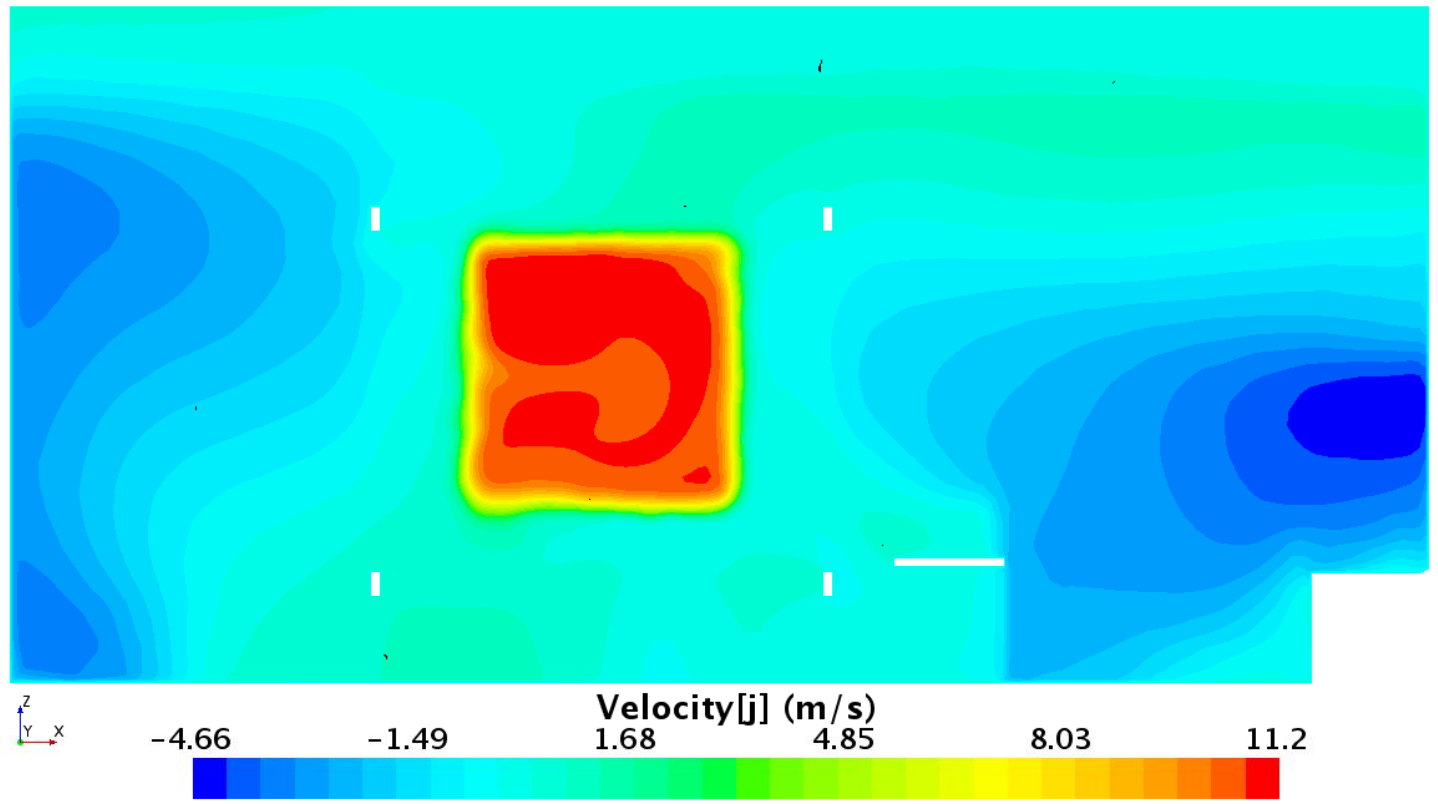


(d) $\quad \mathrm{Y}=2 \mathrm{ft}$

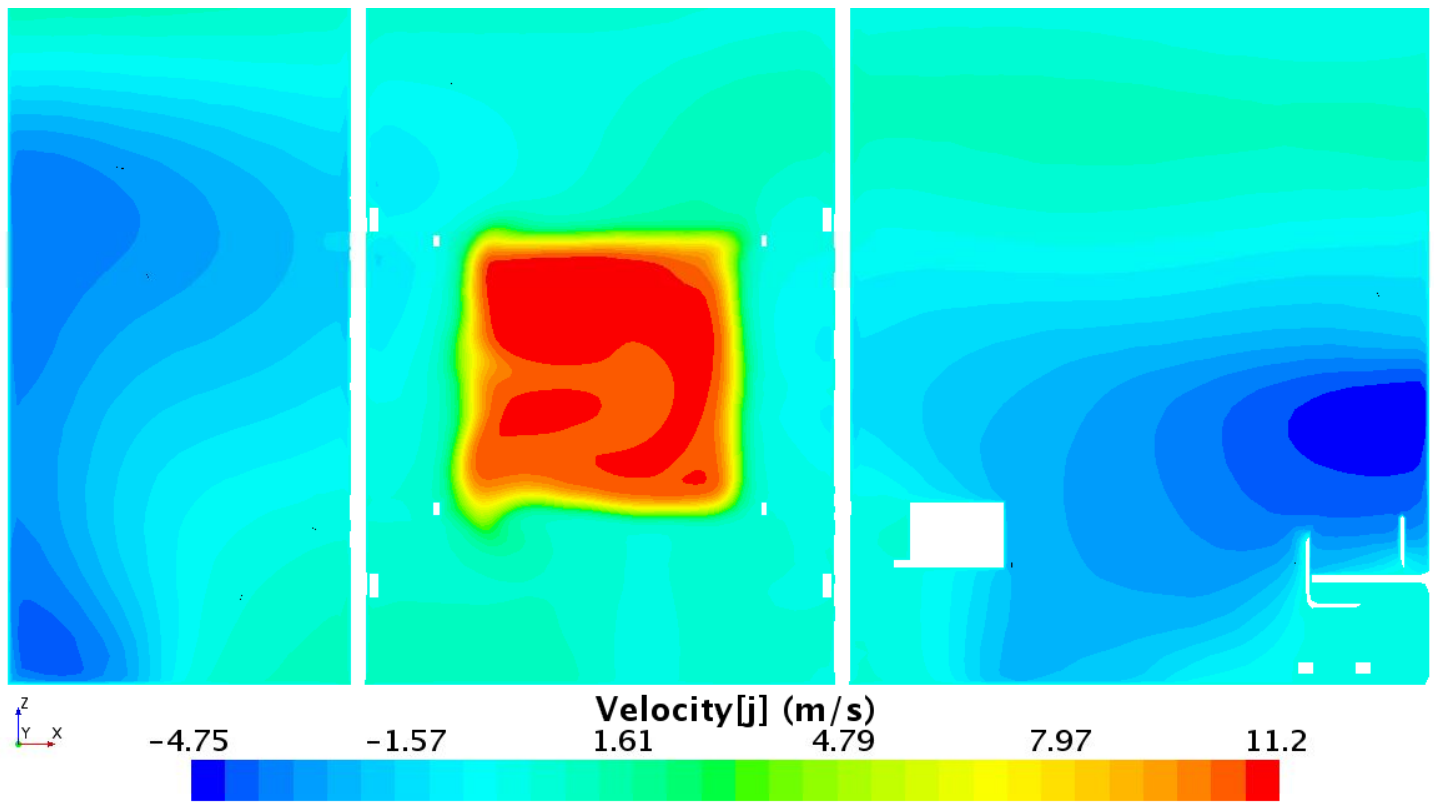

Figure 4-7: The distribution of velocity in $\mathrm{Y}$ direction on vertical planes: (a) $\mathrm{Y}=-1.5$ in, (b) $\mathrm{Y}=1.5$ in, (c) $\mathrm{Y}=1$ $\mathrm{ft}$, (d) $\mathrm{Y}=2 \mathrm{ft}$. Model with rotating reference frame, RANS solver.

(a) $\mathrm{Y}=-1.5 \mathrm{in}$

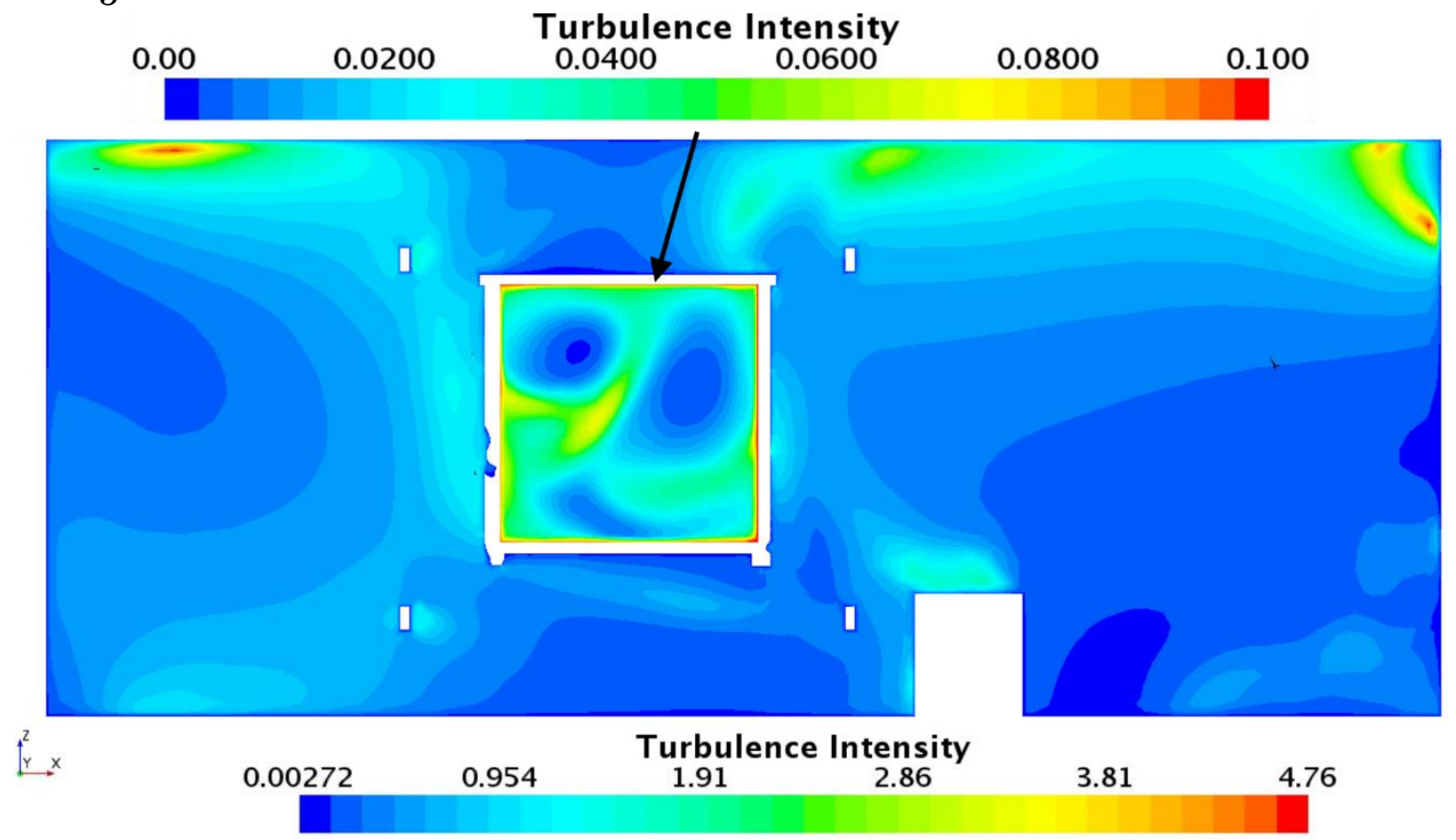


(b) $Y=1.5 \mathrm{in}$

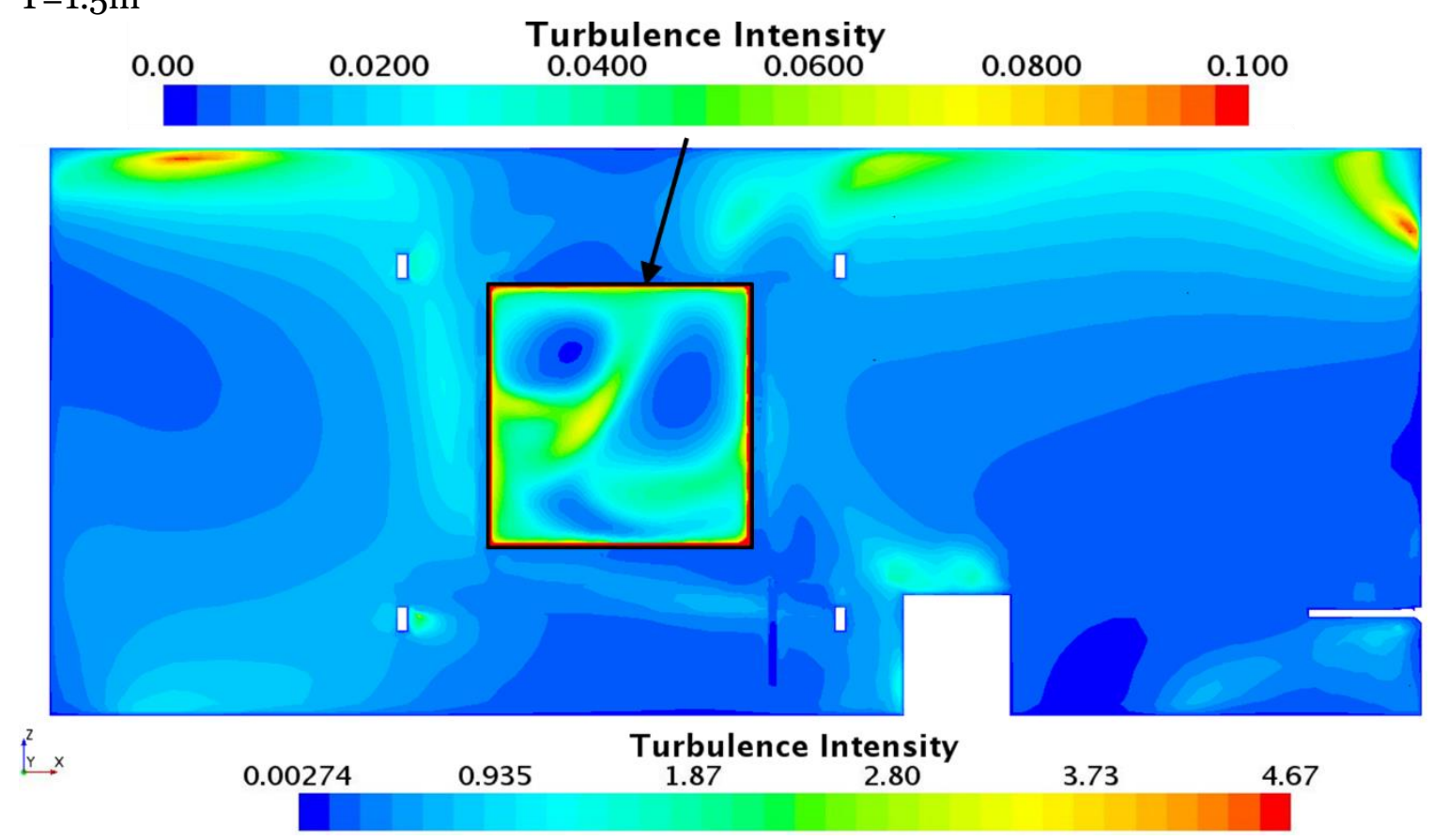

(c) $Y=1 \mathrm{ft}$

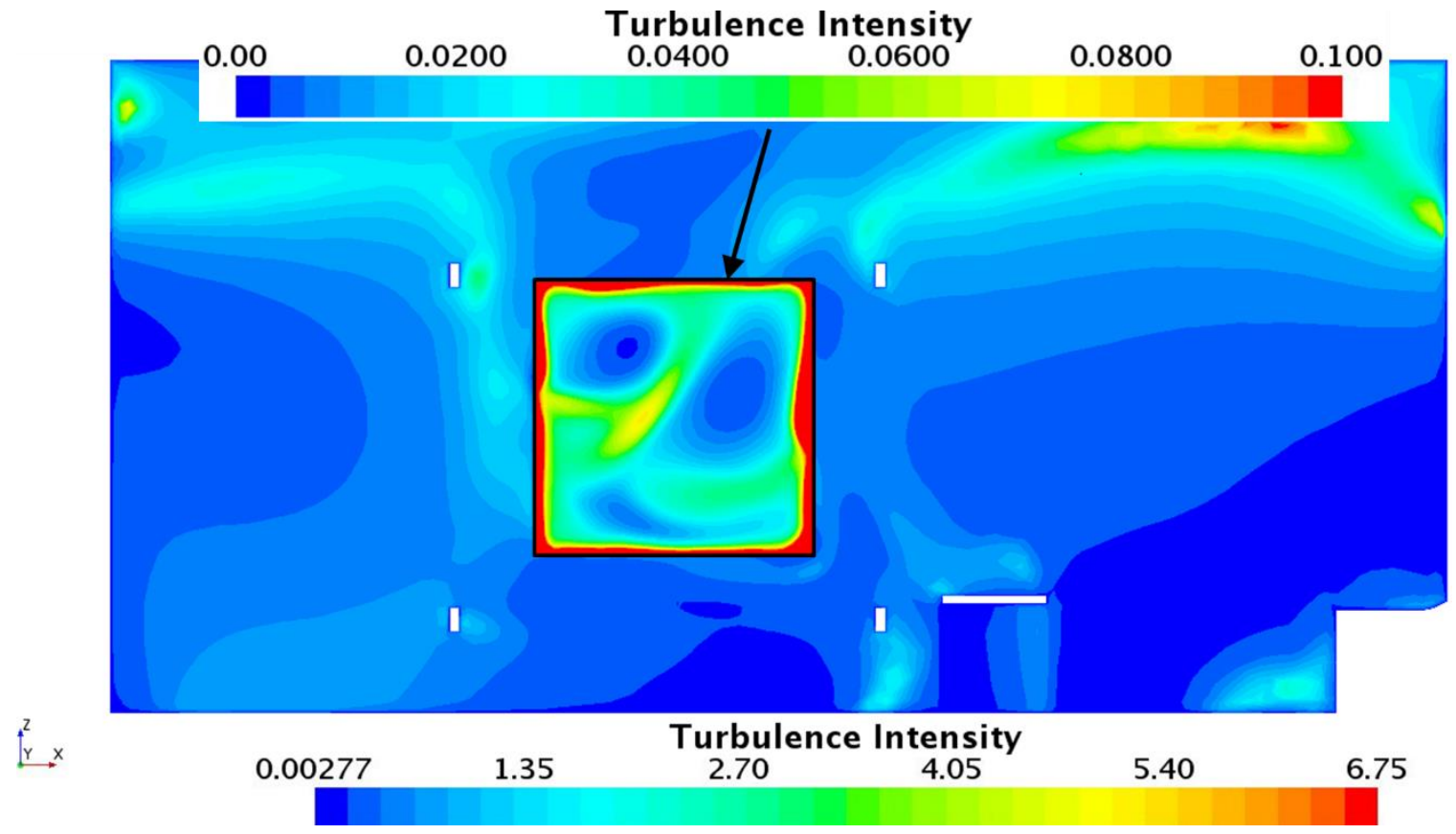


(d) $Y=2 f t$

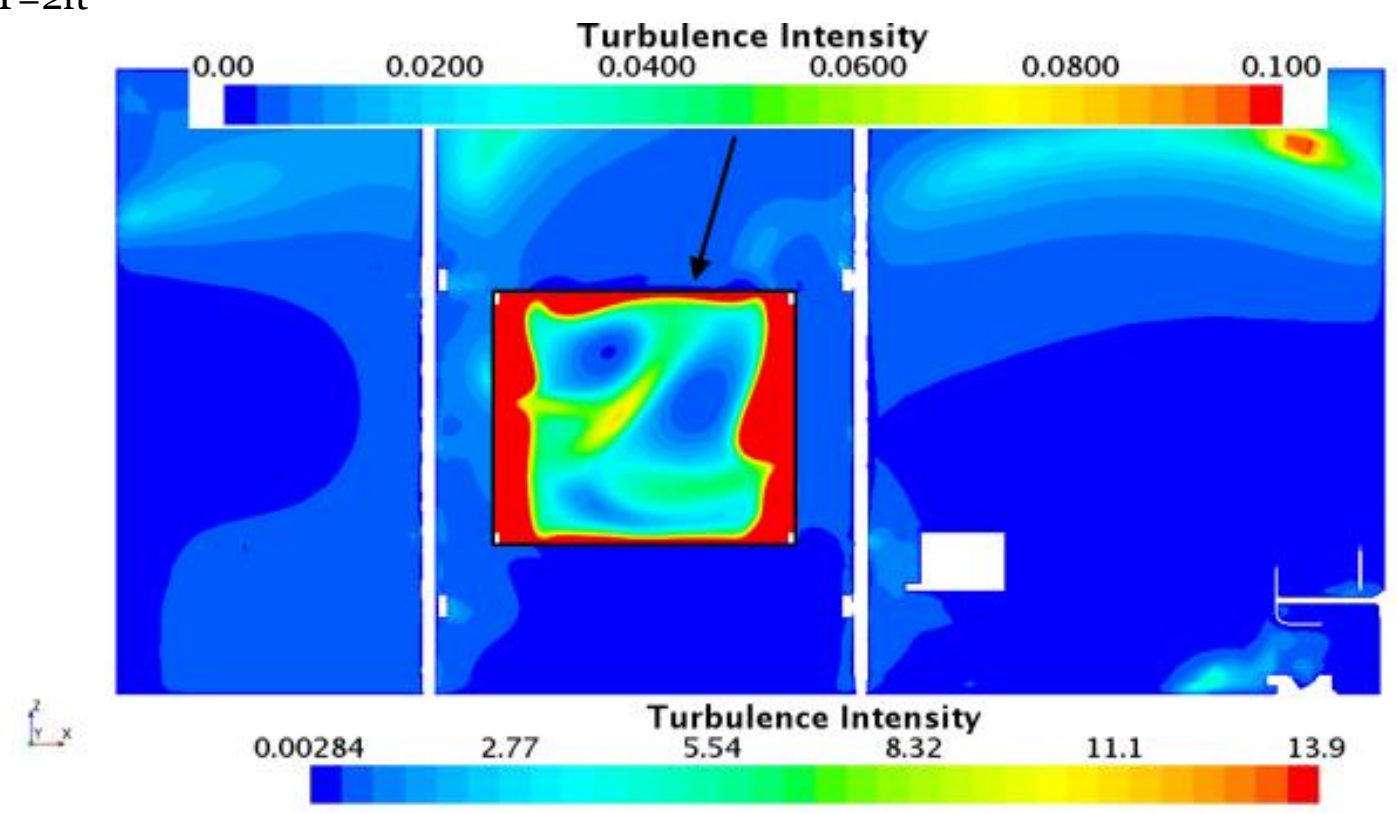

Figure 4-8: Turbulence intensity distribution on planes: (a) $Y=-1.5$ in, (b) $Y=1.5$ in, (c) $Y=1 \mathrm{ft}$, (d) $Y=2 \mathrm{ft}$. Model with rotating reference frame, RANS solver.

This full geometry model was run with different fan speeds, from $117 \mathrm{rpm}$ to $437 \mathrm{rpm}$. The velocities at the last screen were established and a relationship between them and the fan speed was calculated and plotted in Figure 4-9. These velocity values were later used as inlet velocity in the simplified models.

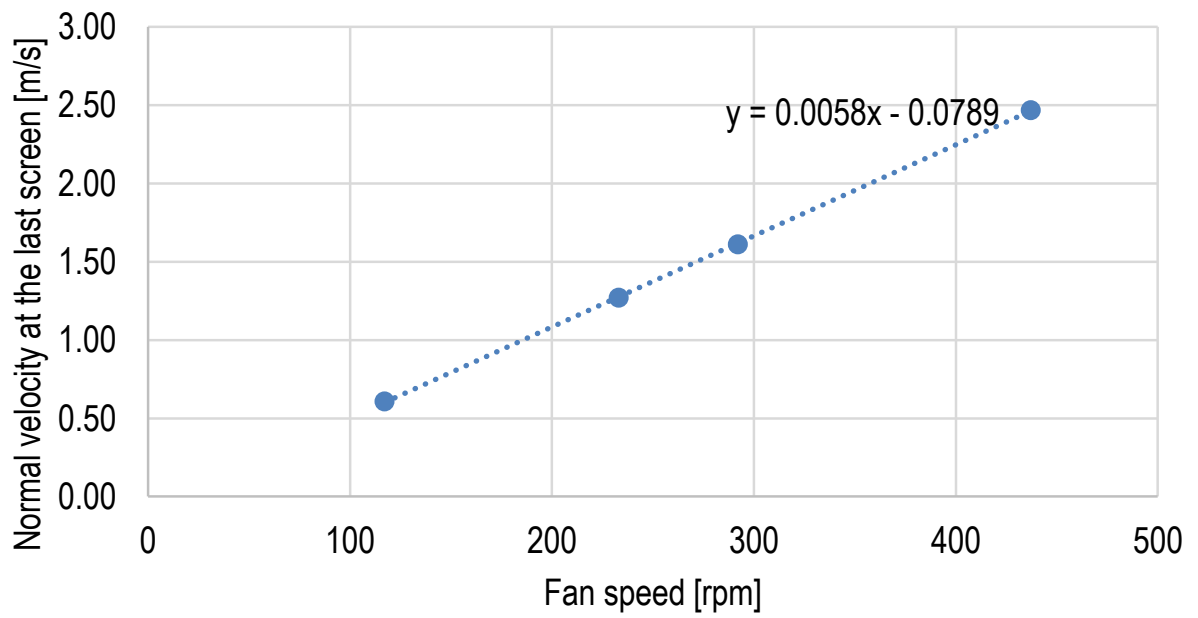

- CFD-steady RANS $\quad$ …..... Linear (CFD-steady RANS)

Figure 4-9: Relationship between the average normal velocity at the last screen in the wind tunnel and the fan speed. A linear fit was shown. Model with rotating reference frame, RANS solver. 


\subsubsection{Large Eddy Simulations}

The same CFD model was run with the LES solver. Figure 4-10 shows the velocity distribution in the domain. A turbulent flow was noticed around the fan, with numerous eddies, which are smoothed out by the wind tunnel screens. The air flowing out of the wind tunnel exit was much more uniform. Eddies formed in the downwind part of the jet, as the air was deflected by the wall. It can be seen in Figure 4-11 that the velocity in the $\mathrm{Y}$ or jet direction at point 20 oscillates with an amplitude of approximately $1 \mathrm{~m} / \mathrm{s}$, whereas the velocity at point 23 oscillates with the amplitude of about 3-4 m/s. Figure 4-12 shows typical instantaneous distributions of velocity in the $\mathrm{Y}$ direction on planes: (a) $\mathrm{Y}=-1.5 \mathrm{in}$, (b) $\mathrm{Y}=1.5 \mathrm{in}$, (c) $\mathrm{Y}=1 \mathrm{ft}$, and (d) $\mathrm{Y}=\mathbf{2} \mathrm{ft}$. Figure 4-13 presents typical instantaneous $Y$ velocity distributions on a vertical plane at (a) point 20, and (b) point 23.

(a)

(b)

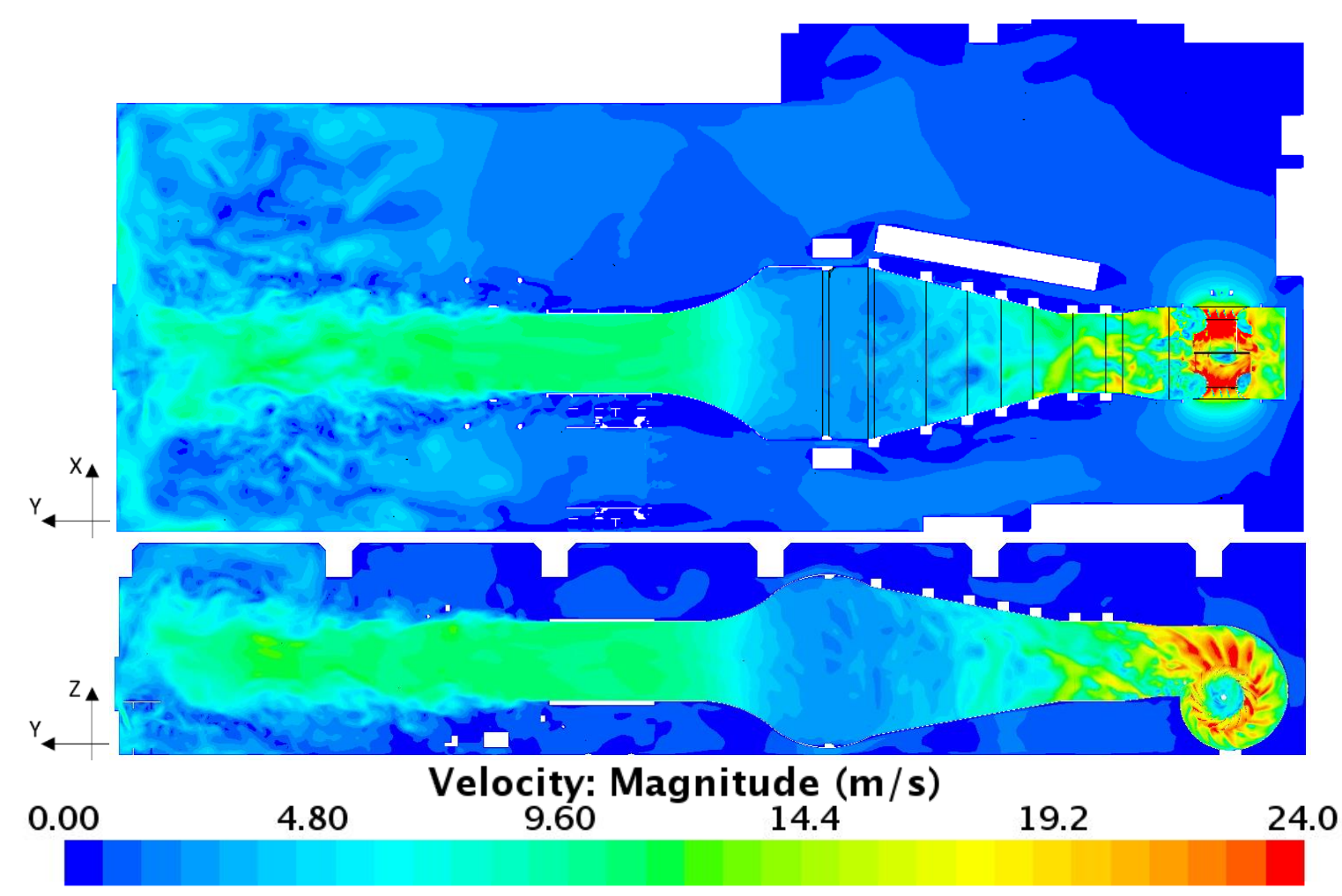

Figure 4-10: Velocity magnitude on a) horizontal plane, b) vertical plane. Model with rotating reference frame, LES solver. 


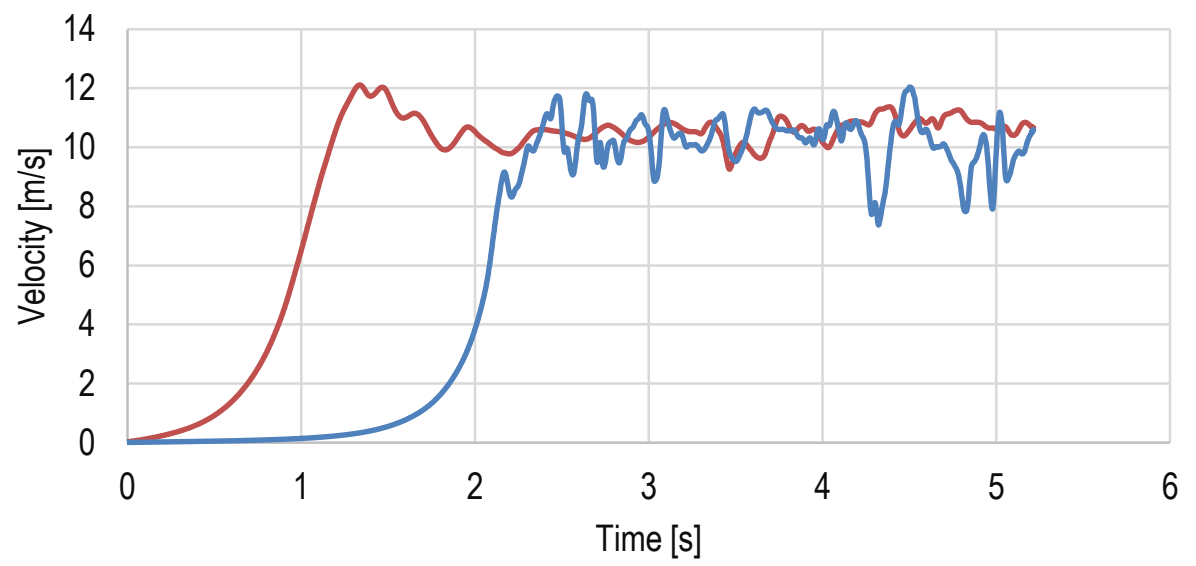

— velPt20 Monitor: velPt20 Monitor $(\mathrm{m} / \mathrm{s})$

— velPt23 Monitor: velPt23 Monitor $(\mathrm{m} / \mathrm{s})$

Figure 4-11: Velocity in Y direction at points 20 and 23. Model with rotating reference frame, LES solver.

(a) $Y=-1.5$ in

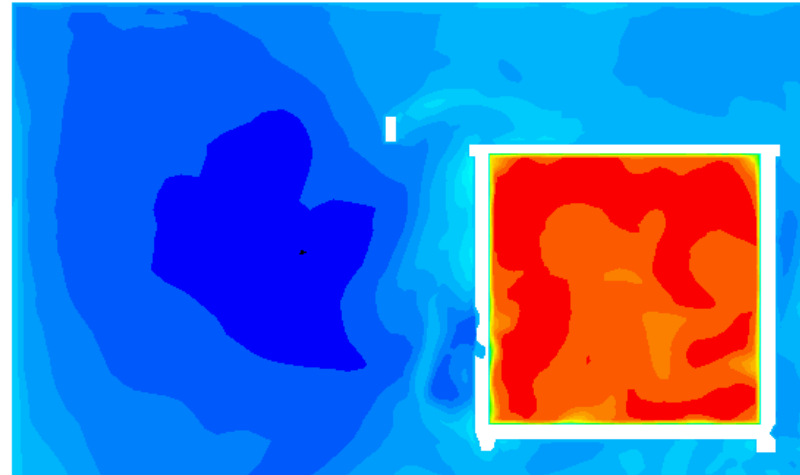

1
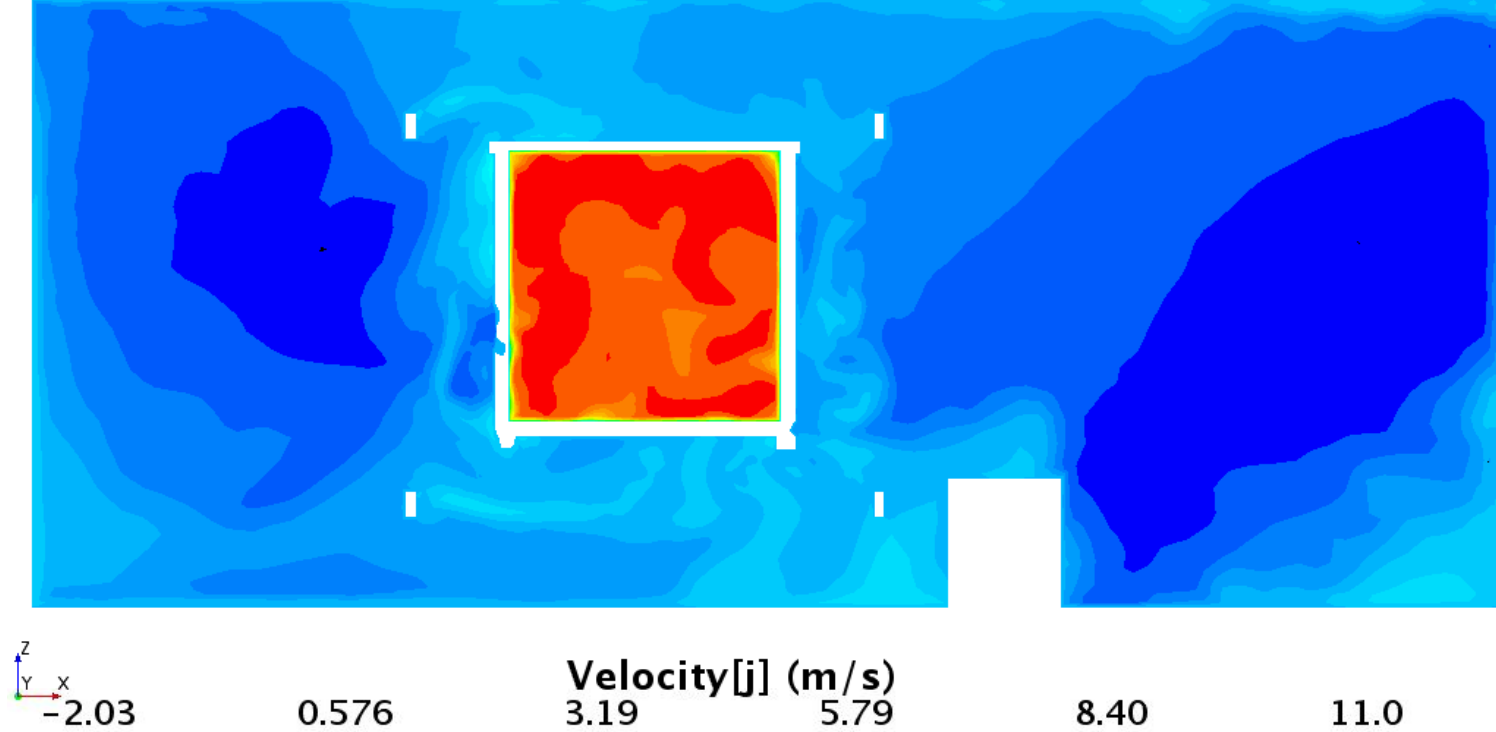

Velocity[j] $(\mathrm{m} / \mathrm{s})$

$3.19 \quad 5.79$

8.40

11.0 
(b) $Y=1.5$ in
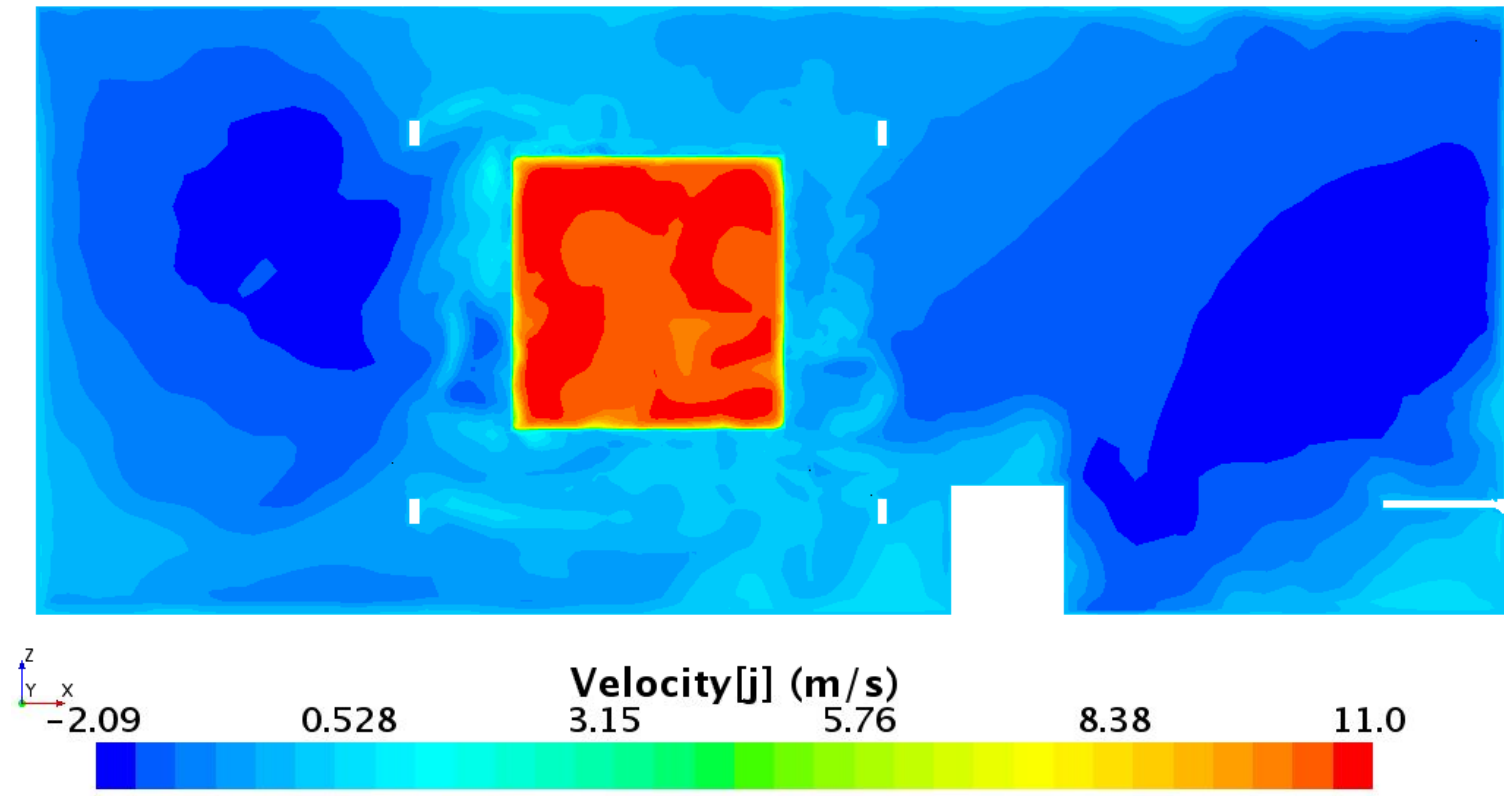

(c) $\mathrm{Y}=1 \mathrm{ft}$
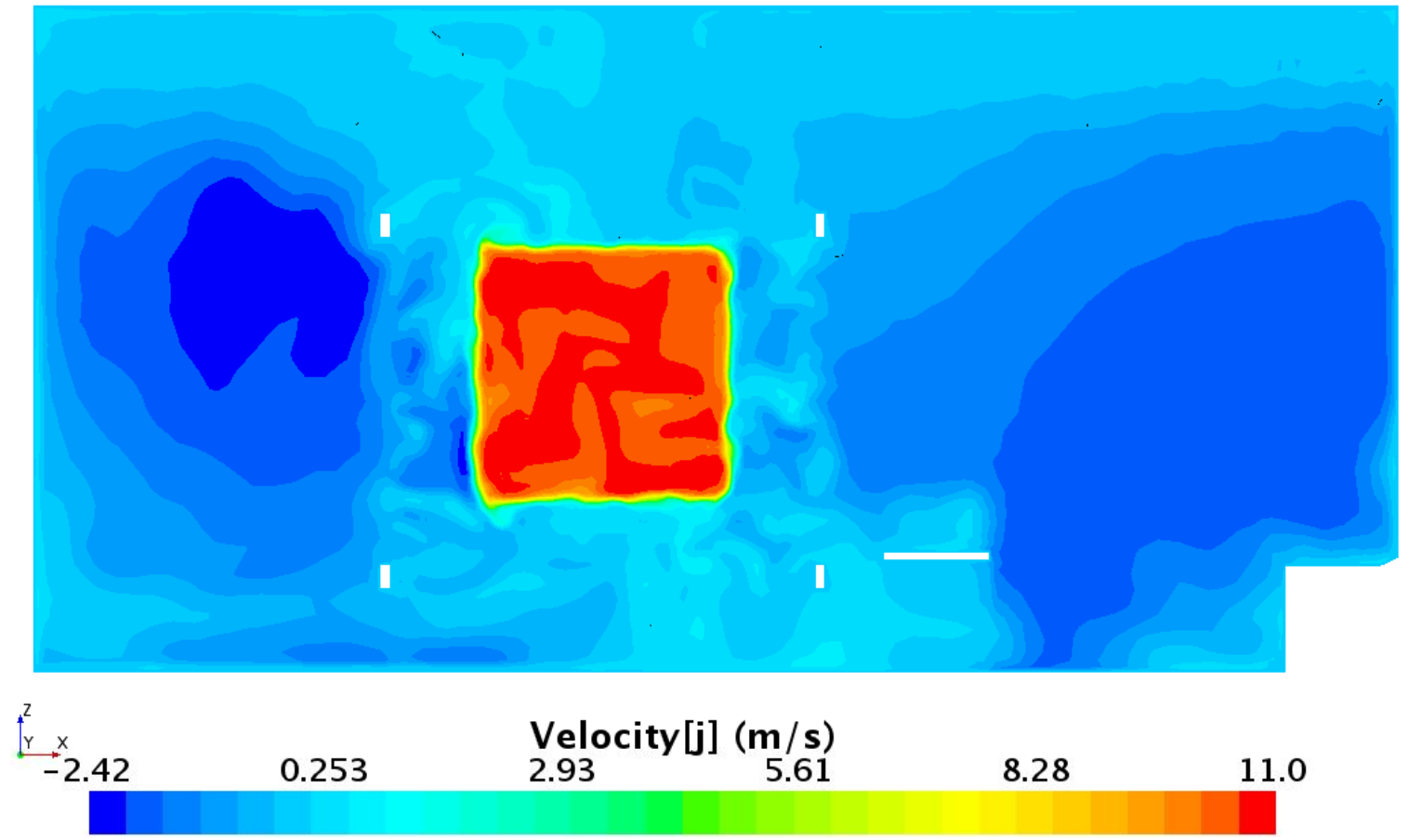
(d) $\quad \mathrm{Y}=2 \mathrm{ft}$
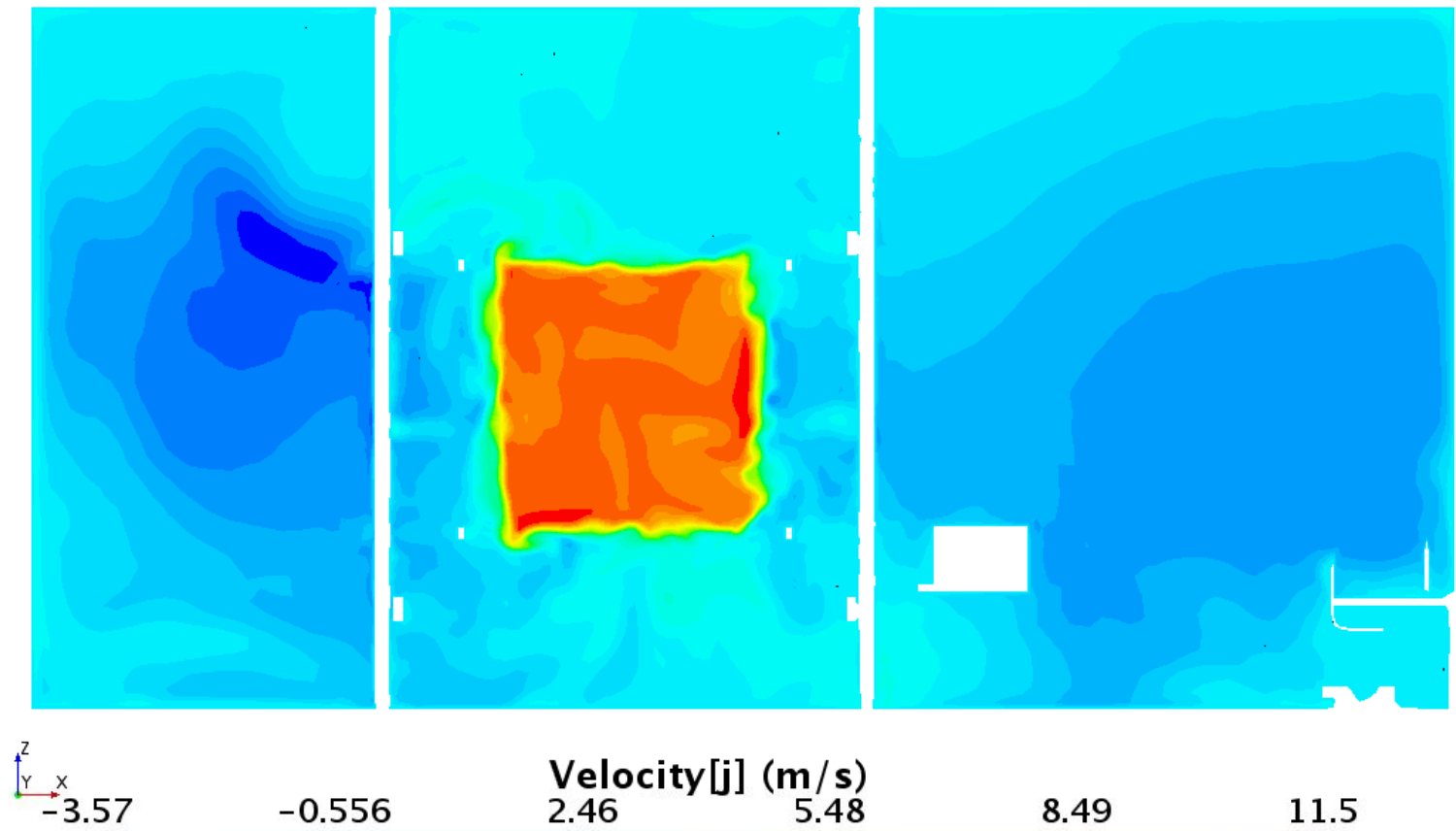

Velocity $[\mathrm{j}](\mathrm{m} / \mathrm{s})$

2.46

5.48

8.49

11.5

Figure 4-12: The distribution of velocity in $Y$ direction on planes: (a) $Y=-1.5$ in, (b) $Y=1.5$ in, (c) $Y=1 \mathrm{ft}$, (d) $\mathrm{Y}=2 \mathrm{ft}$. Model with rotating reference frame, LES solver.

(a)

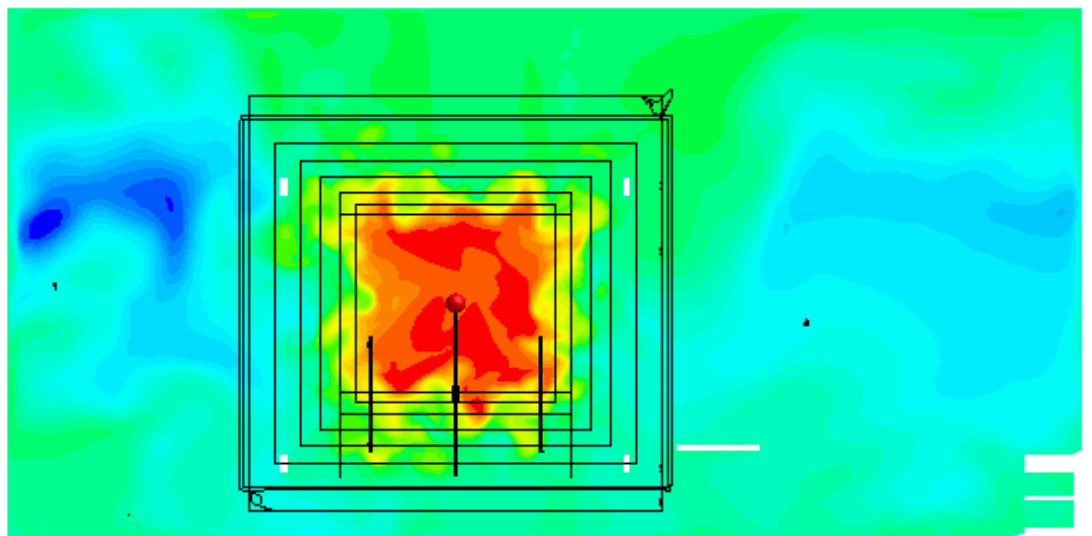

\begin{tabular}{lllll}
\multicolumn{1}{c}{ Velocity $[j](\mathrm{m} / \mathrm{s})$} & & \\
-7.68 & -3.83 & 0.0204 & 7.73 & 11.6
\end{tabular}


(b)

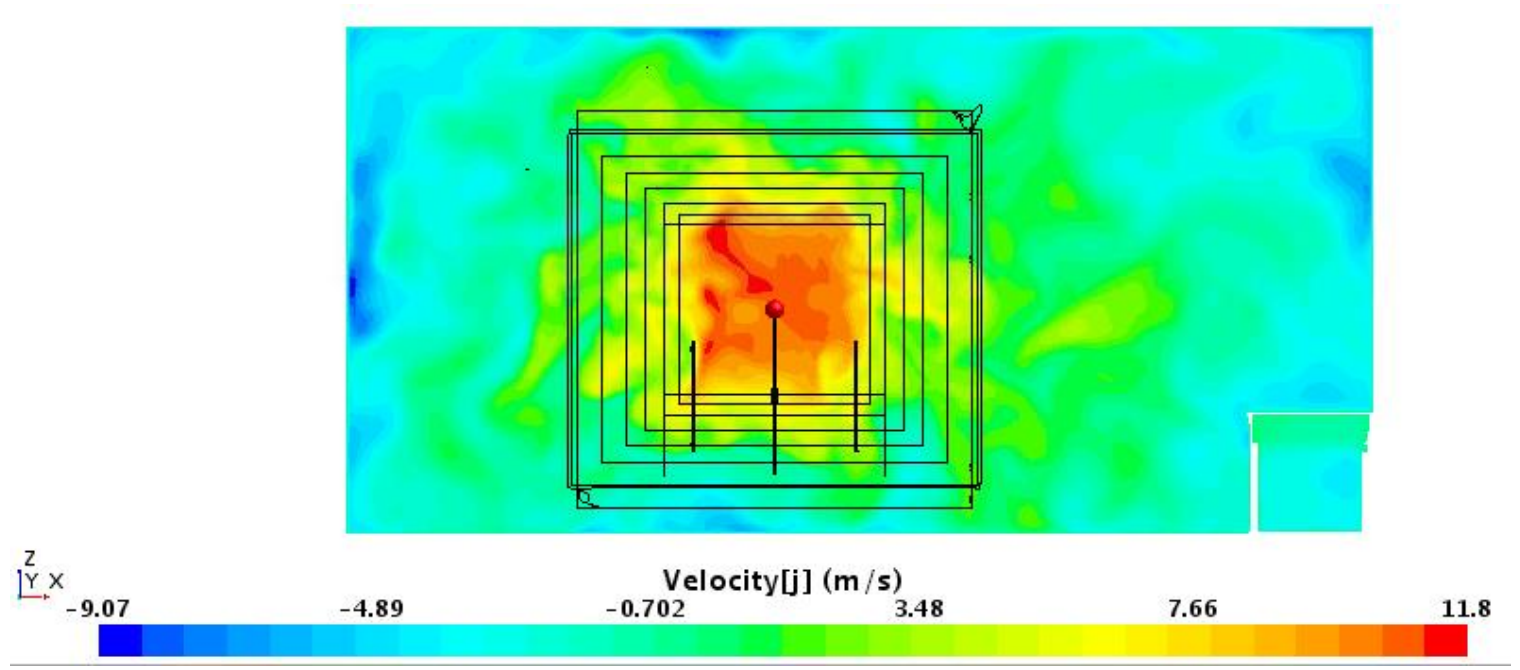

Figure 4-13: The distribution of velocity in Y direction on a vertical plane of (a) point 20, (b) point 23.

Model with rotating reference frame, LES solver.

\subsection{Results obtained with simplified models}

\subsubsection{RANS, k- $\varepsilon$ turbulence model}

The full model with rotating reference frame was run with different fan speeds. In every simulation, the velocities at the last screen were recorded. They are used as inlet velocities for the simplified model. The relationship between the fan speed and inlet velocity is close to linear, as shown in Figure 4-9. The ratio of these velocities equals 4.48 on average. A linear fit of the data was used to calculate inlet velocities for the simplified model, which would correspond to various fan speeds, and also those exceeding fan speeds used in the experiments.

This model has much faster convergence. Velocity magnitude at points 20 and 23 was monitored. The variables converged in about a 1000 iterations, and the computations took 1.5 hours on 4 nodes with 16 cores per node. A plot of the velocity vs. iteration number is shown in Figure 4-14. Also, the velocity values are closer to the mean values of the experimental results, see Table 2-1 and Table 2-2. 


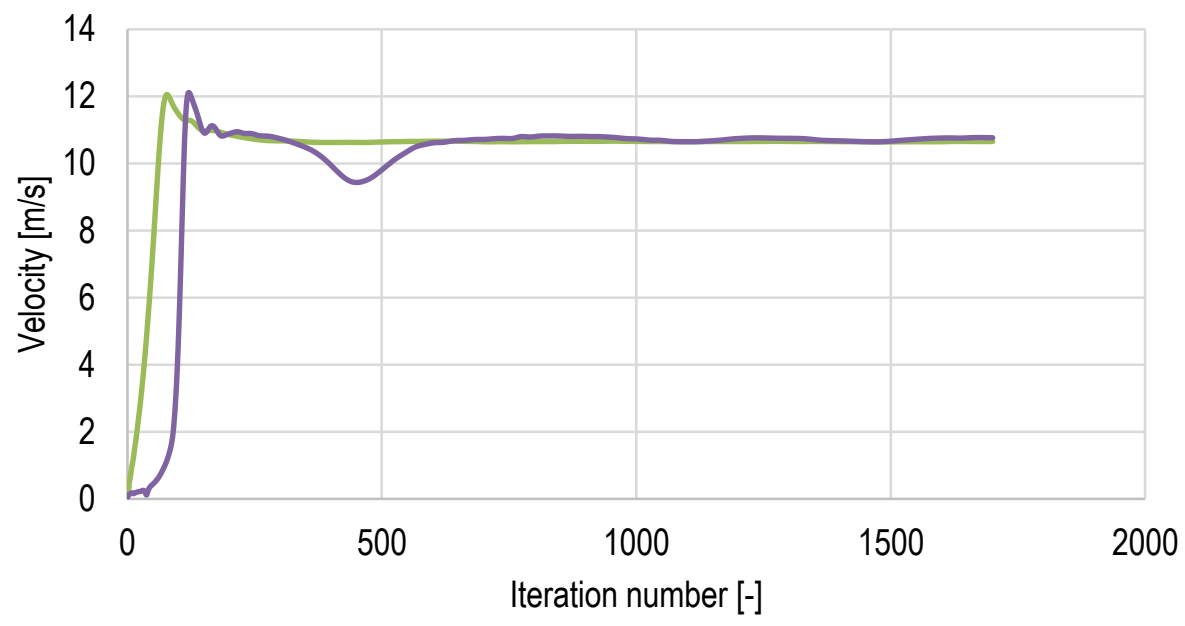

Point $20 \longrightarrow$ Point 23

Figure 4-14: Velocity vs iterations at points 20 and 23 during the simulation. Model with simplified geometry, RANS solver.

(a)

(b)

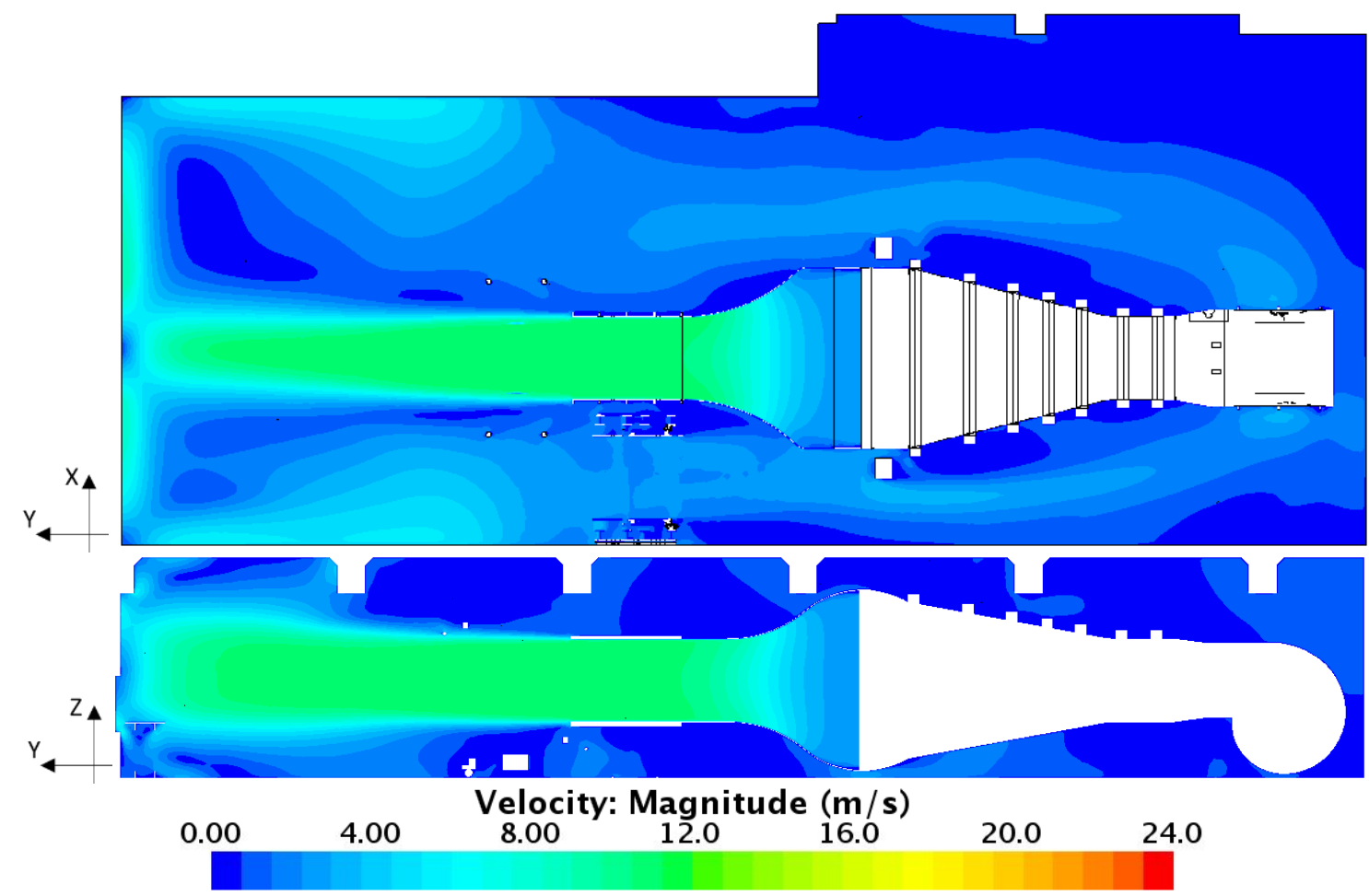

Figure 4-15: Velocity magnitude distribution on (a) horizontal plane, (b) vertical plane. Model with simplified geometry, RANS solver. 
(a) $Y=-1.5$ in

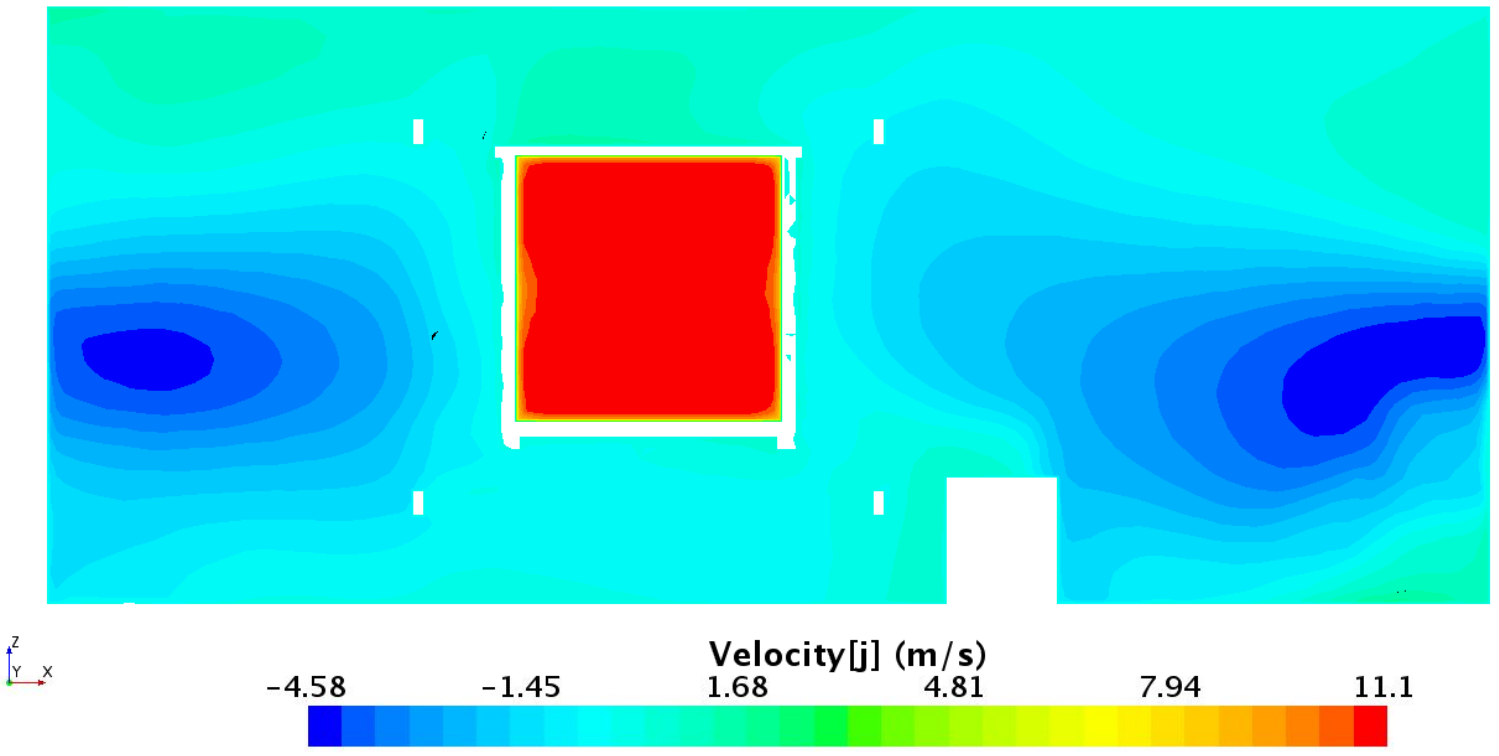

(b) $Y=1.5$ in

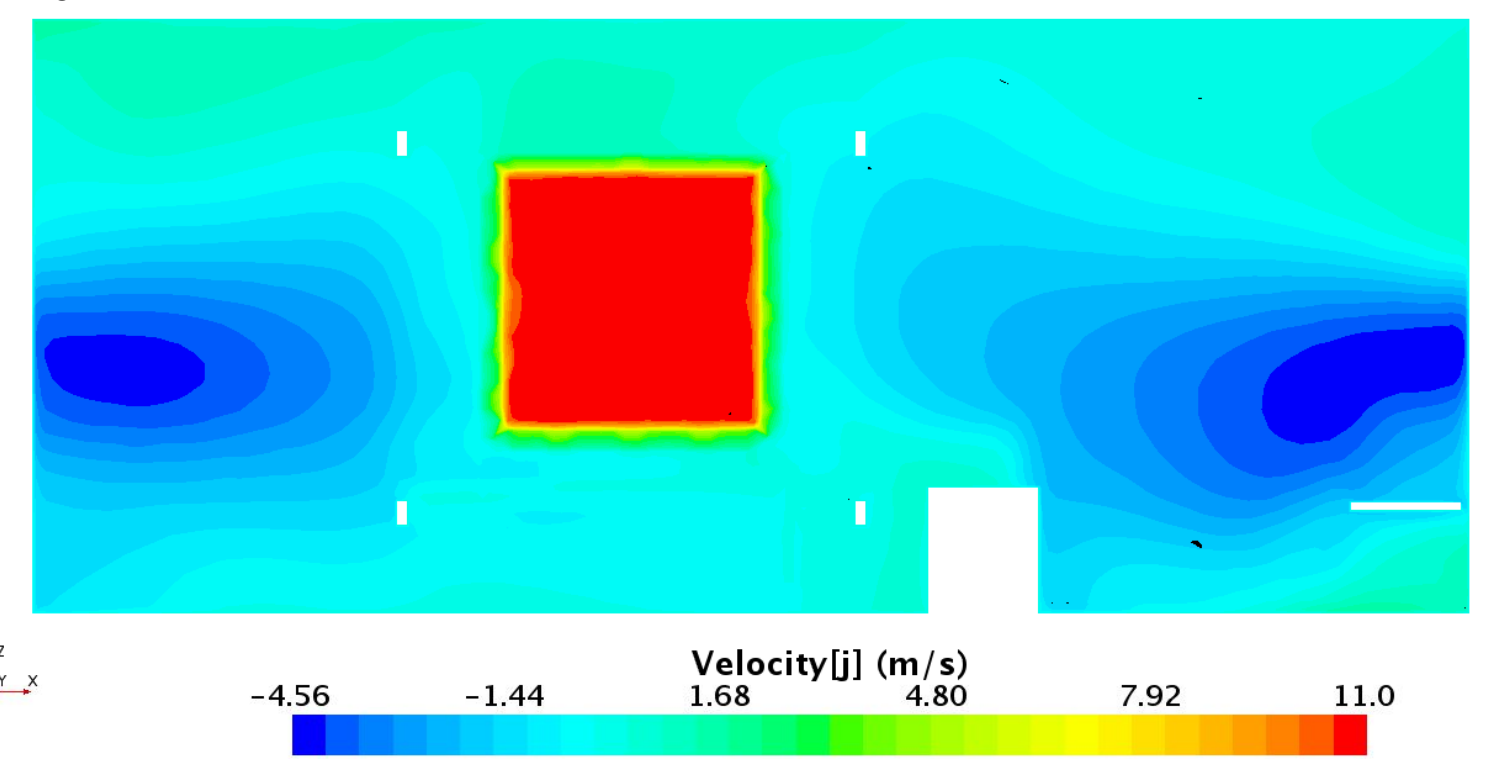


(c) $Y=1 \mathrm{ft}$

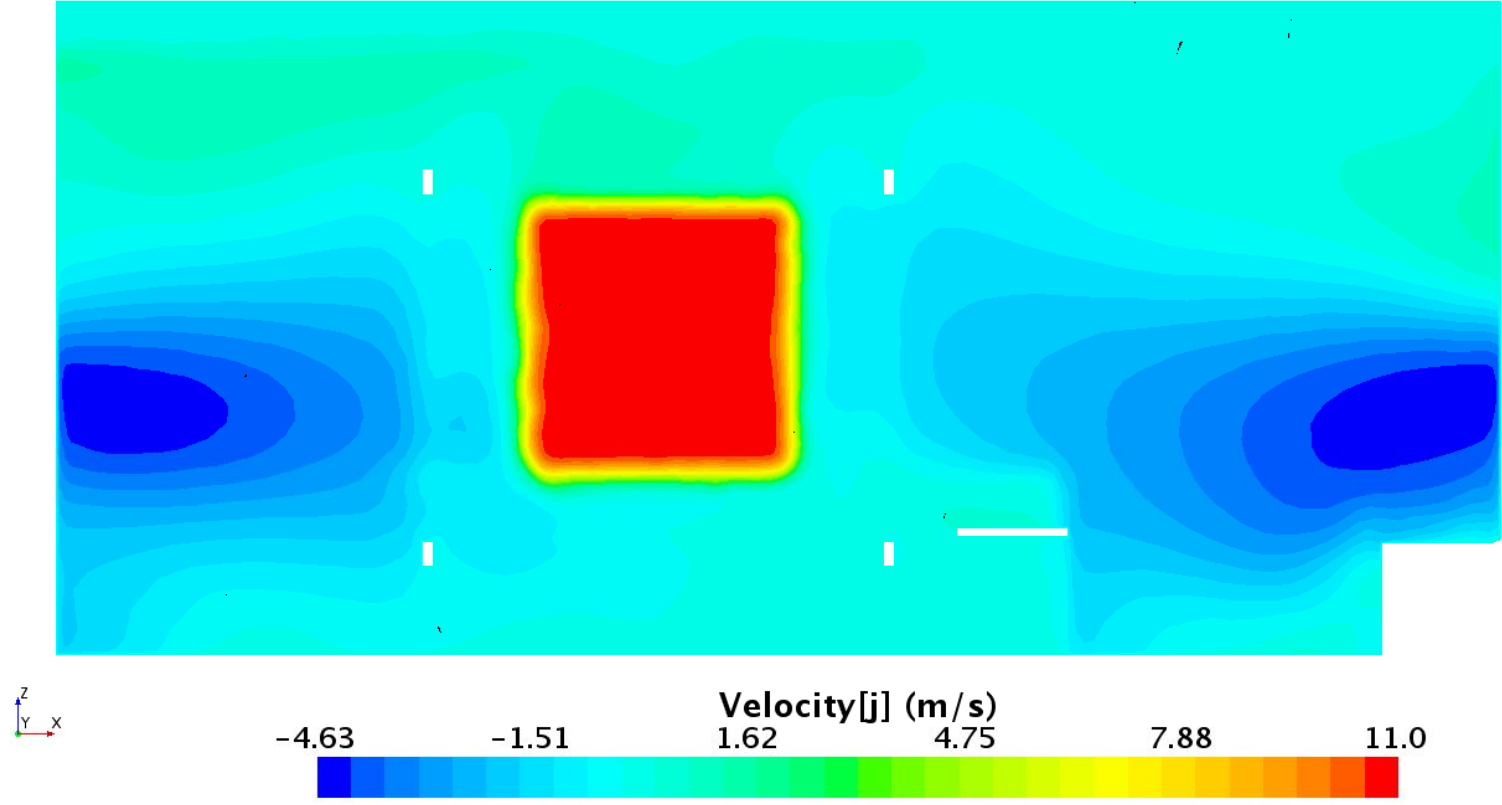

(d) $Y=2 f t$

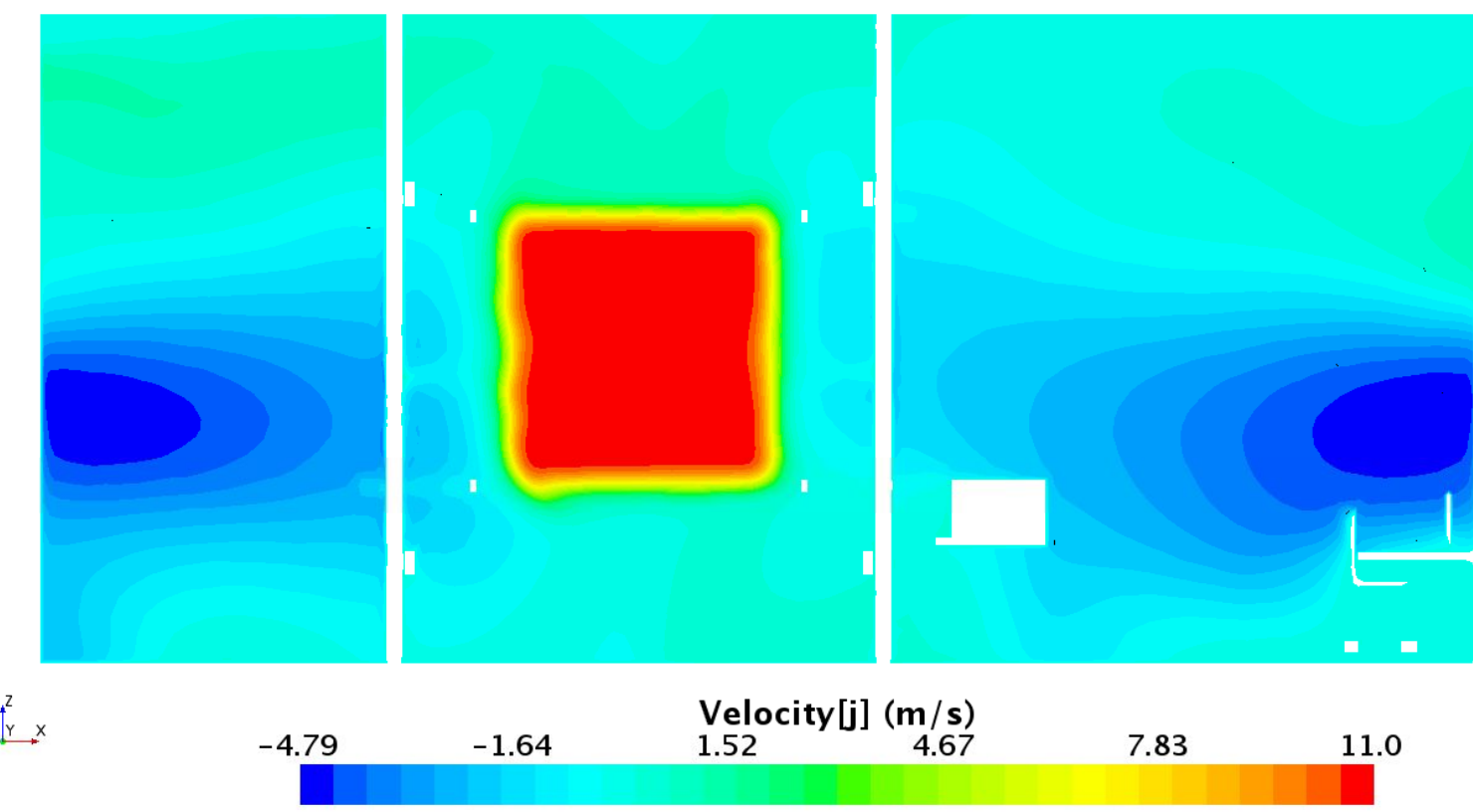

Figure 4-16: The distribution of velocity in $Y$ direction on planes: (a) $Y=-1.5$ in, (b) $Y=1.5$ in, (c) $Y=1 \mathrm{ft}$, (d) $\mathrm{Y}=2 \mathrm{ft}$. Model with simplified geometry, RANS solver. 
(a) $Y=-1.5 \mathrm{in}$

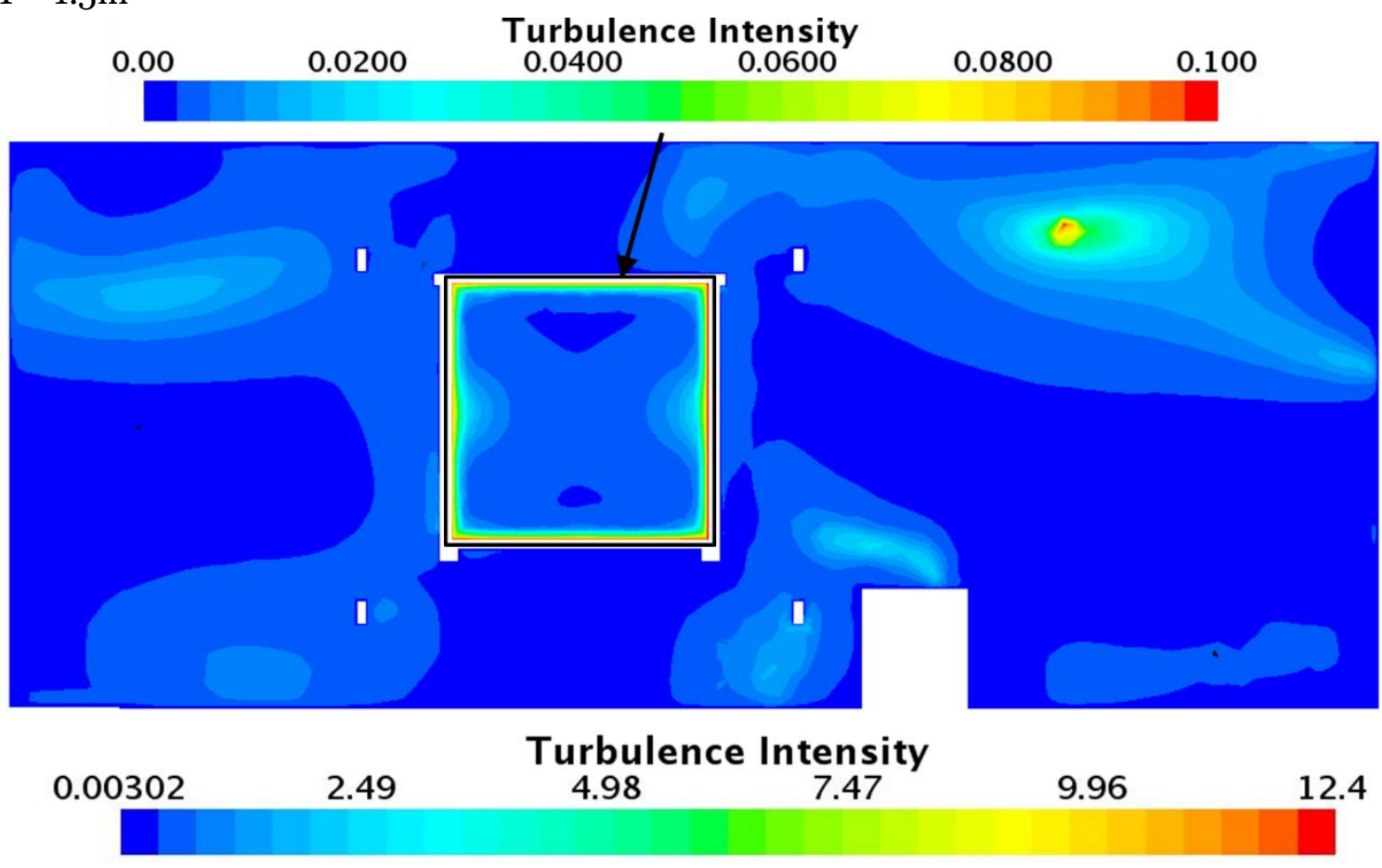

(b) $Y=1.5 \mathrm{in}$

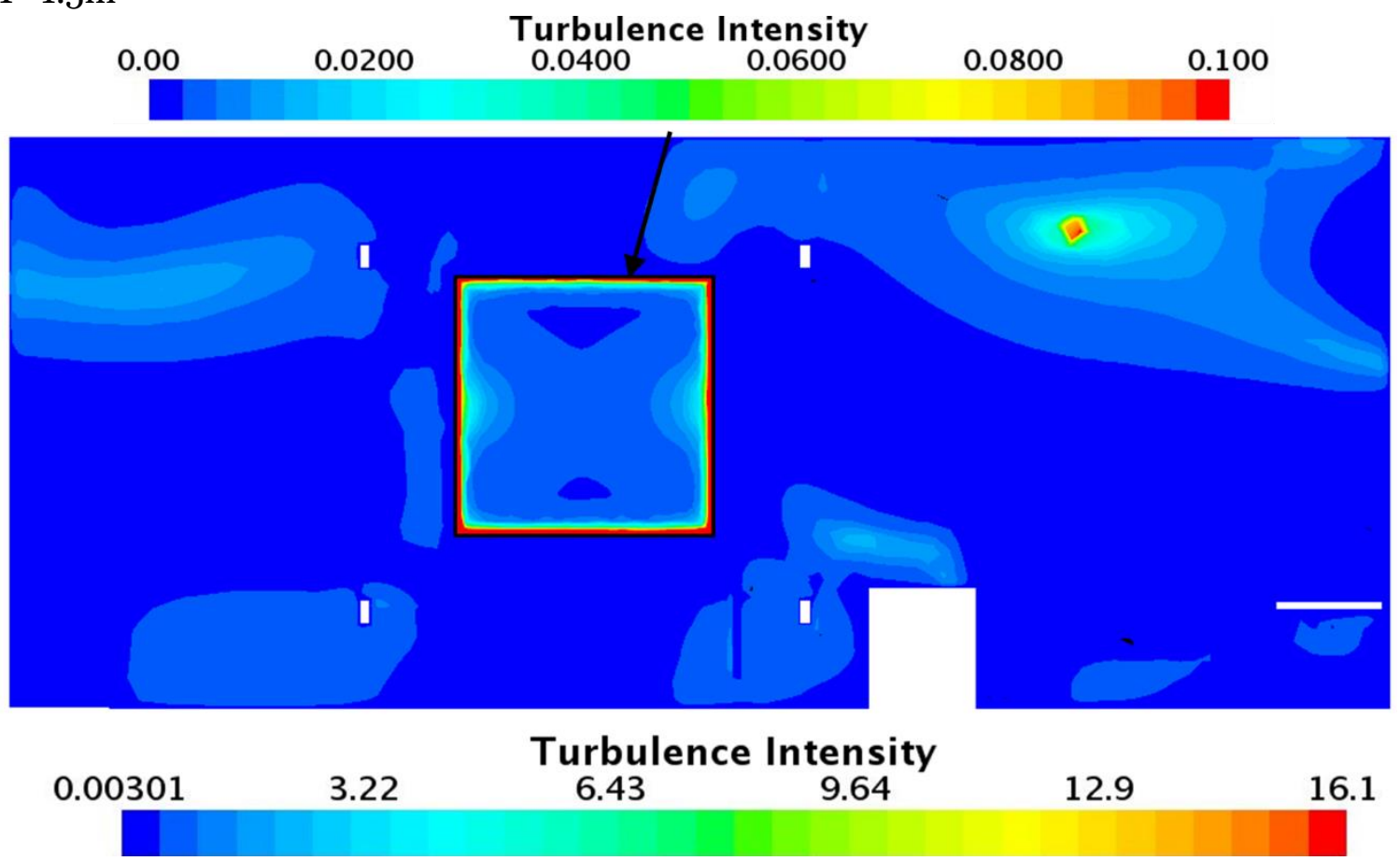


(c) $\mathrm{Y}=1 \mathrm{ft}$

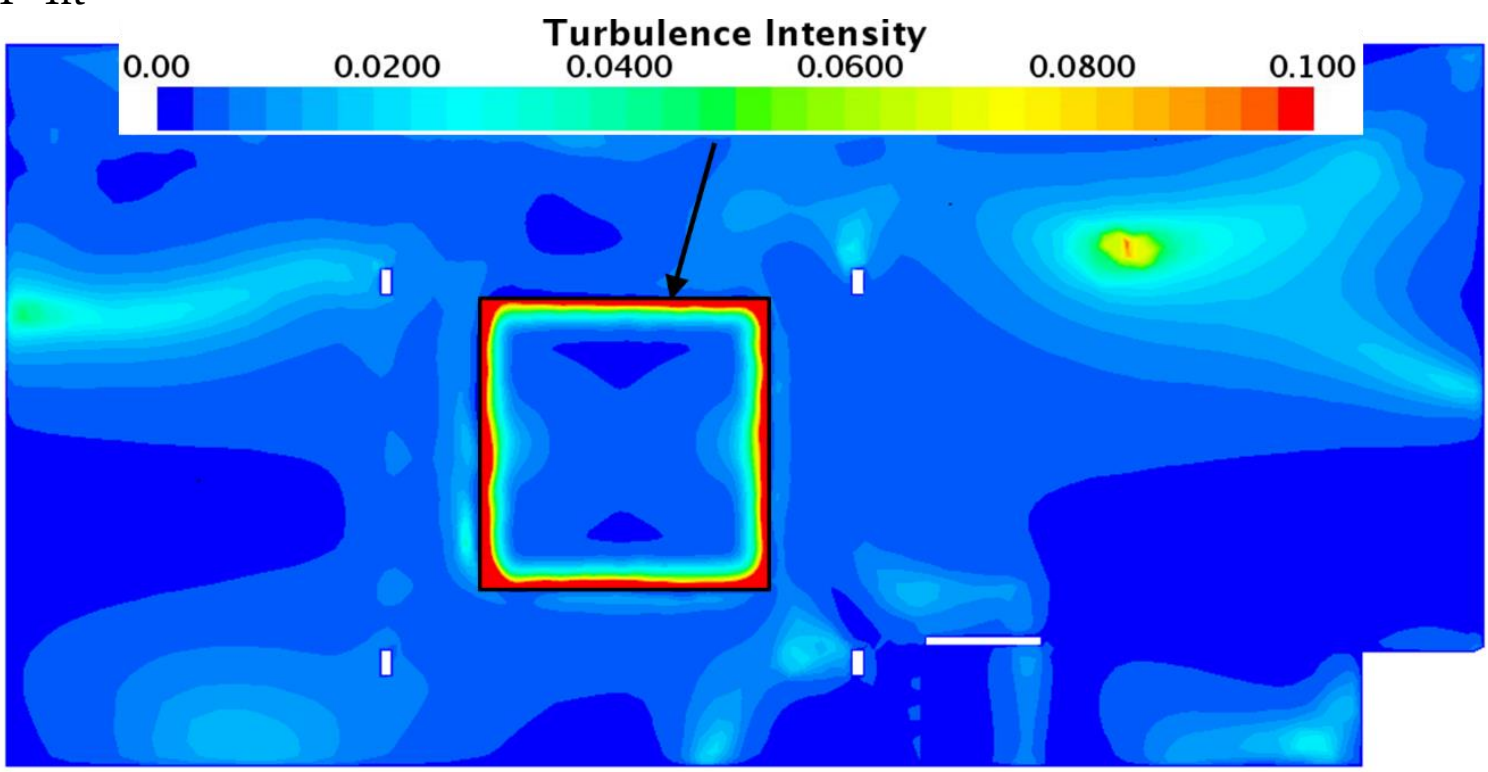

Turbulence Intensity

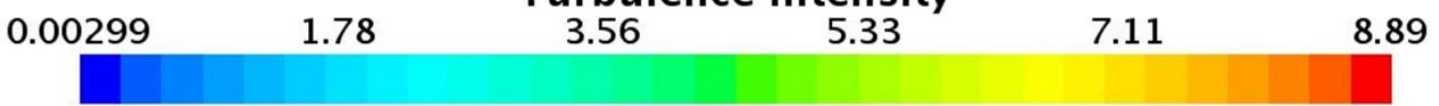

(d) $Y=2 f t$

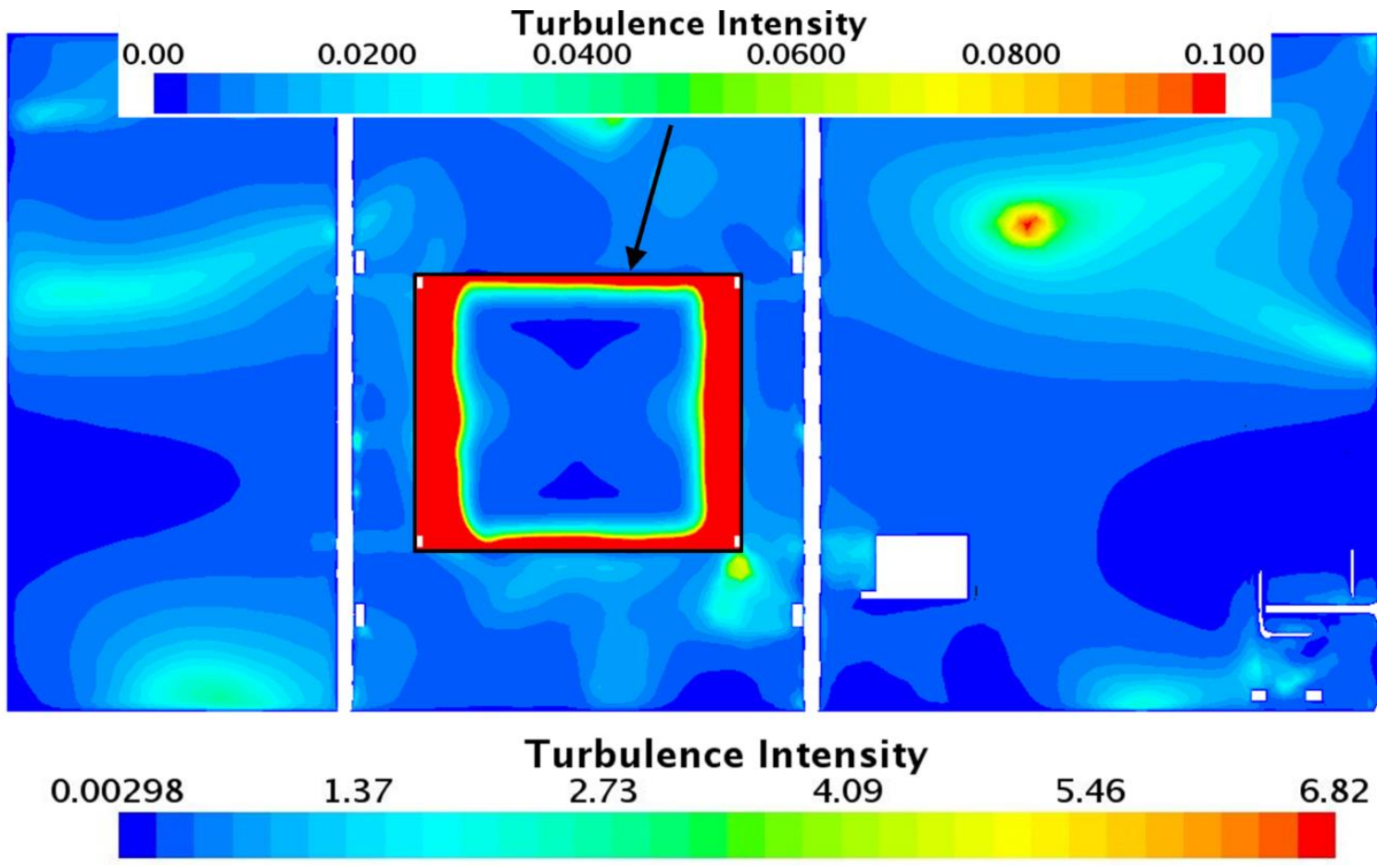

Figure 4-17: Turbulence intensity distribution on planes: (a) $Y=-1.5$ in, (b) $Y=1.5$ in, (c) $Y=1 \mathrm{ft}$, (d) $Y=2 \mathrm{ft}$. Model with simplified geometry, RANS solver. 
During the simulations, the velocity values at probe $1,2,3$, and the average from plane 4 were monitored. Figure 4-18 shows surface averaged velocities at probe plane 4 at various fan speeds. The plot includes values from measurements, a linear approximation of these results, and CFD results from the full model with RRF and the simplified model. The results from the simplified model were shown with $5 \%$ vertical error bars. A very good agreement with the approximation can be observed. Figure 4-19, Figure 4-20, and Figure 4-21 show a comparison of measured and computational velocities at Probe 1, 2, and 3 respectively, as well as linear fit of the experimental values. The vertical error bars for CFD results were shown as well. Table 4-3 illustrates the average velocities at probes 1, 2, and 3 obtained in laboratory measurements. A comparison of velocity values at Probe 1, 2, and 3 between the measurements and results obtained with the use of the model with simplified geometry were collected in Table 4-4. The relative error of velocities at Point 1, which was located in the center of the jet, and close to the wind tunnel exit, were the smallest and ranged from $4.7 \%$ for a fan speed of $100 \mathrm{rpm}$ to $5.9 \%$ for a fan speed of $500 \mathrm{rpm}$. The velocities at Probe 2 are overestimated, with the highest difference, $7.2 \%$, for the lowest fan speed. This point was located further away from the exit and the measurements exhibited the biggest variations. Point 3 was positioned inside the extension part of the tunnel. The computational results were underestimated there by up to $4 \%$ (at fan speed $500 \mathrm{rpm}$ ).

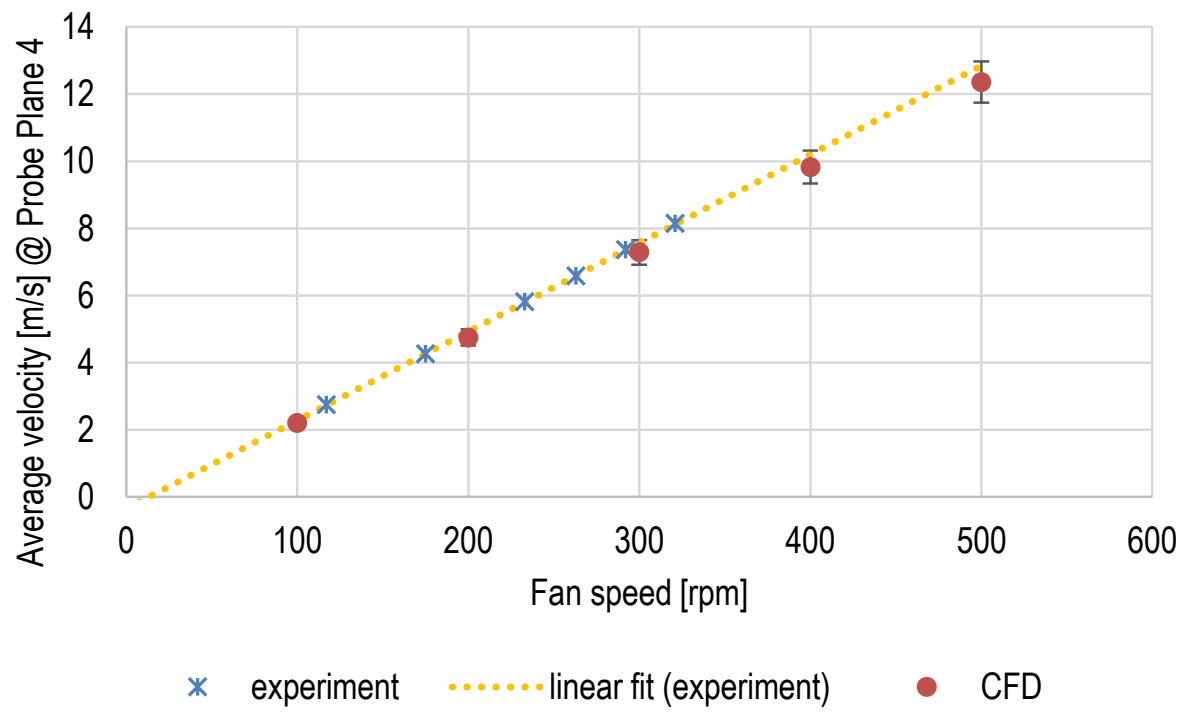

Figure 4-18: Average velocity at Probe 4 vs. fan speed. Model with simplified geometry, RANS solver. 


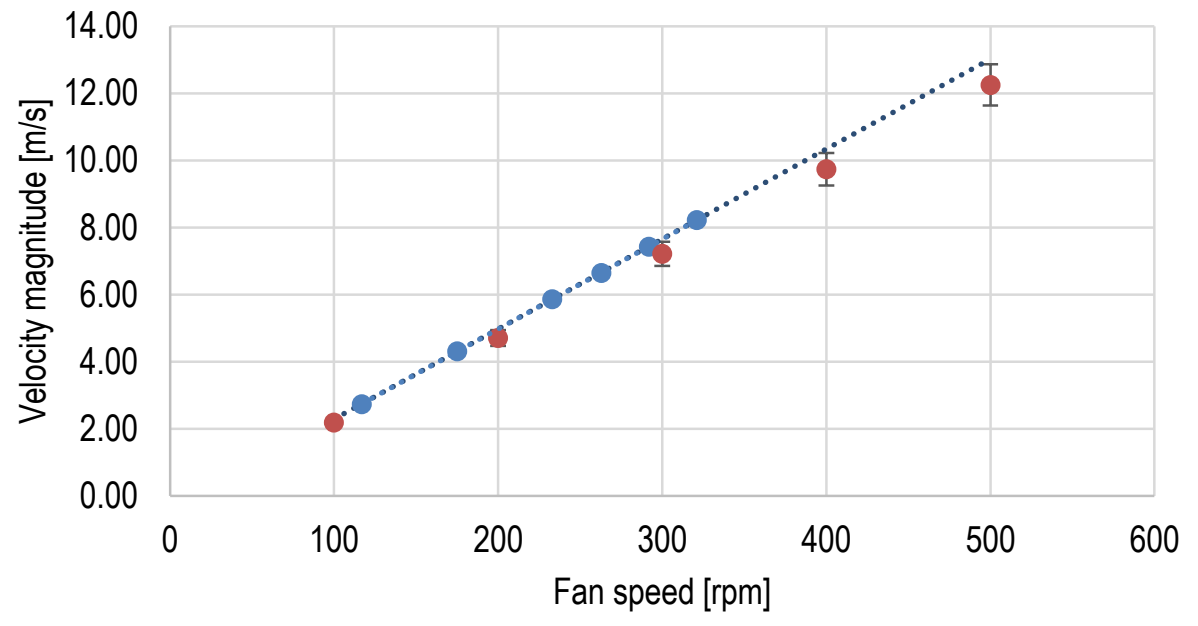

- experiment $\cdots . . . . .$. linear fit (experiment) $\quad$ CFD

Figure 4-19: Velocity at Probe 1 vs. fan speed. Model with simplified geometry, RANS solver.

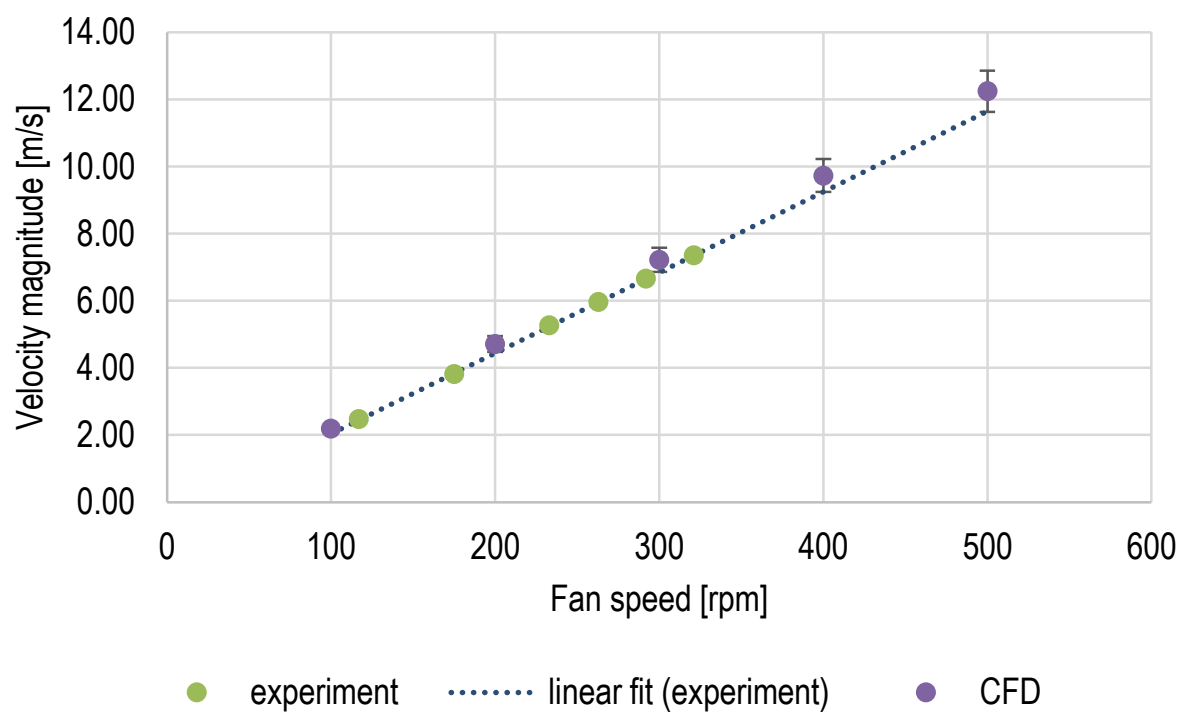

Figure 4-20: Velocity at Probe 2 vs. fan speed. Model with simplified geometry, RANS solver. 


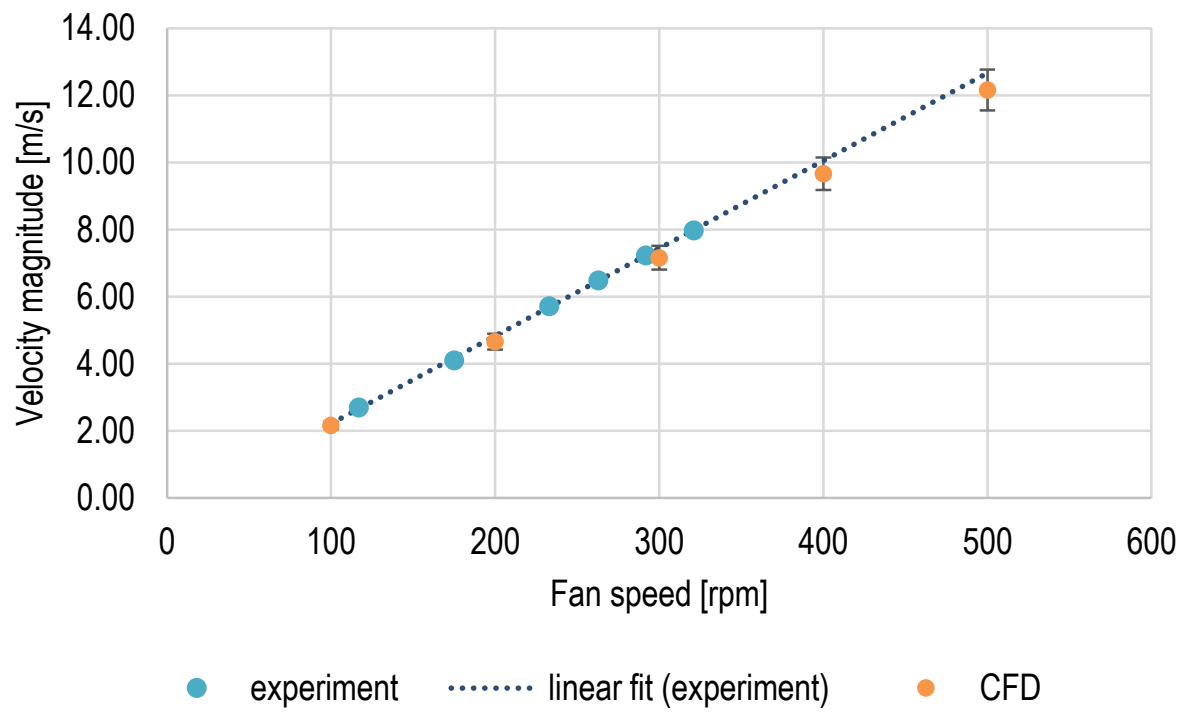

Figure 4-21: Velocity at Probe 3 vs. fan speed. Model with simplified geometry, RANS solver.

Table 4-3: Average flow velocity obtained in laboratory measurements at various fan speeds

\begin{tabular}{|c|c|c|c|c|c|c|c|}
\hline \multicolumn{2}{|c|}{ Fan speed rpm] } & $\mathbf{1 1 7}$ & $\mathbf{1 7 5}$ & $\mathbf{2 3 3}$ & $\mathbf{2 6 3}$ & $\mathbf{2 9 2}$ & $\mathbf{3 2 1}$ \\
\hline \multirow{2}{*}{$\begin{array}{c}\text { Average } \\
\text { velocity }\end{array}$} & Probe 1 & 2.74 & 4.32 & 5.86 & 6.65 & 7.43 & 8.22 \\
\cline { 2 - 8 }$[\mathbf{\text { Probe 2 }}]$ & Probe 3 & 2.47 & 3.81 & 5.26 & 5.97 & 6.66 & 7.36 \\
\cline { 2 - 8 } & 2.70 & 4.10 & 5.72 & 6.48 & 7.23 & 7.98 \\
\hline
\end{tabular}

Table 4-4: A comparison of velocity values at Probe 1, 2, and 3 between the measurements and results obtained with the use of the model with simplified geometry, RANS solver.

\begin{tabular}{|c|c|c|c|c|c|c|}
\hline \multicolumn{2}{|c|}{ Fan speed [rpm] } & 100 & 200 & 300 & 400 & 500 \\
\hline \multirow{3}{*}{$\begin{array}{c}\text { Linear fit of the } \\
\text { velocity } \\
\text { measurements } \\
\left(v_{\text {exp }}\right)[\mathrm{m} / \mathrm{s}]\end{array}$} & Probe 1 & 2.30 & 4.98 & 7.66 & 10.34 & 13.02 \\
\hline & Probe 2 & 2.04 & 4.44 & 6.84 & 9.24 & 11.64 \\
\hline & Probe 3 & 2.23 & 4.84 & $7 \cdot 45$ & 10.06 & 12.67 \\
\hline \multirow{3}{*}{$\begin{array}{c}\text { Velocity from } \\
\text { the simplified } \\
\text { model }\left(v_{C F D}\right) \\
{[\mathrm{m} / \mathrm{s}]}\end{array}$} & Probe 1 & 2.19 & 4.71 & 7.22 & 9.74 & 12.25 \\
\hline & Probe 2 & 2.19 & 4.71 & 7.22 & 9.73 & 12.24 \\
\hline & Probe 3 & 2.16 & 4.66 & 7.16 & 9.66 & 12.16 \\
\hline \multirow{3}{*}{$\begin{array}{c}\text { Relative } \\
\text { difference in } \\
\text { velocity, } \\
\left(\frac{v_{\text {exp }}-v_{C F D}}{v_{\text {exp }}}\right) \cdot 100 \%\end{array}$} & Probe 1 & $4 \cdot 7$ & 5.4 & $5 \cdot 7$ & 5.8 & $5 \cdot 9$ \\
\hline & Probe 2 & -7.2 & -6.0 & $-5 \cdot 5$ & $-5 \cdot 3$ & -5.1 \\
\hline & Probe 3 & 2.8 & 3.6 & 3.8 & $3 \cdot 9$ & 4.0 \\
\hline
\end{tabular}




\subsubsection{Large Eddy Simulations}

The CFD model with simplified geometry was run with the LES solver. The velocity distributions on a horizontal and a vertical plane in the domain at fan speed $437 \mathrm{rpm}$ are shown in Figure 4-22. The inlet parameters in this model were kept spatially and temporarily constant, therefore the flow inside the wind tunnel downstream of the inlet at the last screen, and its extension is uniform because there are no irregularities to produce eddies in that zone. A turbulent flow with eddy structures was noticed in the testing area and the rest of the room. Bigger eddies formed in the downwind part of the jet, as the air was deflected by the wall. Figure 4-23 shows the distribution of velocity in Y direction on vertical planes: (a) $Y=-1.5$ in, (b) $Y=1.5$ in, (c) $Y=1 \mathrm{ft}$, and (d) $Y=2 \mathrm{ft}$. Figure 4-24 presents a history plot of the $Y$ velocity at point 20, and point 23. Significant oscillations were recorded at the point closer to the wall (point 23).

(a)

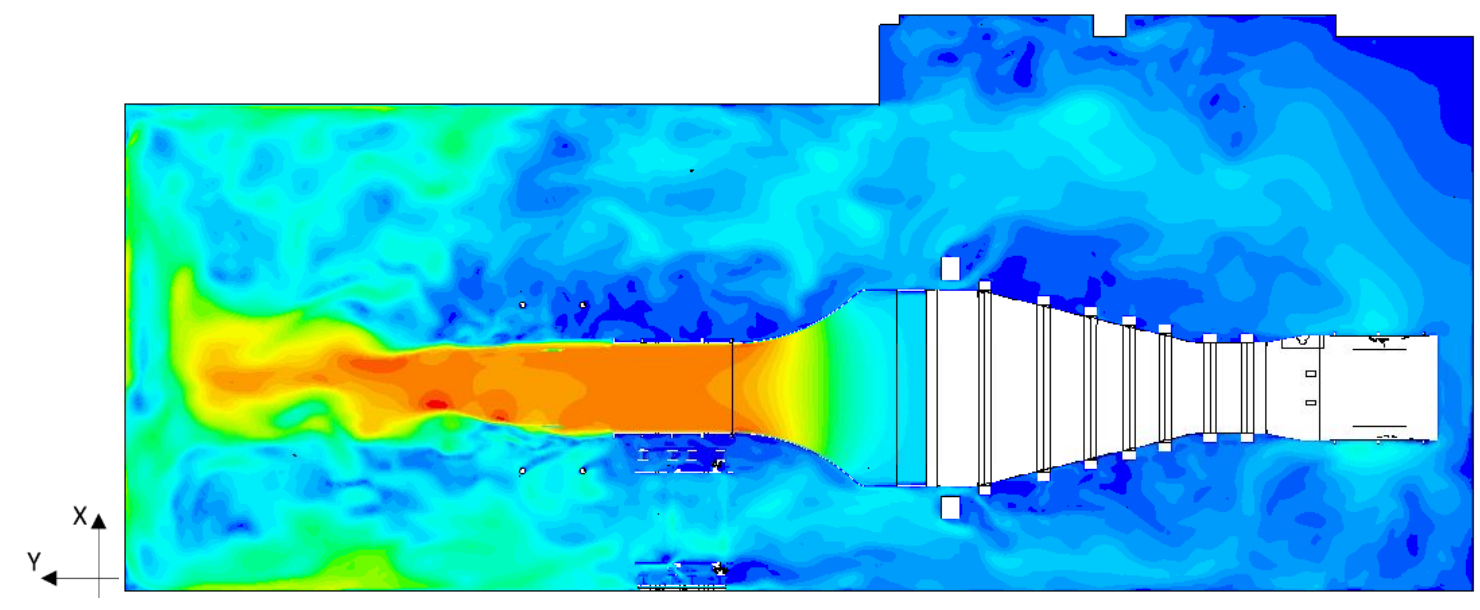

(b)

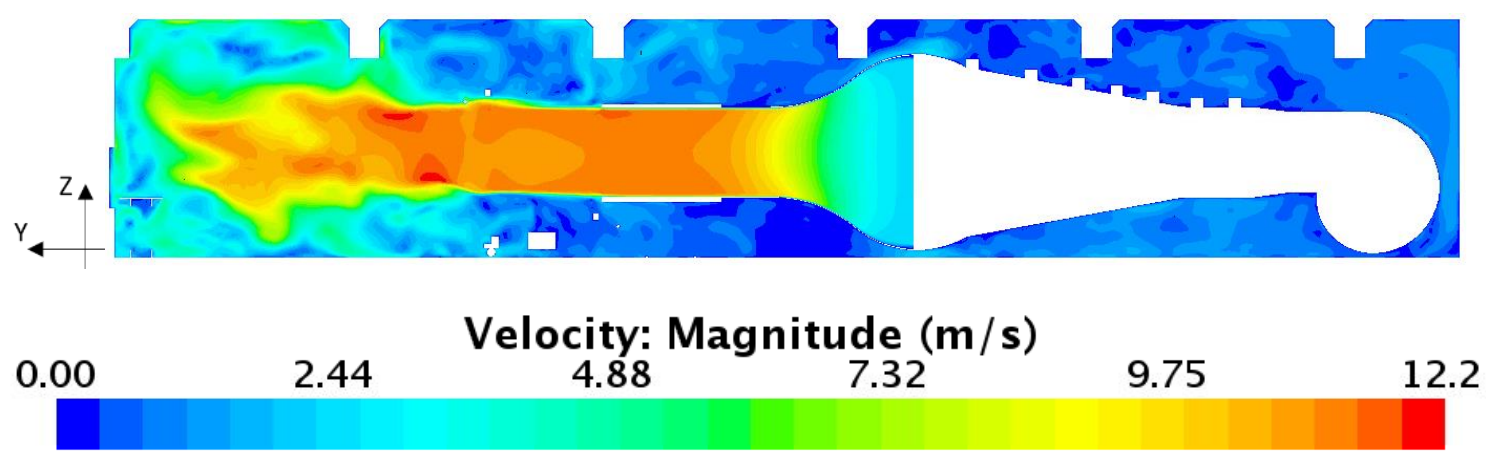

Figure 4-22: Velocity magnitude distribution on (a) horizontal, (b) vertical planes. Model with simplified geometry, LES solver. 
(a) $\mathrm{Y}=-1.5 \mathrm{in}$

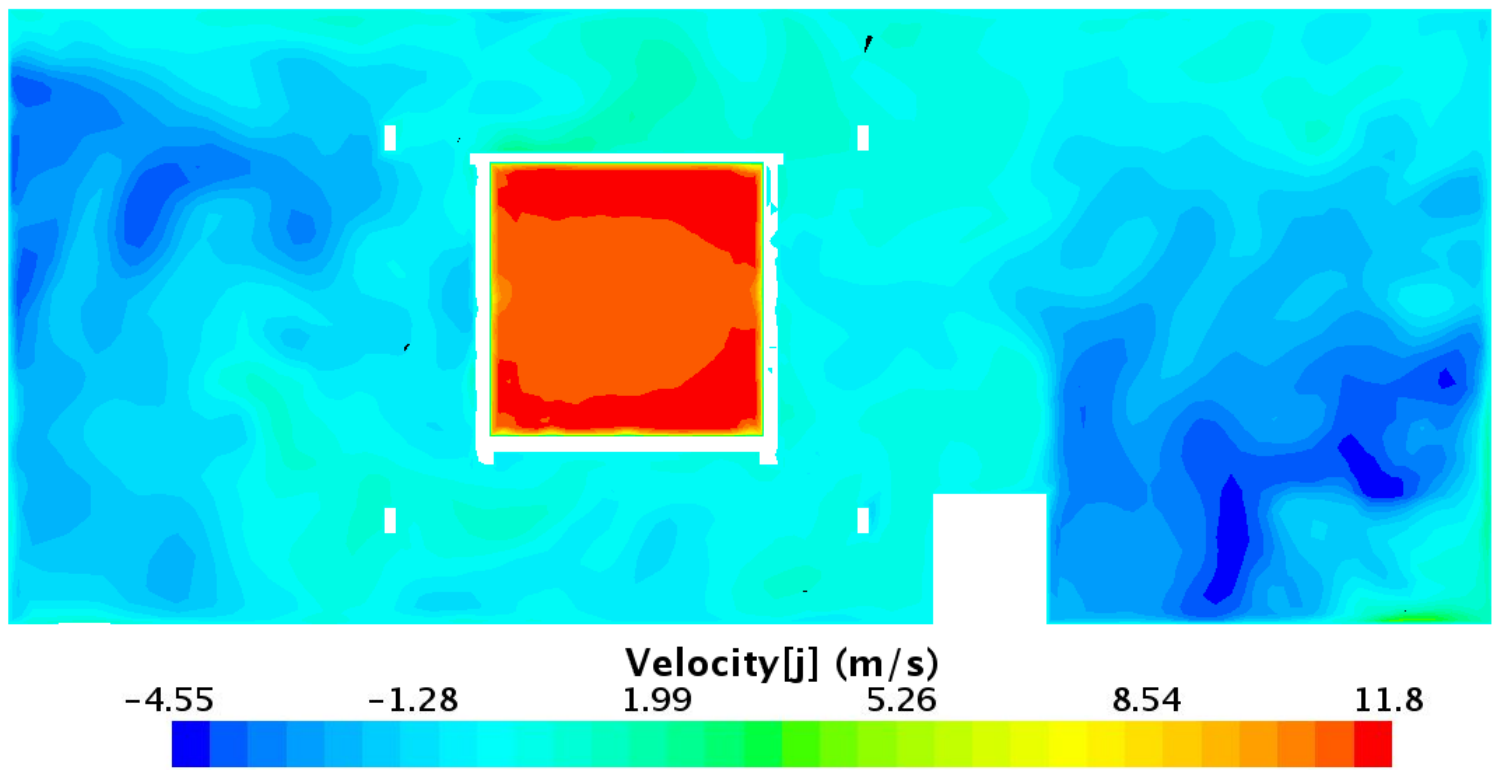

(b) $Y=1.5$ in

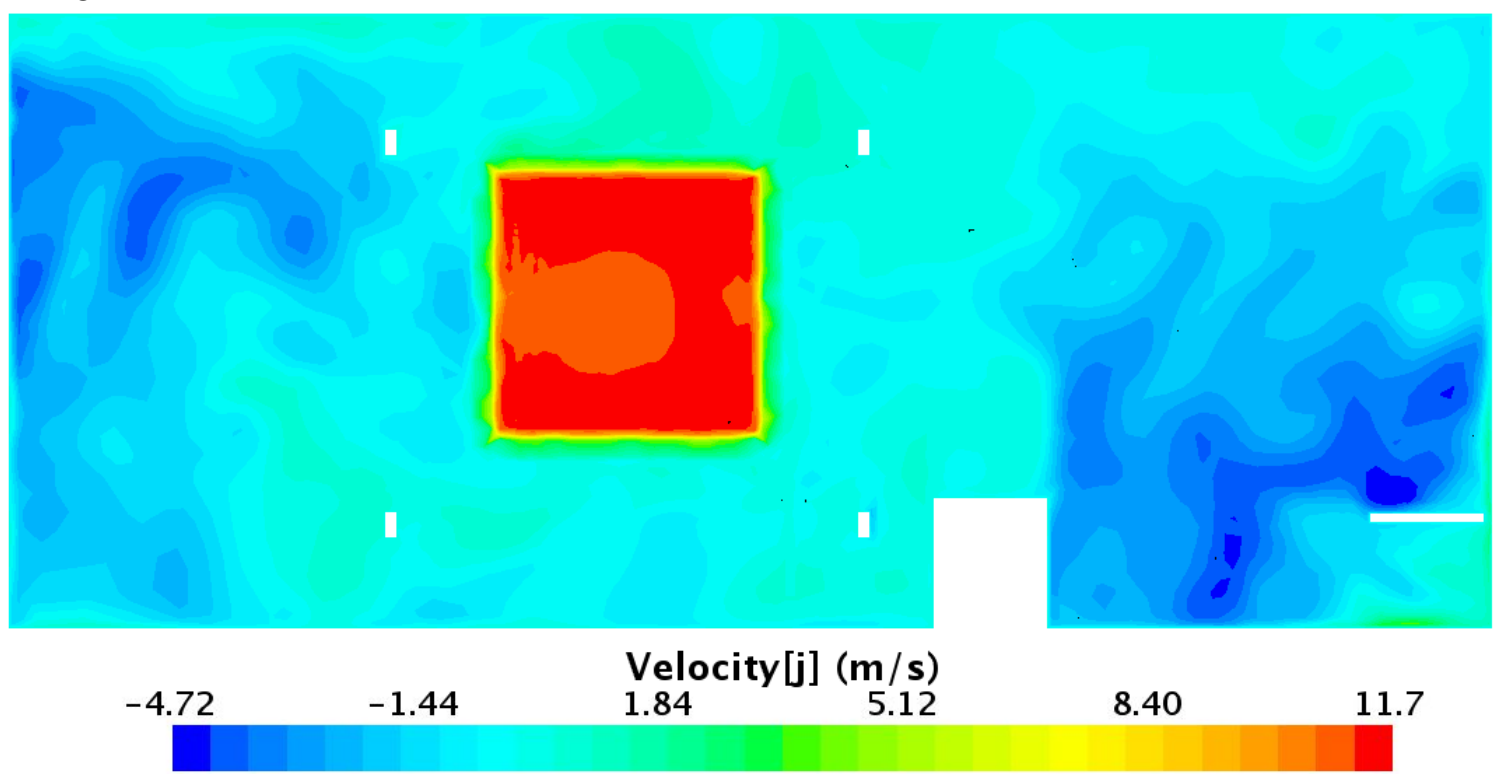


(c) $\mathrm{Y}=1 \mathrm{ft}$
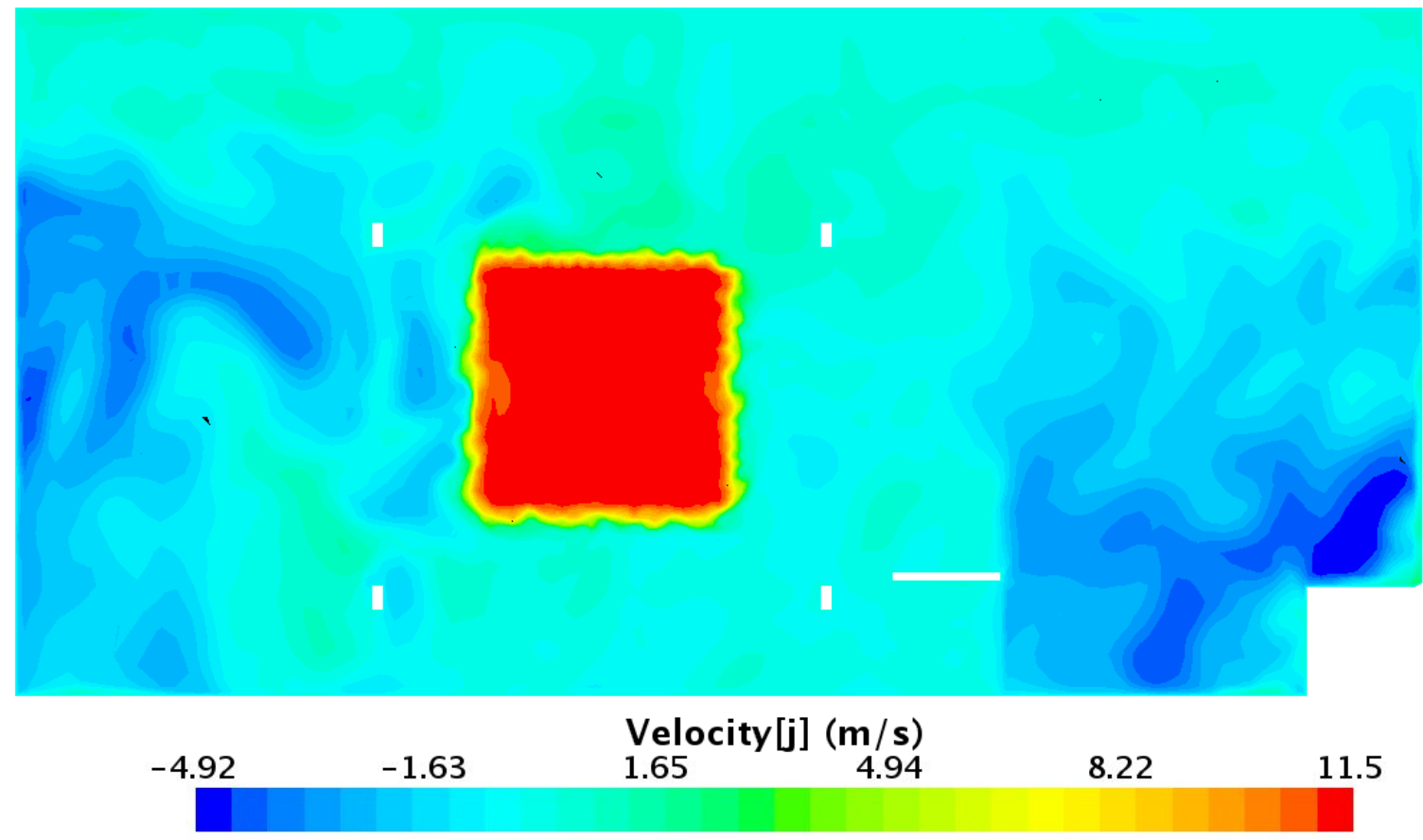

(d) $\quad \mathrm{Y}=2 \mathrm{ft}$
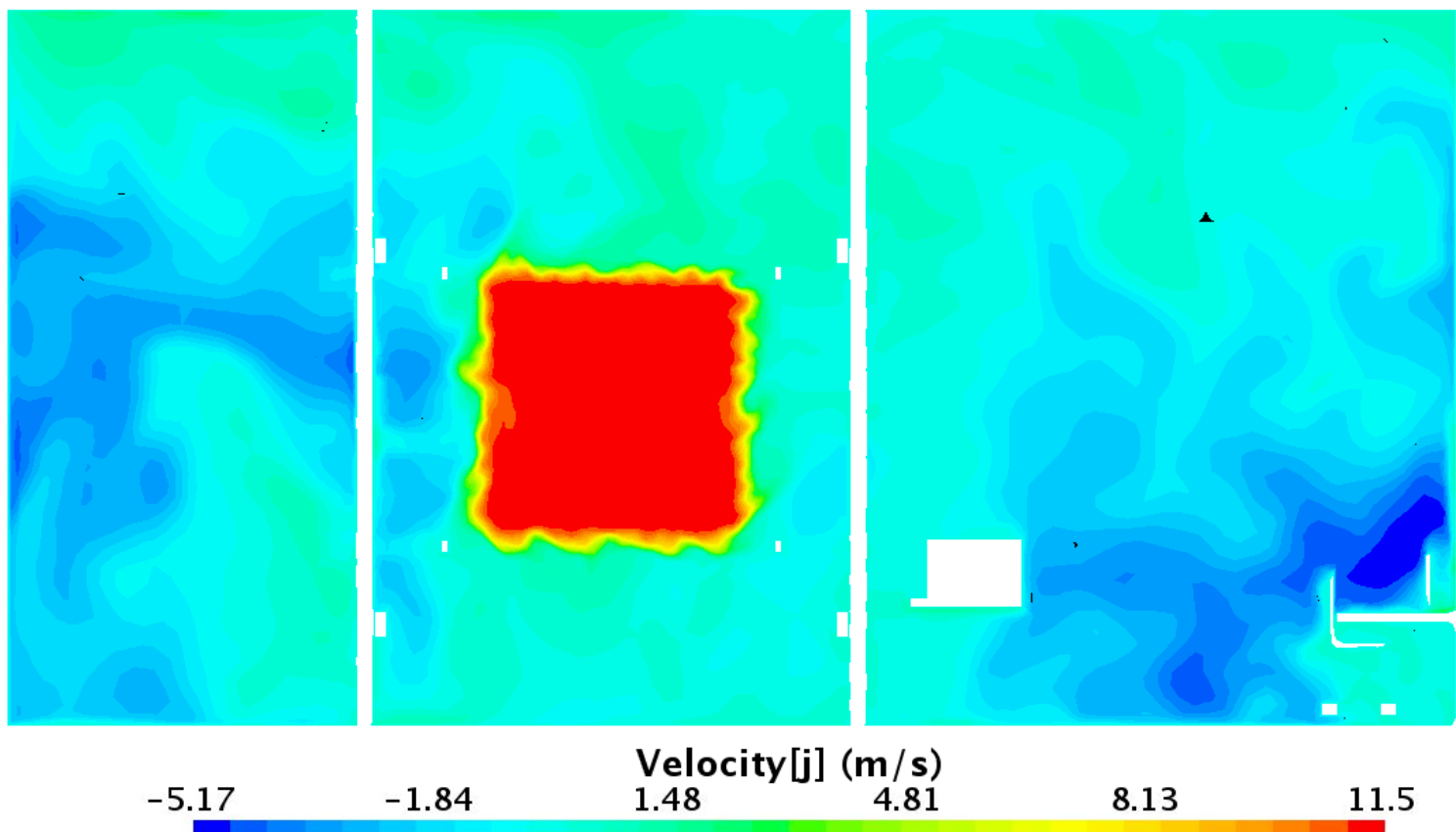

Velocity $[\mathbf{j}](\mathbf{m} / \mathbf{s})$

$-1.84$

4.81

8.13

11.5

Figure 4-23: The distribution of velocity in $Y$ direction on vertical planes (a) $Y=-1.5$ in, (b) $Y=1.5$ in, (c) $\mathrm{Y}=1 \mathrm{ft}$, (d) $\mathrm{Y}=2 \mathrm{ft}$. Model with simplified geometry, LES solver. 


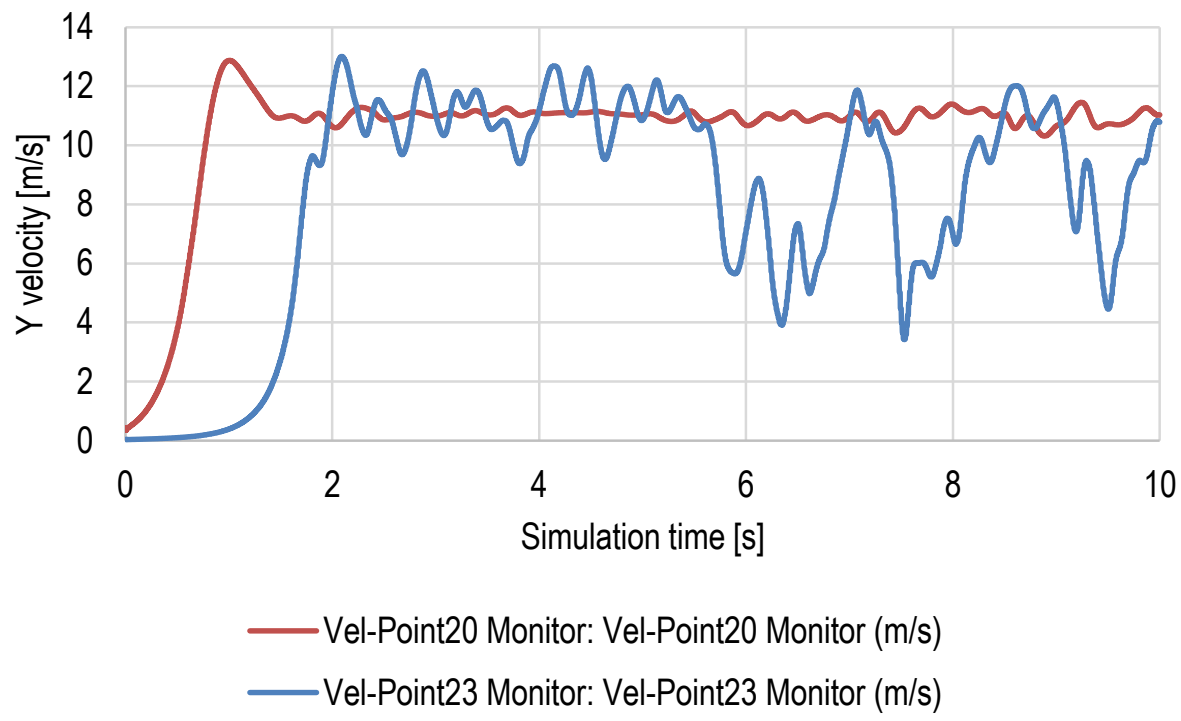

Figure 4-24: Velocity in Y direction at points 20 and 23 vs. simulation time. Model with simplified geometry, LES solver.

\subsection{Other versions of the simplified model - one of the pressure outlet surfaces is closed}

Additional models were created, based on the model with simplified geometry, to assess the influence of the pressure outlet boundary condition surfaces on the air flow. Closing of the left or right hand side outlet did not change the distribution of the air flow significantly, as illustrated in Figure 4-25. The jet did not change shape and velocity magnitudes remain the same, except in the vicinity of the surface that in the previous model was a pressure outlet boundary and now is a wall. The air went around the wind tunnel and exited from the other side. The velocity value at point 20 equaled $10.99 \mathrm{~m} / \mathrm{s}$, and at point 23 it was equal to $11.14 \mathrm{~m} / \mathrm{s}$, in both models.

(a)

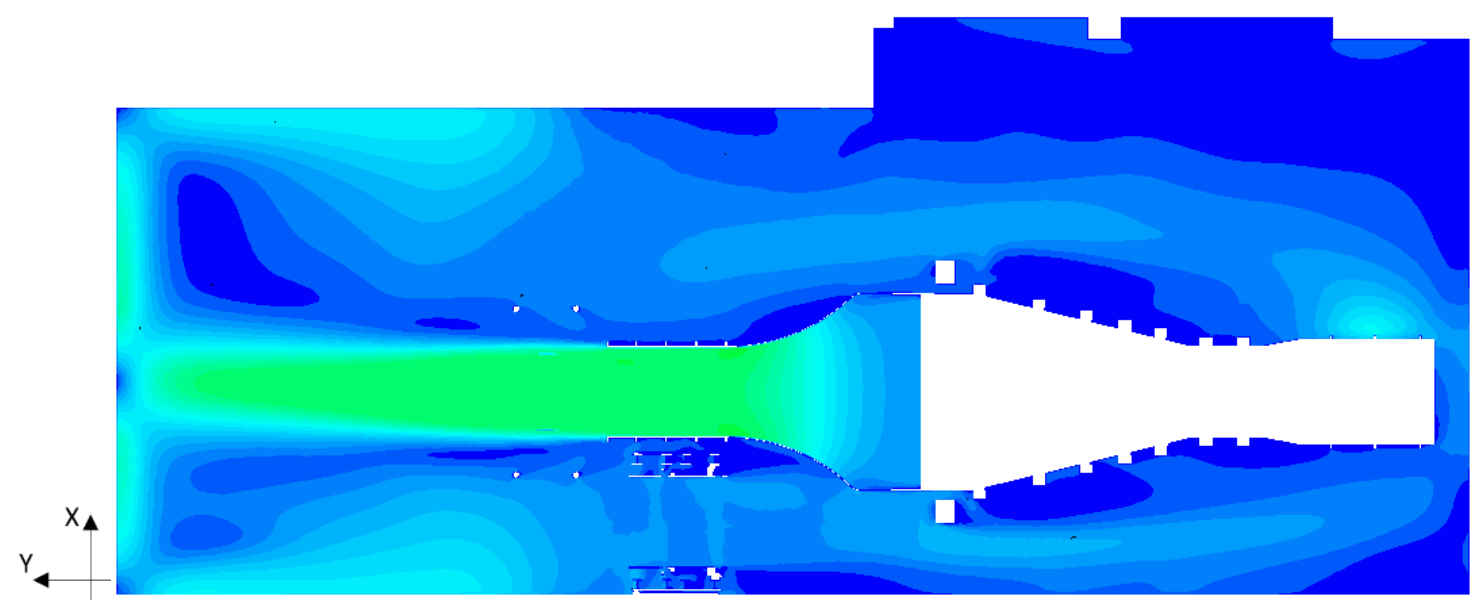


(b)

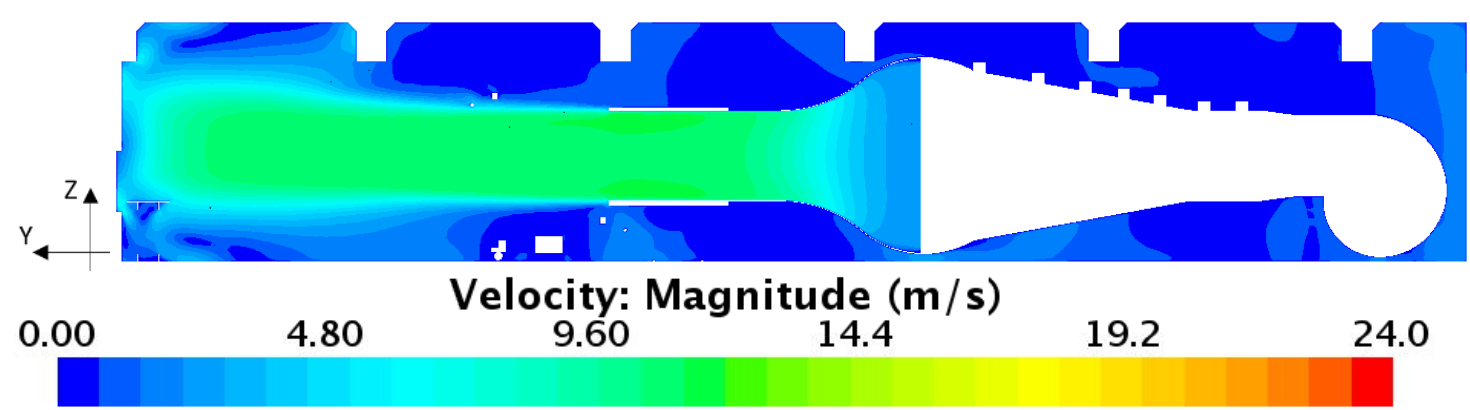

Figure 4-25: Velocity magnitude distribution (a) on a horizontal plane, (b) on a vertical plane, in the model with left hand side pressure outlet blocked

(a)

(b)

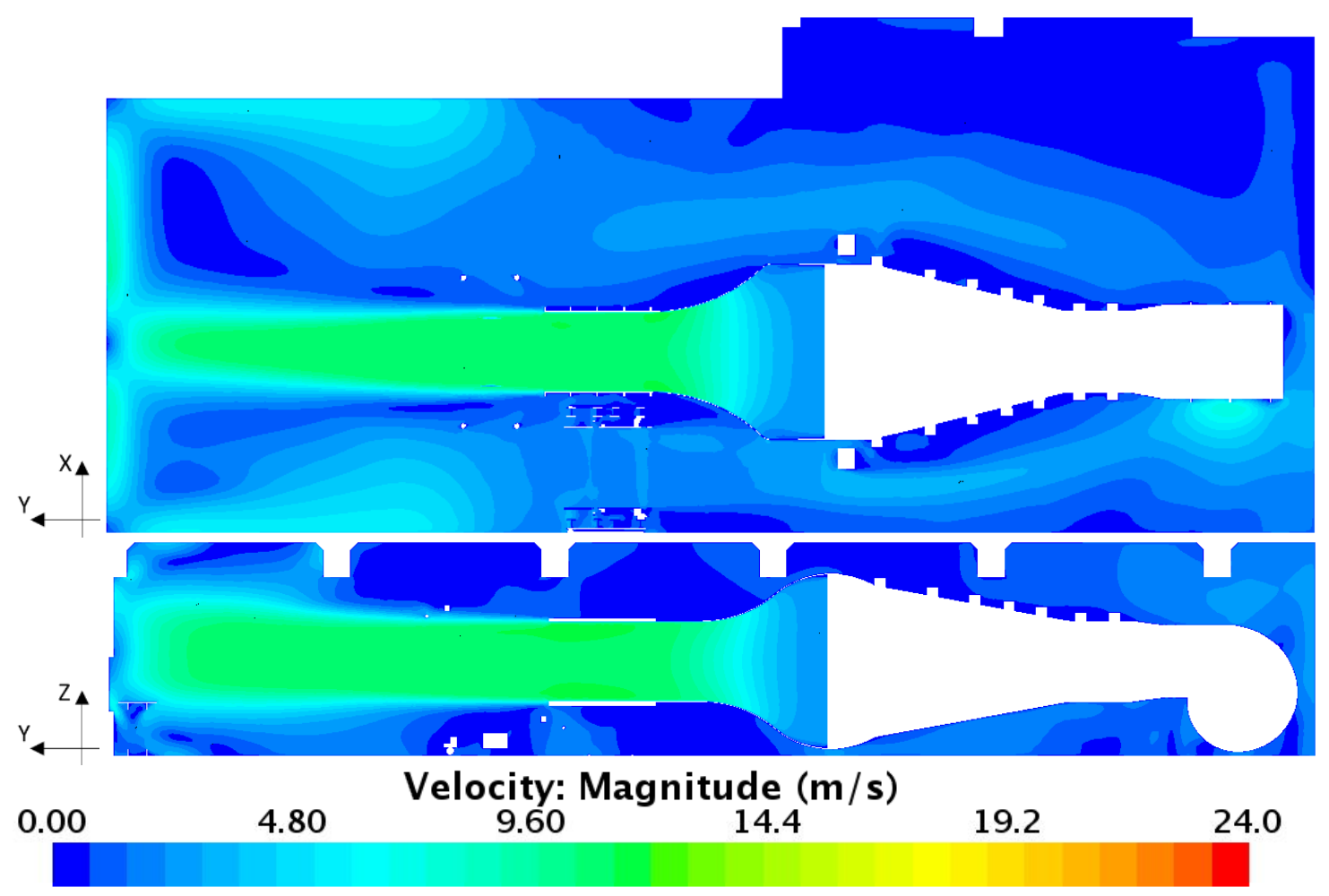

Figure 4-26: Velocity magnitude distribution (a) on a horizontal plane, (b) on a vertical plane, in the model with right hand side pressure outlet blocked

\subsection{Other versions of the simplified model - non-uniform inlet parameter distributions}

A velocity inlet type of boundary condition was applied, as in the previously described simplified models. The difference is that the applied turbulence intensity, turbulent viscosity ratio, and velocity components were introduced as a distribution over the inlet surface, using time averaged

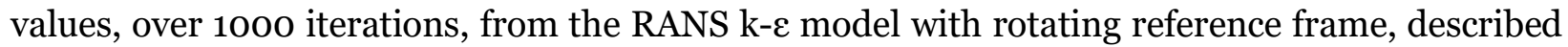


in Chapter 4.3.1. In the model described in Chapter 4.3.1 the inlet velocity was constant, with the value calculated based on fan speed from the relation in Figure 4-9, turbulence intensity was set to the default value of 0.01 , and turbulent viscosity ratio was set to the default value of 10 . In the model presented in this chapter, the surface distribution of the inlet parameters is defined in a table, imported from a separate Star-CCM+ simulation file. The comparison between the models is performed at two fan speeds, $48 \mathrm{rpm}$ and $437 \mathrm{rpm}$, which are respectively the lowest and the highest out of those analyzed in the laboratory. The averaging was done over 1000 iterations, after the solution converged. The values of interest at the volume cell nodes on the last screen were saved every 10 iterations in a *.csv file. An external Python script was created which would calculate time averaged values at points and create an input file in the right format for the simplified model. Figure 4-27 shows the inlet velocity boundary condition input parameters at fan speed 48 rpm: (a) X velocity, (b) Y velocity, (c) Z velocity, (d) turbulence intensity, (e) turbulent viscosity ratio mapped onto the inlet boundary surface.

(a)
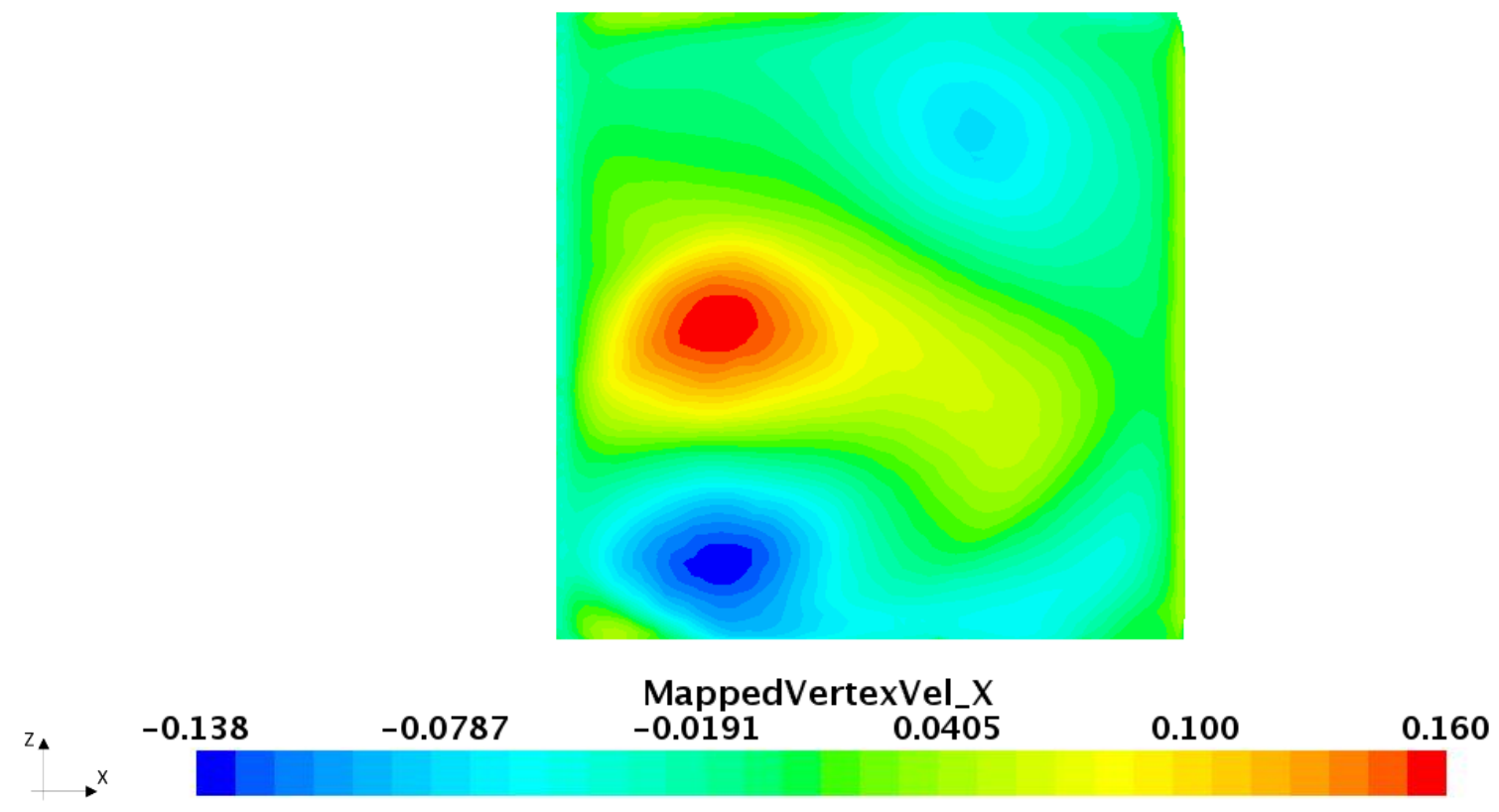
(b)
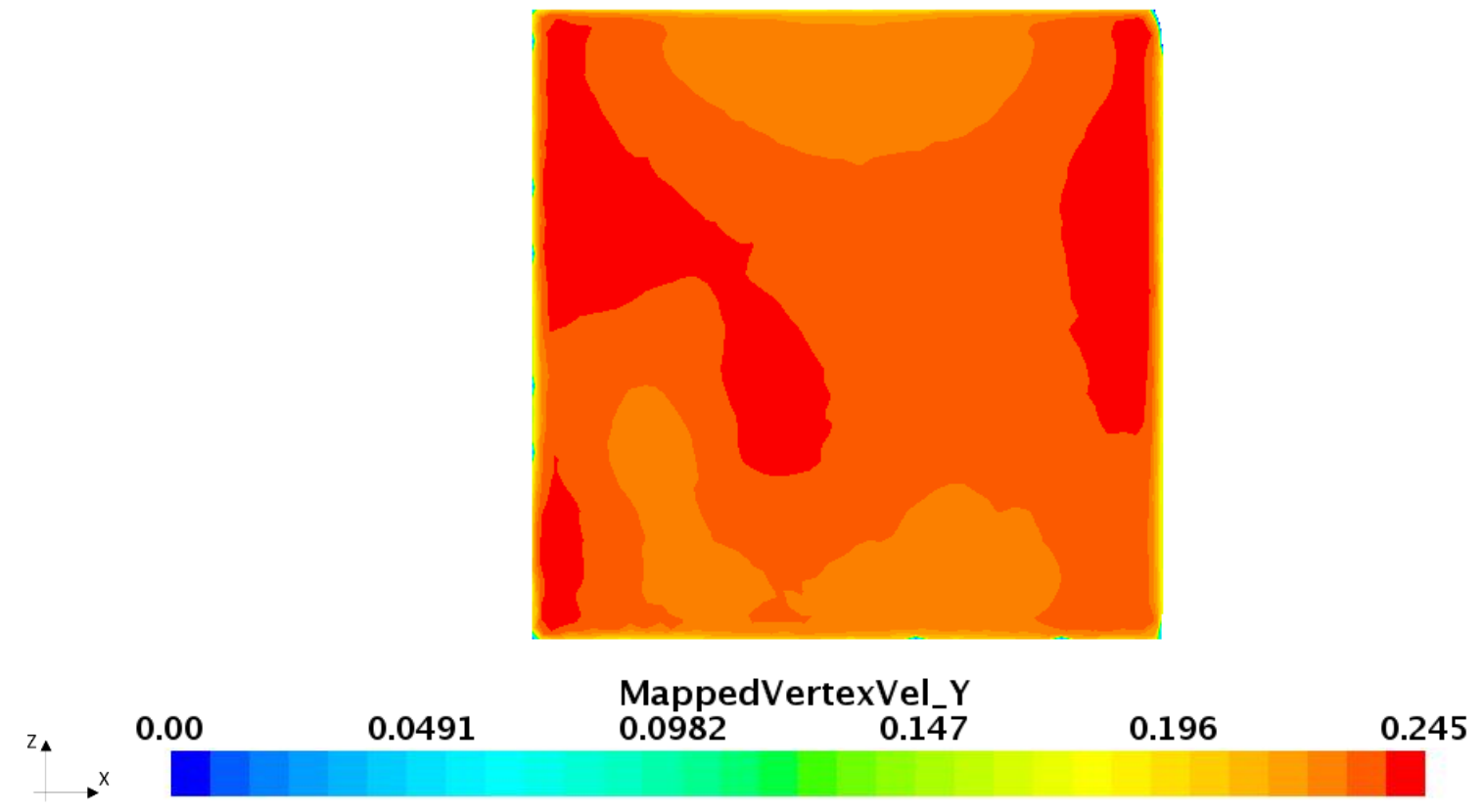

(c)
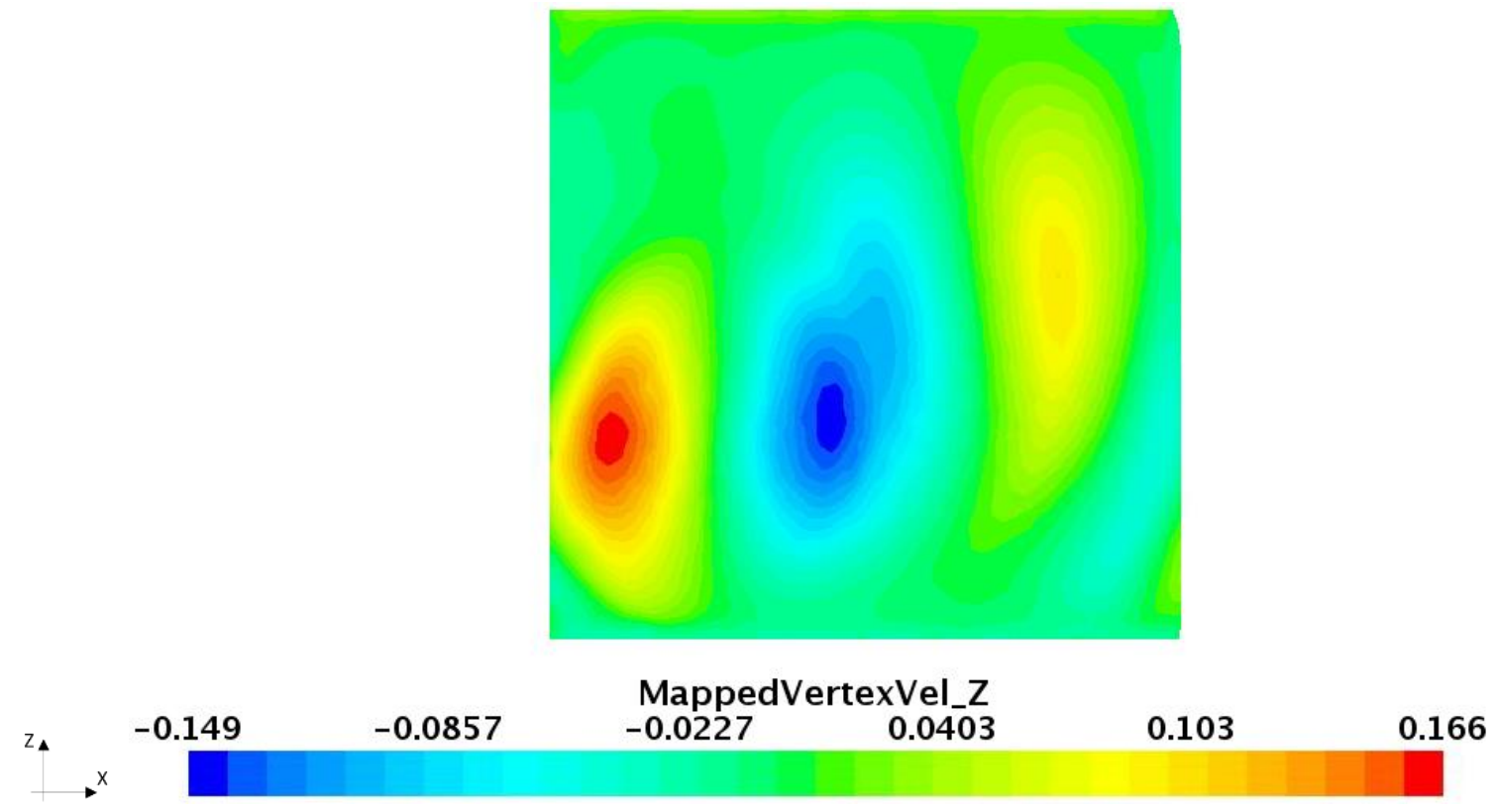
(d)
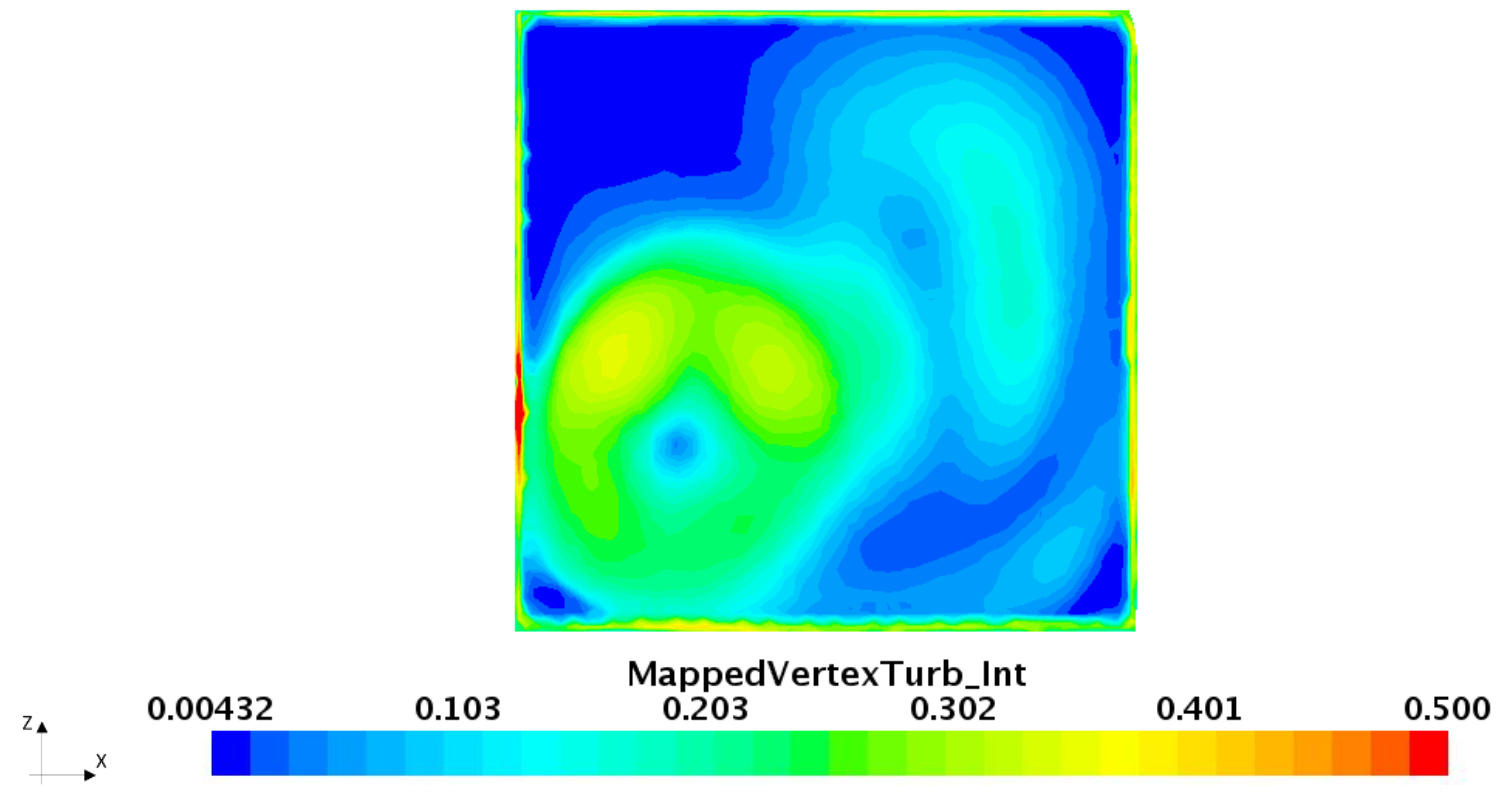

(e)

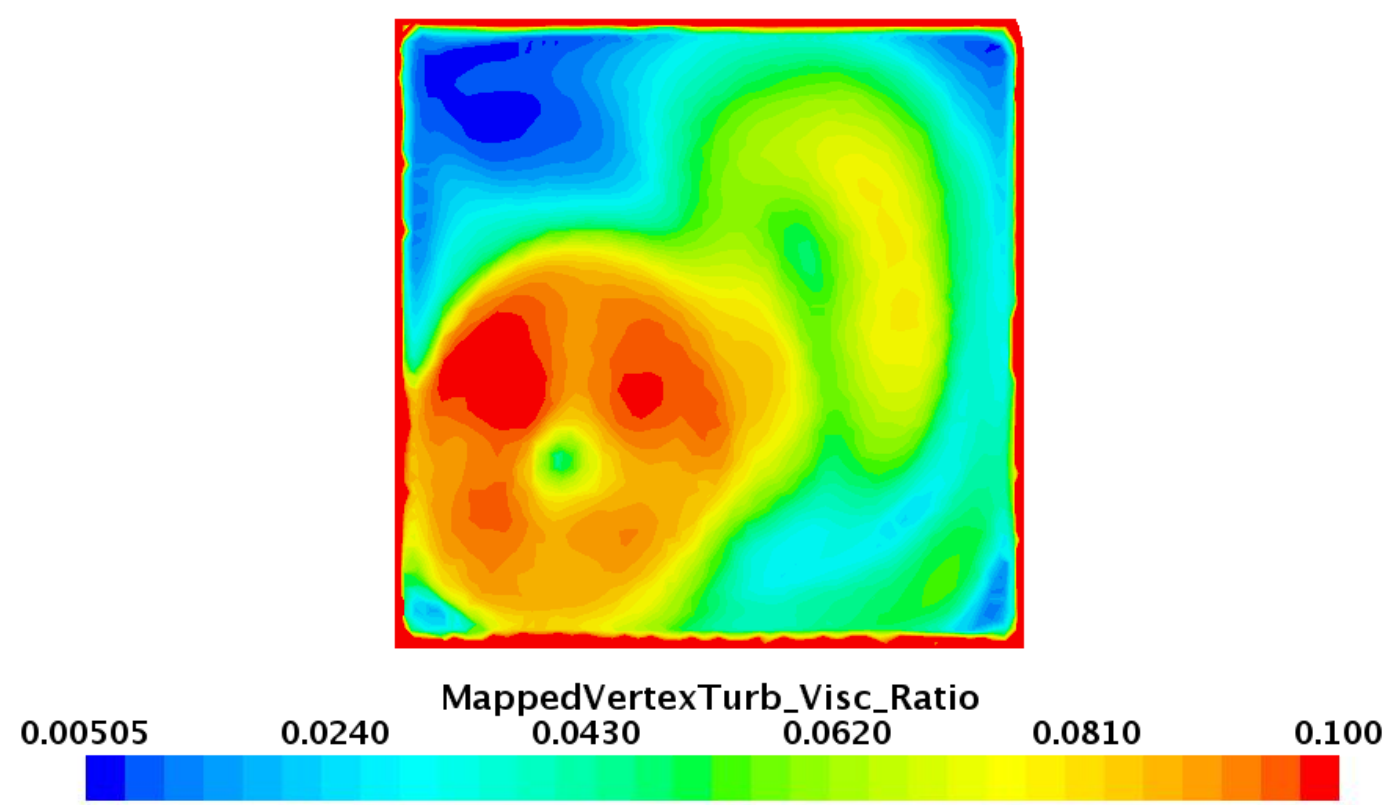

Figure 4-27: Inlet velocity boundary condition input parameters at fan speed $48 \mathrm{rpm}$ : (a) X velocity, (b) Y velocity, (c) $\mathrm{Z}$ velocity, (d) turbulence intensity, (e) turbulent viscosity ratio 
(a)

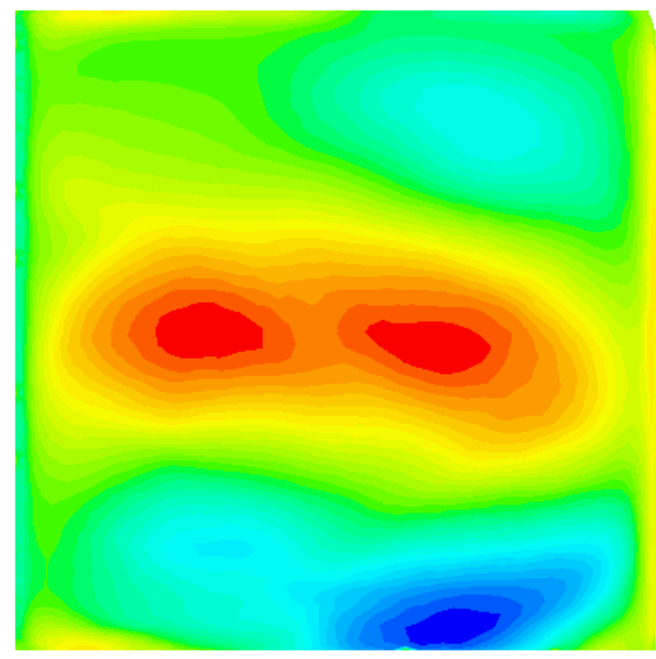

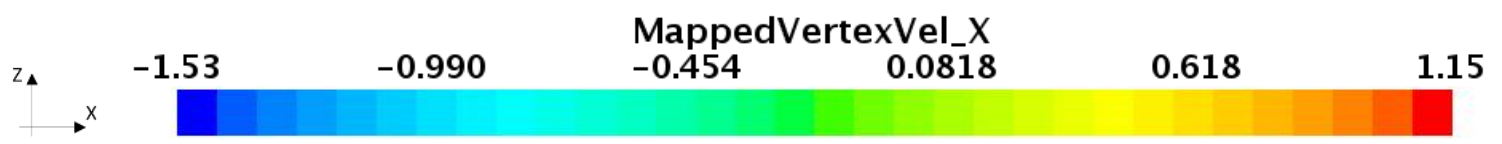

(b)

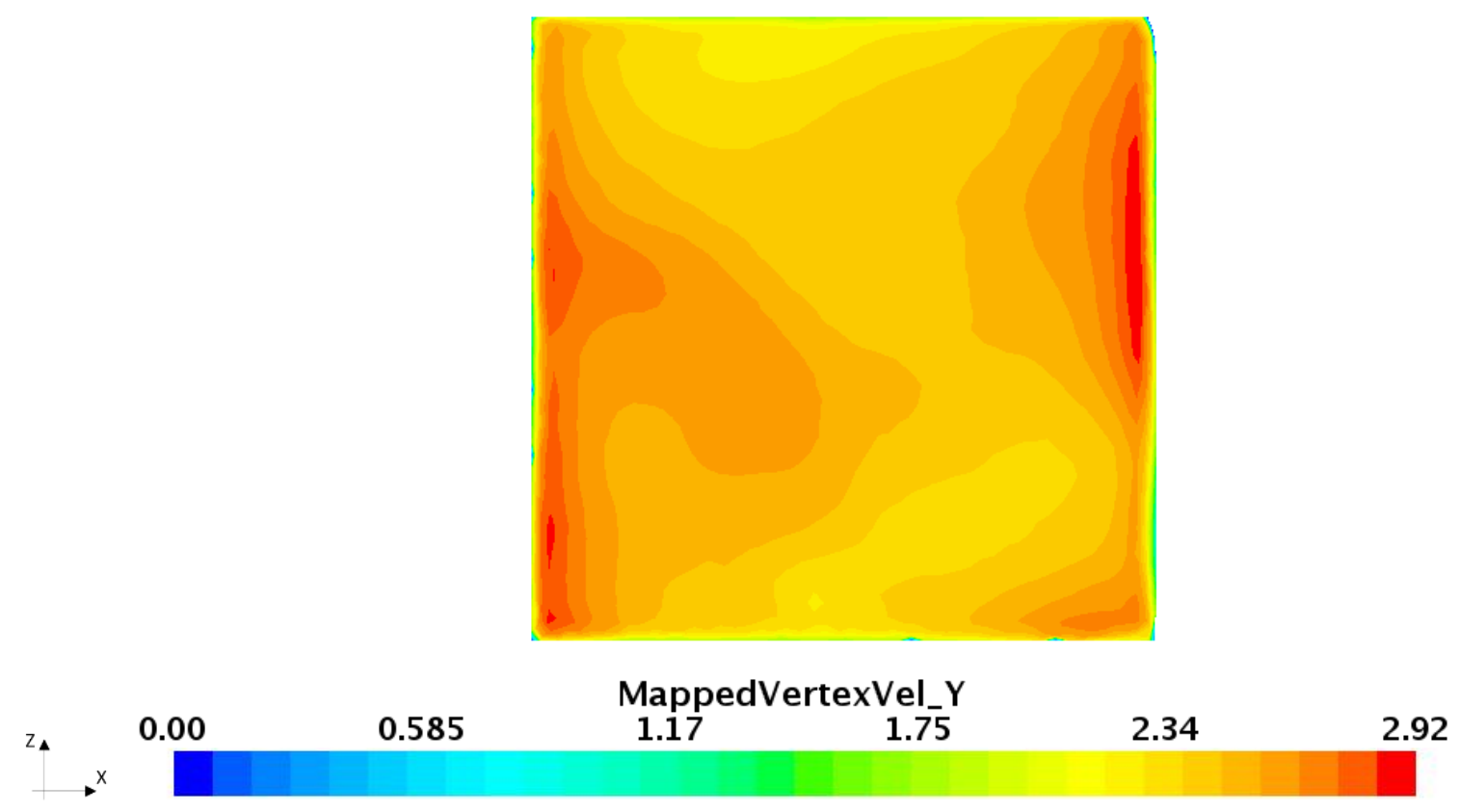


(c)
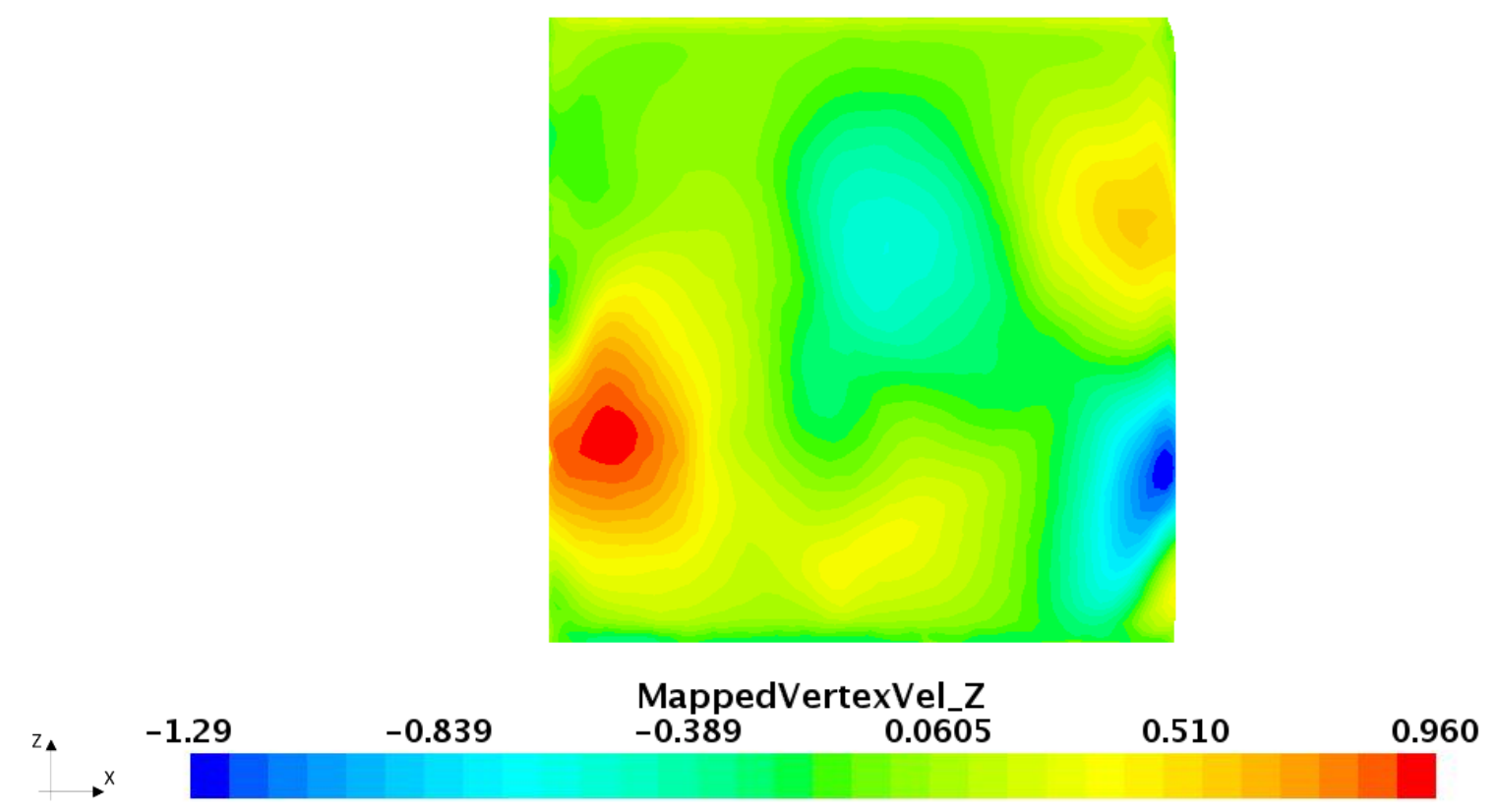

(d)
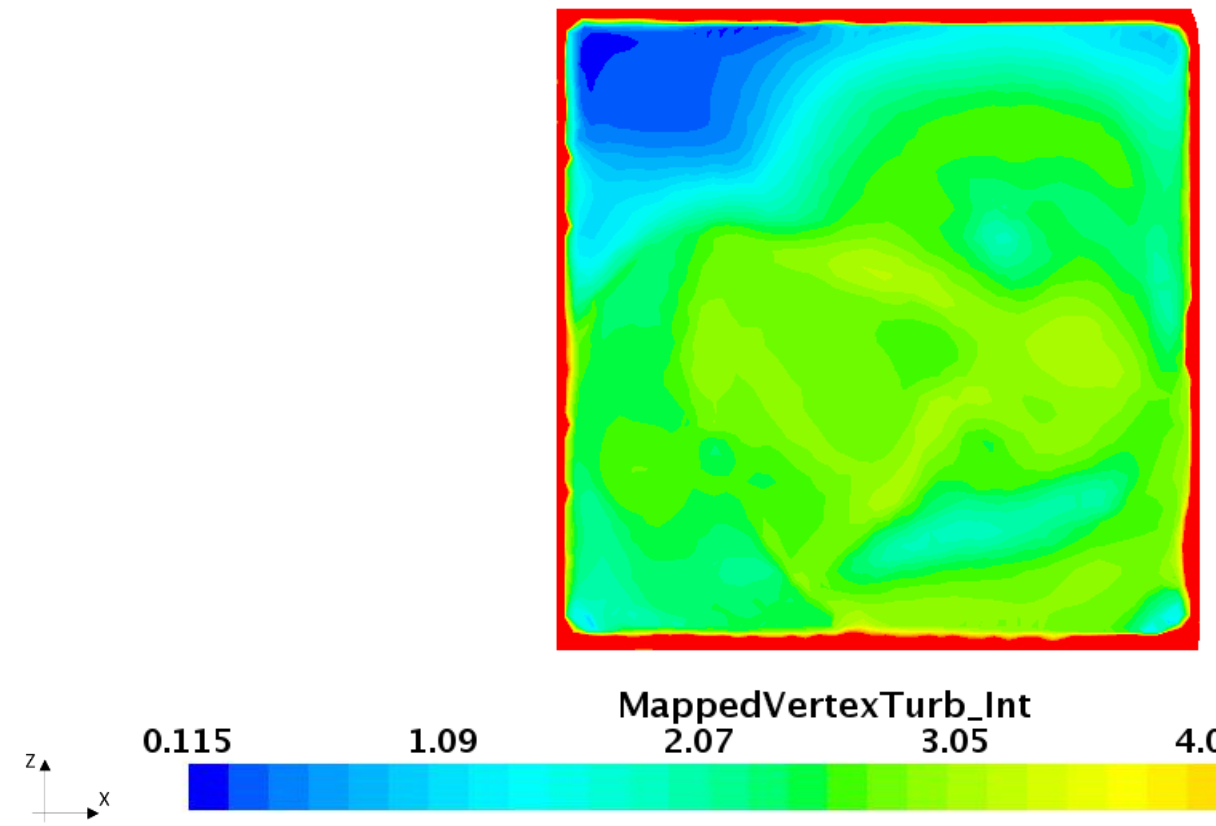

MappedVertexTurb_Int

1.09

2.07

3.05

4.02

5.00 
(e)
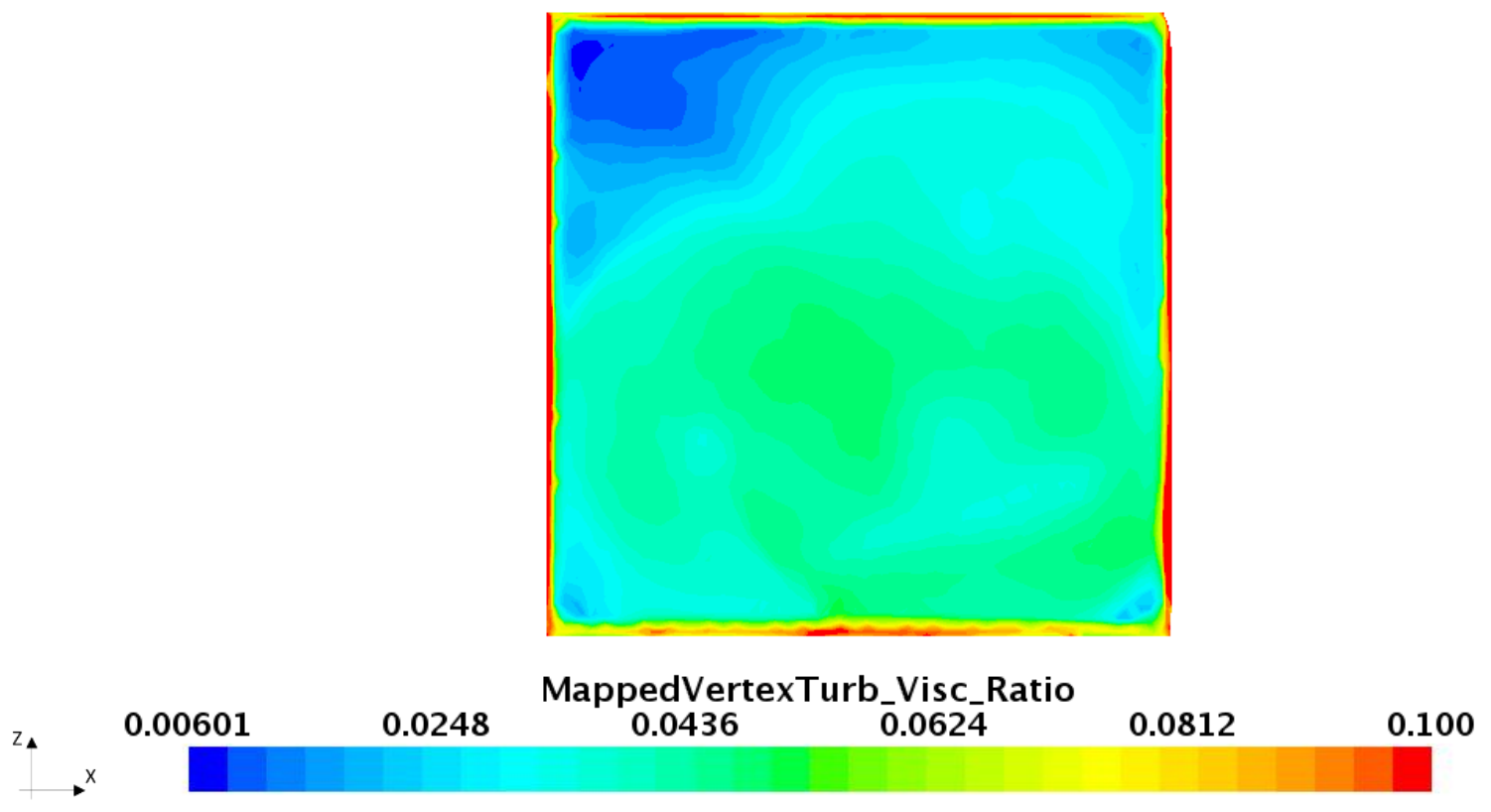

Figure 4-28: Inlet velocity boundary condition input parameters at fan speed $437 \mathrm{rpm}$ : (a) X velocity, (b) Y velocity, (c) Z velocity, (d) turbulence intensity, (e) turbulent viscosity ratio

Table 4-5: Average velocity magnitude at points 20 and 23

\begin{tabular}{|c|c|c|c|c|}
\hline & \multicolumn{2}{|c|}{ 48 rpm } & \multicolumn{2}{|c|}{ 437 rpm } \\
\hline $\begin{array}{c}\text { Type of CFD } \\
\text { model }\end{array}$ & $\begin{array}{c}\text { Velocity } \\
\text { magnitude at } \\
\text { point 20 } \\
{[\mathbf{m} / \mathbf{s}]}\end{array}$ & $\begin{array}{c}\text { Velocity } \\
\text { magnitude at } \\
\text { point 23 [m/s] }\end{array}$ & $\begin{array}{c}\text { Velocity } \\
\text { magnitude at } \\
\text { point 20 } \\
{[\mathbf{m} / \mathbf{s}]}\end{array}$ & $\begin{array}{c}\text { Velocity } \\
\text { magnitude at } \\
\text { point 23 [m/s] }\end{array}$ \\
\hline Experiment & - & - & 10.50 & 9.81 \\
\hline $\begin{array}{c}\text { Rotating } \\
\text { reference } \\
\text { frame }\end{array}$ & 1.04 & 0.80 & 11.07 & 7.65 \\
\hline $\begin{array}{c}\text { Simplified } \\
\text { geometry with } \\
\text { uniform inlet } \\
\text { velocity }\end{array}$ & 1.01 & 1.00 & 10.71 & 10.65 \\
\hline $\begin{array}{c}\text { Simplified } \\
\text { geometry with } \\
\text { non-uniform } \\
\text { inlet velocity }\end{array}$ & 1.05 & 0.99 & 10.95 & 10.05 \\
\hline
\end{tabular}

The average velocity magnitudes at points 20 and 23 obtained from three models are presented in Table 4-5. The compared models are: the rotating reference frame model, presented in chapter 4.2.1, the model with simplified geometry and uniform parameters at the inlet boundary condition, presented in chapter 4.3.1, and the model presented here. Also, experimental measurements are included for the $437 \mathrm{rpm}$ fan speed. The results for the $48 \mathrm{rpm}$ speed are not available. The comparison shows that the velocities at the two selected points are very close to 
each other for the simplified models. They are in good agreement for all three models at point 20, which is closer to the testing area. The jet in the models with simplified geometry is uniform nearly up to the front wall, and in the RRF model it slows down, as was stated before. Therefore, at point 23, which is closer to the wall, there is a difference in the results between the models. The resulting velocity magnitude distribution, at fan speed $48 \mathrm{rpm}$, on a vertical plane is presented in Figure 4-29 and on a horizontal plane in Figure 4-30. The corresponding plots for fan speed $437 \mathrm{rpm}$ are shown in Figure 4-31 and Figure 4-32.

Appendix B contains a detailed presentation of the velocity and turbulence variation at points on a vertical plane, located in the test section, $60 \mathrm{~cm}$ away from the wind tunnel extension, at fan speed $48 \mathrm{rpm}$ and $437 \mathrm{rpm}$.

(a)
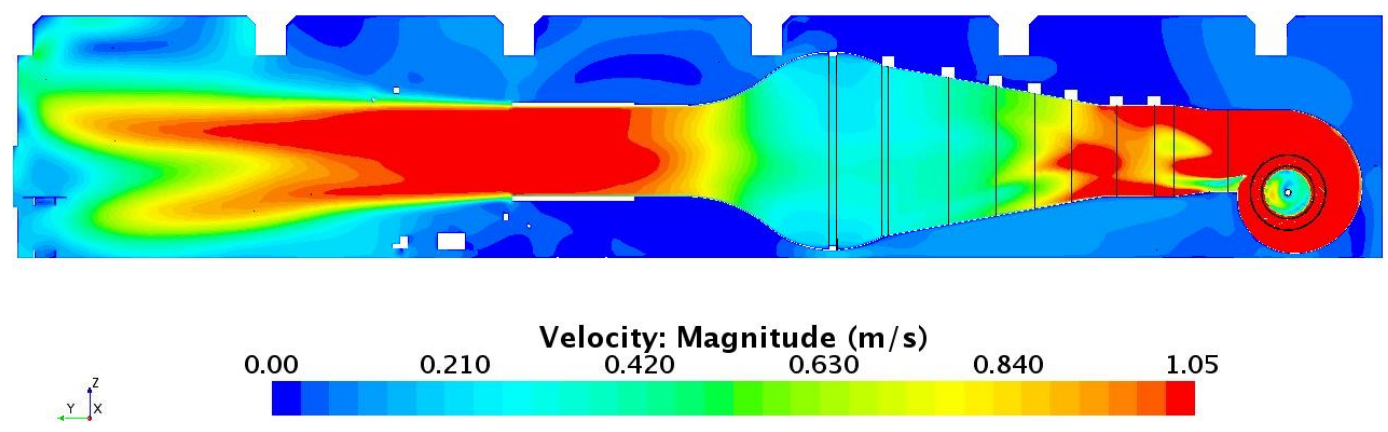

(b)
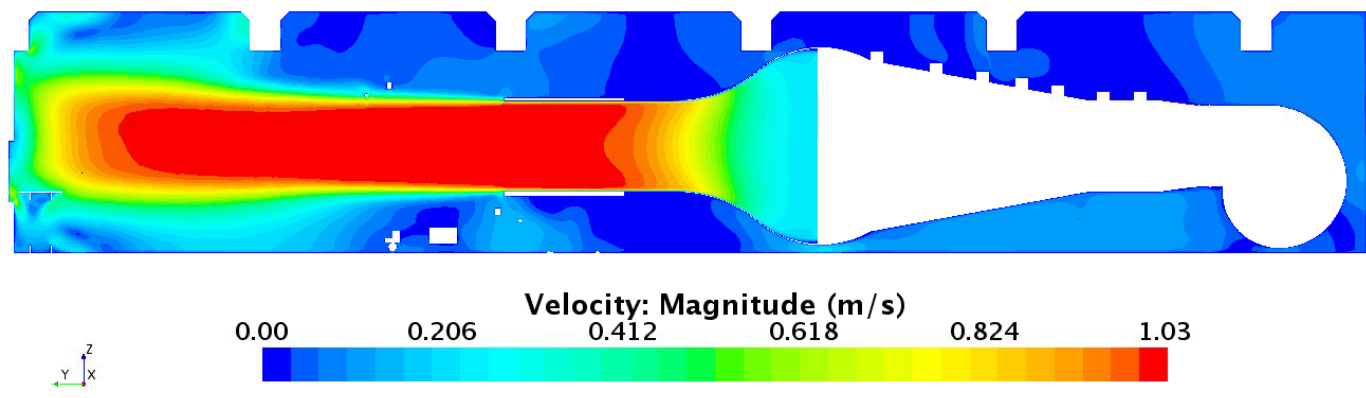

(c)

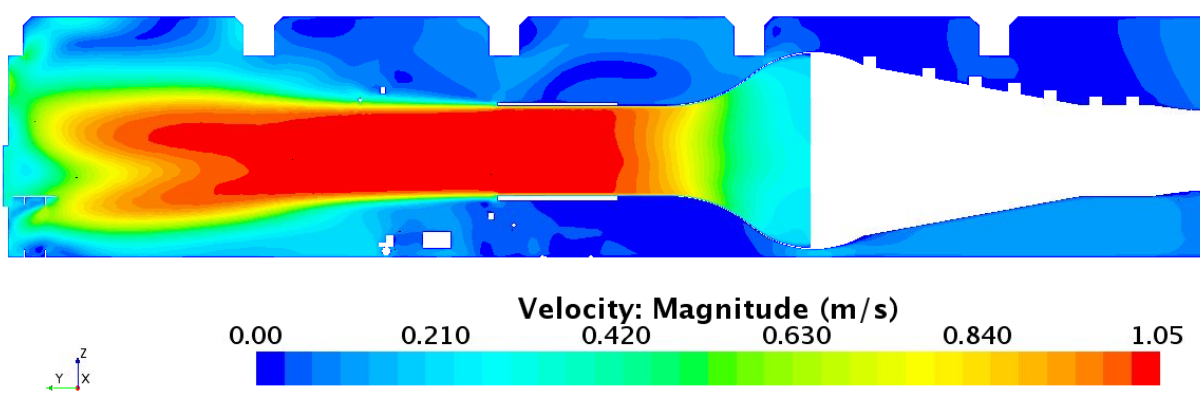

Figure 4-29: Side view of the velocity magnitude distribution at fan speed $48 \mathrm{rpm}$ on a vertical plane for models: (a) with rotating reference frame, (b) simplified geometry with uniform inlet velocity, (c) simplified geometry with non-uniform inlet velocity 
(a)

(b)

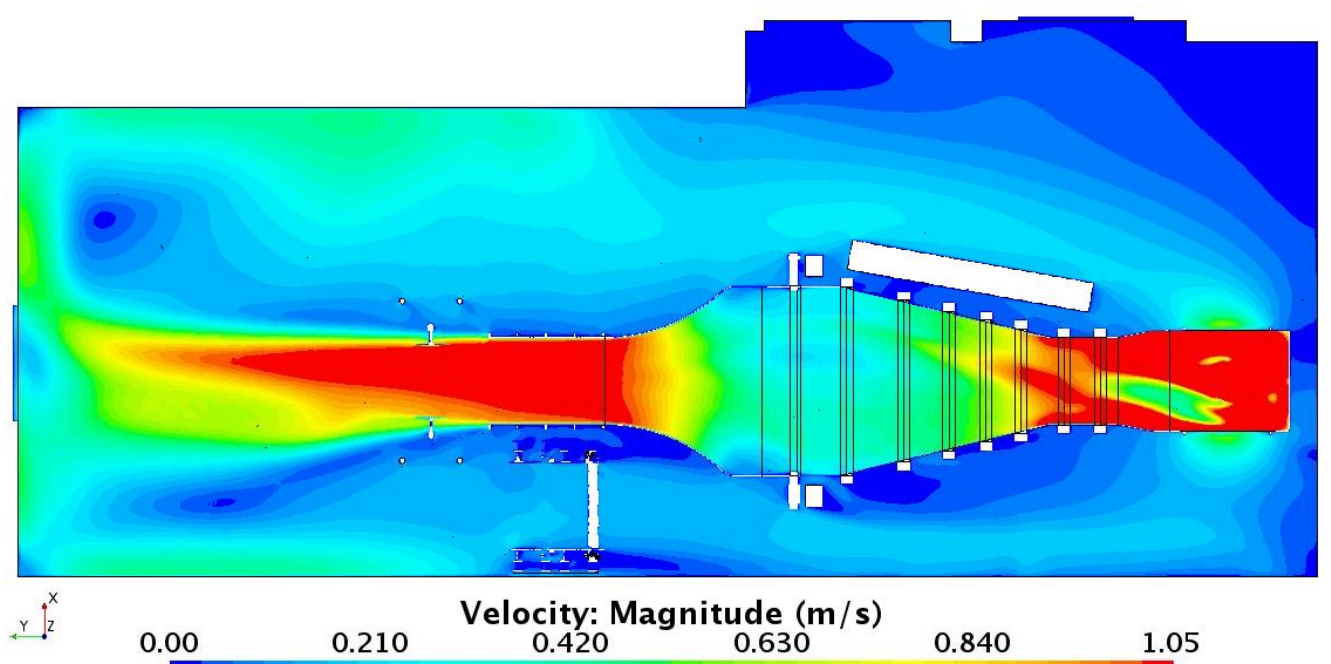

(c)

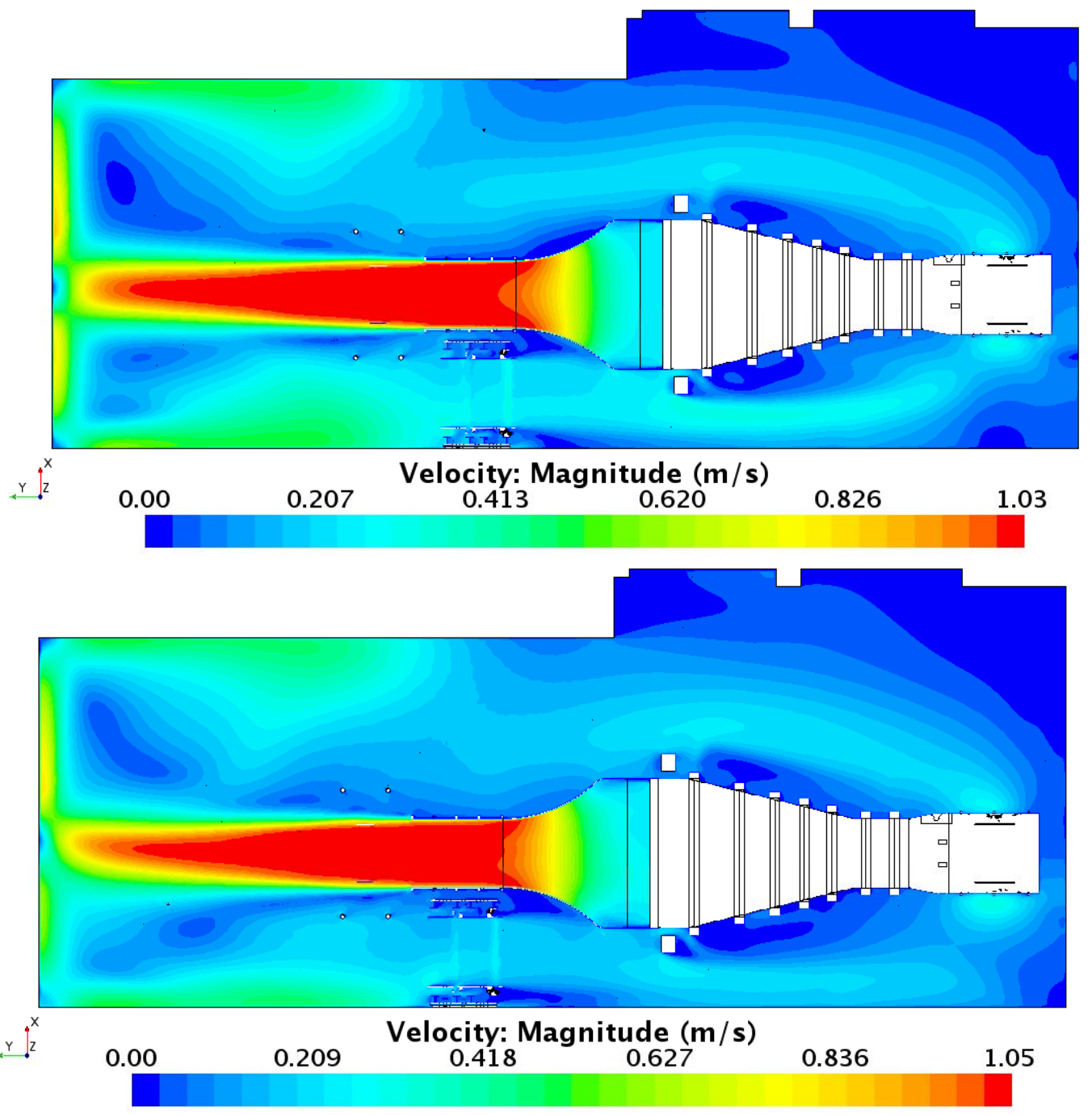

Figure 4-30: Top view of the velocity magnitude distribution at fan speed $48 \mathrm{rpm}$ on a horizontal plane for models: (a) with rotating reference frame, (b) simplified geometry with uniform inlet velocity, (c) simplified geometry with non-uniform inlet velocity 
(a)
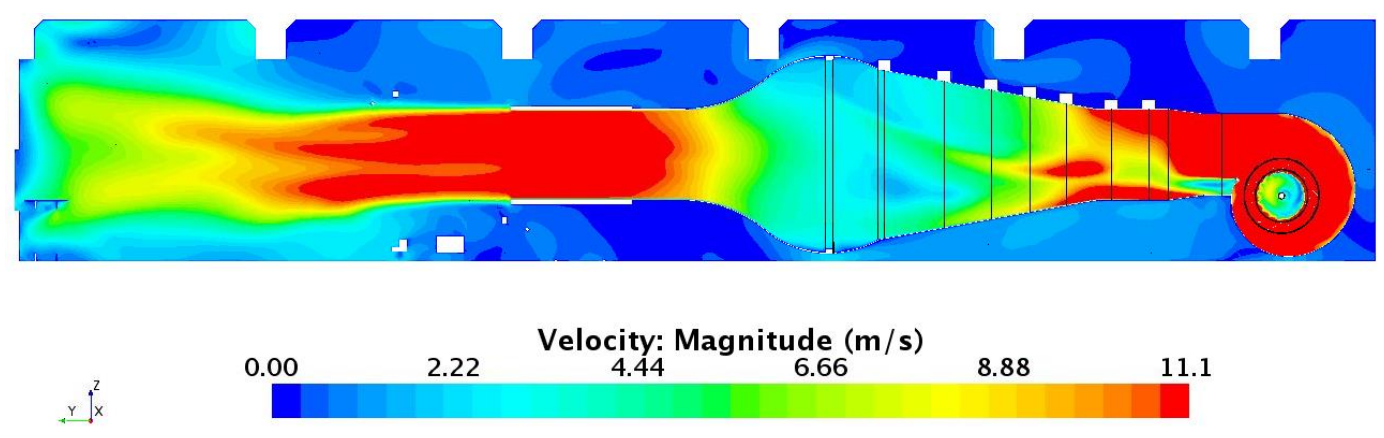

(b)
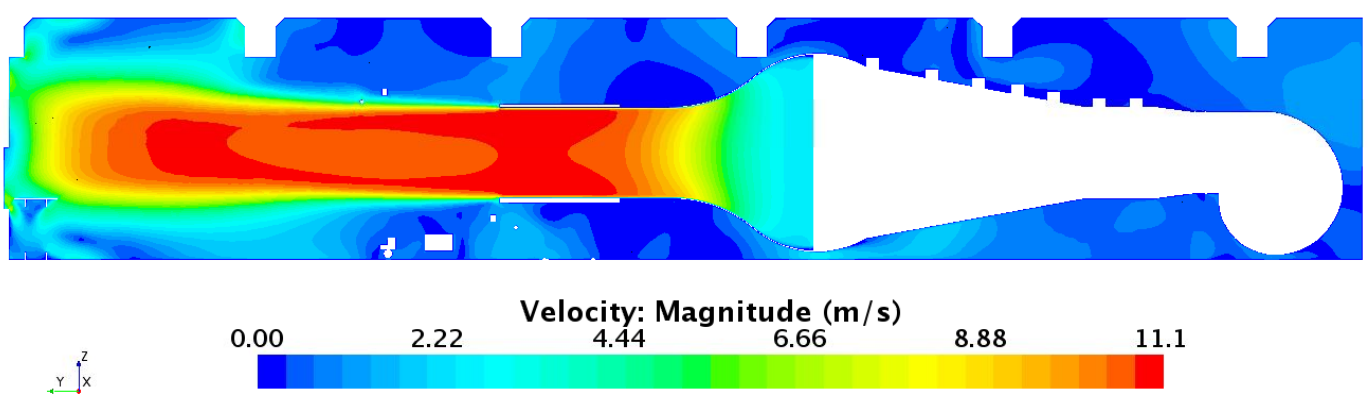

Velocity: Magnitude $(\mathrm{m} / \mathrm{s})$ 6.66

8.88

11.1

(c)

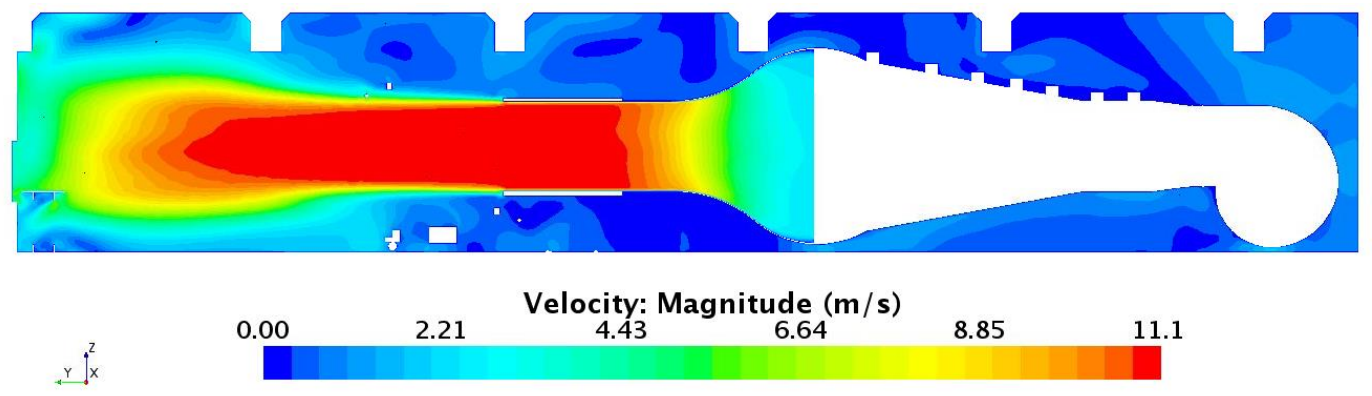

Figure 4-31: Side view of the velocity magnitude distribution at fan speed $437 \mathrm{rpm}$ on a vertical plane for models: (a) with rotating reference frame, (b) simplified geometry with uniform inlet velocity, (c) simplified geometry with non-uniform inlet velocity 
(a)

(b)
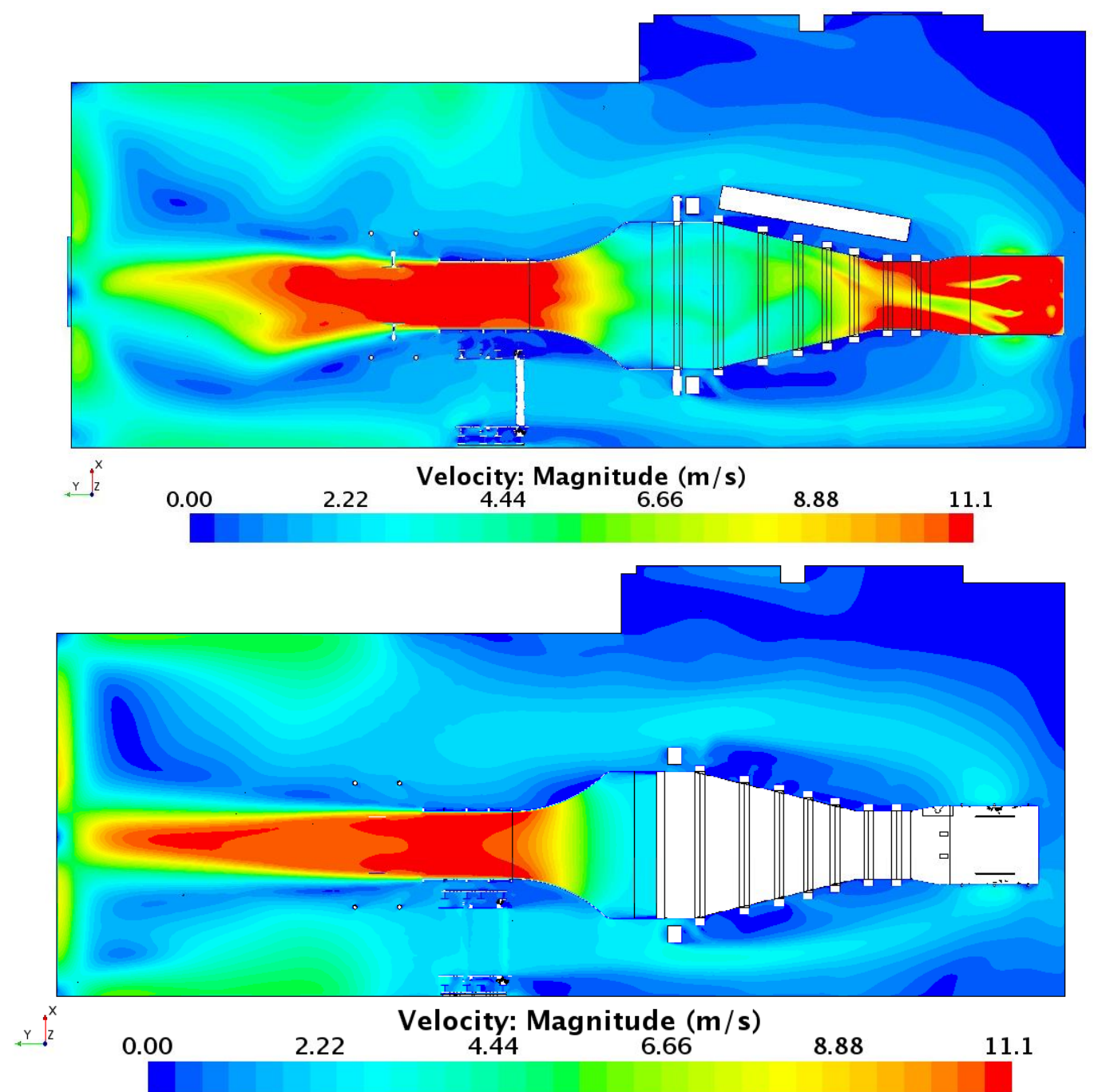

(c)

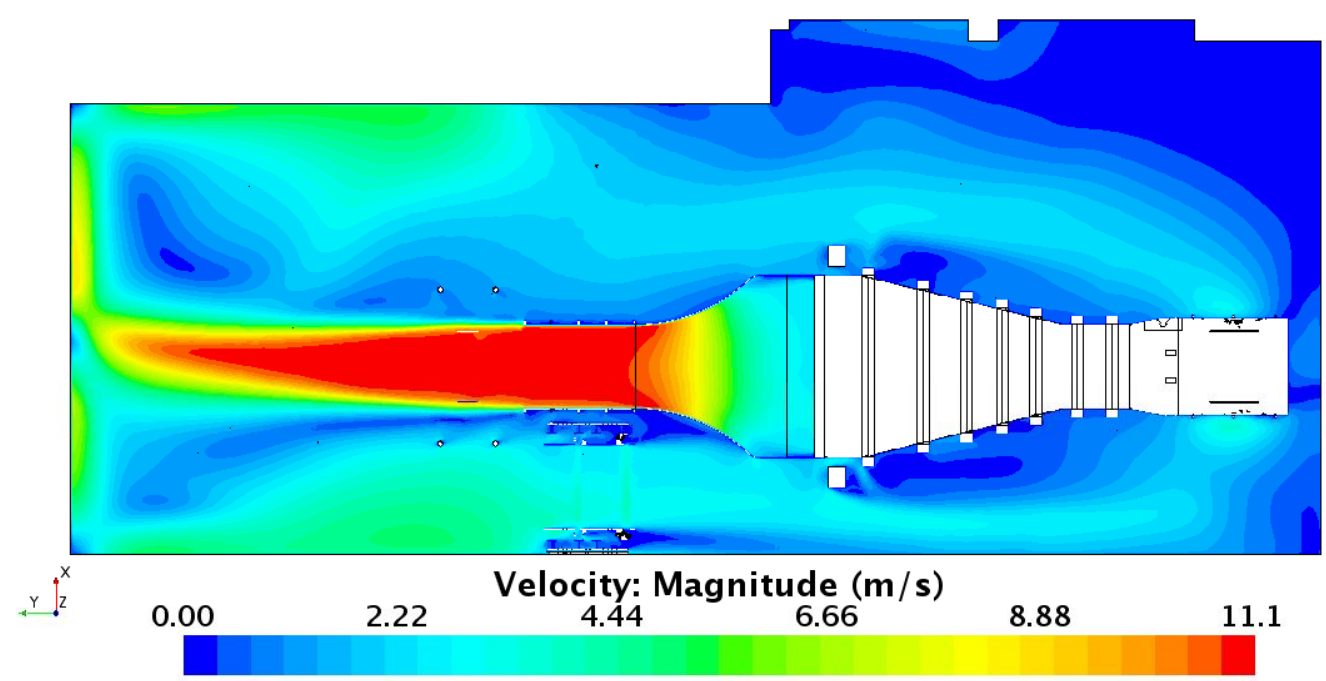

Figure 4-32: Top view of the velocity magnitude distribution at fan speed $437 \mathrm{rpm}$ on a horizontal plane for models: (a) with rotating reference frame, (b) simplified geometry with uniform inlet velocity, (c) simplified geometry with non-uniform inlet velocity 


\subsection{Other versions of the simplified model - back half of the room is cut off}

In an attempt to further simplify the domain, it was decided to crop the back part of the room and analyze the air flow in the new setup. As shown in chapter 4.4, the flow in the room does not change significantly when the left or right hand side outlet is closed. In the new model, the number of cells is reduced by $30 \%$ compared to the simplified model. Nevertheless, this model converges slower than the one described in chapter 4.3. The simulation needed to be run for at least 5000 iterations to get convergence, which took about 3 hours, see Figure 4-33. Moreover, the velocity values were higher than expected. At point 20 it reaches $11.9 \mathrm{~m} / \mathrm{s}$, and at point 23 it is $12.04 \mathrm{~m} / \mathrm{s}$. The velocity magnitude distribution in the domain is shown in Figure 4-34. Since this configuration does not save significant computational resources or time or produce results as accurate as the other simplified model, it is not recommended.

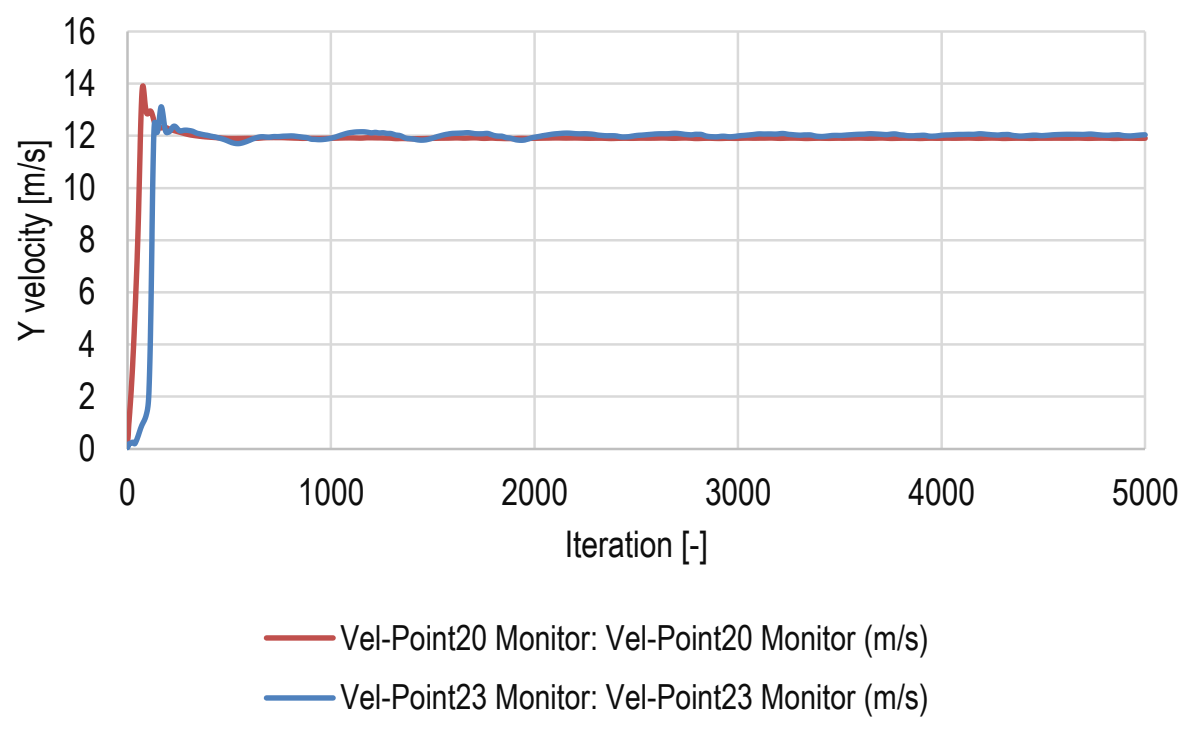

Figure 4-33: Velocity in Y direction at points 20 and 23 vs iteration number 
(a)

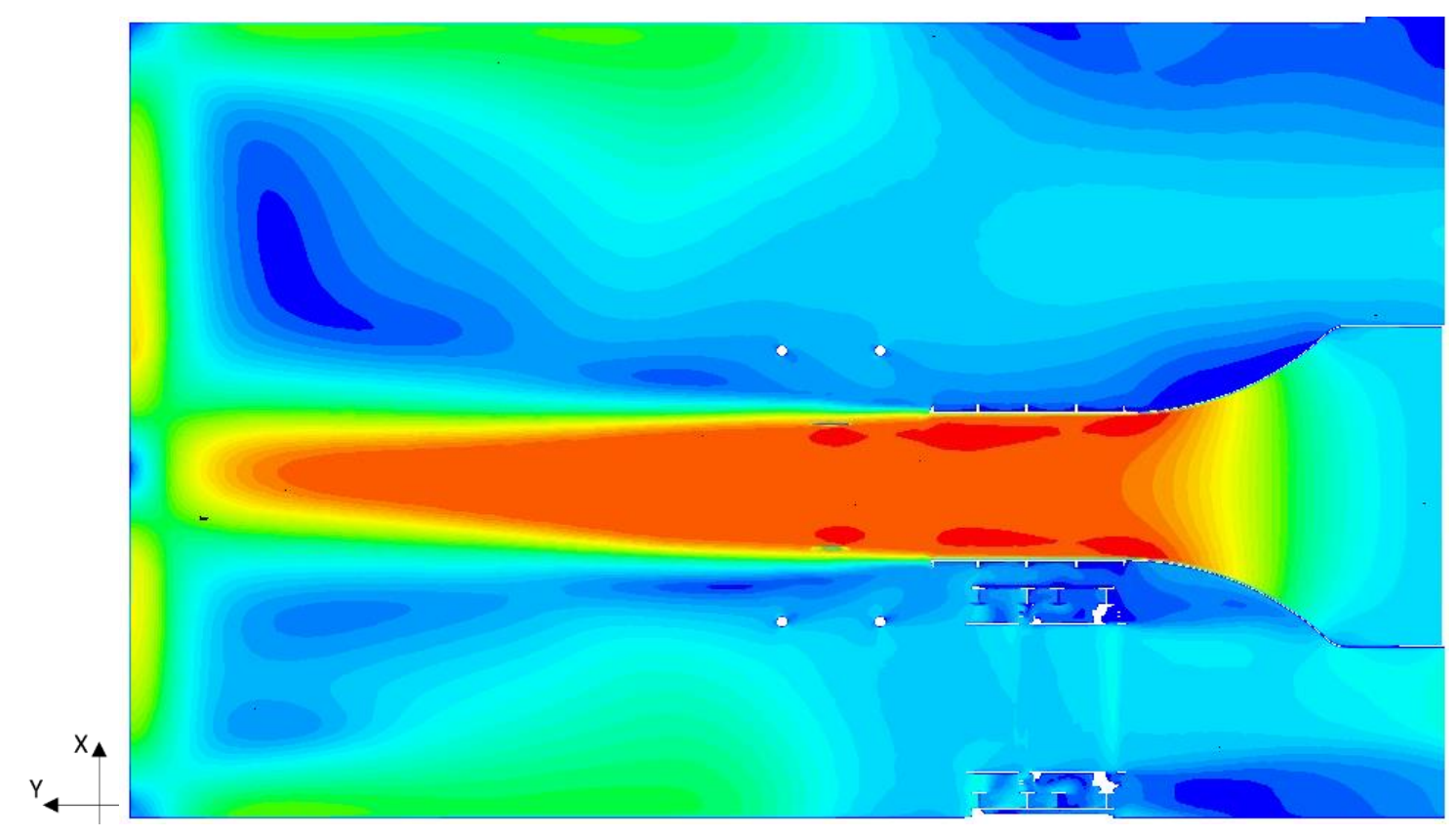

(b)

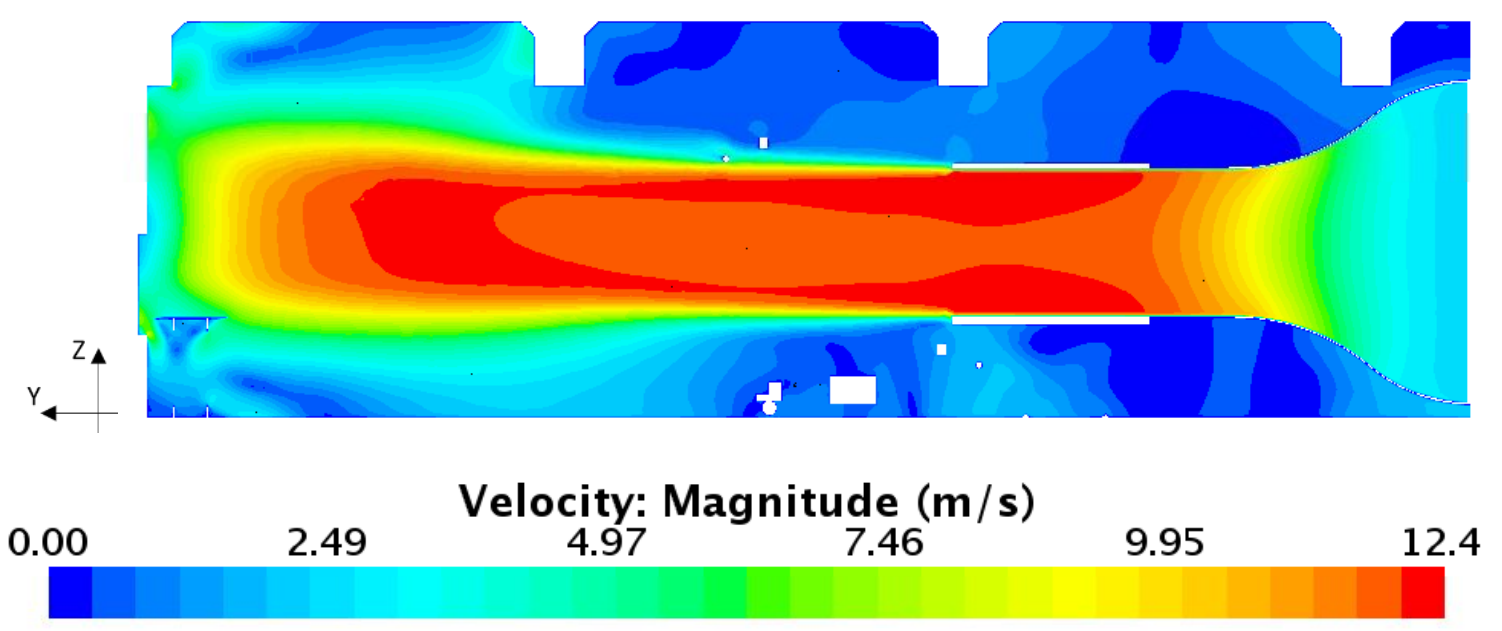

Figure 4-34: Velocity magnitude distribution on (a) horizontal, (b) vertical planes

\subsection{Comparison of computational and experimental results}

Results from several models described in detail in chapter 3 are compared. Table 4-6 combines average normal velocities on the wind tunnel screens in three models: with rotating reference frame and RANS solver with k- $\varepsilon$ turbulence model; with rotating reference frame and URANS solver with k- $\varepsilon$ turbulence model; and also a model with rotating fan and unsteady solver with LES turbulence model. The most significant quantity was the velocity on the last screen, number 9 (underlined in the table), because it was used as the inlet velocity to the simplified model. The comparison shows that the values are similar for all simulations, varying by less than $7 \%$. 
The models compared here are full room with recirculating flow leaving the fan, flowing down the wind tunnel, returning to the rear of the room and re-entering the fan through its inlets, as well as a model with simplified geometry, in which the recirculation is not present. The mass flow and mean velocity reached at the screens for a fixed fan speed results from a balance of energy imparted to the fluid by the fan, the flow energy losses caused by shear dissipation near walls, and the turbulence dissipation due to separation in flow around obstructions such as furniture. The variations arise primarily from the differences in the turbulence models and their ability to capture flow losses with reasonable accuracy.

Table 4-6: Average normal velocity on the wind tunnel screens

\begin{tabular}{|c|c|c|c|c|c|c|c|c|c|}
\hline $\begin{array}{l}\text { Screen } \\
\text { number }\end{array}$ & $\mathbf{1}$ & 2 & 3 & 4 & 5 & 6 & 7 & 8 & 9 \\
\hline $\begin{array}{c}\text { RANS k-e, } \\
\text { RRF }\end{array}$ & 10.719 & 10.718 & 8.254 & 6.247 & 4.820 & 3.665 & 2.714 & 2.484 & $\underline{2.472}$ \\
\hline $\begin{array}{l}\text { URANS, } \\
\text { k- } \varepsilon, \\
\text { rotating } \\
\text { fan }\end{array}$ & 11.092 & 11.092 & 8.545 & 6.466 & 4.988 & 3.793 & 2.809 & 2.571 & 2.559 \\
\hline LES, RRF & 10.380 & 10.366 & 7.973 & 6.031 & 4.655 & 3.540 & 2.623 & 2.404 & 2.388 \\
\hline
\end{tabular}

Figure 4-35 presents a comparison of velocity magnitude along lines (a) I, (b) II, (c) III and Figure 4-36 compares velocity magnitude along lines (a) IV, (b) V, (c) VI, and (d) VII. Figure 2-2 shows the positions of the probe lines in the domain. The measurements were taken at a height of $7 \mathrm{ft}$. In addition to the model results, the starred points show means of measured values in the laboratory with the wind tunnel in operation. It is worth restating here that unsteady oscillations of the readings were observed with significant amplitudes at the locations closer to the wall (see Figure 4-6). The average values and standard deviations of velocity at points 20 and 23 are combined in Table 2-1 and Table 2-2. 
(a)
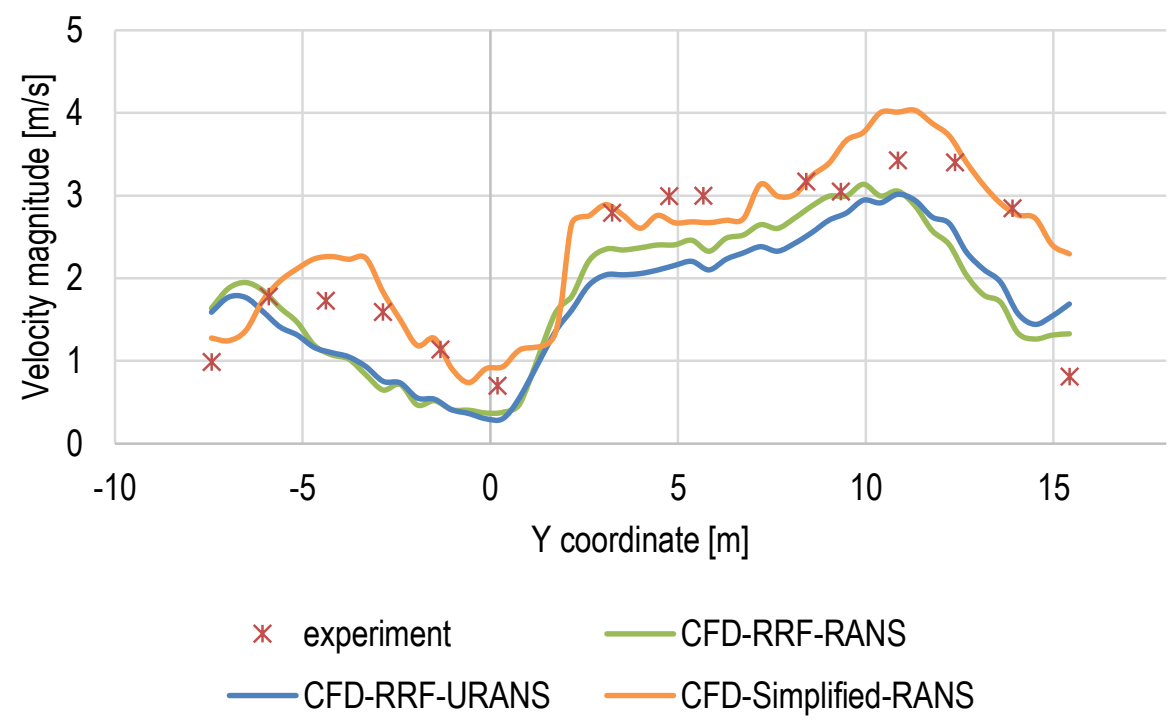

(b)
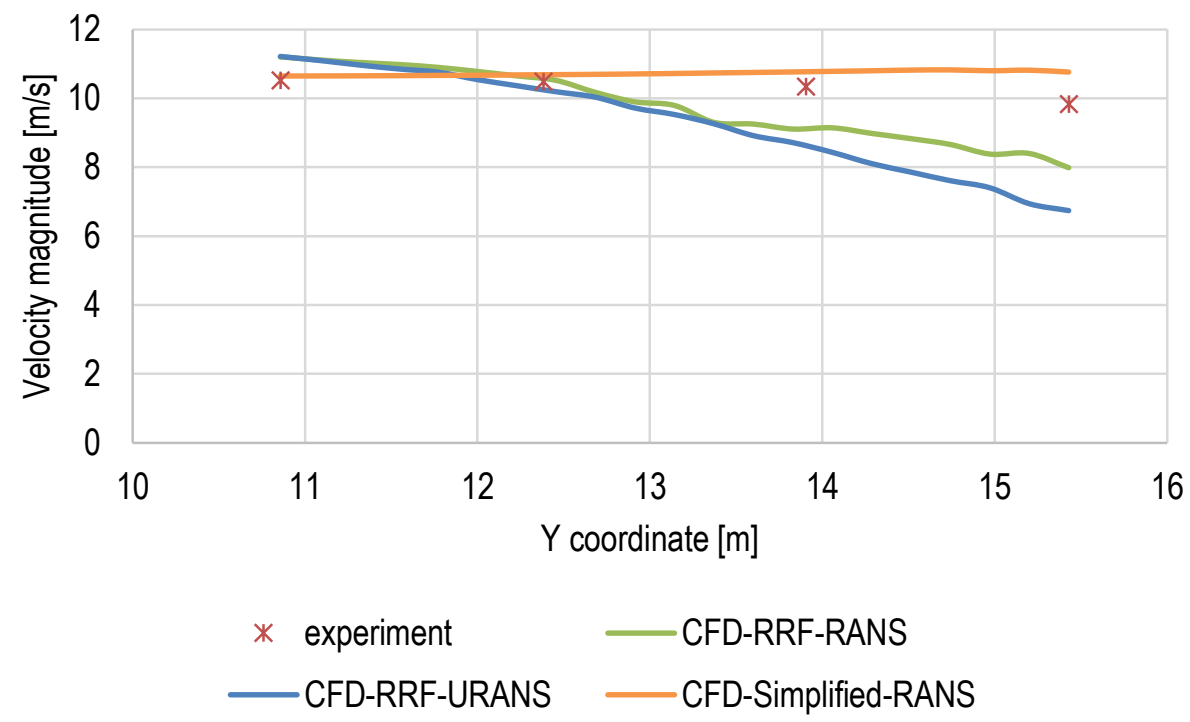
(c)
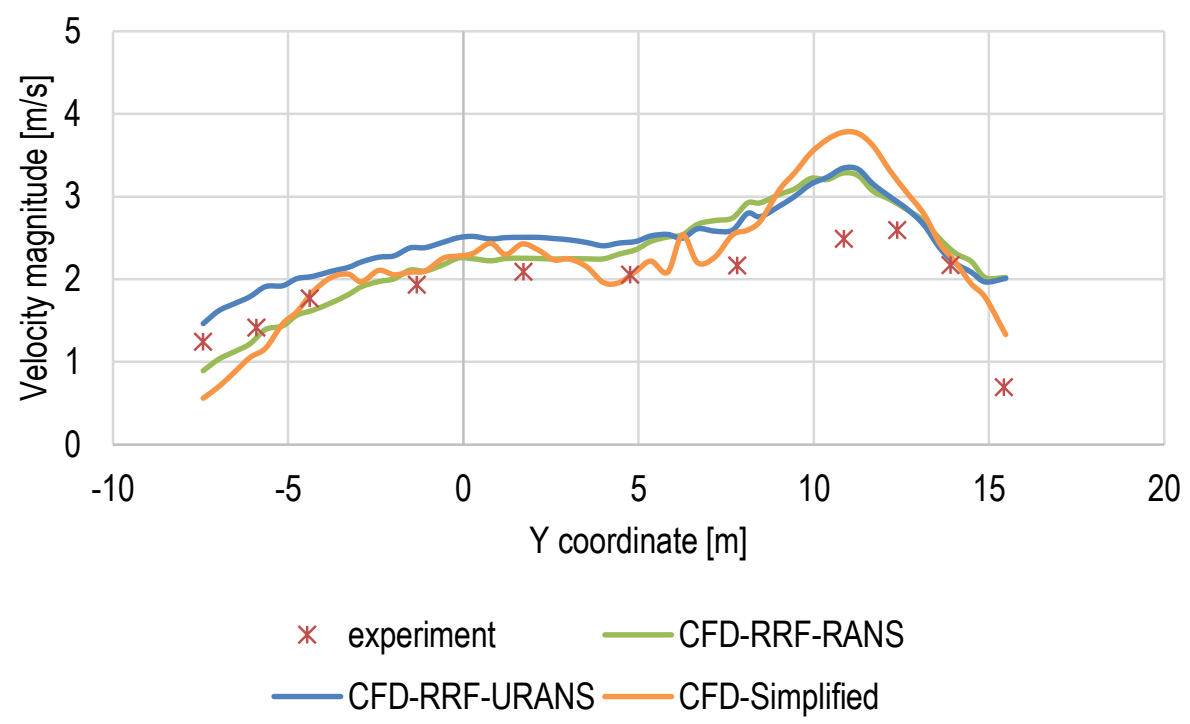

Figure 4-35: Comparison of velocity magnitude along lines a) I, b) II, c) III

(a)

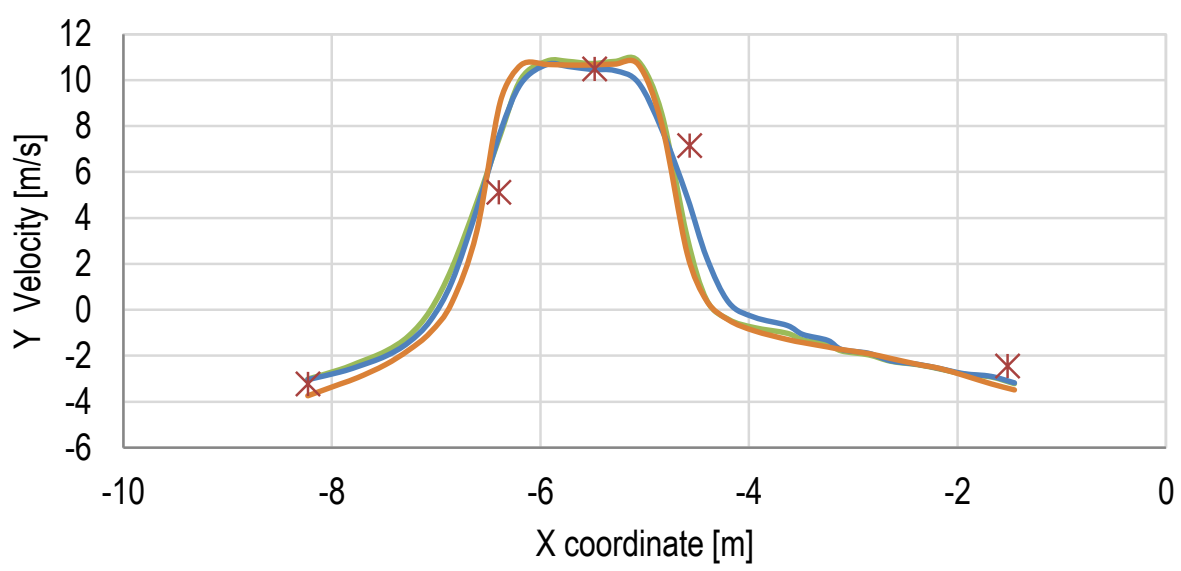

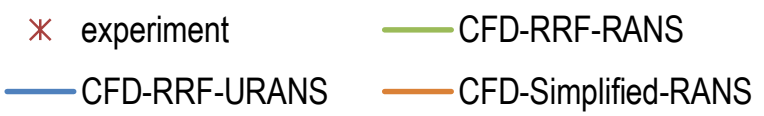


(b)
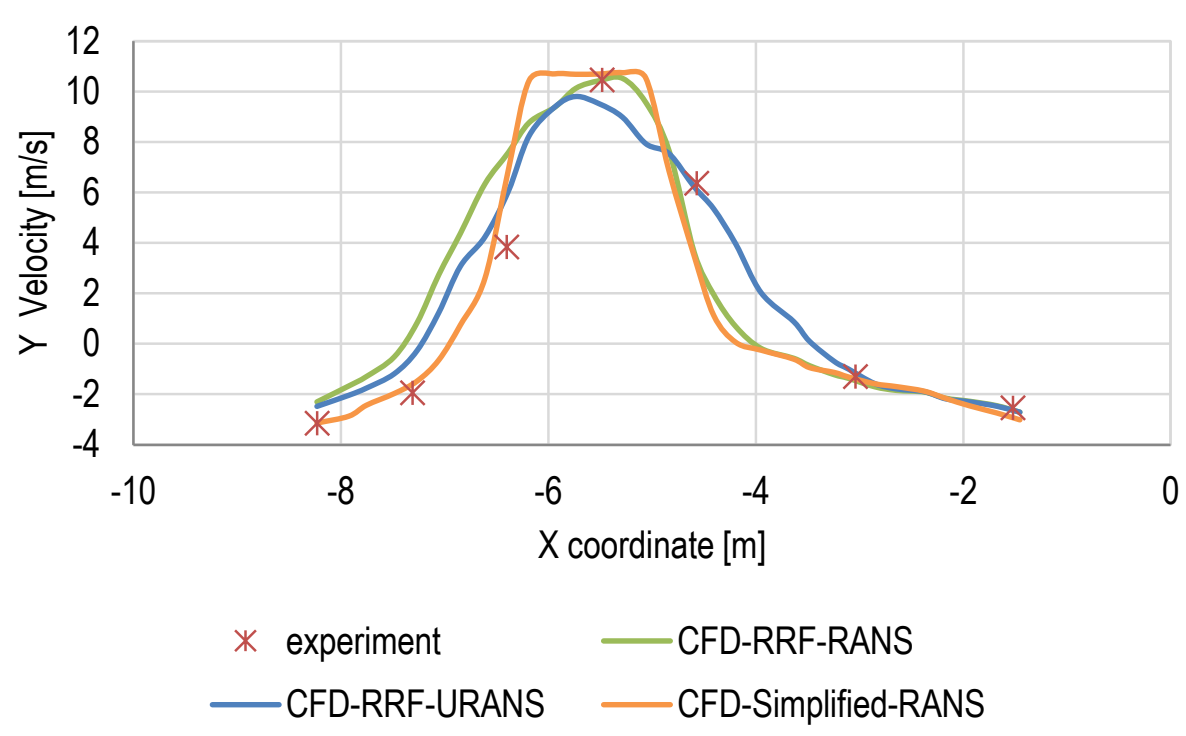

(c)

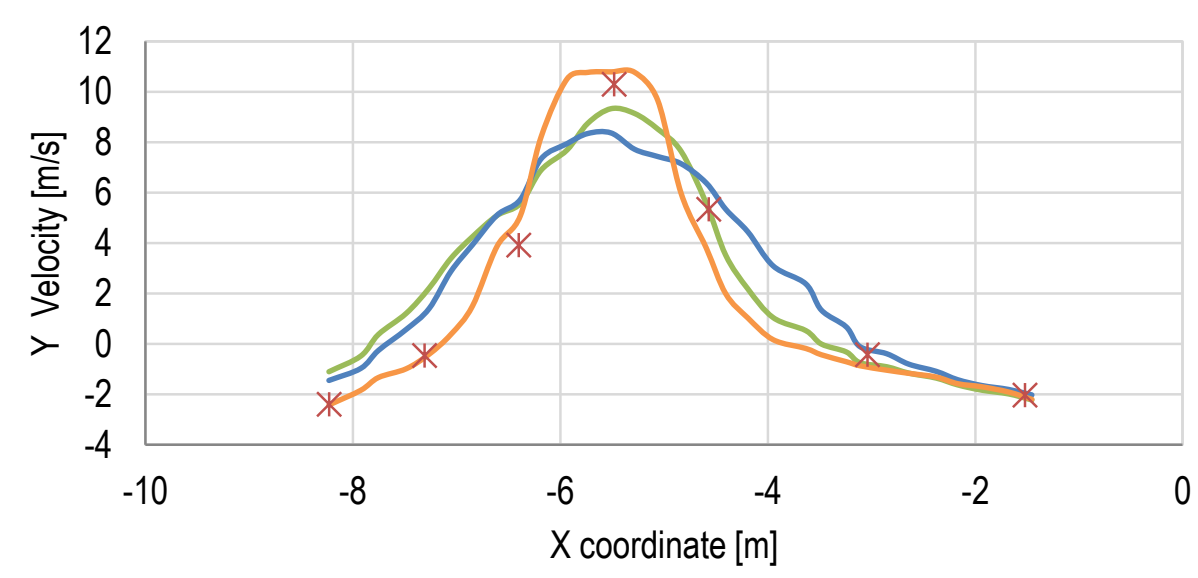

* experiment C CFD-RRF-RANS

—CFD-RRF-URANS CCFD-Simplified-RANS 
(d)

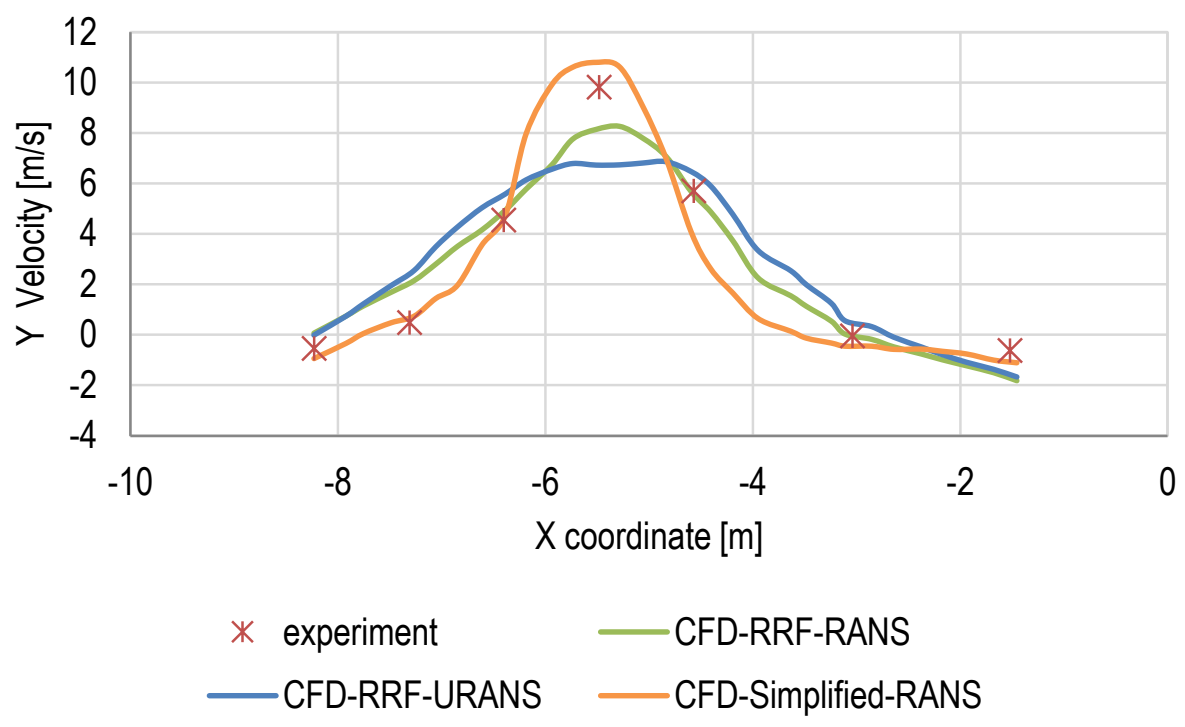

Figure 4-36: Y velocity along lines a) IV, b) V, c) VI, d) VII

Several models were validated additionally by comparing the time averaged measured values of the flow velocity in the flow direction with the computed results. The location and name convention of the measuring points is shown in Figure 2-3. The measurements were taken at three probe points at the wind tunnel extension, inside and right in front of it, and on a vertical plane in front of the extension. During the measurements the fan was run at various speeds. In this report, three representative speed values were taken for consideration, 117, 223, and $292 \mathrm{rpm}$. The computational results obtained with the rotating reference model were averaged over 1000 iterations. The velocities at considered probes in the model with simplified geometry converged to constant values and therefore the averaging was not necessary.

Figure 4-37 shows a comparison of experimental and computational velocities in the flow direction at probes 1, 2, and 3, at fan speed (a) $117 \mathrm{rpm}$, (b) $223 \mathrm{rpm}$, and (c) $292 \mathrm{rpm}$ for the model with rotating reference frame and RANS solver. Figure 4-38 shows a comparison of experimental and computational velocity magnitudes at probe plane 4 , at the same fan speeds for the model with rotating reference frame and RANS solver. Figure 4-39 illustrates the velocity components. Figure 4-40, Figure 4-41 and Figure 4-42 present the same set of results for the model with simplified geometry.

It's worth noting that the measurements give a range of time averaged values for probes 1,2, and 3, as seen in Figure 4-37 and Figure 4-40. The measurements were made while a pitot tube was moved to a series of locations on probe plane 4, see Figure 2-3, to assess the sensitivity of measurements. The presence of pitot tube measurement probes was not modeled in the CFD analysis. Figures show that both CFD models give velocities that are close to the measured values at all probes and at all selected fan speeds. Figure 4-38, Figure 4-39, Figure 4-41, and Figure 4-42 illustrate velocity magnitude and components obtained in CFD simulations and the velocity measured in the wind tunnel. The velocity magnitude and velocity in the flow direction are very 
close which suggests that the flow is almost parallel. It maintains an almost constant high value in the central part of the wind tunnel exit and drops only in the vicinity of the edges of the structure, which agrees well with the experiment.

(a)

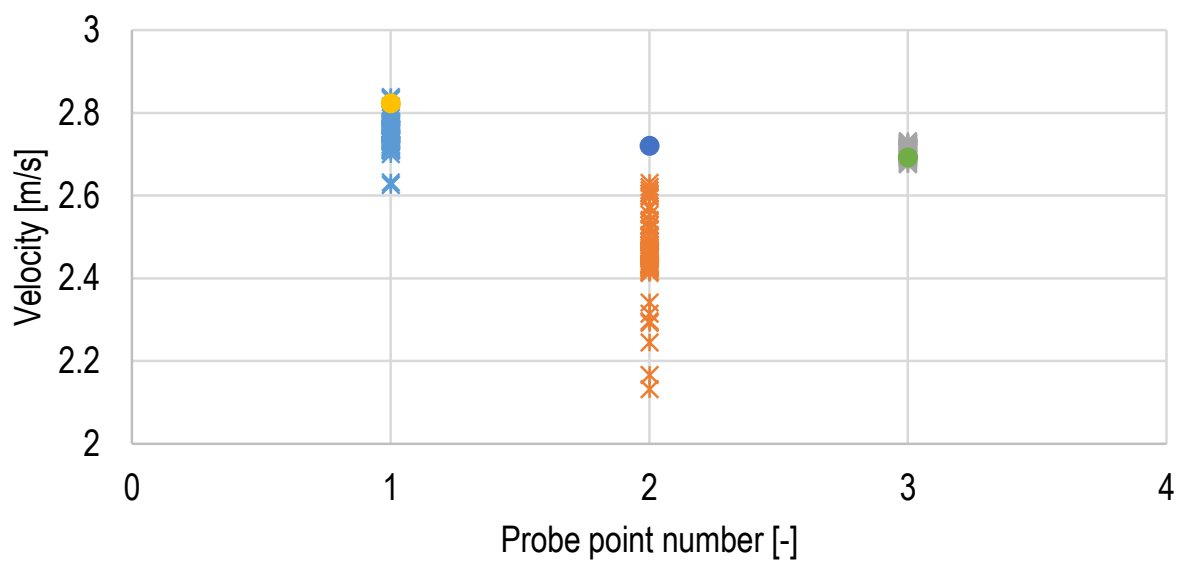

$$
* \text { Probe } 1 \text { - experiment } \odot \text { Probe } 1 \text { - CFD } \quad * \text { Probe } 2 \text { - experiment }
$$

- Probe 2 - CFD $\quad *$ Probe 3 - experiment - Probe 3 - CFD

(b)

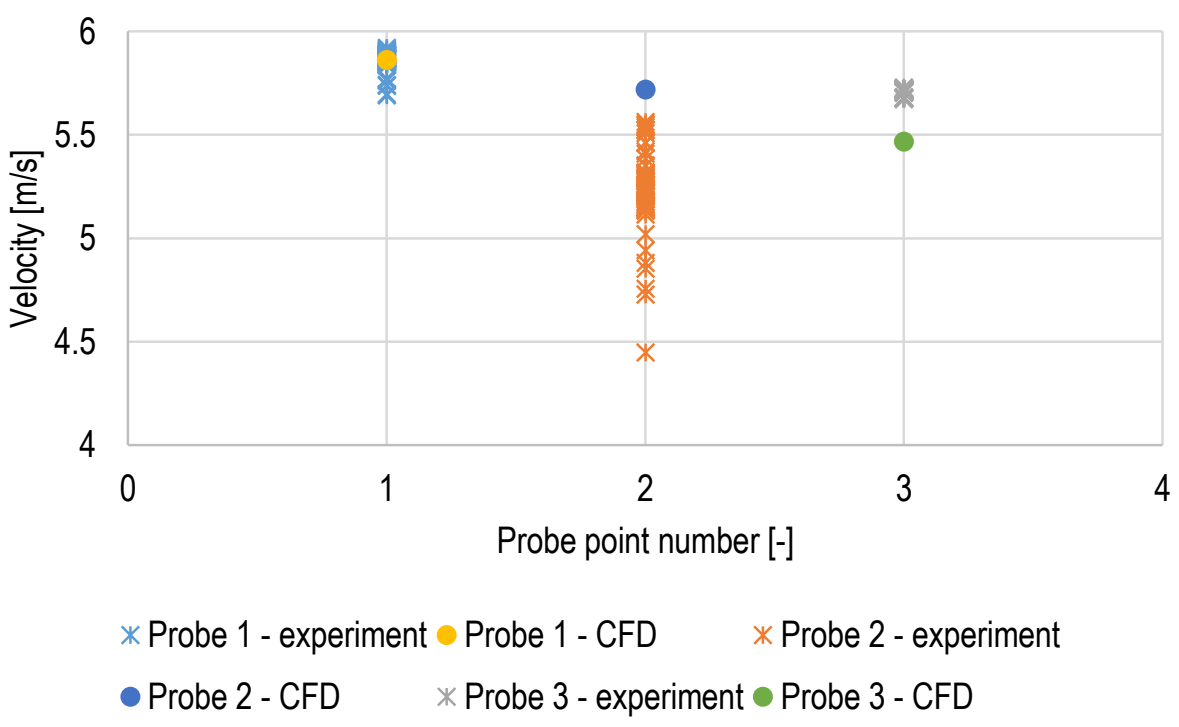


(c)

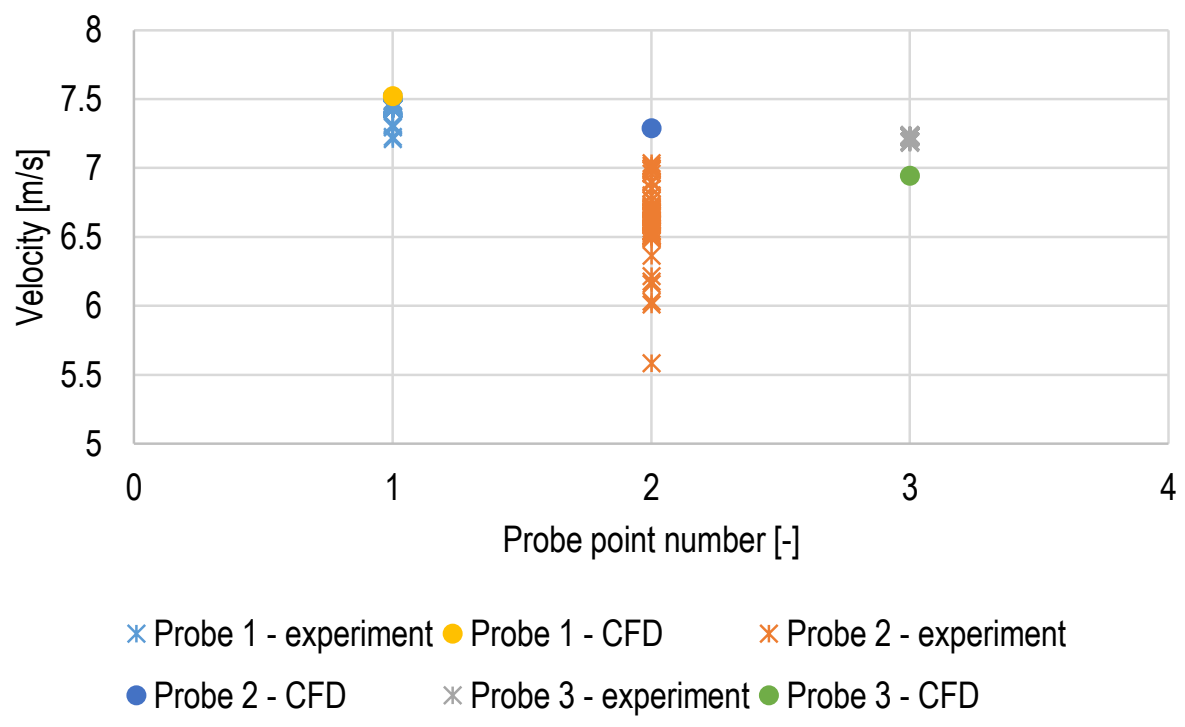

Figure 4-37. Comparison of experimental and computational velocities in the flow direction at probes 1, 2, and 3, at fan speed (a) $117 \mathrm{rpm}$, (b) $223 \mathrm{rpm}$, (c) $292 \mathrm{rpm}$. Model with rotating reference frame, RANS solver.

(a)

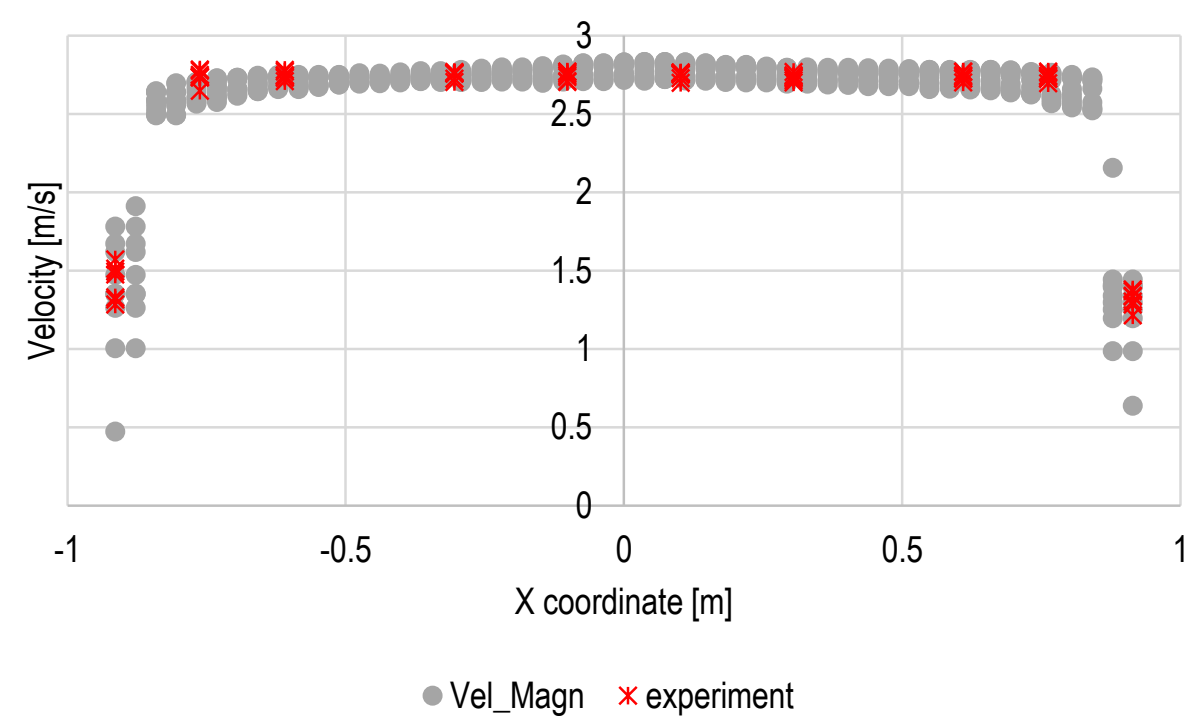


(b)

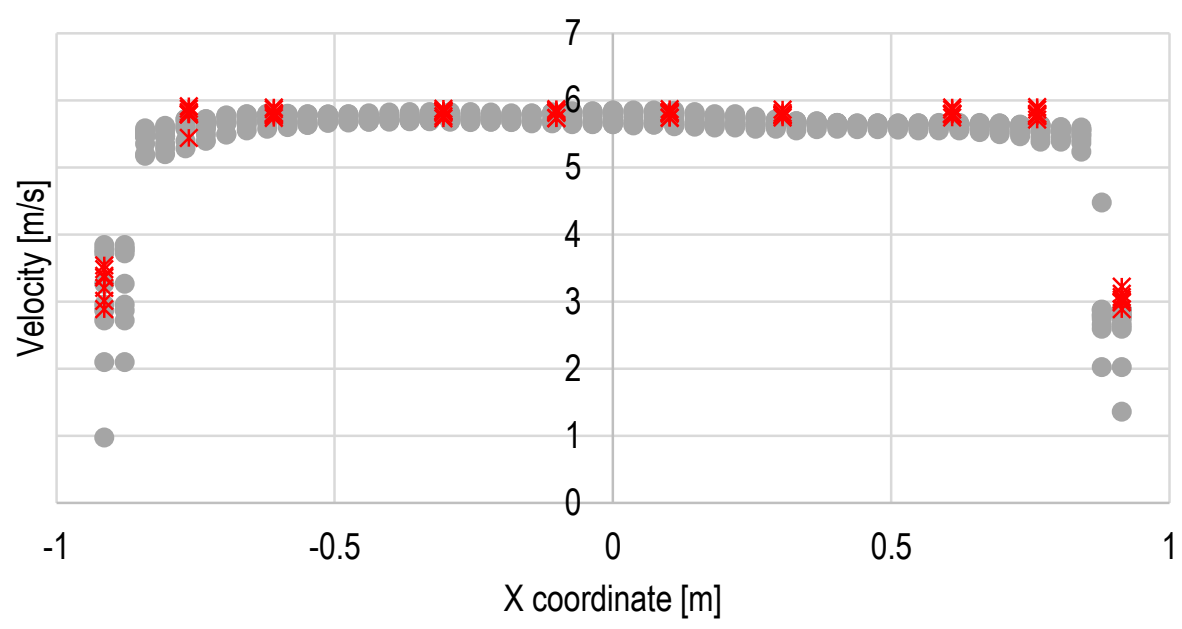

- Vel_Magn * experiment

(c)

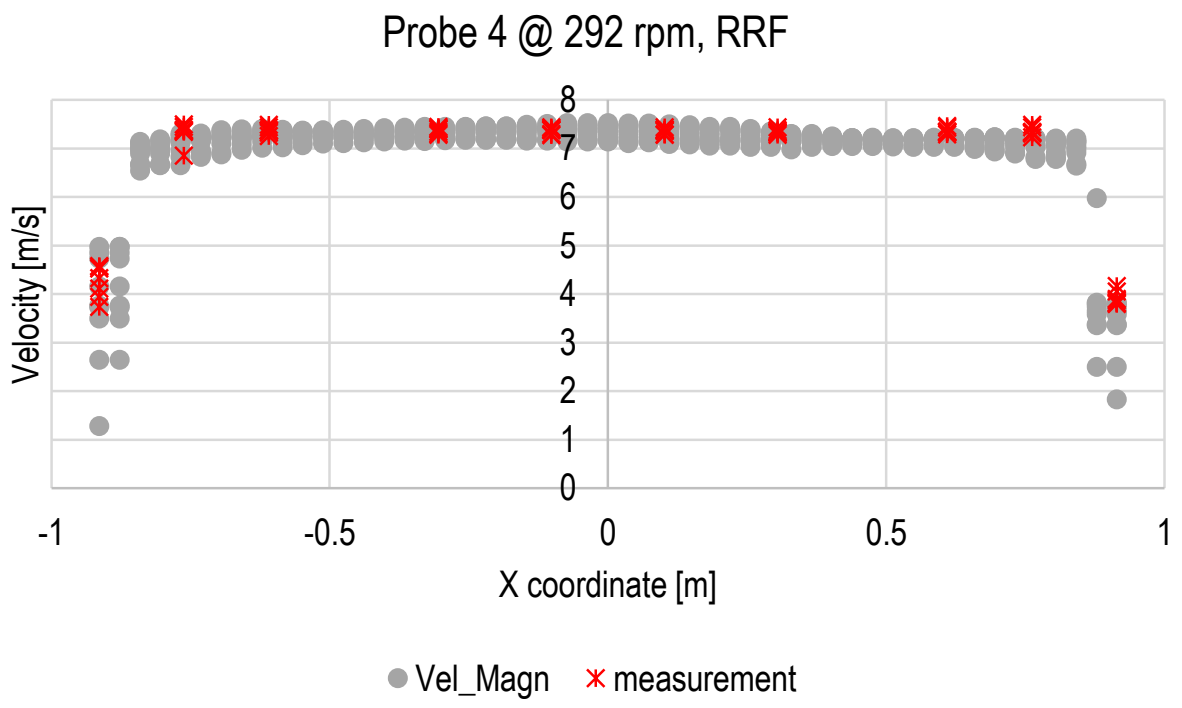

Figure 4-38. Comparison of experimental and computational velocity magnitudes at probe plane 4 (a) 117 rpm, (b) $223 \mathrm{rpm}$, (c) $292 \mathrm{rpm}$. Model with rotating reference frame, RANS solver 
(a)

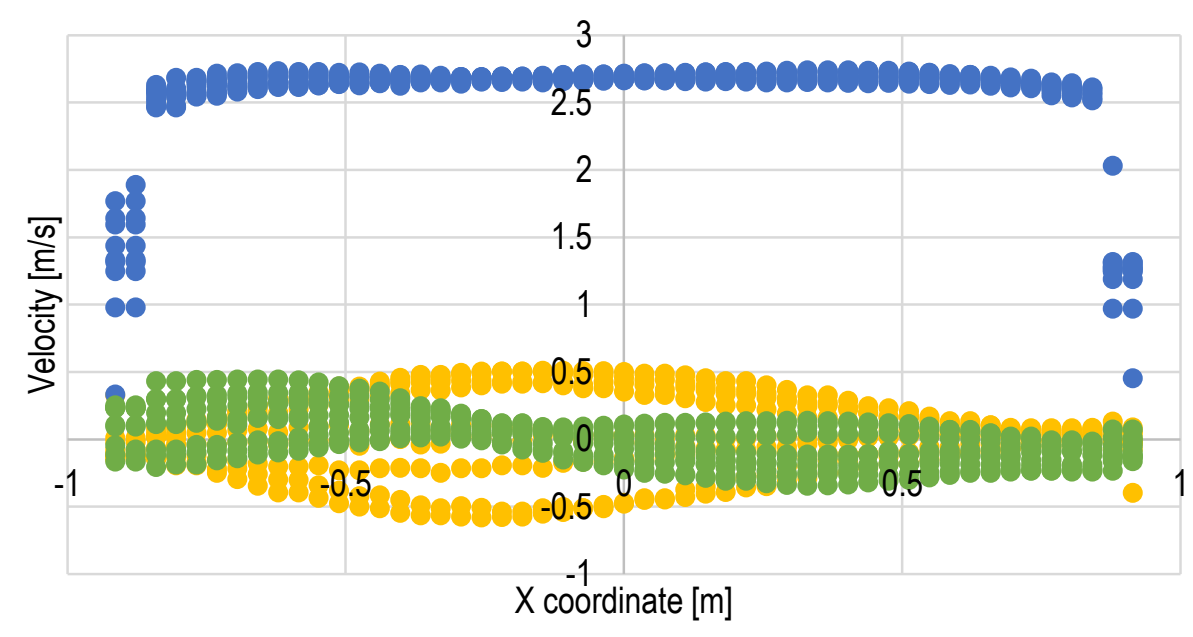

- Vel_X • Vel_Y • Vel_Z

(b)

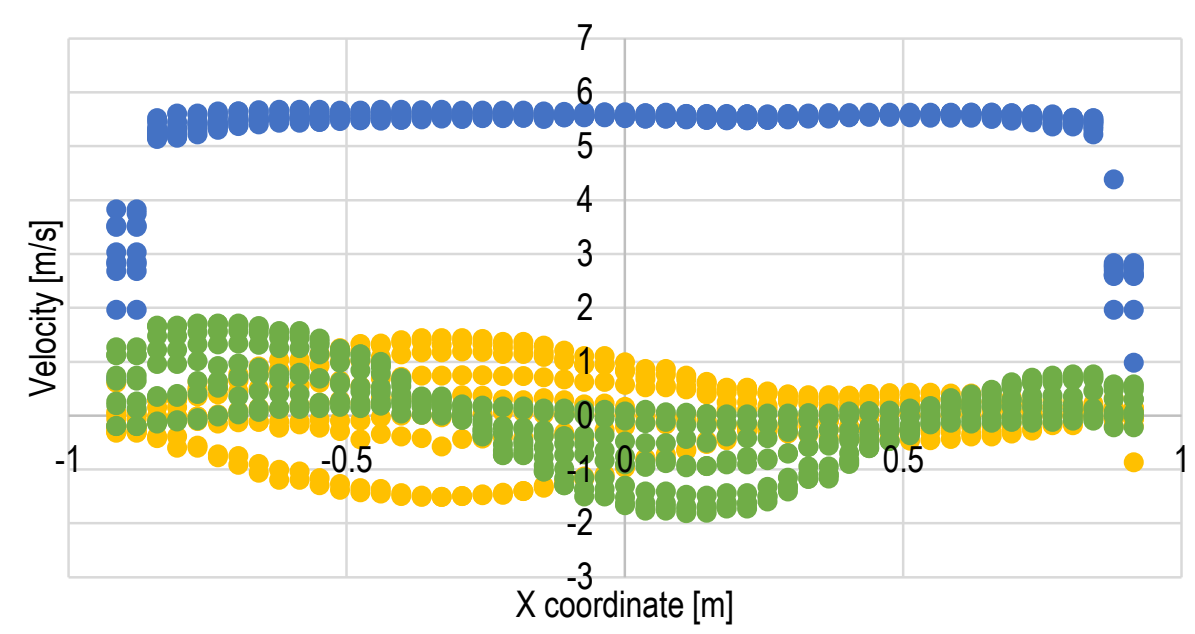

- Vel_X $\bullet V e I_{-} Y \bullet V e l \_Z$ 
(c)

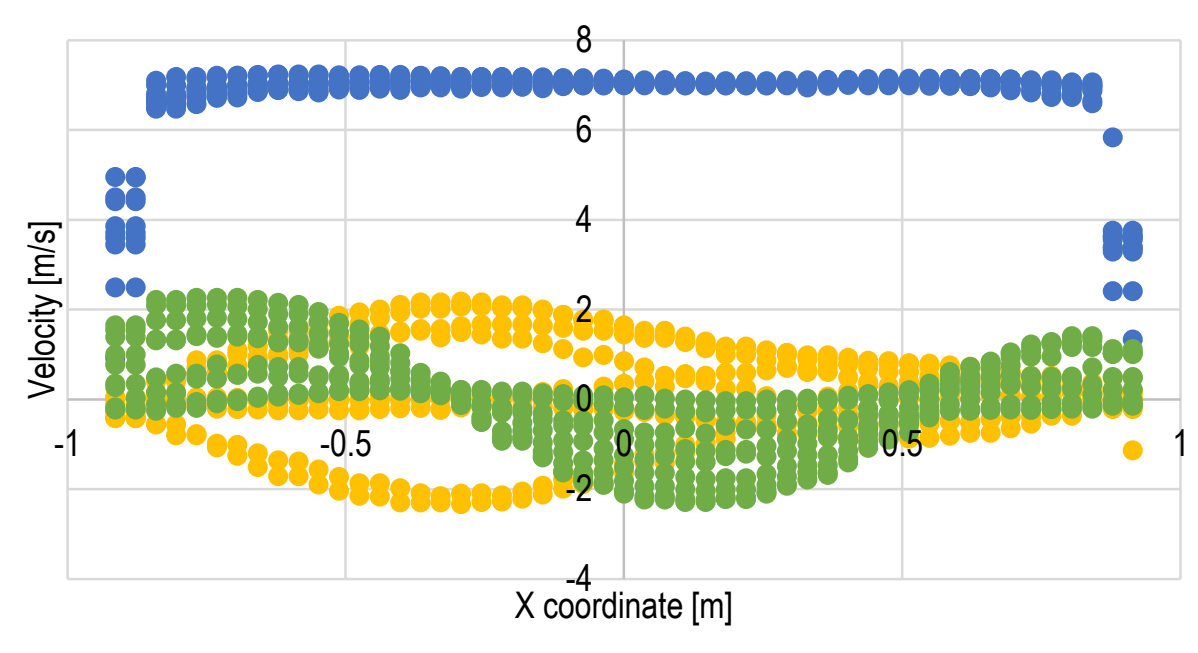

- Vel_X $\bullet$ Vel_Y $\bullet$ Vel_Z

Figure 4-39. Computational velocity components at probe plane 4 (a) $117 \mathrm{rpm}$, (b) $223 \mathrm{rpm}$, (c) $292 \mathrm{rpm}$. Model with rotating reference frame, RANS solver

(a)

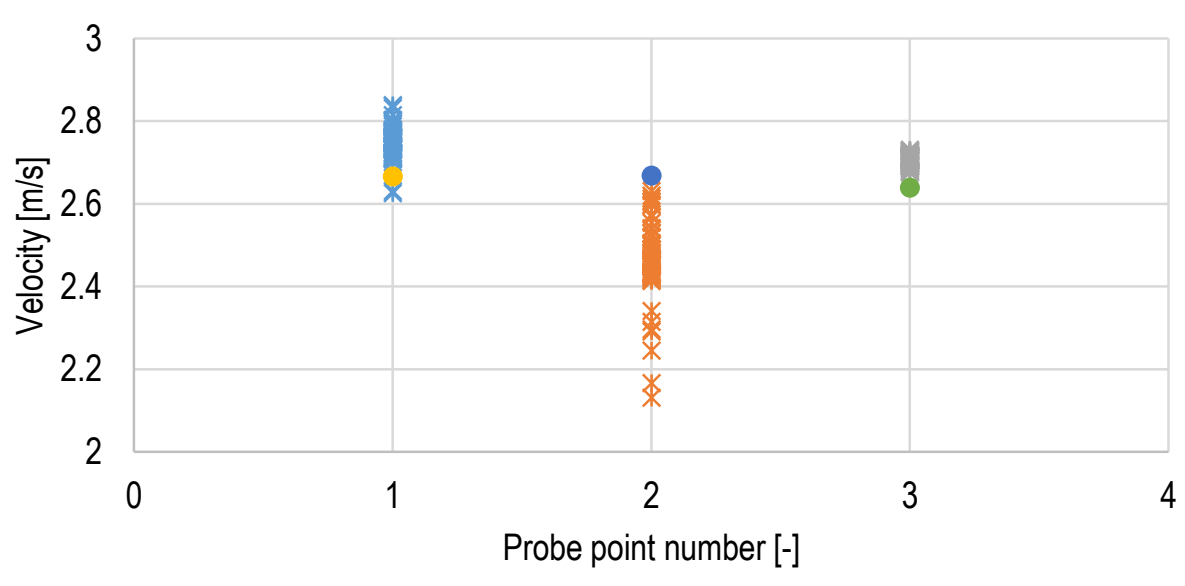

* Probe 1 - experiment $\circ$ Probe 1 - CFD $\quad *$ Probe 2 - experiment

- Probe 2 - CFD $\quad *$ Probe 3 - experiment $\bullet$ Probe 3 - CFD 
(b)

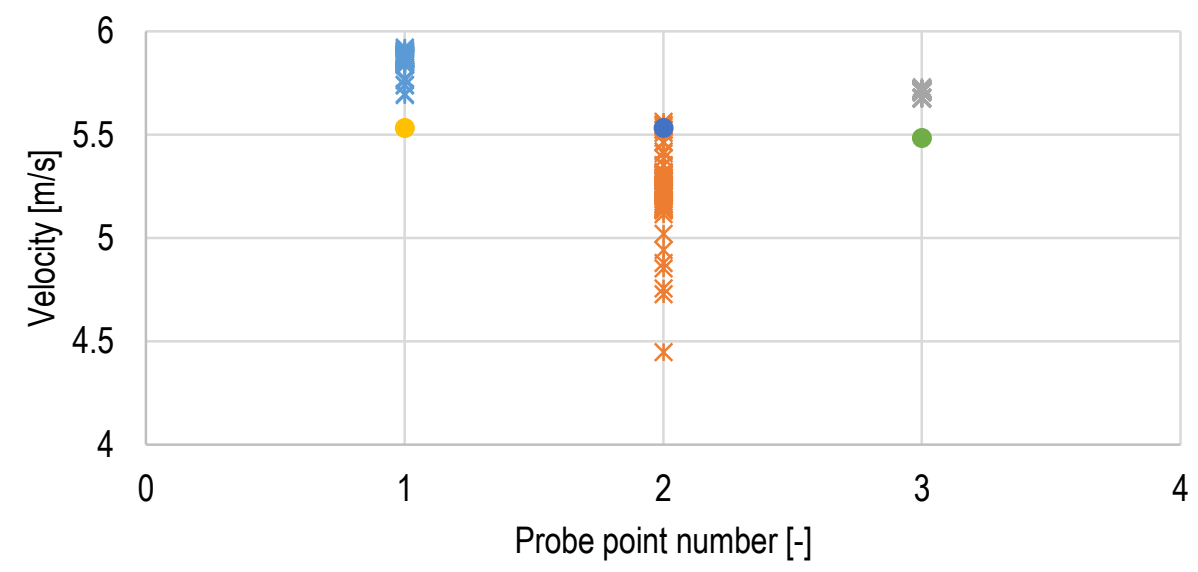

* Probe 1 - experiment $\odot$ Probe 1 - CFD $\quad *$ Probe 2 - experiment

- Probe 2 - CFD $\quad *$ Probe 3 - experiment $\bullet$ Probe 3 - CFD

(c)

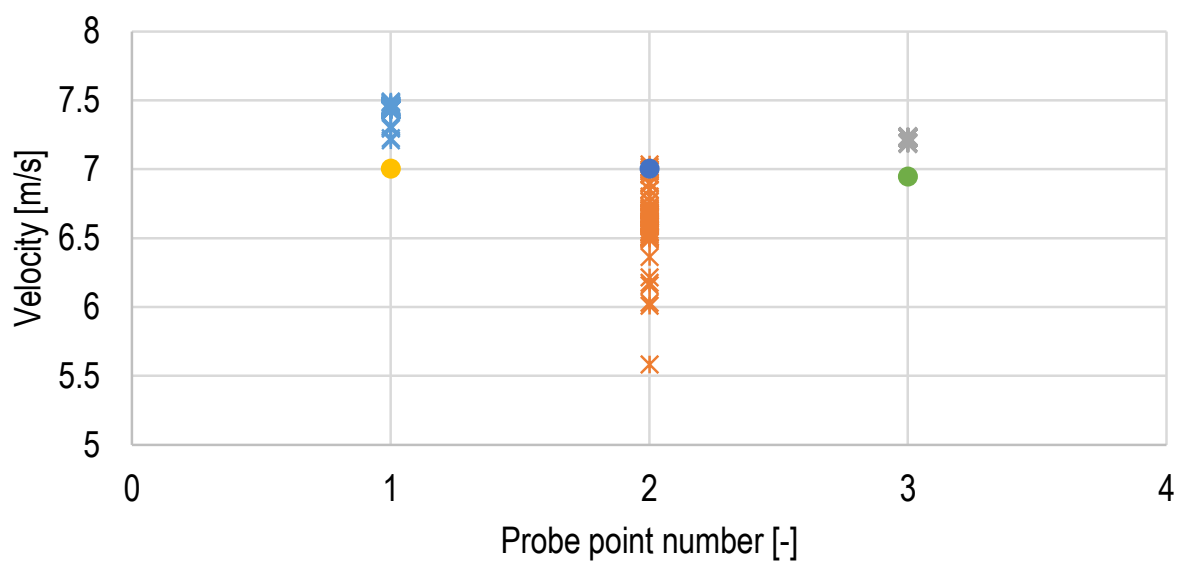

* Probe 1 - experiment $\odot$ Probe 1 - CFD $\quad *$ Probe 2 - experiment

- Probe 2 - CFD $\quad$ P Probe 3 - experiment $\bullet$ Probe 3 - CFD

Figure 4-40. Comparison of experimental and computational velocities in the flow direction at probes 1, 2, and 3, at fan speed (a) $117 \mathrm{rpm}$, (b) $223 \mathrm{rpm}$, (c) $292 \mathrm{rpm}$. Model with simplified geometry, RANS solver. 
(a)

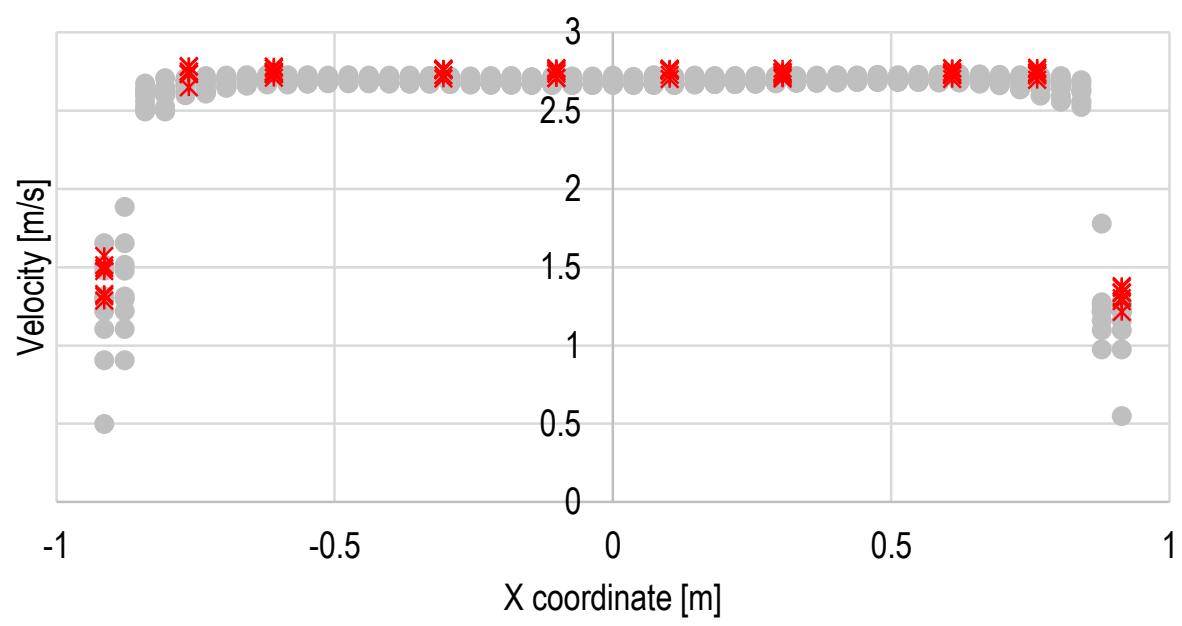

Vel_Magn *experiment

(b)

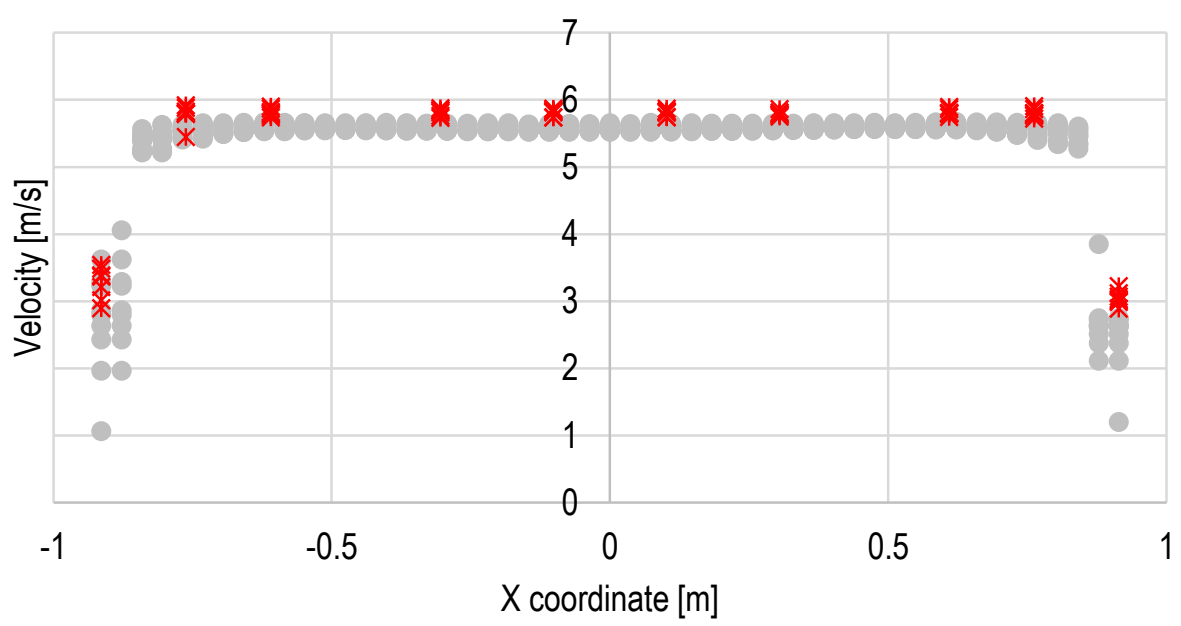

Vel_Magn $*$ measurement 
(c)

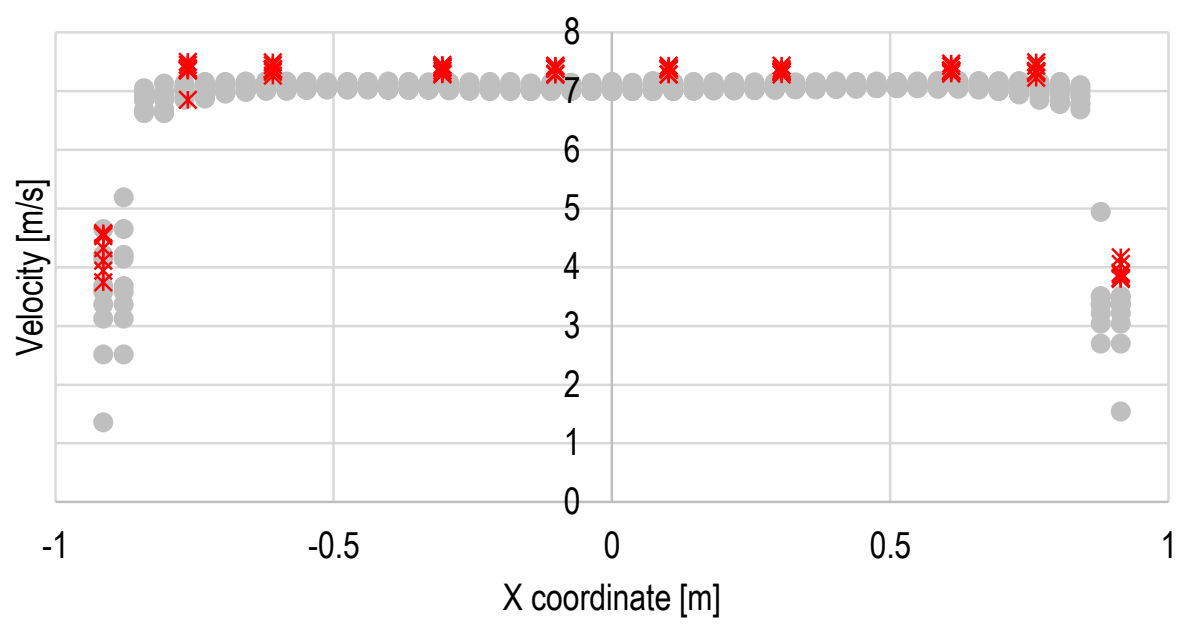

Vel_Magn $\quad *$ measurement

Figure 4-41. Comparison of experimental and computational velocity magnitudes in the flow direction at probe plane 4, at fan speed (a) $117 \mathrm{rpm}$, (b) $223 \mathrm{rpm}$, (c) $292 \mathrm{rpm}$. Model with simplified geometry, RANS solver.

(a)

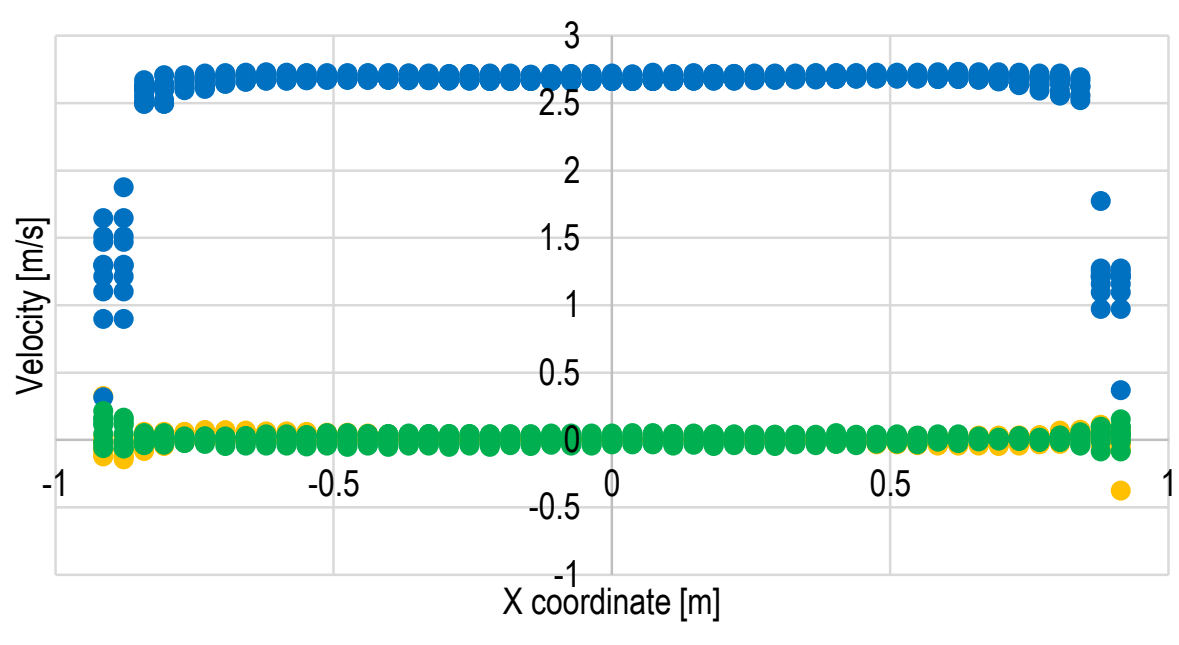

-Vel_X •Vel_Y •Vel_Z 
(b)

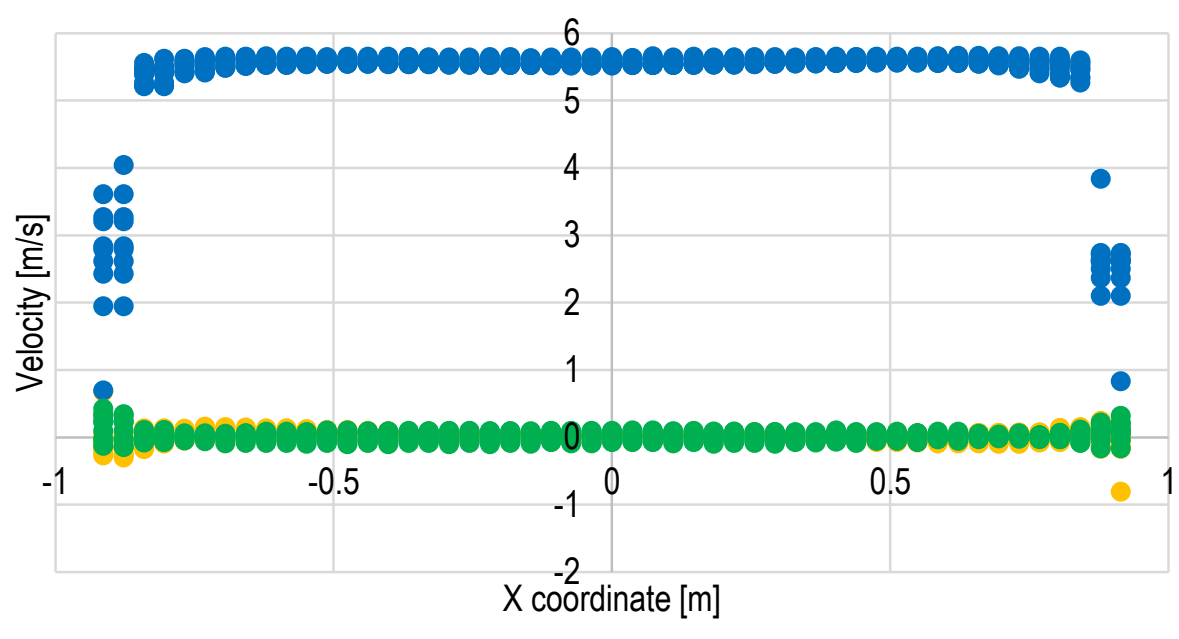

-Vel_X $\bullet$ Vel_Y $\bullet V e l \_Z$

(c)

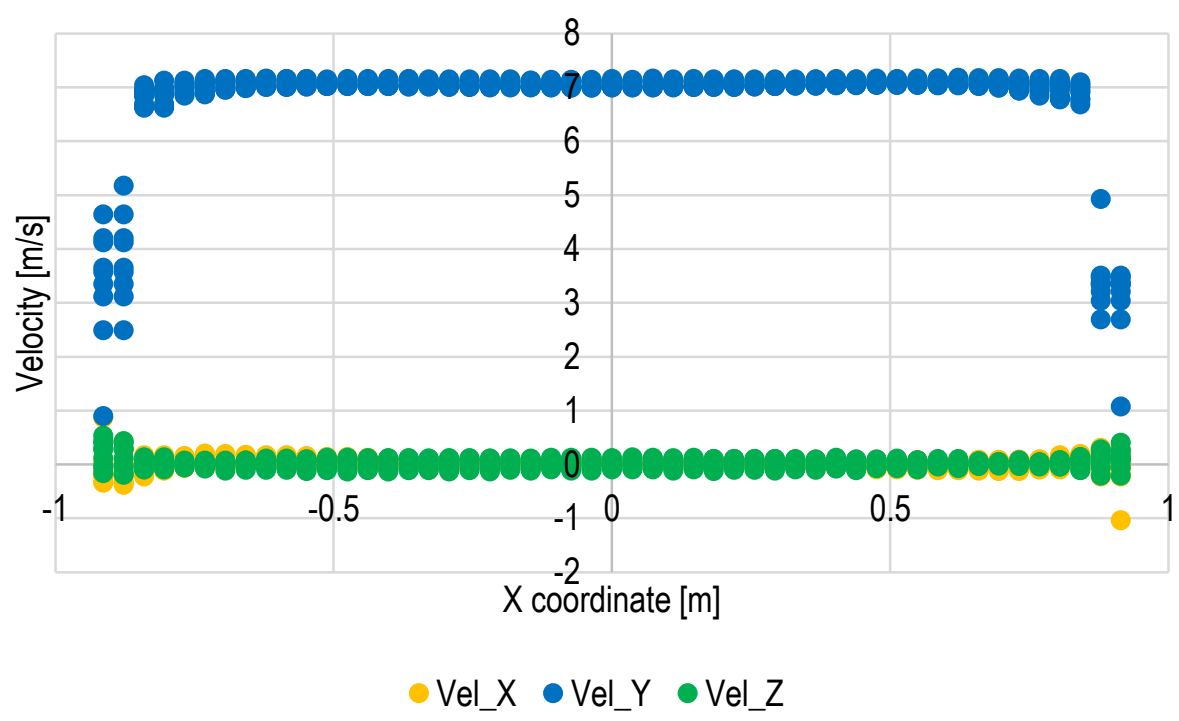

Figure 4-42. Computational velocity components in the flow direction at probe plane 4, at fan speed (a) 117 rpm, (b) 223 rpm, (c) $292 \mathrm{rpm}$. Model with simplified geometry, RANS solver.

\section{Summary}

The goal of this work is to create a "virtual laboratory" in CFD environment that could be used to run larger parametric tests or extend the experimental testing beyond the conditions available in the lab. This report describes the development of full scale CFD models of the Turner-Fairbank Aerodynamics Laboratory with the wind tunnel, equipment, furniture and other objects inside. Models with different levels of complexity were tested and the results were compared to the experimental data. 
The proposed models included: a full model including both the interior and exterior of the wind tunnel with a rotating fan, a full model with a rotating reference frame fan model, and simplified cases without the fan and interior of the wind tunnel, but with appropriate inflow and outflow boundary conditions.

The results were compared against experimental velocity measurements at various locations around the room inside and outside the testing area. CFD results for full models match well with the experimental data in the jet region. The air flow is uniform there, with no influence from the surrounding equipment and furniture. Discrepancies in the flow character appear in areas of less importance, in the proximity of walls and on the sides of the room. In the simulations with closed air flow, i.e. in the models with a rotating fan or rotating reference frame fan model, the jet slowed down and slightly changed direction. Also, oscillations of the flow were noticeable, even in RANS computations. The run times ranged from a few days up to two weeks on 4 (up to 6) 16-core compute nodes.

In order to reduce the computational time and save resources, simplifications of the models were proposed. The inside of the wind tunnel was removed from the domain, the last (furthermost from the fan) screen was changed into a velocity inlet boundary and the surfaces where the air enters the fan were modified into a pressure outlet boundary. Breaking the loop eliminated the oscillations of the flow as well as the decrease in velocity close to the walls. It also reduced the computational time to a few hours on the same number of cores as before.

\section{Recommendations for application of the models}

As described in previous sections, this project developed a set of models of the Turner-Fairbank Highway Research Center Aerodynamics Laboratory large wind tunnel including the full geometry of the room containing the wind tunnel. These recommendations cover the models that appear to be the best choice in terms of both cost of use and accuracy, for various applications to testing the performance of structures in wind conditions that the models may be used to analyze. Two classes of application testing may be identified: (1) The models may be used to fill in gaps in actual physical tests run with the wind tunnel in the laboratory or extend tests beyond fan speeds that are possible. In this case, having conditions in the test section that match actual physical conditions in the test section zone of the wind tunnel laboratory is likely to produce the results that match quantitative results and the trends of the physical wind tunnel tests most closely. Whether or not the model matches air velocity measurements made in the laboratory room that are considerably far away from the test section is less important. (2) The models may be used either with the aerodynamics laboratory geometry, or with a simpler geometry that is an empty box with pressure outlet side boundary conditions representing an ideal wind tunnel, or finally in a more complex geometry that would come close to some specific field conditions at full scale. For these second uses, it is confidence and knowledge of the capability of the CFD software to produce accurate results using appropriate computational grids, turbulence models, and solver settings, and the confidence interval for those results that is of importance. 


\subsection{Recommendations for turbulence models}

Roughly in order of analysis cost in use of high performance cluster resource usage, lowest to highest, are Reynolds Averaged Navier-Stokes (RANS) equations turbulence models, unsteady RANS (URANS), k-omega SST, and Large Eddy Simulation (LES) (for the full formulation, see [2]). LES analysis captures the formation and evolution of the large eddy structures that contain most of the turbulent energy down to the eddy size that can be resolved in the computational grid. The diffusion terms in the partial differential equations in LES models use the actual material viscosity. This provides a basis for the most accurate transient physical response and evolution of flow structures to obstructions in the flow field.

In RANS and URANS models, the averaging process used to obtain the averaged equations leaves cross correlations of fluctuating velocities that are variations caused by eddies that can quickly transport momentum, heat, or chemical species across streamlines from one side of the flow field to the other. These cross correlations, known as Reynolds stresses are new unknowns and would need a set of new independent equations for them in order to solve for their values. The two equation turbulence models model the Reynolds stress terms with an eddy viscosity, $\mu_{t}$ :

$$
-\rho\left\langle u_{i}^{\prime} u_{j}^{\prime}\right\rangle=\mu_{t}\left(\frac{\partial U_{i}}{\partial x_{j}}+\frac{\partial U_{j}}{\partial x_{i}}\right) .
$$

The angle brackets denote averaging, the primes are turbulent fluctuating quantities, and $\mu_{t}$ is the turbulent eddy viscosity. In the k-epsilon turbulence model:

$$
\mu_{t}=C_{\mu} \rho \frac{k^{2}}{\epsilon} .
$$

The RANS equations are closed by substituting the eddy viscosity diffusion terms for the Reynolds stress terms, which when combined with the diffusion terms based on material viscosity yield diffusion terms of the form:

$$
\left(\mu_{t}+\mu\right)\left(\frac{\partial U_{i}}{\partial x_{j}}+\frac{\partial U_{j}}{\partial x_{i}}\right)=\mu_{e f f}\left(\frac{\partial U_{i}}{\partial x_{j}}+\frac{\partial U_{j}}{\partial x_{i}}\right)
$$

Note that the turbulent viscosity is directly proportional to the turbulent kinetic energy, $k$, squared and inversely proportional to the turbulence dissipation rate, $\varepsilon$. In regions of high turbulent kinetic energy, the turbulent viscosity can be quite large, three orders of magnitude, or more, greater than the material viscosity of air. Under these conditions, the turbulent viscosity dominates the diffusion transport which allows variations in the flow to be transported across the mean streamlines in the primary flow direction to the sides of the domain very quickly. Thus in this type of turbulence model, a high diffusion rate mimics the effects eddy rotational action. The high turbulent viscosity in the model also has the effect of laminarizing the mean flow. To see why, consider a Reynolds number based on the turbulent viscosity, $\mu_{t}$ :

$$
R e_{t}=\frac{\rho U h}{\mu_{t}}
$$


With the turbulent viscosity in the model three orders of magnitude, or more, greater than the air viscosity, the effective turbulent Reynolds number of the mean flow, $R \boldsymbol{e}_{t}$, is about three orders of magnitude smaller. This means that if the Reynolds number for flow past an obstruction is 30,000 then the effective Reynolds number for the mean flow solved with the RANS model would be about 300, or laminar, for many bluff bodies.

The RANS and URANS turbulence models use the least amount of computer resources, and one or two cases can often be completed within a day, as shown in Table 3-1. These models often do very well in predicting drag, lift, and turning moments on bodies in the flow that have relatively sharp edges that tend to fix the location of flow separation points as flow passes the body. Part of the reason they do well for these types of bodies is that the drag force is largely determined by the size and structure of the wake, and when the separation points are fixed by geometric features, the wake is very close to the correct size.

The RANS and URANS models are less accurate in predicting the flow and pressure around objects that have smoothly curving boundaries, because the point of separation is much more difficult to determine via numerical analysis. Small errors in the point and angle of flow separation from a body can lead to significantly larger errors in drag, lift, and turning moments. The difficulties in solving for the separation point and wake structure that develop from smoothly curved bodies like spheres, cylinders, and streamlined bodies are considerable. The static pressure drops and the dynamic pressure increases as the flow accelerates to move around the body. As the body cross section area decreases toward the rear, the flow decelerates as it moves through the growing cross section flow area, and there is a static pressure recovery in the expanding flow creating an adverse pressure that can result in flow separation from a smooth curved boundary. Predicting flow separation accurately over a smoothly curved body has the additional challenge of resolving the flow in the thin boundary layer at the body's surface requiring very dense computational grids and yet another difficulty predicting the transition from laminar to turbulent flow within the boundary layer, which can be challenging even when using LES turbulence models. The dimples on a golf ball introduce a small amount of surface roughness that triggers an earlier transition to turbulence in the boundary layer developing along the surface of the ball than that of a very smooth ball. The earlier transition to a turbulent boundary layer results in a delayed flow separation toward the rear of the ball, a smaller wake, greatly reduced drag, and a much farther flight distance, which is why golf balls are dimpled. Predicting this drag reduction with analysis requires predicting the point of transition to turbulence in the boundary layer in addition to the flow separation point, a task that is beyond the capabilities of basic RANS type turbulence models. In spite of these limitations, RANS turbulence models are widely used due to low cost and rapid turnaround times for cases that yield results that are good enough for engineering application.

The RANS turbulence model is recommended due to its low cost to run a case and fast completion time to reach stable convergence in cases that yield useful engineering results with the model. The stability of the convergence can be monitored by plotting the partial differential equation solver residuals. If they drop several orders of magnitude and become nearly steady with only small oscillations, then the case can be considered converged. Additional confidence in the convergence can be gained by also plotting the result quantities of interest, such as drag, lift, and turning moments. These quantities should go asymptotic with only very small oscillations in order to be 
considered converged. Generally, the RANS model will converge when the turbulent Reynolds number, defined above, for the body being analyzed is low enough for the mean flow to be stable (laminarized).

The URANS model is recommended when a stable converged solution cannot be obtained with the RANS model, also due to its low cost and fast completion time for a case. It generally requires only a small amount of additional computational resources and run time over those needed to run a RANS model case. The URANS model allows for unsteadiness in the flow field that varies, via eddy shedding or unsteady boundary conditions on a time scale that is long compared to the time scale of most of the turbulent fluctuations. Each time step is converged, and if a statistically steady state is sought, i.e. the boundary conditions are steady, the quantities of interest should be monitored with a moving average. When the moving average goes asymptotic, then the solution can be considered statistically steady, and both the time mean of the quantities of interest, such as drag, lift, and turning moments, and their deviation from the mean can be used for engineering applications. Design conditions, for example, may not be the time mean drag, but rather the time mean drag plus one or more deviations from the mean.

For the analysis of the aerodynamic response of very expensive structures or structures that are going to be used in very large quantities, large eddy simulation, LES, is recommended if the results of the RANS or URANS models are in doubt. Due to the large cost and time required to do an LES analysis, if engineering judgment is that LES analysis may be necessary, one of the cases can be run with LES and a much less expensive URANS analysis can also be run for comparison to determine if LES analysis is needed for the remainder of cases in a parametric case set. If differences in the quantities of interest are small, then LES is not needed.

\subsection{Recommendations for geometry models}

The geometry models tested included a full model with wind tunnel interior, fan, and exterior laboratory with contents, and several simplified models that did not include the interior of the wind tunnel, as presented in chapter 3 . Two fan models were tested, one with a rotating fan (see chapter 3.1) and one using a rotating reference frame model for turbo-machinery (see chapter 3.2) that imparts momentum to the air moving through the fan that results from moving blades but does not require mesh motion and sliding interfaces. Both of these models were used and can be used in the future to determine the velocity, pressure, and turbulence variable value distributions on a plane in the interior of the wind tunnel near its outlet, a location that can also be used as an inlet boundary to the test section.

Both of the full geometry wind tunnel laboratory models are expensive to run, and the rotating fan model is far more expensive to run than the rotating reference fan model. The rotating fan model was used to verify that the rotating reference frame model provided results that were good enough for engineering analysis and application (see chapter sections 4.1 and 4.2). The flow development moving down the wind tunnel interior through the screens and the return flow in the laboratory do have small impacts on the flow variable distribution at the upstream end of the test zone, and these small departures from a perfectly uniform approach flow for bodies in the test 
zone can affect some of the quantities of interest (drag, lift, turning moments) by more than a few percent.

Use of the rotating reference frame model is recommended if conditions that are very close to those of the actual laboratory at an upstream inlet to the test zone are needed for analysis. When CFD analysis is being used to extend results obtained from a case set tested in the physical wind tunnel to a larger case set, use of inlet boundary condition distributions that most closely match those of the laboratory is recommended. In addition, for case studies that are intended to extend laboratory results to a larger case set, once the test zone inlet boundary condition distributions are established, use of the simplified model (see chapter sections 3.3 and 4.3 ) that does not include the interior of the wind tunnel and has a flow inlet at the last screen is recommended.

The option to easily set perfectly uniform inlet boundary conditions using CFD analysis makes it possible and straightforward to test bodies in an ideal wind tunnel test zone. The test object of interest can be placed in a large box domain with uniform or any desired inlet boundary distributions for velocity, pressure, etc., and the sides top, bottom, and outlet can, for example, be set as pressure outlet boundaries. Varying angles of attack can be analyzed by changing all upwind sides of the domain to specified velocity inlets and specifying both the magnitude and direction of the velocity at those locations. A large variety of other test conditions can be created using a test box domain. This type of analysis is recommended as one that will be the least cost for running a parametric test matrix and provide the best results that can be obtained with CFD analysis for ideal test conditions.

Running CFD analysis at the full scale of the objects of study: bridge decks, road side hardware such as signage, etc., and other infrastructure components of interest, is also recommended. In physical wind tunnel laboratories, even the largest, running tests at full scale is rarely possible. For CFD aerodynamic analysis, running at full scale is generally no more work and does not require more computational resources as long as a larger range of flow structure size scales does not need to resolved in the computational mesh. Running tests at full scale eliminates any issues of scaling effects. 


\section{Acknowledgements}

The funding for this project came from the wind research program at Turner-Fairbank Highway Research Center, through Interagency Agreement Number DTFH61-14-X-300o02 between DOT and DOE, and the work was performed under DOE's contract with UChicago Argonne, LLC, contract no. DE-ACo2-O6-CH11357.

\section{References}

[1] https://www.solidworks.com/, last accessed July 2016

[2] CD-adapco, User Guide STAR-CCM+ version 11.02.010, 2016

[3] Lottes S.A., Bojanowski C., Shen J., 2013. Wind tunnel model of the Turner-Fairbank Highway Research Center Aerodynamics Laboratory, Argonne National Laboratory Report: ANL/ESD/13-3

[4] Bojanowski C., Lottes S., Sitek M., Zhang N., Bosch H., Modeling of wind flow in a realsize wind tunnel at FHWA Turner-Fairbank Aerodynamics Laboratory, 4th American Association for Wind Engineering Workshop, Miami, Florida, August 14-16, 2016

[5] Sitek M., Lottes S., Bojanowski C., Bosch H., 'Validation of CFD Models for a Virtual Aerodynamics Laboratory', 13th Americas Conference on Wind Engineering, Gainesville, FL, May 21-24, 2017

[6] McMaster-Carr, http://www.mcmaster.com/\#wire-mesh/=emqkhc, last accessed July 2016

[7] Mehta, R.D., 1985. Turbulent Boundary Layer Perturbed by a Screen, AIAA Journal, Vol. 2, No. 9, 1335-1342 


\section{Appendix A. Notes on wind tunnel modeling}

\section{A.1. Abbreviations}

To keep instructions for navigating and entering settings in the STAR-CCM+ user interface concise, the following abbreviations will be used:

Rclk = right click $\quad$ Lclk $=$ left click $\quad$ Dclk $=$ double click

Dlg $=$ dialogue box $\quad$ Pnl $=$ panel $\quad$ Pbox $=$ properties box

$\mathrm{TB}=$ tool bar $\quad$ Expl $=$ explorer $\quad+=$ expand

$\mathrm{A} \rightarrow \mathrm{B}=$ move down from $\mathrm{A}$ to $\mathrm{B}$ in object tree, expand $\mathrm{A}$ if needed

\section{A.2. Physical model design}

One of the base wind tunnel laboratory sim files with imported geometry is used for a new test. Open Star-CCM+ and choose the sim file.

\section{A.3. Preparing the domain for meshing}

Before meshing the domain, two modifications in the geometry will be made. First, a box in front of the wind tunnel outlet will be created, to assign a denser mesh to this region. It is highlighted in pink in Figure A.1.

+ Geometry $\rightarrow$ Rclk: Parts $\rightarrow$ New Shape Part $\rightarrow$ Block $\rightarrow$ Pbox:

Corner $1=[-0.95,2.7,-0.95] \mathrm{m}, \mathrm{m}, \mathrm{m}$

Corner $2=[0.95,0.0,0.95] \mathrm{m}, \mathrm{m}, \mathrm{m}$ 


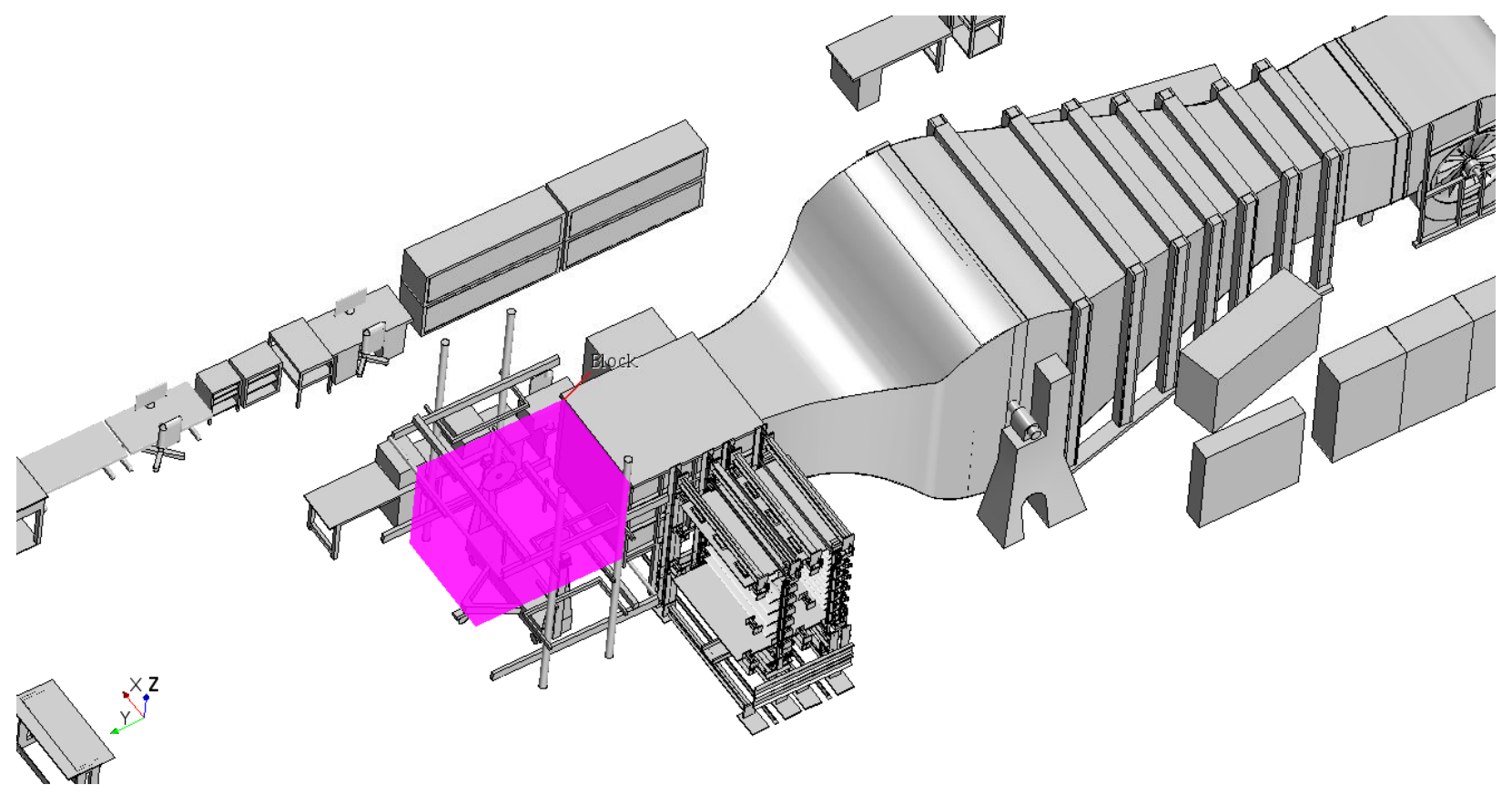

Figure A.1. A top view of the wind tunnel and the testing area

Second, a separate Region will be created in the outlet section of the wind tunnel to keep the meshing uniform inside of the tunnel. In order to do this, users need to "close" this section, by creating a new surface.

+ Geometry $\rightarrow$ Parts $\rightarrow$ AerodynamicLab $\rightarrow$ WTDE $77393 \rightarrow$ Rclk: Body $\rightarrow$ Repair Surface A new window will open with the chosen part ready to be edited.

Click on the central button with a line image on the bottom of the window. It will allow to choose only lines (not surfaces). Left click on the four lines with Shift on. The number of the chosen lines will be showed next to the line image. A screenshot of the main window with highlighted lines is shown in Figure A. 2. 


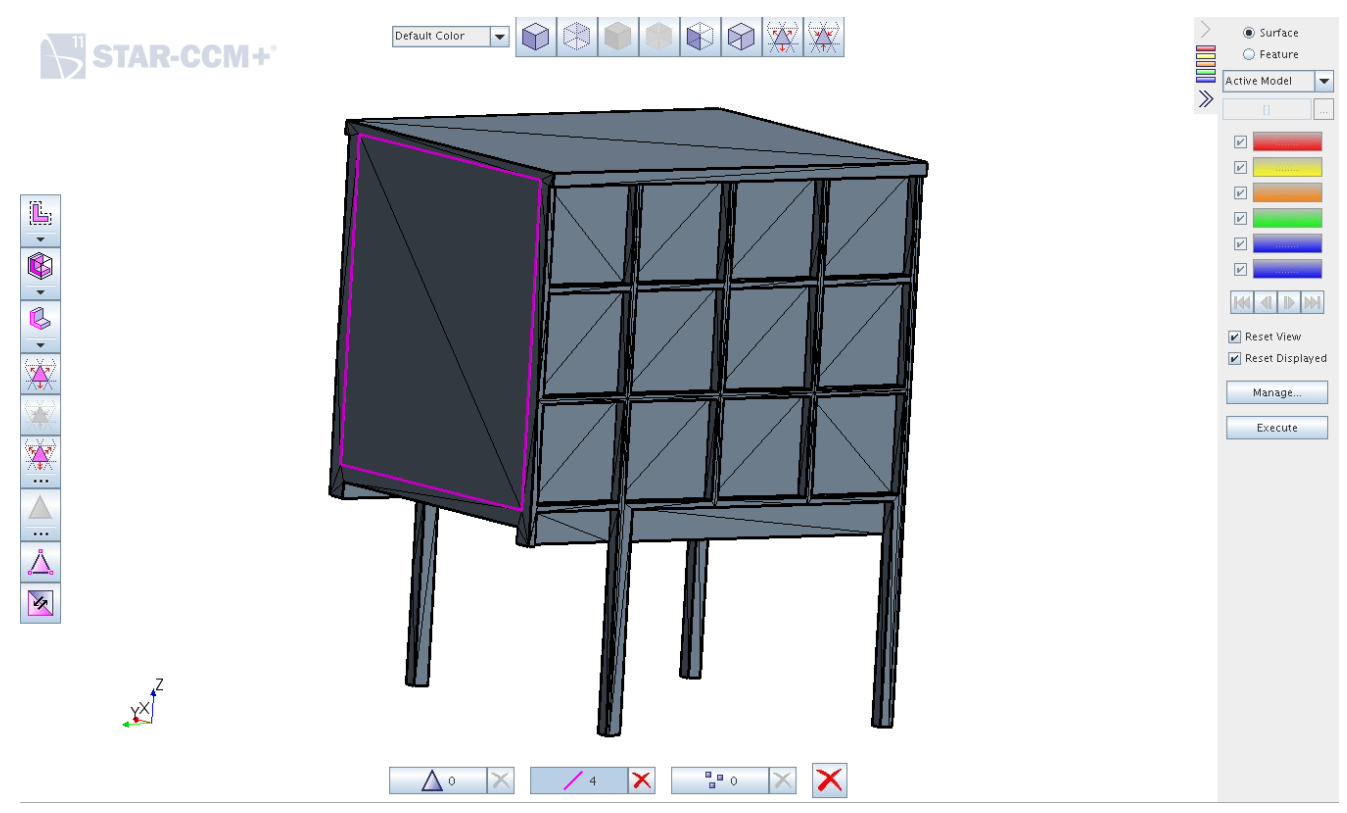

Figure A. 2. A screenshot of the main window with highlighted edges

In the Repair menu, click on the Fix Hole Using Selected Edges icon (it is the first one in the second line of Surface Repair set) Figure A.3.

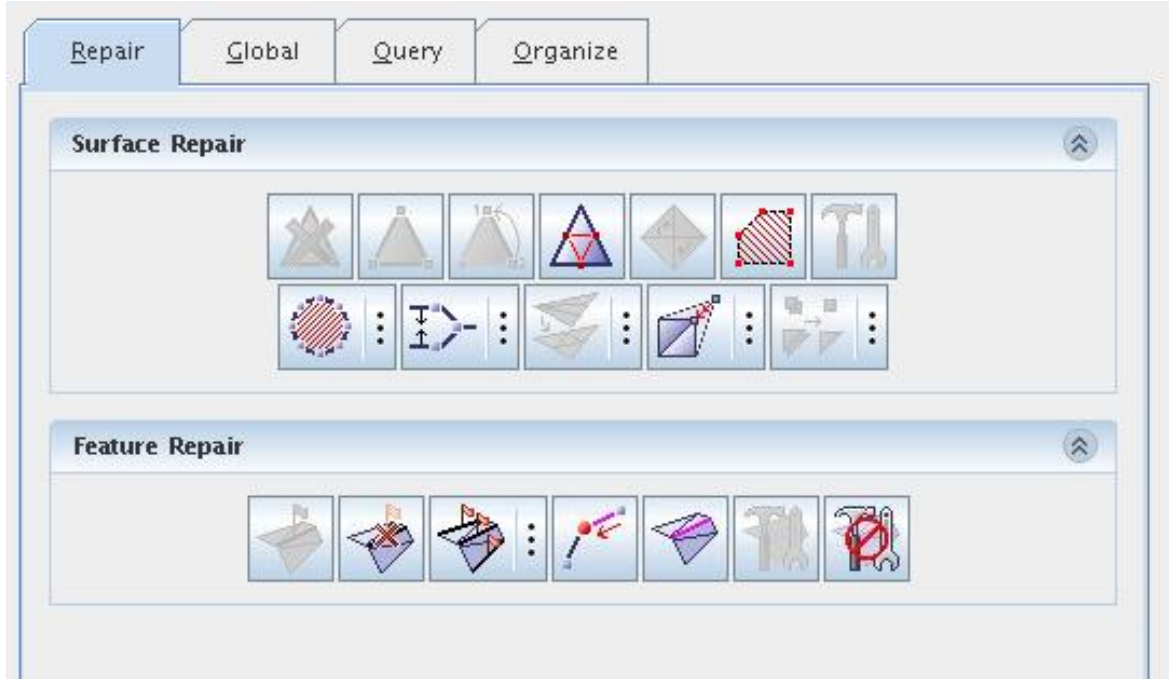

Figure A.3. The properties window with repair tools shown

The area in between the lines will be closed with a flat surface. 


\section{A.4. Using the Surface Wrapper}

The parts-based meshing option is used and the mesh is defined as a Geometry Operation. First, the user specified closed volumes are 'wrapped' using Surface Wrapper, then a mesh is assigned to the newly created volumes. The Surface Wrapper feature facilitates any mesh operation as it usually eliminates potential problems caused by imperfections of the geometry. The volumes created with the use of Surface Wrapper are: each section between screens inside of the wind tunnel (total of 10 sections), the rotating parts of the fan inside the cylinder, and the room.

To 'wrap' a volume, one needs to choose parts which surround it. For example, to create the wrapper in the room, all parts have to be checked. In this example the mounting frame is not included in the model, therefore the box on the left of "ModelMountingFrame" is empty (see Figure A. 4). The Wrapper will create a domain which consists of the space inside the room, minus the furniture, equipment and the wind tunnel. 


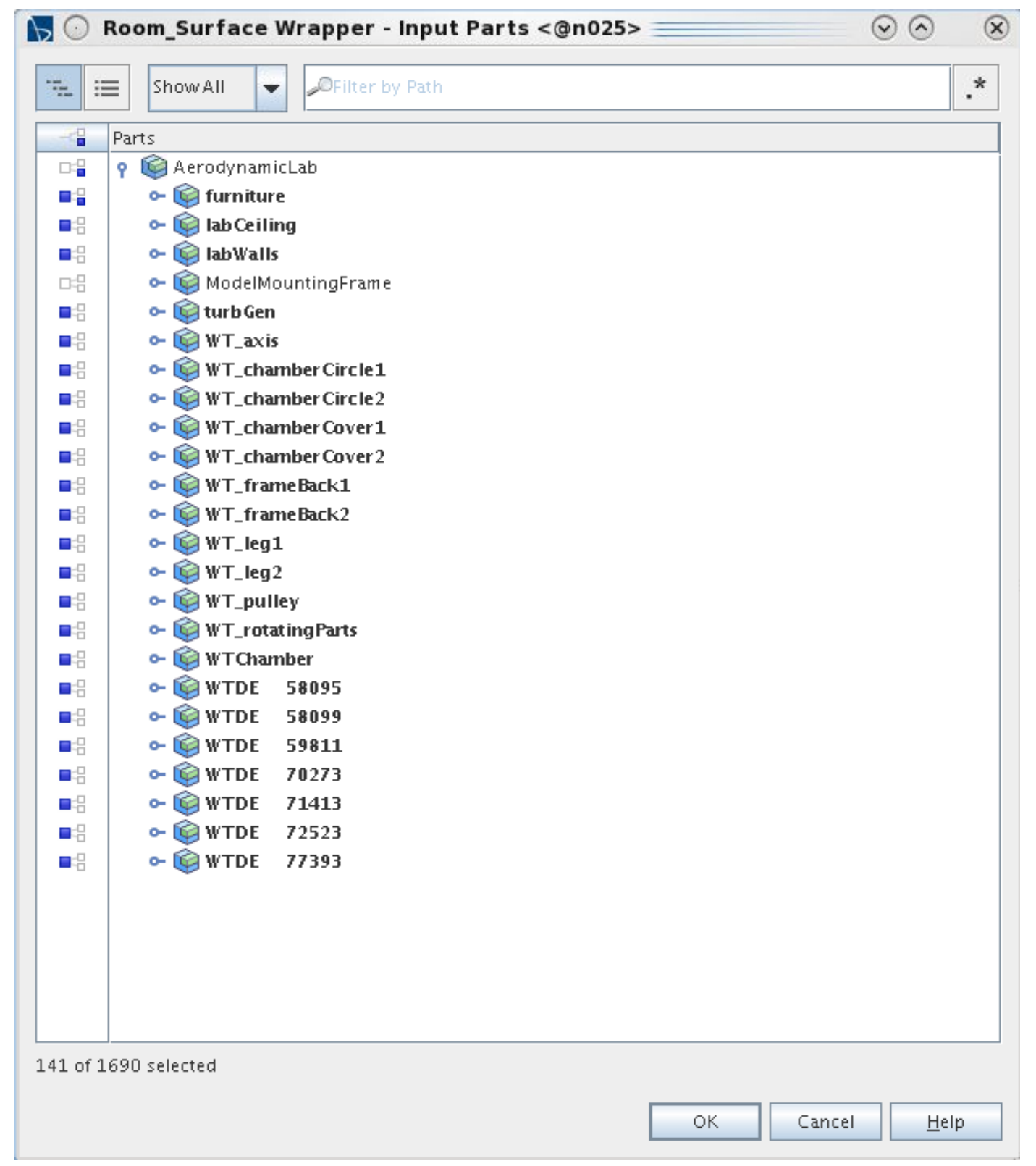

Figure A. 4. Parts chosen for the Room_Surface_Wrapper

A view of the external walls of the wrapped room is shown in Figure A. 5 and a view of the internal parts, with hidden wall and ceiling, is shown in Figure A. 6. 


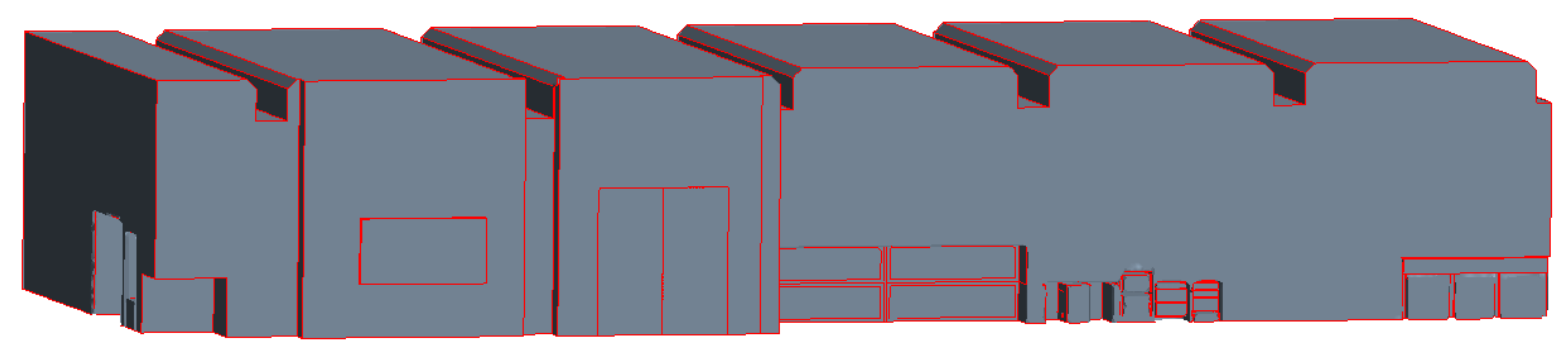

Figure A. 5. A side view of the domain after using the surface wrapper feature

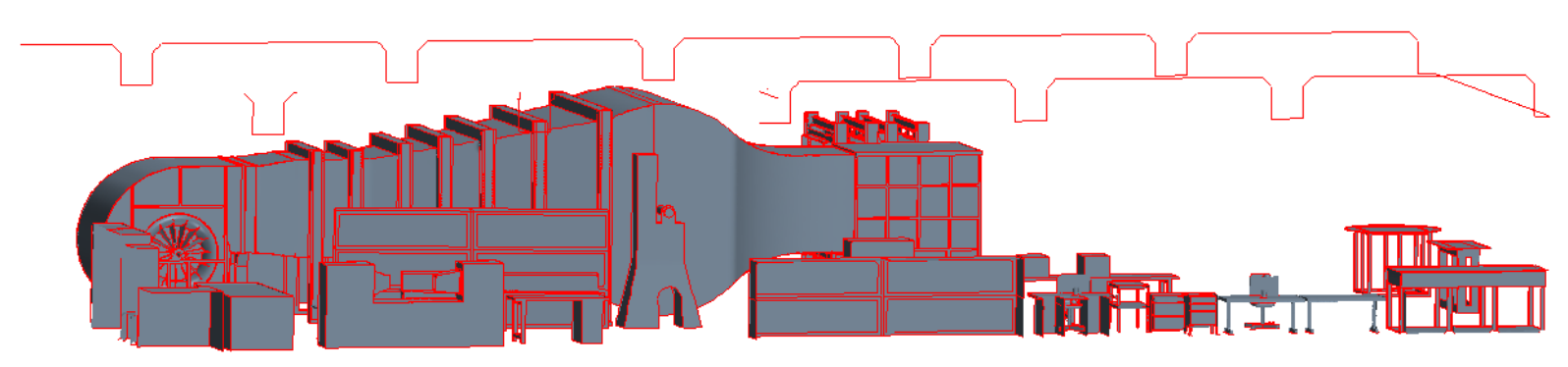

Figure A. 6. A view of the inside of the domain, with hidden walls and ceiling

Follow the steps below to create a Surface Wrapper for the room:

+ Geometry $\rightarrow$ Rclk: Operations $\rightarrow$ New $\rightarrow$ Surface Wrapper $\rightarrow$ Dlg: Parts $\rightarrow$ Check: furniture, labCeiling, ... (for the full list of parts, see the following figure) $\rightarrow$ OK

If the part is not selected you can always modify the selection under:

Operations $\rightarrow$ Lclk: Surface Wrapper $\rightarrow$ Pbox $\rightarrow$ Input Parts $\rightarrow[\ldots] \rightarrow$ Pbox $\rightarrow$ Select: ... $\rightarrow$ OK

Setup the parameters for the wrapper:

+ Default Controls $\rightarrow$ Base Size $\rightarrow$ Pbox $\rightarrow$ Value $=0.05 \mathrm{~m}$

+ Default Controls $\rightarrow$ Target Surface Size $\rightarrow$ Pbox $\rightarrow$ Relative Size $=100$

+ Default Controls $\rightarrow$ Minimum Surface Size $\rightarrow$ Pbox $\rightarrow$ Relative Size $=10$

Contact prevention is added to the Room_Surface Wrapper on the blades of the fan cover. Without it, the wrapper does not retain the edges of the blades and closes the gaps between them and the rotating fan.

Operations $\rightarrow$ Room_Surface Wrapper $\rightarrow$ Pbox $\rightarrow$ check: Perform Curvature Refinement

Operations $\rightarrow$ Room_Surface Wrapper $\rightarrow$ Surface Curvature $\rightarrow$ Basic Curvature $=80$ 
Operations $\rightarrow$ Room_Surface Wrapper $\rightarrow$ Pbox $\rightarrow$ check: Perform Gap Closure

Operations $\rightarrow$ Room_Surface Wrapper $\rightarrow$ Gap Closure $\rightarrow$ Gap Closure Size change to Absolute $\rightarrow$ Absolute Size $=0.01 \mathrm{~m}$

Operations $\rightarrow$ Rclk: Contact Prevention $\rightarrow$ One Group Contact Prevention Set $\rightarrow$ choose chamberCover1

Operations $\rightarrow$ Rclk: Contact Prevention $\rightarrow$ One Group Contact Prevention Set $\rightarrow$ choose chamberCover2

Operations $\rightarrow$ Rclk: Contact Prevention $\rightarrow$ Two Group Contact Prevention Set $\rightarrow$ choose chamberCover1 and rotatingParts

Operations $\rightarrow$ Rclk: Contact Prevention $\rightarrow$ Two Group Contact Prevention Set $\rightarrow$ choose chamberCover2 and rotatingParts

In the parts based meshing the wrapped surface becomes a new Geometry Part. To rename it, do the following:

+ Geometry $\rightarrow$ Parts $\rightarrow$ Rclk: Surface Wrapper $\rightarrow$ Rename $\rightarrow$ Dlg: New Name $=$ Room_Surface Wrapper $\rightarrow$ OK

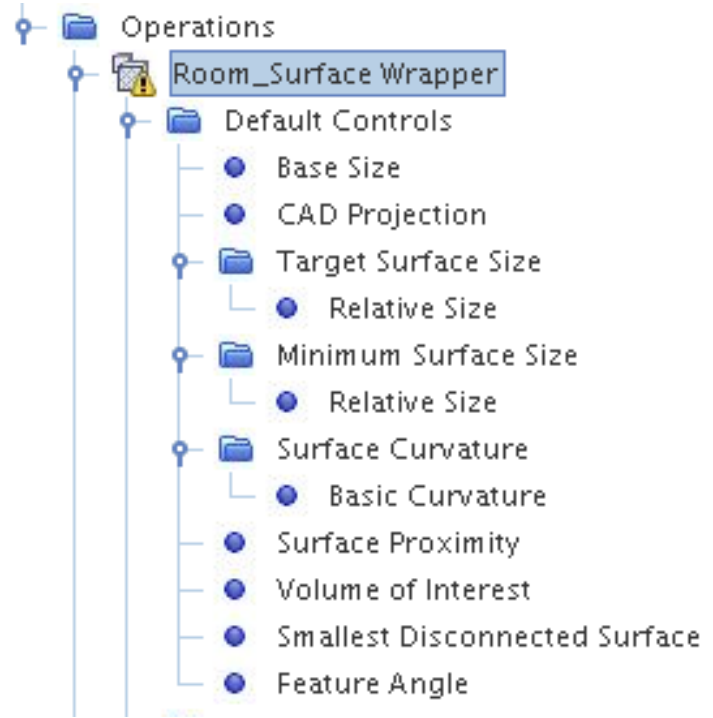

Figure A. 7. Part of the 'Operations' node in the model tree. Note the exclamation mark on the left of the Room_Surface_Wrapper

There is an exclamation sign in a yellow triangle on the icon of the newly created Surface Wrapper, as shown in Figure A. 7. This means that the wrapping is not yet performed. To execute the operation, do the following:

+ Operations $\rightarrow$ Rclk: Surface Wrapper $\rightarrow$ Execute 
The exclamation sign disappears when the wrapping operation is complete and the model tree looks like in Figure A.8.

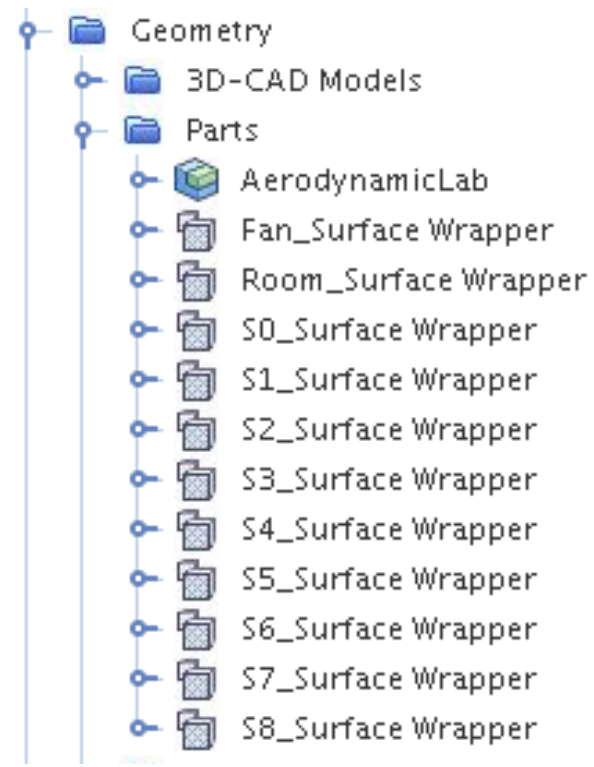

Figure A.8. Part of the 'Operations' node in the model tree after wrapping is performed.

Fan_Surface Wrapper:

+ Operations $\rightarrow$ Fan_Surface Wrapper $\rightarrow$ Default Controls $\rightarrow$ Base Size $\rightarrow$ Pbox $\rightarrow$ Value $=$ 0.01m

+ Default Controls $\rightarrow$ Target Surface Size $\rightarrow$ Pbox $\rightarrow$ Relative Size $=80$

+ Default Controls $\rightarrow$ Minimum Surface Size $\rightarrow$ Pbox $\rightarrow$ Relative Size $=10$

+ Default Controls $\rightarrow$ Pbox $\rightarrow$ check: Perform Curvature Refinement

+ Default Controls $\rightarrow$ Surface Curvature $\rightarrow$ Basic Curvature $=180$

So_Surface Wrapper:

Operations $\rightarrow$ So_Surface Wrapper $\rightarrow$ Default Controls $\rightarrow$ Base Size $\rightarrow$ Pbox $\rightarrow$ Value $=$ 0.05m

+ Default Controls $\rightarrow$ Target Surface Size $\rightarrow$ Pbox $\rightarrow$ Relative Size $=100$

+ Default Controls $\rightarrow$ Minimum Surface Size $\rightarrow$ Pbox $\rightarrow$ Relative Size $=25$

+ Default Controls $\rightarrow$ Pbox $\rightarrow$ check: Perform Curvature Refinement

+ Default Controls $\rightarrow$ Surface Curvature $\rightarrow$ Basic Curvature $=180$

Sections S1 to S8 have the same wrapper properties:

+ Operations $\rightarrow$ S1_Surface Wrapper $\rightarrow$ Default Controls $\rightarrow$ Base Size $\rightarrow$ Pbox $\rightarrow$ Value $=0.1 \mathrm{~m}$ 
+ Default Controls $\rightarrow$ Target Surface Size $\rightarrow$ Pbox $\rightarrow$ Relative Size $=100$

+ Default Controls $\rightarrow$ Minimum Surface Size $\rightarrow$ Pbox $\rightarrow$ Relative Size $=10$

+ Default Controls $\rightarrow$ Pbox $\rightarrow$ check: Perform Curvature Refinement

+ Default Controls $\rightarrow$ Surface Curvature $\rightarrow$ Basic Curvature $=36$

The number of number of points/circle are increased for Fan_Surface Wrapper and So_Surface Wrapper, because the interface between these two regions has cylindrical shape. In finite volume software a circle is always represented by a polygon. It is important to choose an appropriate number of polygon edges, so that the approximation is good enough. In this model the default number of 36 points/circle causes a deformation of the first layer of cells in So_Surface Wrapper when the cylinder (with a polygonal, not circular base) is rotating.

The advantage of Surface Wrapper meshing technique is that the user has an easy access to the wrapper settings and volume mesh settings. All the curve, surface or volume based custom settings are located in the Operations node. There is no need to go to Regions to modify each surface separately, as is required with region based meshing. Note that additional surface and volume controls can be added to use more refined settings on specific surfaces of a part or portions of the volume enclosed by the part.

Before a volume mesh is created, the wrapped part needs to be assigned to a region. This is done in the following steps:

Rclk: Room_Surface Wrapper $\rightarrow$ Assign Parts to Regions... $\rightarrow$ Dlg: Create One Region for All Parts = Room, Change selection from 'Create a Boundary for Each Part' to 'Create a Boundary for Each Part Surface' $\rightarrow$ Apply $\rightarrow$ Close

\section{A.6. Defining boundary conditions}

In this model all laboratory walls, ceiling, floors, all furniture and equipment present in the room are modeled as wall boundary conditions. The wind tunnel screens are modeled as porous baffle interfaces between regions. Three internal interfaces are also created for the boundaries between the rotating fan and the room as well as the inside of the wind tunnel. Interfaces numbered from 1 to 9 are Porous Baffle Interfaces and Interfaces 10 to 12 are Internal Interfaces. The Interfaces node, with all mentioned interfaces, is shown in Figure A.9. Figure A. 10 shows all Porous Baffle Interfaces and Figure A. 11 shows all Internal Interfaces present in the model. 


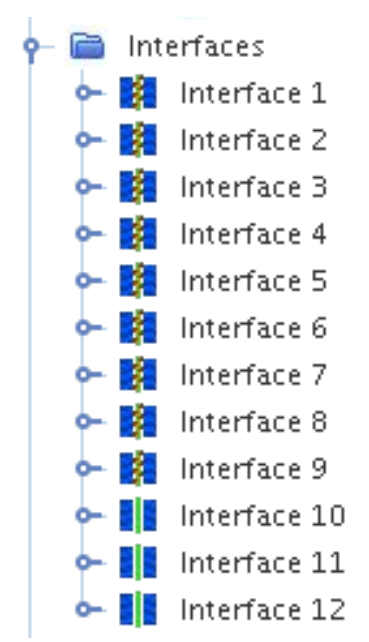

Figure A.9. The Interfaces node in the model tree

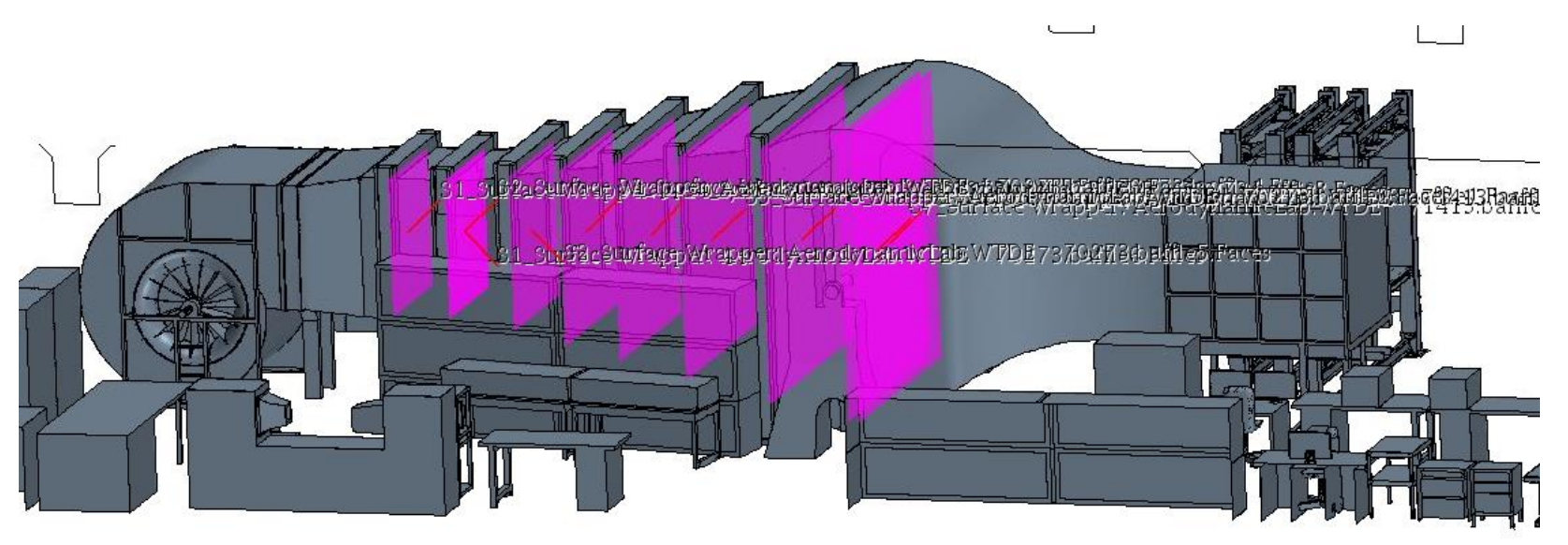

Figure A. 10. The wind tunnel with Porous Baffle Interfaces highlighted in pink

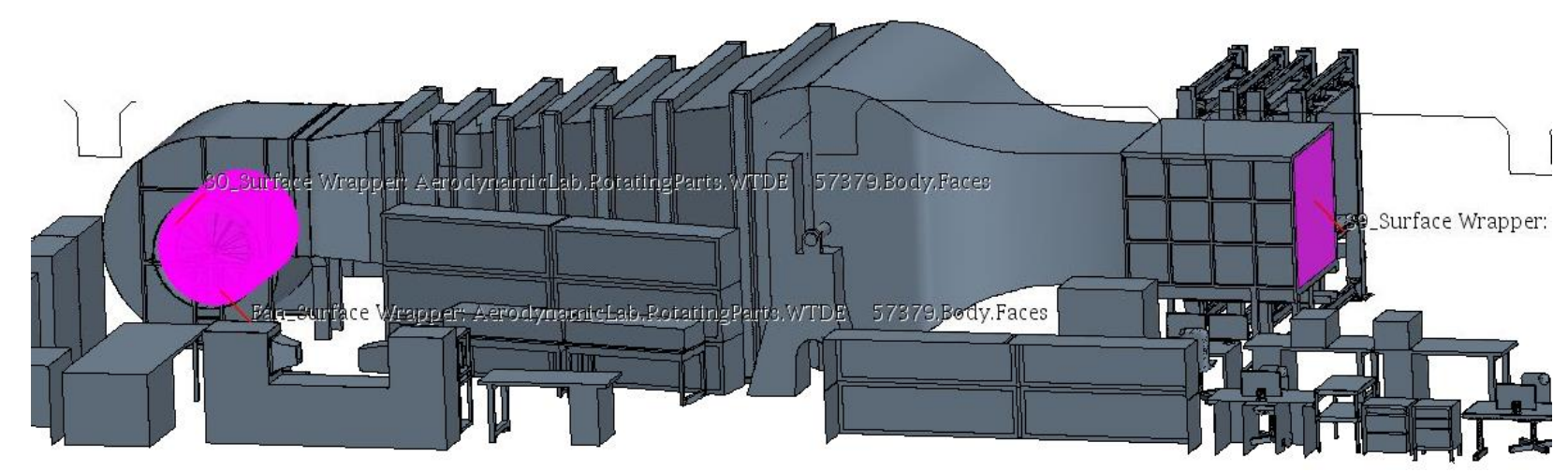

Figure A. 11. The wind tunnel with Internal Interfaces highlighted in pink 
To create an interface the user needs to choose corresponding surfaces in the Regions nodes. E.g. to create an internal interface between the Room_Surface Wrapper and Fan_Surface Wrapper do the following:

+ Regions $\rightarrow$ Room_Surface Wrapper $\rightarrow$ Boundaries $\rightarrow$ Lck:

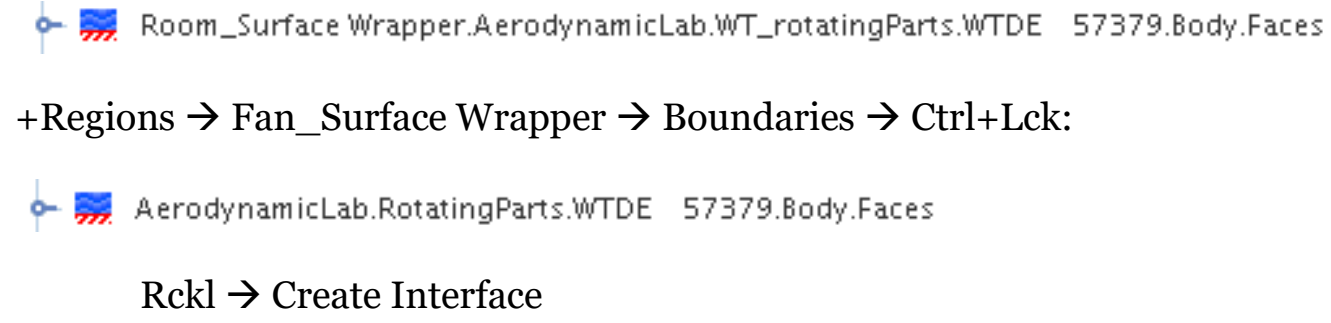

A new node, called Interfaces, appears in the main object tree on the left. By default an Internal Interface is created and no changes have to be made in the properties window.

If a porous baffle interface is required between the surfaces, as in the case of the mesh screens inside of the wind tunnel, the properties of the interface must be changed as following:

+ Interfaces $\rightarrow$ Interface $1 \rightarrow$ Pbox: change Type to Porous Baffle Interface

This type of interface has three parameters: Porosity, Porous Inertial Resistance and Porous Viscous Resistance. Set up these properties:

+ Interface $\rightarrow$ Interface $1 \rightarrow$ Physics Values $\rightarrow$ Porosity $=0.672$

Porous Inertial Resistance and Porous Viscous Resistance are by default equal zero. In this example the porous inertial resistance is assigned a field function, and the porous viscous resistance is equal zero. Additional steps are required to create a field function. First, create a report

Lck: Reports $\rightarrow$ New $\rightarrow$ Average

Scalar Field = Velocity[j]

Parts $=$ Interface 1

Change the name of the report to vel_ave_b1. Repeat for the rest of the screens. Set Porous Inertial Resistance value to:

Porous Inertial Resistance $=\$\{$ vel_ave_b1Report $\}<0.01$ ? $0.1: 2.4 * \operatorname{pow}((\$\{$ vel_ave_b1Report $\}$

* 17.9), (-1.0/3.0) )

\section{A.7. Volume mesh and meshing}

To define the volume mesh settings: 
+ Geometry $\rightarrow$ Rclk: Operations $\rightarrow$ New $\rightarrow$ Automated Mesh $\rightarrow$ Select Room_Surface Wrapper in Parts Box $\rightarrow$ in Select Meshers box select: Surface Remesher, Polyhedral Mesher, Prism Layer Mesher $\rightarrow$ OK

Now, modify the default Reference Values for the volume meshing process:

Automated Mesh $\rightarrow$ Default Controls $\rightarrow$ Base Size $\rightarrow$ Pbox $\rightarrow$ Value $=0.1 \mathrm{~m}$

Default Controls $\rightarrow$ Prism Layer Total Thickness $\rightarrow$ Pbox $\rightarrow$ Size type: Absolute

Absolute Size $\rightarrow$ Pbox $\rightarrow$ Value $=0.05 \mathrm{~m}$

To assign different meshing controls to the previously created box in front of the wind tunnel outlet, do the following:

+ Geometry $\rightarrow$ Operations $\rightarrow$ Room_Automated Mesh $\rightarrow$ Rckl: Custom Controls $\rightarrow$ New: Volumetric Control

Part $=$ Box

Controls $\rightarrow$ Polyhedral Mesher: check the box

Values $\rightarrow$ Rckl: Custom Size $\rightarrow$ Absolute Size $\rightarrow$ Value $=0.05 \mathrm{~m}$

Set up the base size for the remaining regions. To create the mesh with the chosen parameters, do the following:

Rclk: Automated Mesh $\rightarrow$ Execute

\section{A.8. Defining physics}

The physics for this model are as following:

+Continua $\rightarrow$ Physics $1 \rightarrow$ Rclk: Models $\rightarrow$ Select Models... $\rightarrow$ Dlg

Select: Three Dimensional, Steady, Gas, Segregated Flow, Constant Density, Turbulent, K-Epsilon Turbulence $\rightarrow$ Close

\section{A.9. Defining fan rotation}

The rotation of the fan will be simulated with the use of a rotating reference frame. To define the axis of rotation, find a point laying on it. One way to do it is to use the center of gravity of the part WT_axis.

+ Parts $\rightarrow$ Rckl: WT_axis $\rightarrow$ Edit CAD

In the right window $3 \mathrm{D}-\mathrm{CAD}$ View will open with a view of the part in question. To find the properties of the body, go to: 
+Bodies $\rightarrow$ Rckl: Body $1 \rightarrow$ Properties

Body properties, such as volume, center of gravity and moments of inertia, are displayed in the Output window (on the bottom of the screen), as shown in Figure A. 12.

\begin{tabular}{|c|c|}
\hline Body Name: & Body 1 \\
\hline $\begin{array}{l}\text { Volume: } \\
\text { Center of Gravity: } \\
\text { Moment of Inertia: }\end{array}$ & $\begin{array}{l}0.030053 \mathrm{~m} / 3 \\
{[0.053832,-15.589,-0.83503] \mathrm{m}, \mathrm{m}, \mathrm{m}} \\
\text { Ix: }[5.5871 \mathrm{e}-05,-2.6446 \mathrm{e}-13,5.2954 \mathrm{e}-13] \mathrm{kg}-\mathrm{m} / 2, \mathrm{~kg}-\mathrm{m} / 2, \mathrm{~kg}-\mathrm{m} / 2 \\
\text { Iy: }[-2.6446 \mathrm{e}-13,0.015175,-1.7110 \mathrm{e}-09] \mathrm{kg}-\mathrm{m} / 2, \mathrm{~kg}-\mathrm{m} \wedge 2, \mathrm{~kg}-\mathrm{m} / 2 \\
\text { Iz: }[5.2954 \mathrm{e}-13,-1.7110 \mathrm{e}-09,0.015175] \mathrm{kg}-\mathrm{m} / 2, \mathrm{~kg}-\mathrm{m} \wedge 2, \mathrm{~kg}-\mathrm{m} / 2\end{array}$ \\
\hline
\end{tabular}

Figure A. 12. The output window showing the properties of the WT_axis part

To define a rotating reference frame do the following:

+Tools $\rightarrow$ Rclk: Reference Frames $\rightarrow$ New: Rotation

Rotation $\rightarrow$ Axis Direction $=[1.0,0.0,0.0]$

Rotation $\rightarrow$ Axis Origin $=[0.053832,-15.589,-0.83503] \mathrm{m}$

Rotation $\rightarrow$ Rotation Rate $=-437.0$ rpm (or other desired rate)

To assign the rotation to the fan region, go to:

+ Region $\rightarrow$ Fan $\rightarrow$ Physics Values $\rightarrow$ Motion Specification $\rightarrow$ Reference Frame $=$ Rotating

\section{A.10. Solver settings}

Set the required number of iterations in the Steady Solver.

+ Stopping Criteria $\rightarrow$ Maximum Steps $\rightarrow$ Pbox: Maximum Steps $=10000$ (or other desired value)

To run a job click the "running man" icon

\section{A.11. Introducing a test model to the wind tunnel domain}

There are multiple methods in Star-CCM+ to introduce a selected test model, such as a bridge section model or a model of roadside hardware, to the wind tunnel domain and perform simulations of the laboratory experiments.

One approach is to create an additional part in the wind tunnel domain, by importing the geometry of the test model as surface mesh. The new part must be added to the set of parts used in the Room_Surface_Wrapper.Surface wrapping and meshing of the entire domain, with the 
additional part inside, has to be performed. As the model has a complex geometry, it takes a significant amount of time.

In another approach, the test model is first enclosed in a box domain, outside of the wind tunnel domain. Surface Wrapper is used to create a new geometry, which is assigned to a region and meshing is done. At the same time, the aerodynamic laboratory domain is modified by introducing a box part of the same dimensions in the location where the box with the test model is supposed to be positioned. This box is excluded from the room region, by wrapping around it. Then, the new box region is imported to the wind tunnel model and the common surfaces between the two regions are changed into internal interfaces. This method avoids remeshing of the wind tunnel model every time a new box region with a test model inside is introduced, as long as the location and dimensions of the box region do not change. If the user wants to make any changes to the box dimensions, the whole process has to be repeated.

The recommended approach is to use a feature called Overset Mesh. In this method an additional box region with the test model inside has to be created and meshing needs to be performed. Then the region is imported to the wind tunnel domain as a Volume Mesh, but instead of Internal Interfaces, as it was done in the previously described method, an Overset Mesh Interface is created. This way makes it possible to merge the two models without any remeshing necessary. The position of the box domain can be modified, or imported again with different dimensions and the wind tunnel domain will not be affected. The following steps show how to use this approach.

Prepare the geometry of a box domain with a test model inside. Assign a region to the part and chosen mesh settings. After meshing is done, export the Volume Mesh as a *.ccm file:

File $\rightarrow$ Export

leave the default settings in the pop-up window and click Save button

Open the wind tunnel model and import the newly created volume mesh:

File $\rightarrow$ Import $\rightarrow$ Import Volume Mesh, choose the *.ccm file

The volume mesh is imported as a new region. It can be seen as a new line in the Regions node. To create an Overset Mesh interface between the two regions, do the following:

+ Regions $\rightarrow$ Rcklk on both regions $\rightarrow$ Create Interface $\rightarrow$ Overset Mesh

New node is created in Interfaces, called Overset Mesh 1. The boundary conditions on the box region surfaces need to be changed to Overset Mesh:

+ Regions $\rightarrow$ Boundaries $\rightarrow$ Lcklk on a boundary $\rightarrow$ Properties: Type $\rightarrow$ Overset Mesh 


\section{Appendix B.}

This appendix provides the details of flow approaching the test zone coming from the wind tunnel with no test object mounted in the force balance. The flow was computed using CFD with three CFD models: a model with rotating reference frame, a simplified geometry model with uniform inlet variables and a model with non-uniform inlet variables using RANS solvers. Variables plotted include velocity magnitude, velocity component values, turbulence intensity, and turbulent viscosity. Among other things, the results of the analysis show the variation of these quantities that can be expected across the approach flow to the test zone. These cases use inlet boundary conditions with uniform flow variable values entering the domain as the flow leaves the last screen of the wind tunnel. In reality, there is also some variation over the last screen, and therefore, the actual deviations from uniform in the approach flow to the test zone would be expected to be a little larger than those shown in the plots in this appendix.

Figure B. 1 shows the velocity magnitude distribution on a horizontal plane in the testing zone and the extension of the wind tunnel, as well as the geometry of the force balance and the wind tunnel. A vertical plane, located $60 \mathrm{~cm}$ away from the extension, is marked with a dashed line. A detailed analysis of the velocity components was performed for points on this plane, with horizontal and vertical coordinates ranging from -0.9 meters to 0.9 meters (with the CSYS origin in the center of the outlet surface of the wind tunnel extension).

Figure B. 2 shows velocity magnitude on a vertical plane, located $60 \mathrm{~cm}$ away from the wind tunnel extension, at fan speed $48 \mathrm{rpm}$, (a) with rotating reference frame, (b) simplified geometry with uniform inlet velocity, (c) simplified geometry with non-uniform inlet velocity. The next three figures, from Figure B. 3 to Figure B. 5, present plots of the X, Y, and Z velocity components. Turbulence intensity is shown in Figure B. 6, and turbulent viscosity ratio in Figure B. 7. Plots in Figure B. 8 to Figure B. 13 illustrate the same set of results at fan speed $437 \mathrm{rpm}$.

Also, the direction of the velocity vector was compared for the three models. Angles between the vector and Y axis on XY plane and YZ plane were combined in Figure B. 14 to Figure B. 17. The angles were calculated from the known velocity components, as

$$
\begin{aligned}
& \operatorname{Angle}_{X Y}=\operatorname{atan}\left(\frac{v_{X}}{v_{Y}}\right), \\
& \operatorname{Angle}_{Y Z}=\operatorname{atan}\left(\frac{v_{Z}}{v_{Y}}\right)
\end{aligned}
$$

where $v_{X}, v_{Y}$, and $v_{Z}$ are the velocity components in $\mathrm{X}, \mathrm{Y}$ and $\mathrm{Z}$ direction respectively.

Unfortunately, no laboratory measurements of velocity direction were available for the considered plane, nor the testing zone. The only measurements were done for areas outside of the volume of interest. History plots of velocity direction for two points, number 20 and 23, were presented in Figure 2-9 and Figure 2-11 and the time averaged values in Table 2-1 and Table 2-2. These two points are in the downstream area with respect to the testing zone, therefore it can be assumed that the angle between the velocity vector and Y axis is higher there than in the testing zone. Taking this into account, the expected time averaged angle in the testing zone should be less than 
5 degrees. Out of the considered models, only the model with simplified geometry and uniform velocity and turbulence parameters at the inlet boundary condition, satisfies this criterion. The time averaged absolute value of the angle is less than 5 degrees for the whole set of probes. In opposite to the laboratory measurements, this model does not show oscillations of velocity magnitude or direction. Both quantities are constant in the simulation. In the other two models, the absolute value of the angle can be as high as 16 degrees.

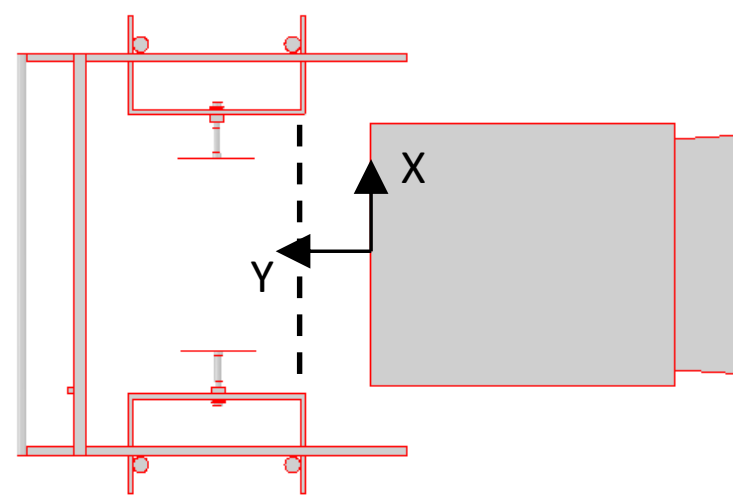

Figure B. 1. . The geometry of the force balance located in the testing zone the extension of the wind tunnel are is shown. A vertical plane, located $60 \mathrm{~cm}$ away from the extension, is marked with a dashed line.

(a)

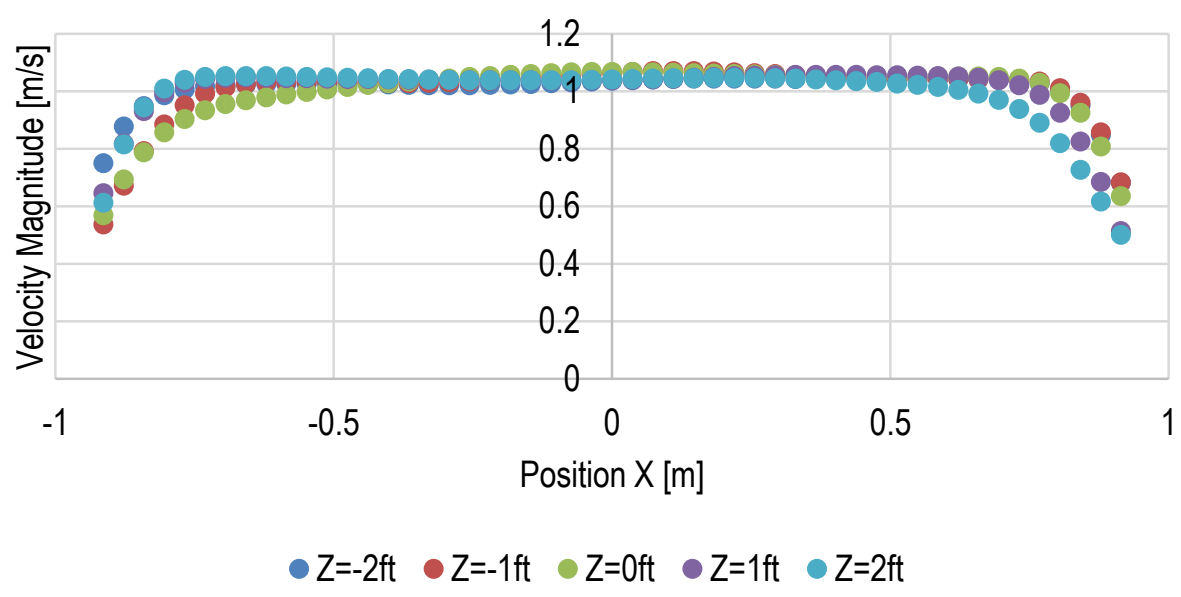


(b)

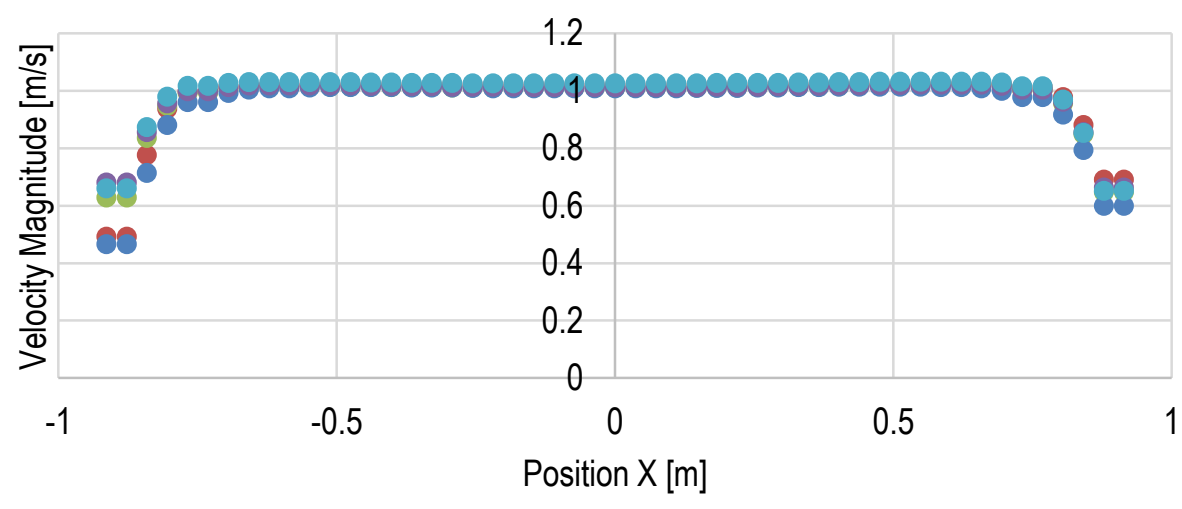

$$
\bullet Z=-2 \mathrm{ft} \odot Z=-1 \mathrm{ft} \odot Z=0 \mathrm{ft} \cdot Z=1 \mathrm{ft} \odot Z=2 \mathrm{ft}
$$

(c)

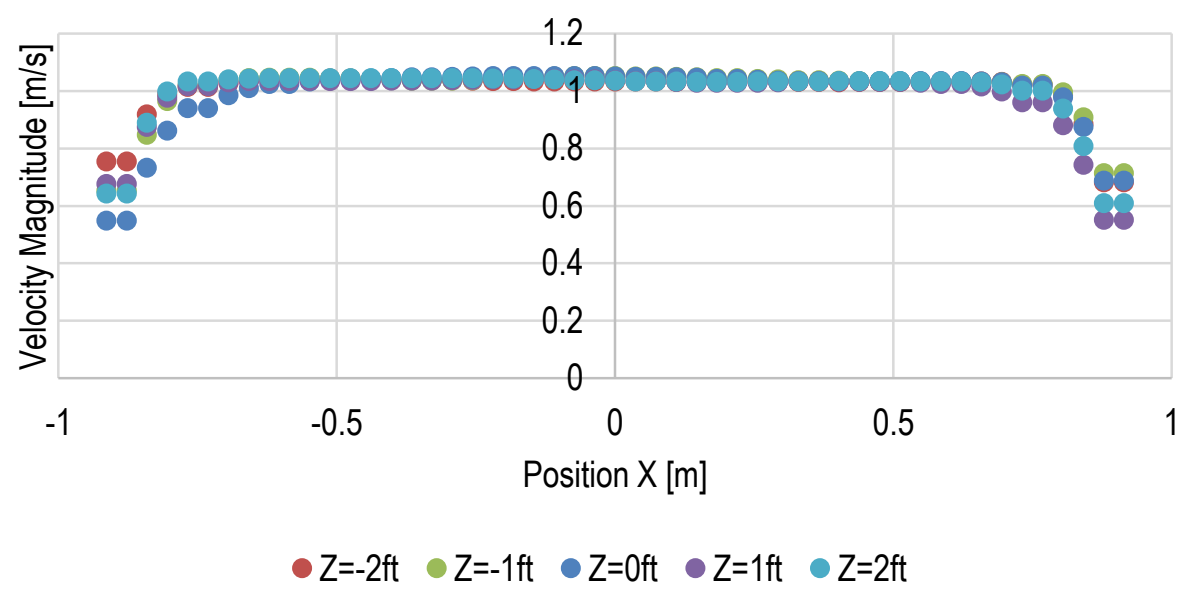

Figure B. 2. Velocity magnitude on a vertical plane, located half way between the centerline between the force balance plates and the outlet of the wind tunnel nozzle, at fan speed $48 \mathrm{rpm}$, (a) with rotating reference frame, (b) simplified geometry with uniform inlet velocity, (c) simplified geometry with nonuniform inlet velocity 
(a)

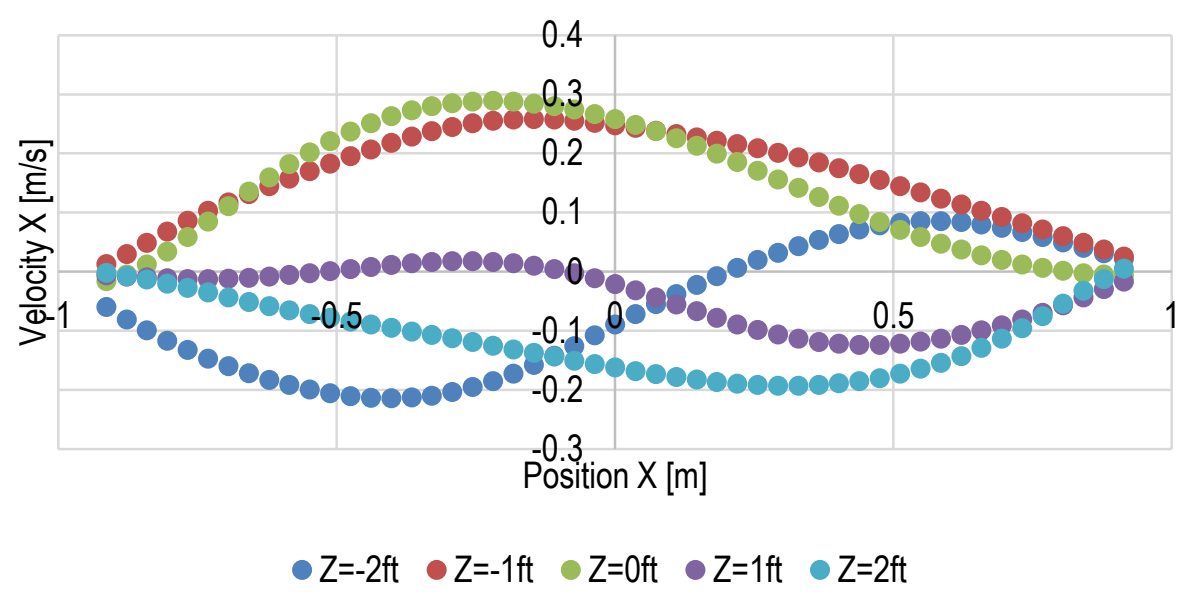

(b)

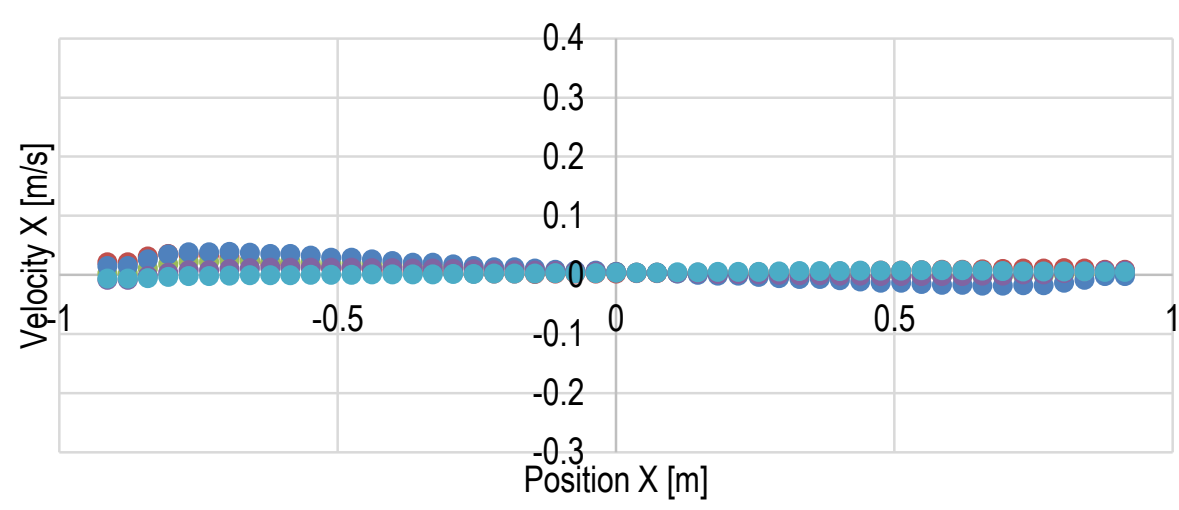

$\bullet Z=-2 f t \quad Z=-1 \mathrm{ft} \bullet Z=0 \mathrm{ft} \quad \mathrm{Z}=1 \mathrm{ft} \bullet \mathrm{Z}=2 \mathrm{ft}$

(c)

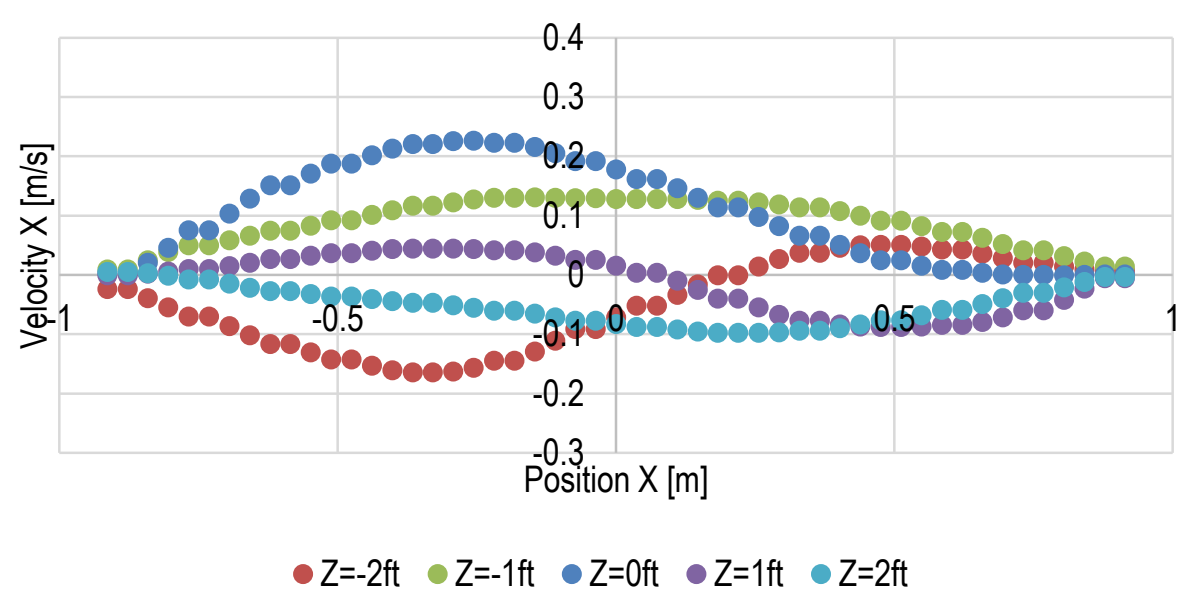

Figure B. 3. Velocity in X direction on a vertical plane, located half way between the centerline between the force balance plates and the outlet of the wind tunnel nozzle, at fan speed $48 \mathrm{rpm}$, (a) with rotating reference frame, (b) simplified geometry with uniform inlet velocity, (c) simplified geometry with nonuniform inlet velocity 
(a)

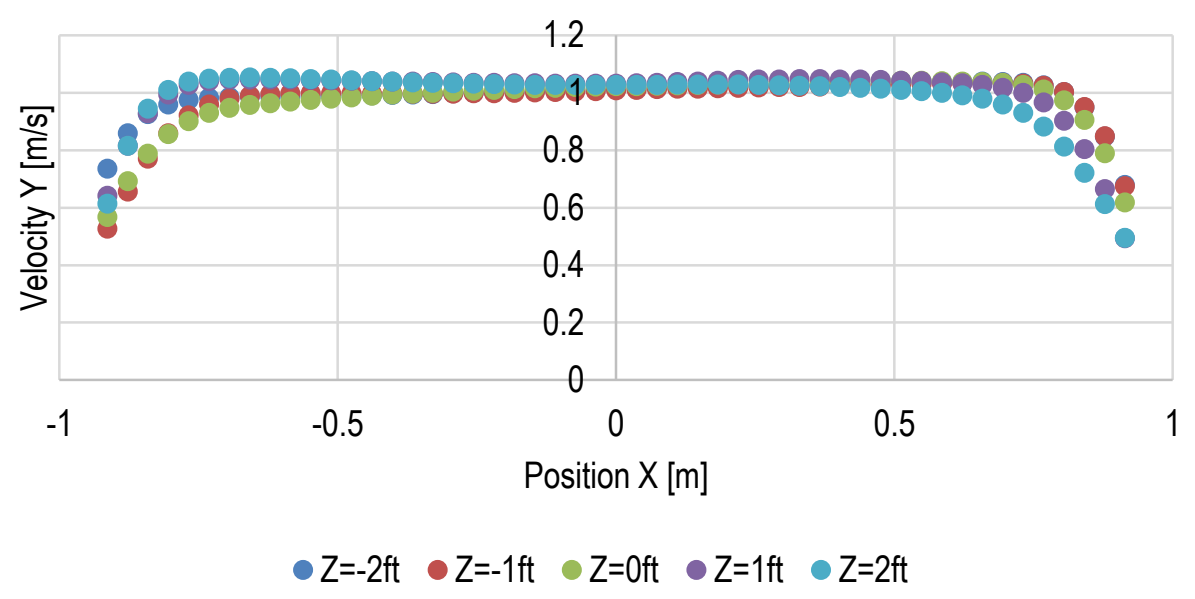

(b)

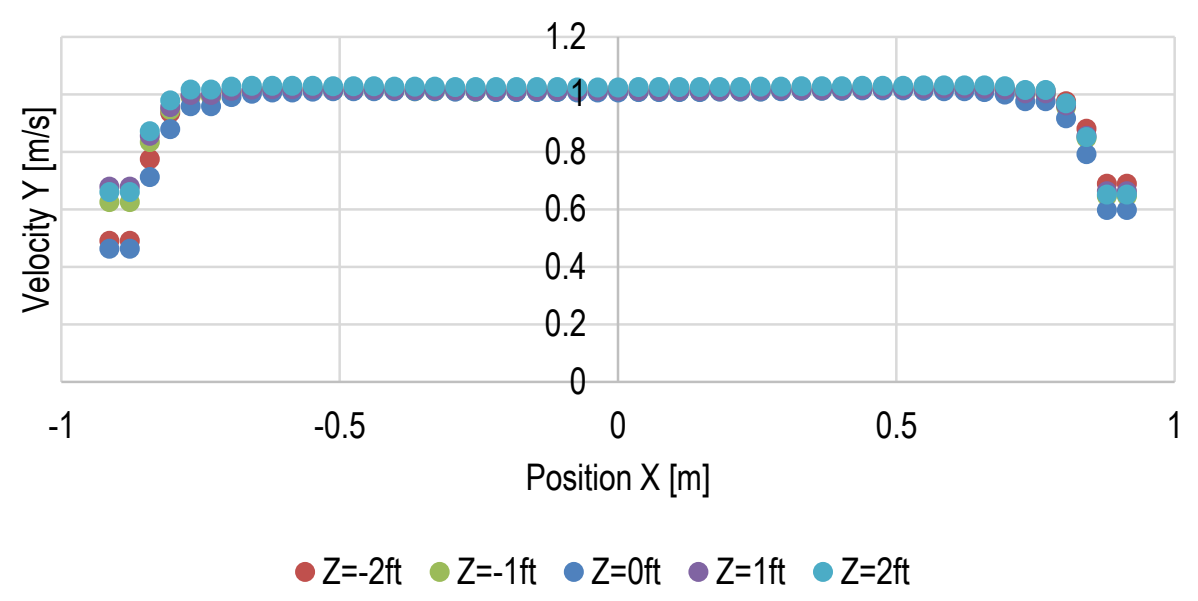

(c)

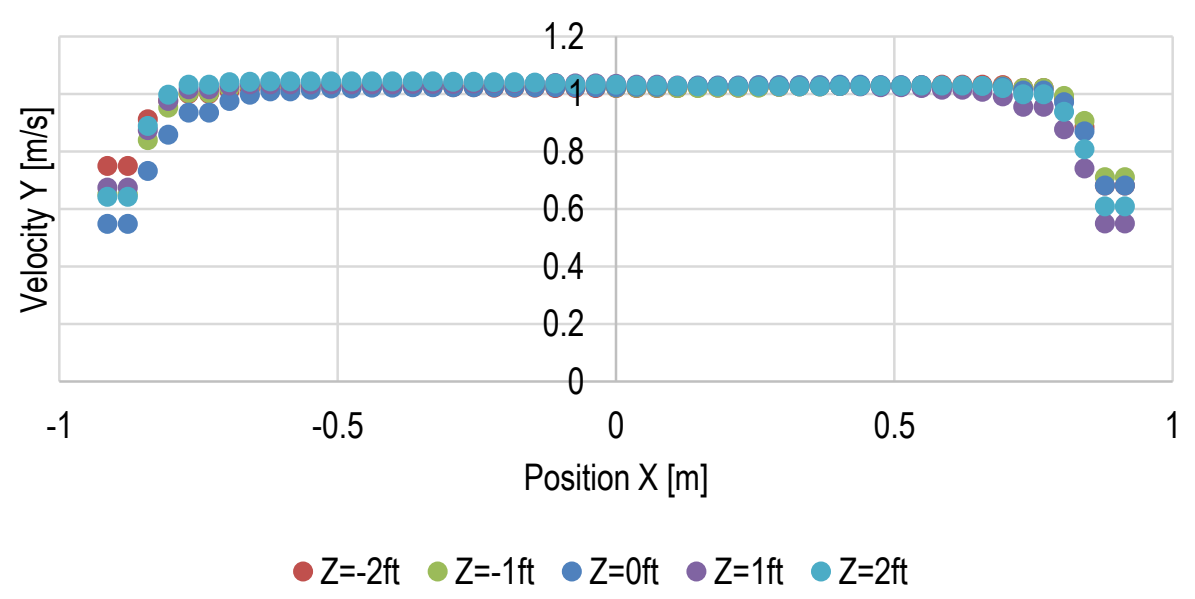

Figure B. 4. Velocity in Y direction on a vertical plane, located half way between the centerline between the force balance plates and the outlet of the wind tunnel nozzle, at fan speed $48 \mathrm{rpm}$, (a) with rotating reference frame, (b) simplified geometry with uniform inlet velocity, (c) simplified geometry with nonuniform inlet 
(a)

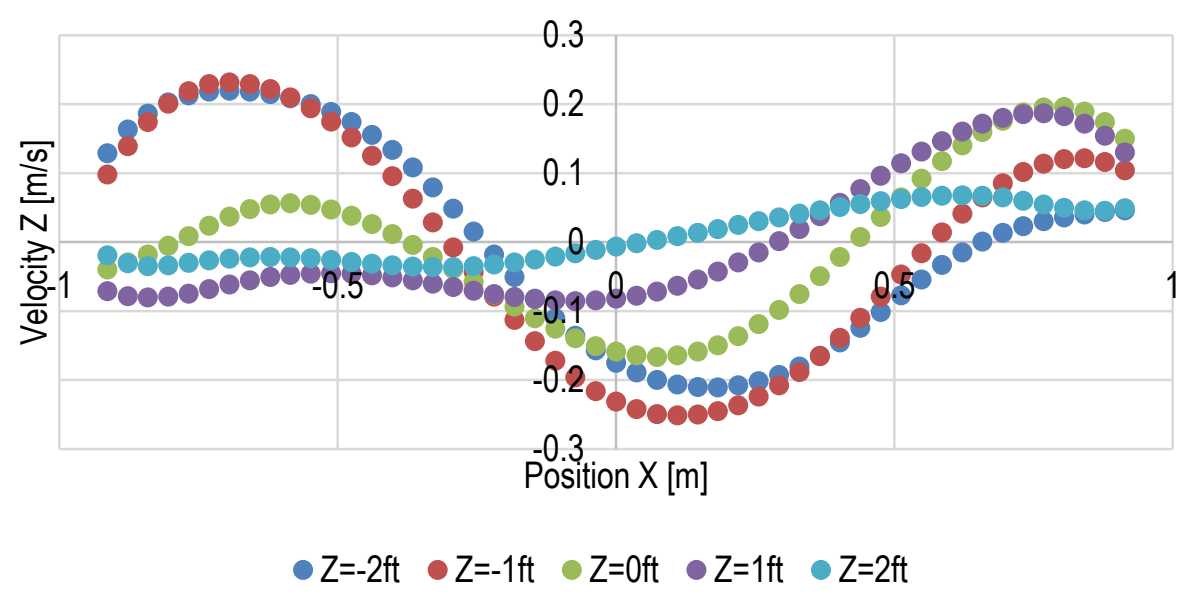

(b)

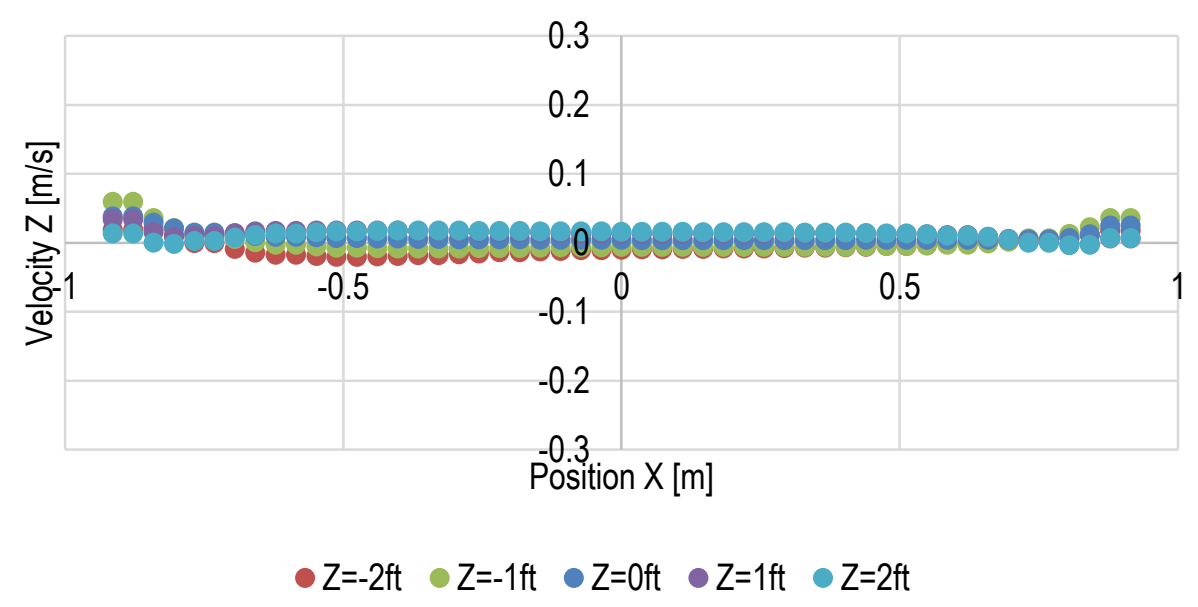

(c)

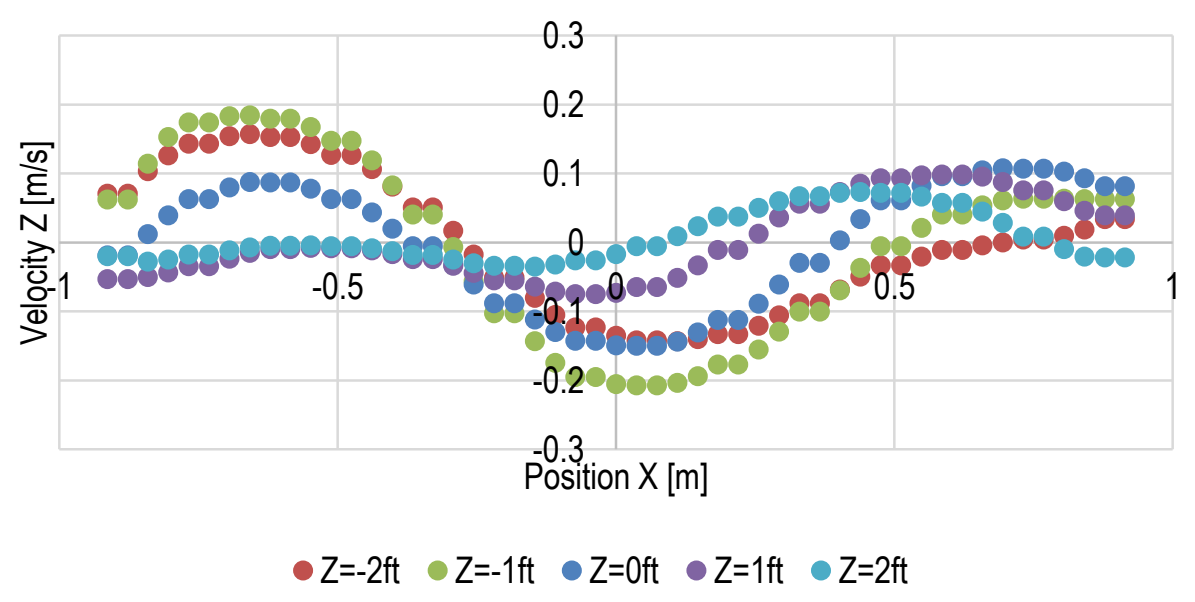

Figure B. 5. Velocity in $Z$ direction on a vertical plane, located half way between the centerline between the force balance plates and the outlet of the wind tunnel nozzle, at fan speed $48 \mathrm{rpm}$, (a) with rotating reference frame, (b) simplified geometry with uniform inlet velocity, (c) simplified geometry with nonuniform inlet 
(a)

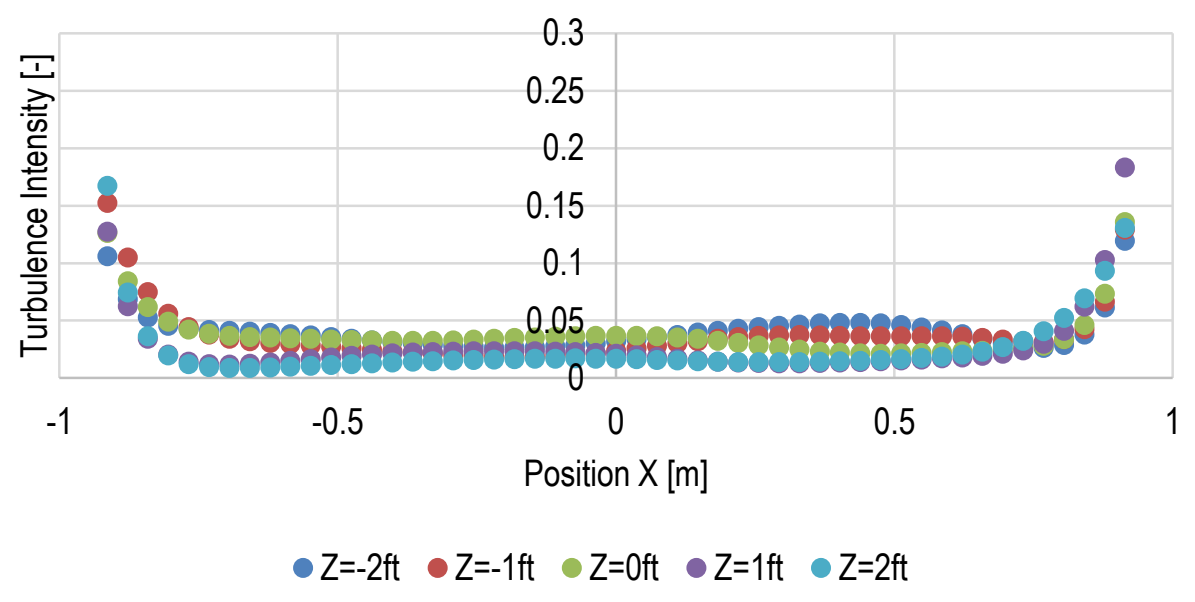

(b)

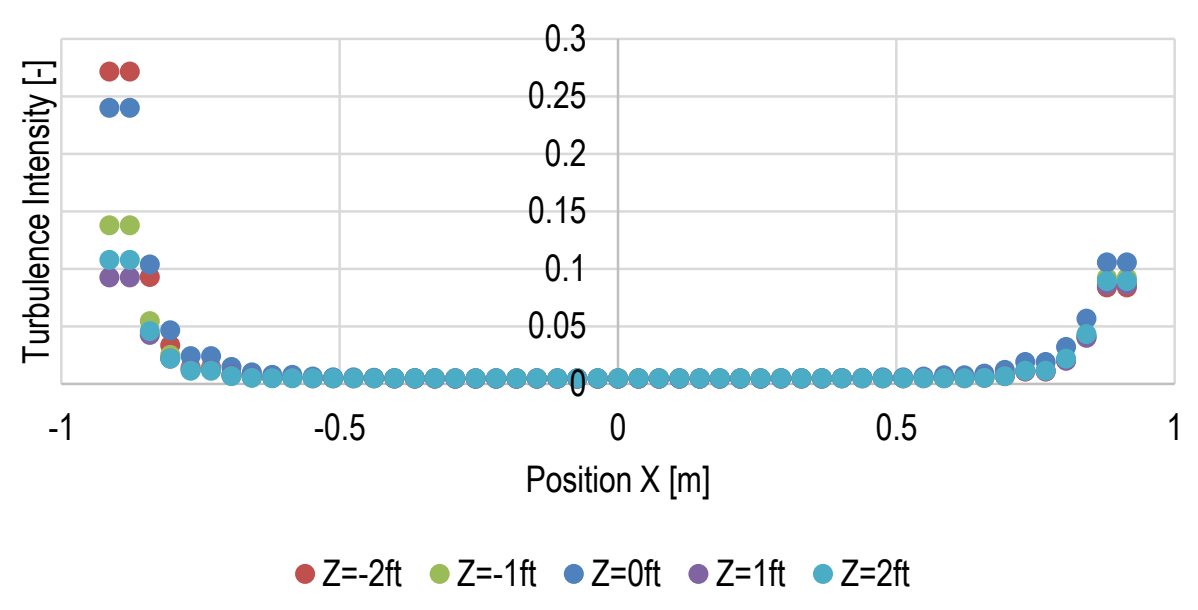

(c)

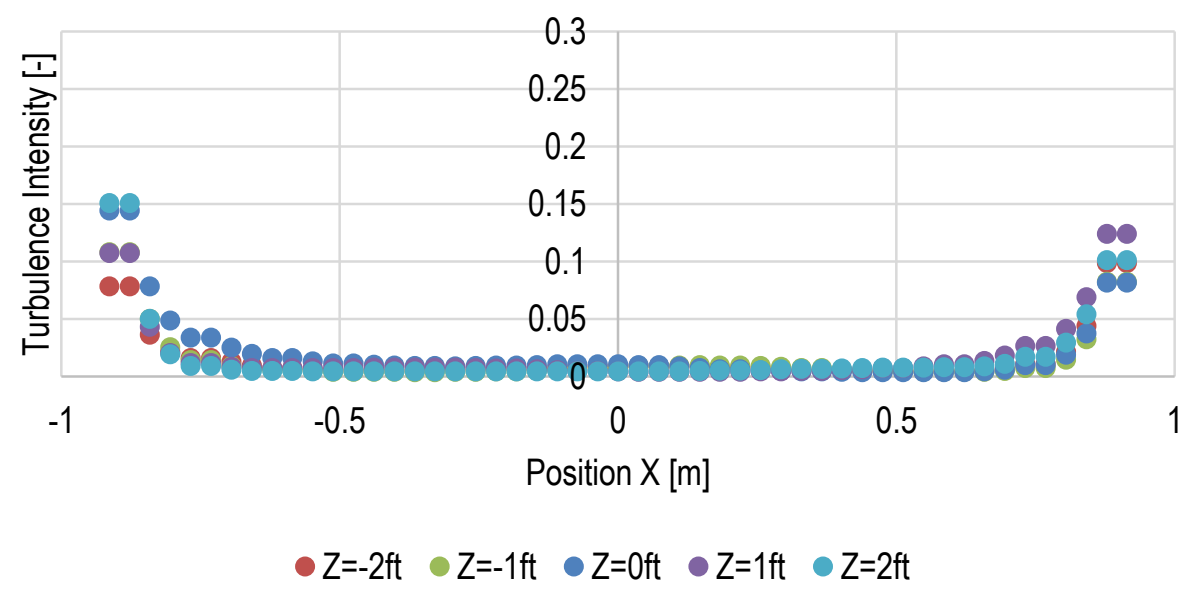

Figure B. 6. Turbulence intensity on a vertical plane, located half way between the centerline between the force balance plates and the outlet of the wind tunnel nozzle, at fan speed $48 \mathrm{rpm}$, (a) with rotating reference frame, (b) simplified geometry with uniform inlet velocity, (c) simplified geometry with nonuniform inlet 
(a)

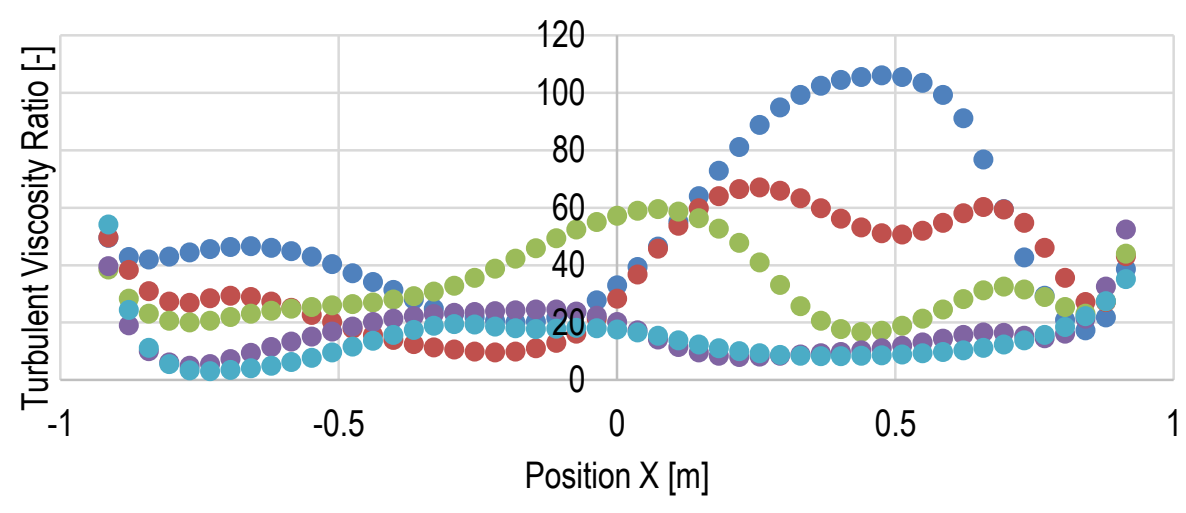

$$
\bullet Z=-2 \mathrm{ft} \quad \mathrm{Z}=-1 \mathrm{ft} \quad \mathrm{Z}=0 \mathrm{ft} \quad \mathrm{Z}=1 \mathrm{ft} \quad \mathrm{Z}=2 \mathrm{ft}
$$

(b)

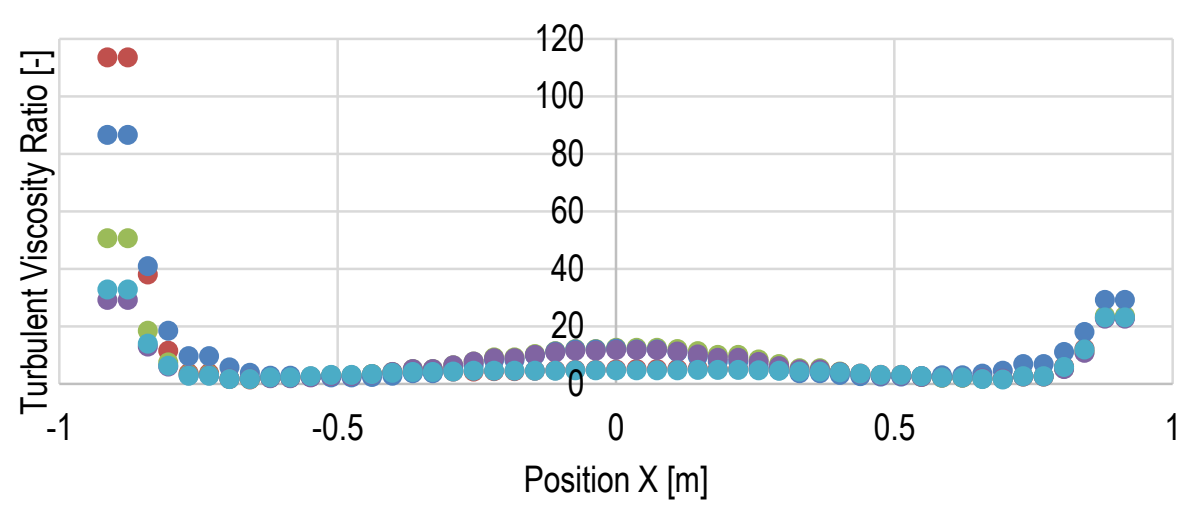

$\bullet Z=-2 \mathrm{ft} \quad \mathrm{Z}=-1 \mathrm{ft} \quad \mathrm{Z}=0 \mathrm{ft} \quad \mathrm{Z}=1 \mathrm{ft} \quad \bullet \mathrm{Z}=2 \mathrm{ft}$

(c)

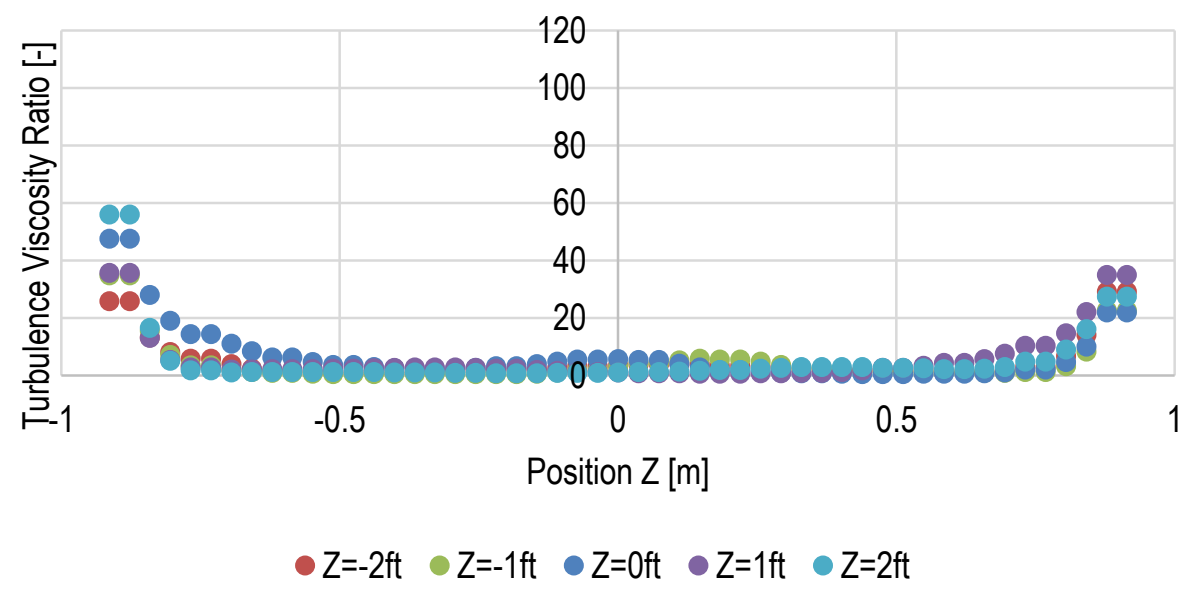

Figure B. 7. Turbulent viscosity ratio on a vertical plane, located half way between the centerline between the force balance plates and the outlet of the wind tunnel nozzle, at fan speed $48 \mathrm{rpm}$, (a) with rotating reference frame, (b) simplified geometry with uniform inlet velocity, (c) simplified geometry with nonuniform inlet 
(a)

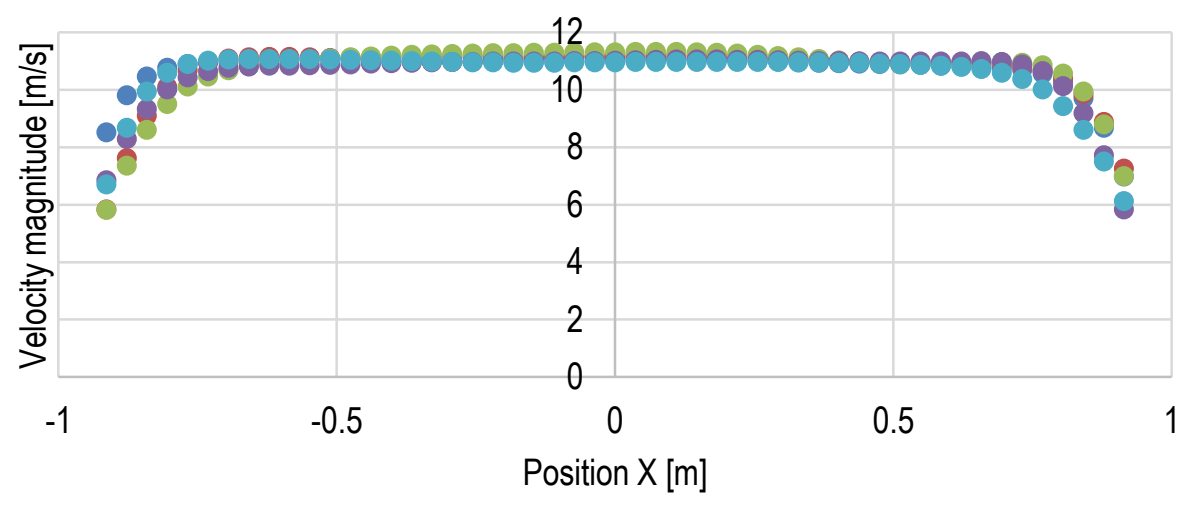

$\bullet Z=-2 \mathrm{ft} \odot Z=-1 \mathrm{ft} \odot \mathrm{Z}=0 \mathrm{ft} \quad \mathrm{Z}=1 \mathrm{ft} \quad \mathrm{Z}=2 \mathrm{ft}$

(b)

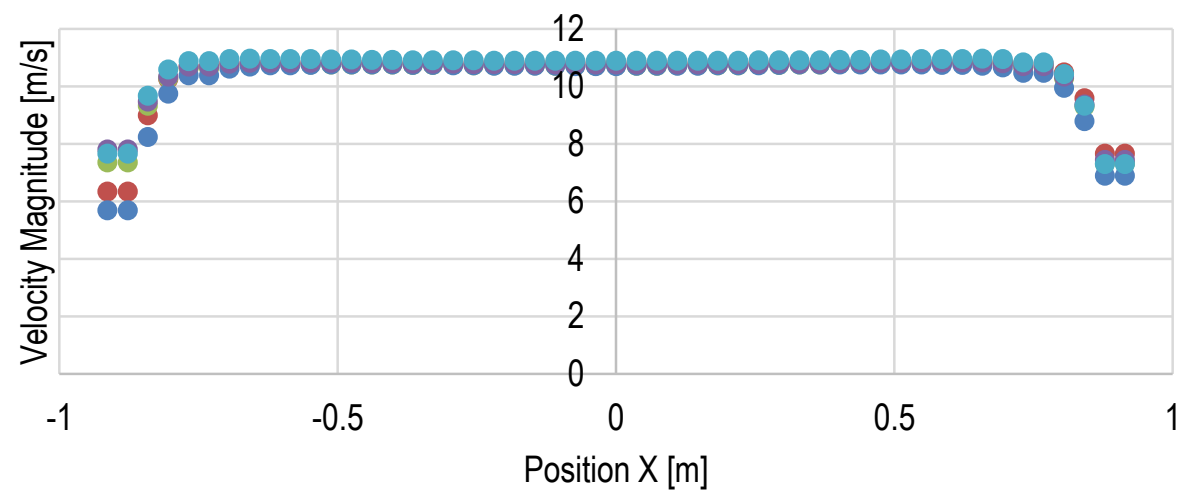

$\circ Z=-2 f t \quad Z=-1 f t \quad Z Z=0 f t \quad O Z=1 f t \quad \circ Z=2 f t$

(c)

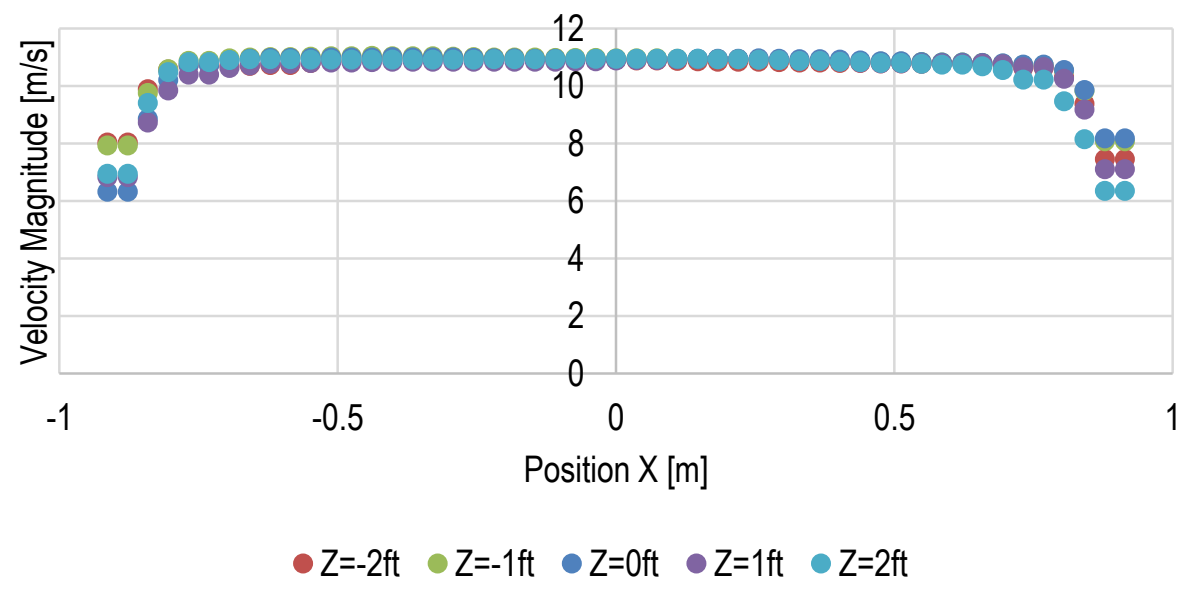

Figure B. 8. Velocity magnitude on a vertical plane, located half way between the centerline between the force balance plates and the outlet of the wind tunnel nozzle, at fan speed $437 \mathrm{rpm}$, (a) with rotating reference frame, (b) simplified geometry with uniform inlet velocity, (c) simplified geometry with nonuniform inlet velocity 
(a)

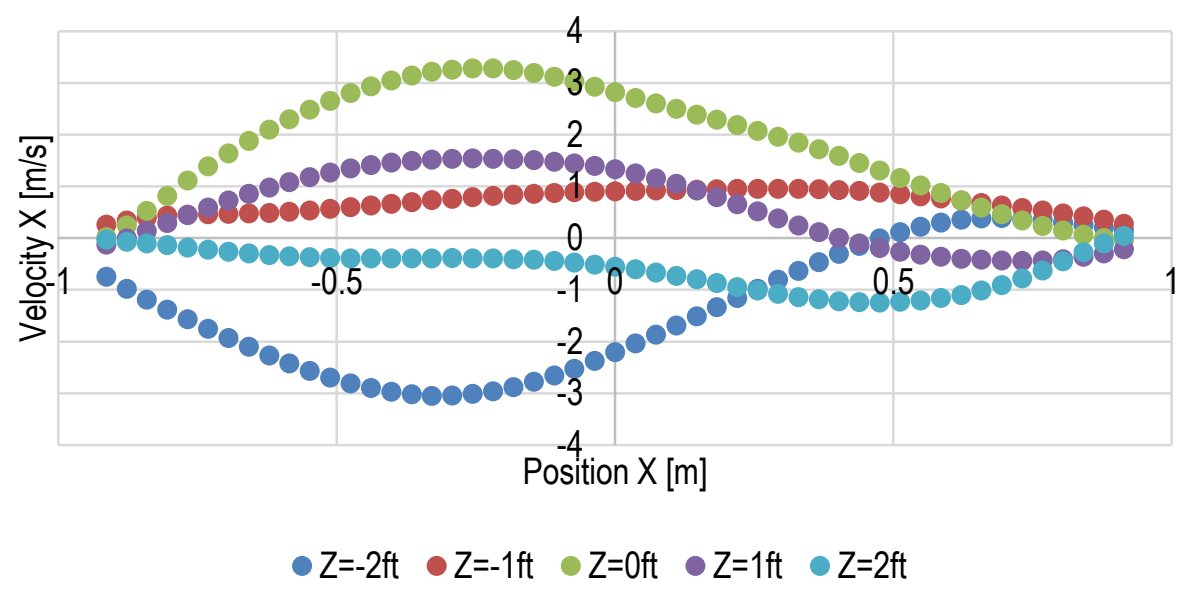

(b)

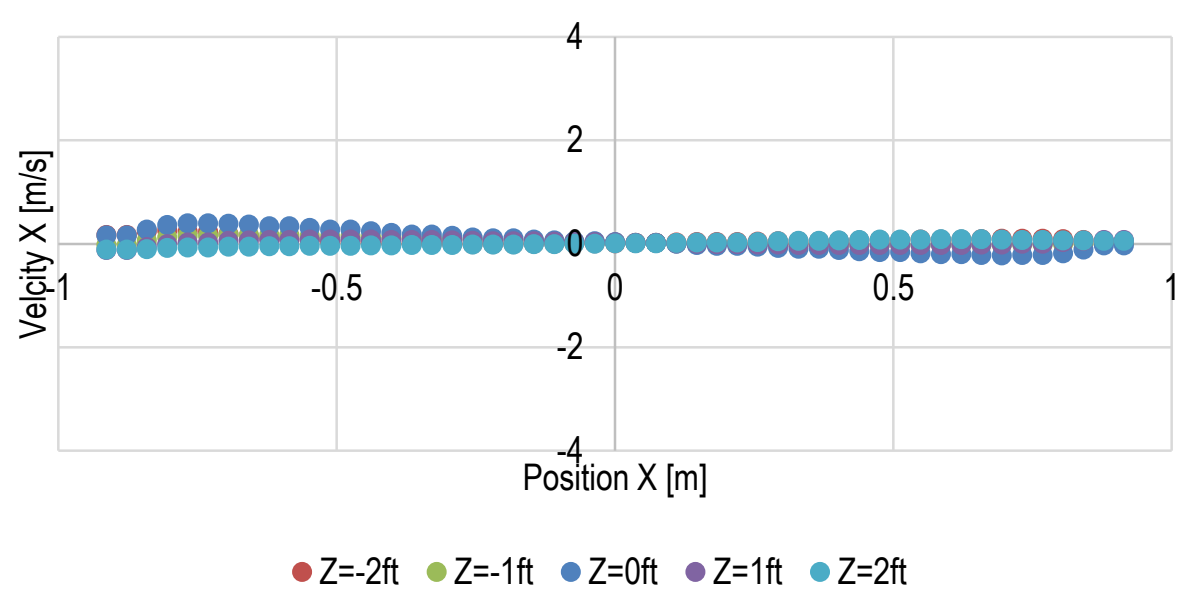

(c)

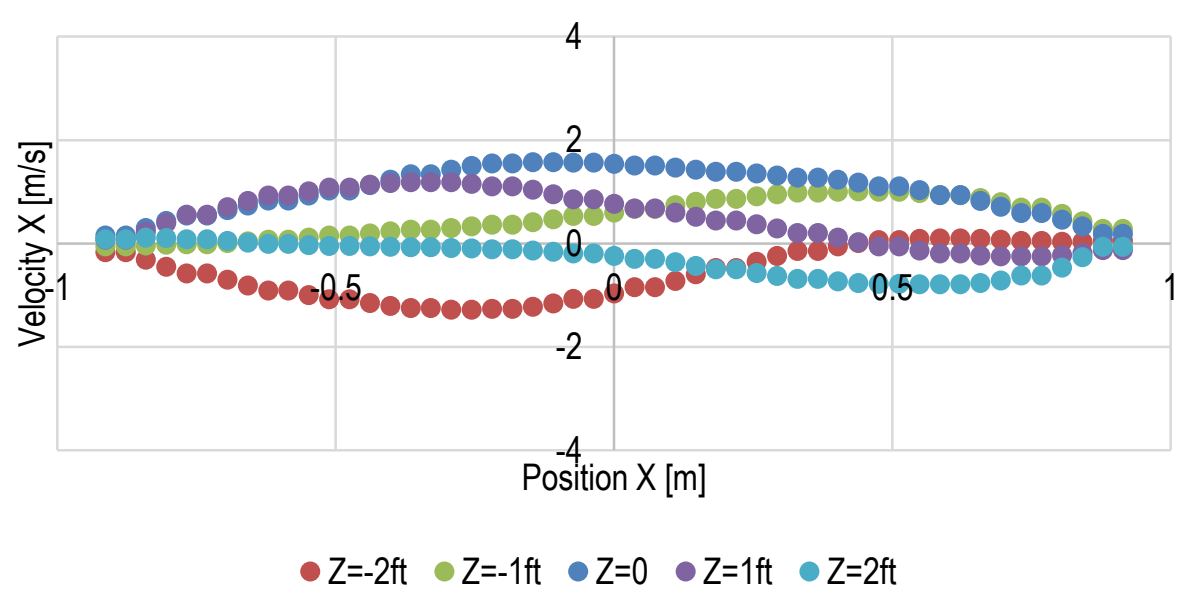

Figure B. 9. Velocity in $\mathrm{X}$ direction on a vertical plane located half way between the centerline between the force balance plates and the outlet of the wind tunnel nozzle, at fan speed $437 \mathrm{rpm}$, (a) with rotating reference frame, (b) simplified geometry with uniform inlet velocity, (c) simplified geometry with nonuniform inlet velocity 
(a)

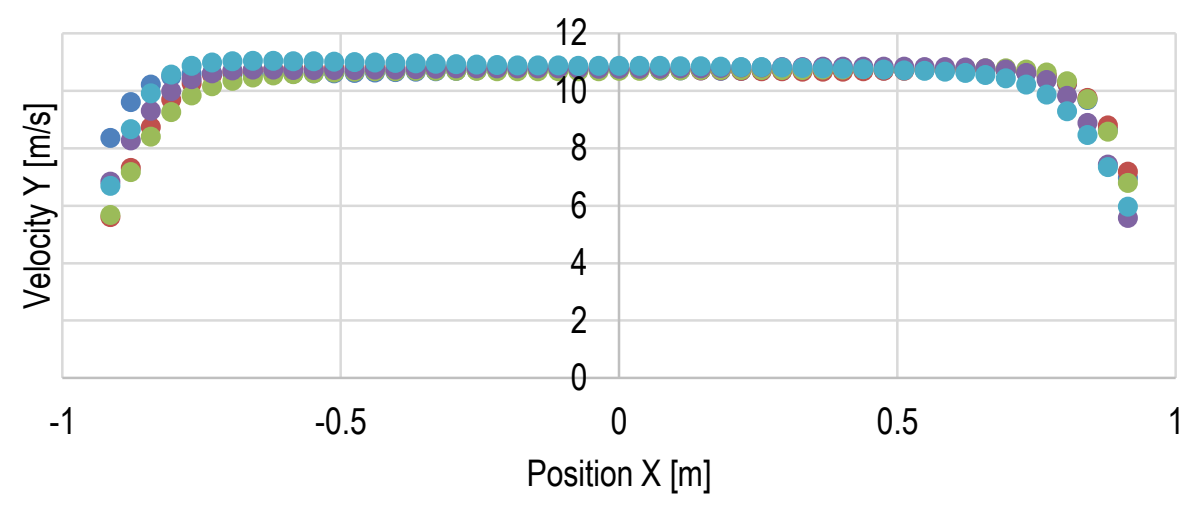

$\cdot Z=-2 \mathrm{ft} \cdot Z=-1 \mathrm{ft} \odot Z=0 \mathrm{ft} \cdot Z=1 \mathrm{ft} \cdot Z=2 \mathrm{ft}$

(b)

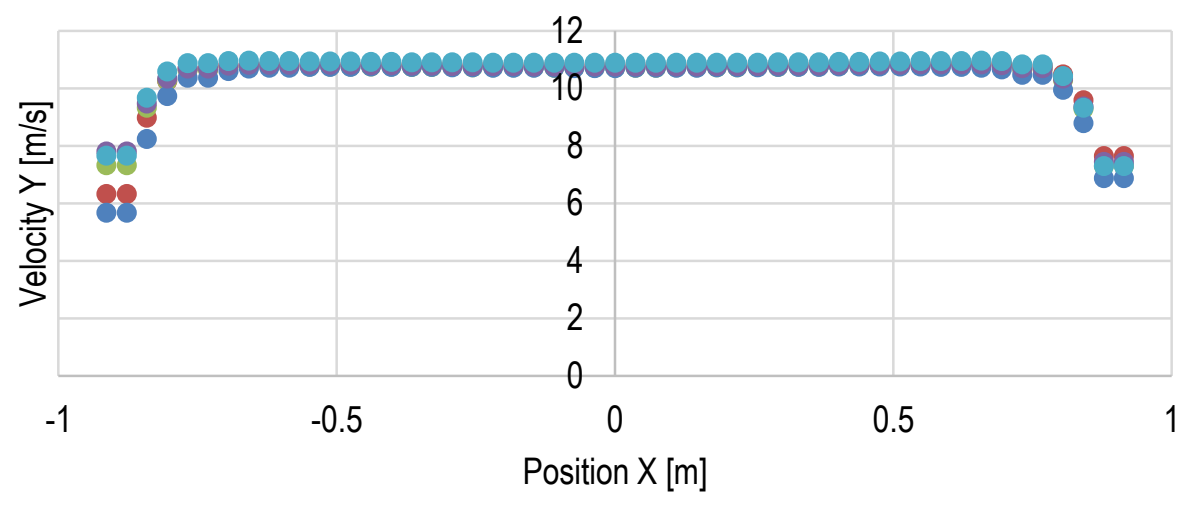

$\bullet Z=-2 f t \quad Z=-1 \mathrm{ft} \odot Z=0 f t \quad Z=1 \mathrm{ft} \odot Z=2 \mathrm{ft}$

(c)

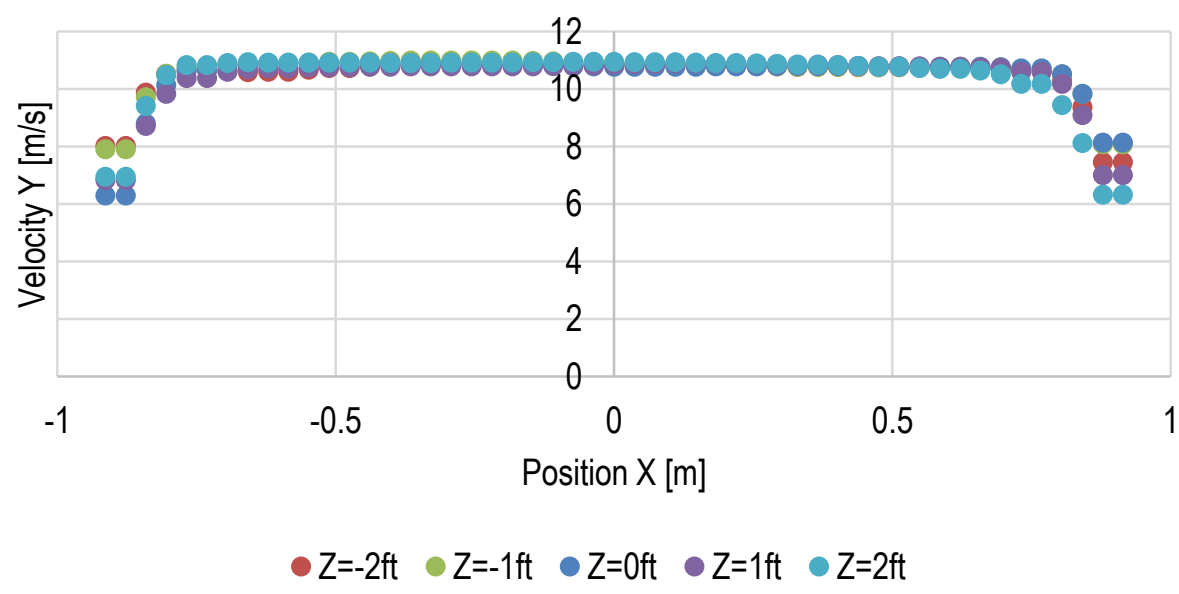

Figure B. 10. Velocity in Y direction on a vertical plane, located half way between the centerline between the force balance plates and the outlet of the wind tunnel nozzle, at fan speed $437 \mathrm{rpm}$, (a) with rotating reference frame, (b) simplified geometry with uniform inlet velocity, (c) simplified geometry with nonuniform inlet 
(a)

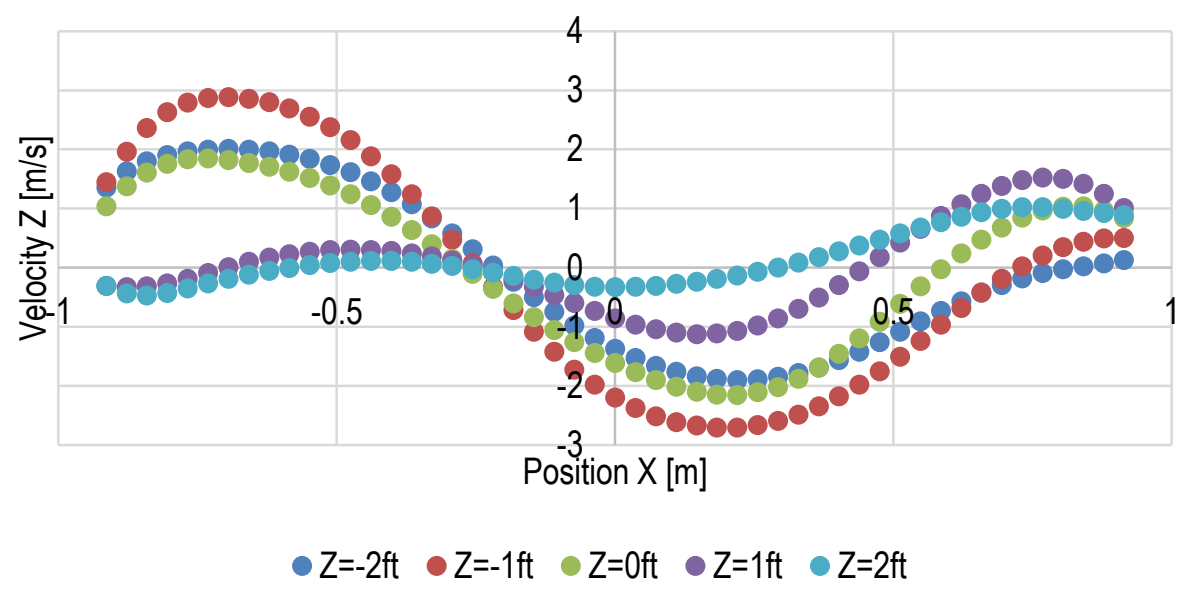

(b)

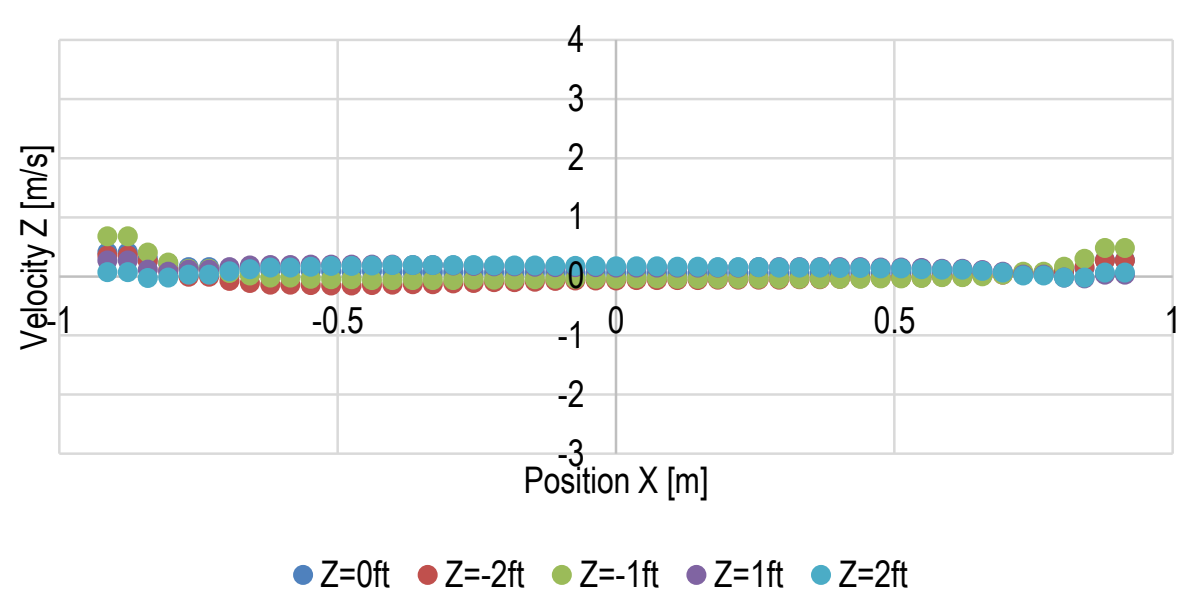

(c)

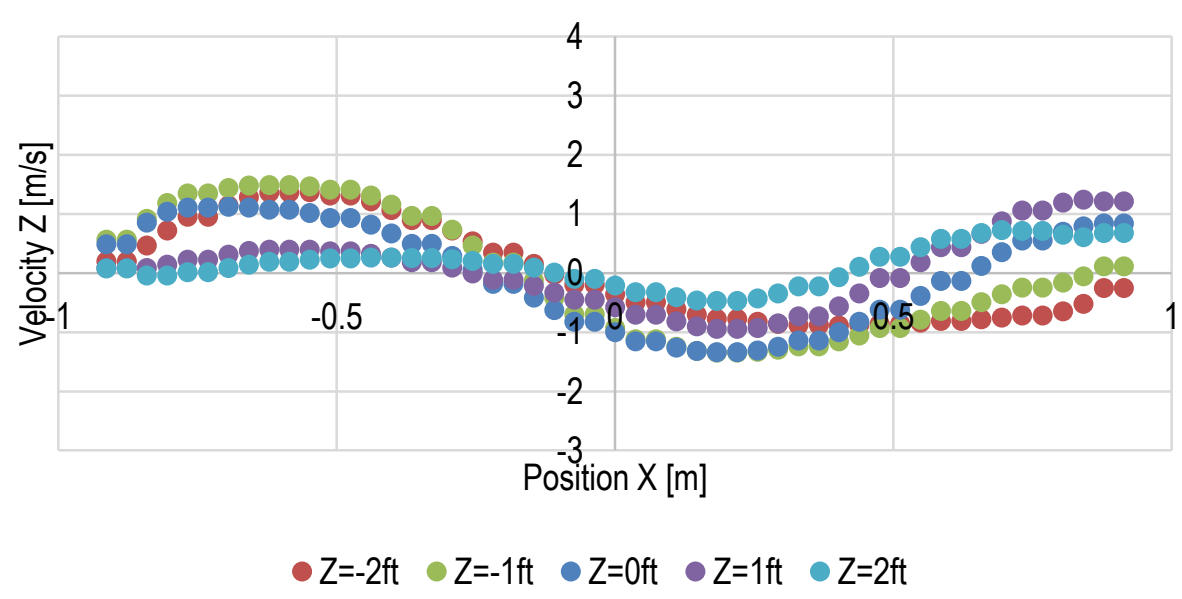

Figure B. 11. Velocity in $Z$ direction on a vertical plane, located half way between the centerline between the force balance plates and the outlet of the wind tunnel nozzle, at fan speed $437 \mathrm{rpm}$, (a) with rotating reference frame, (b) simplified geometry with uniform inlet velocity, (c) simplified geometry with nonuniform inlet 
(a)

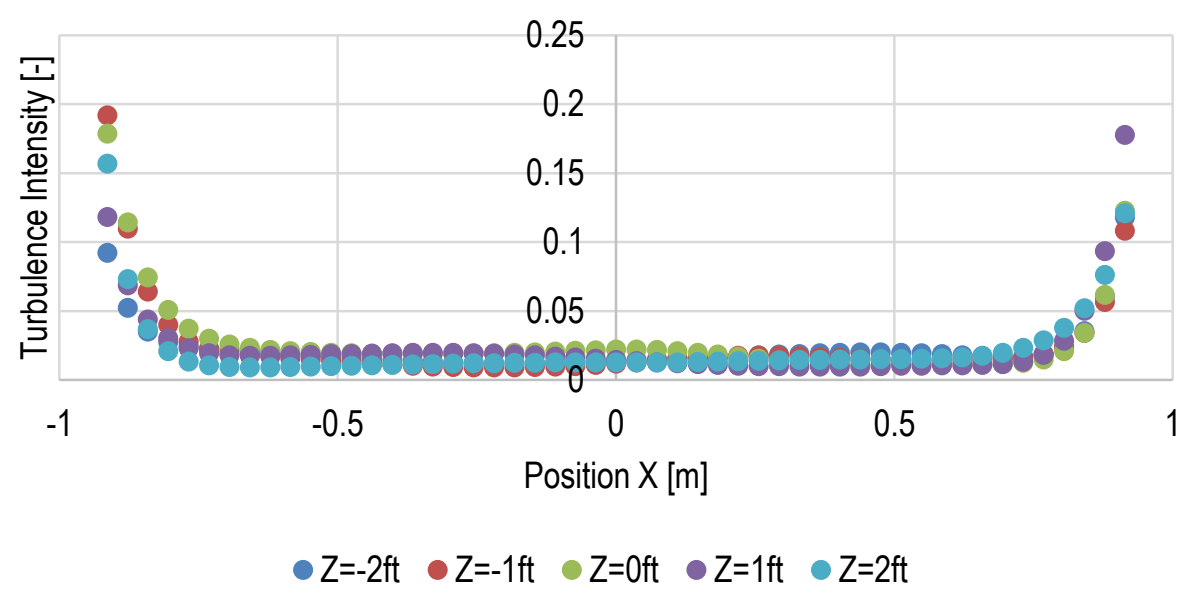

(b)

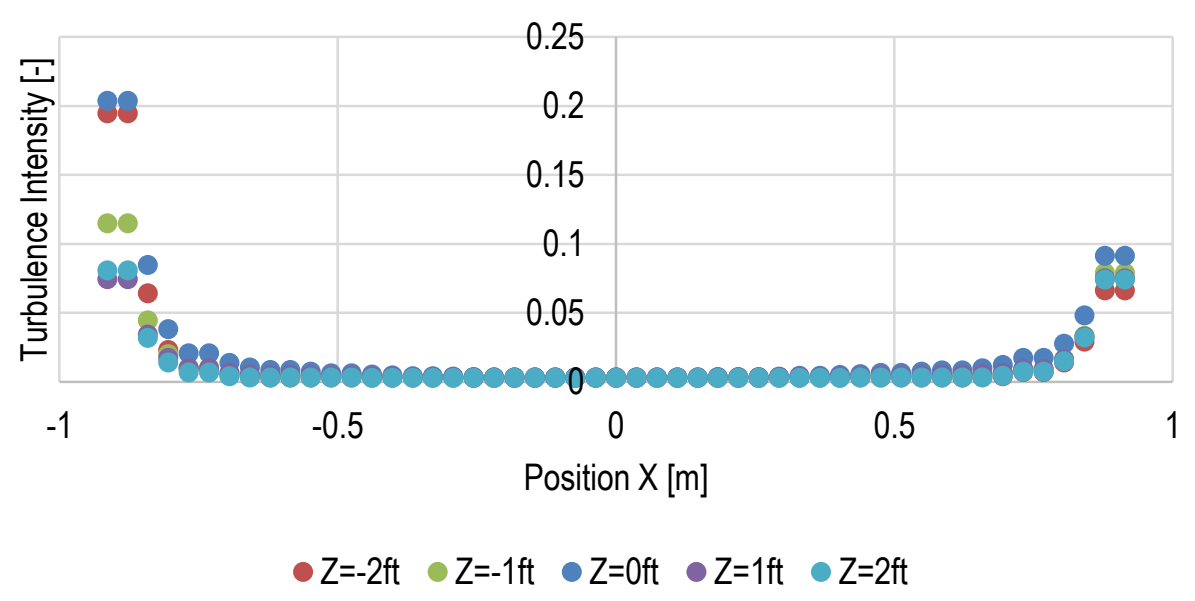

(c)

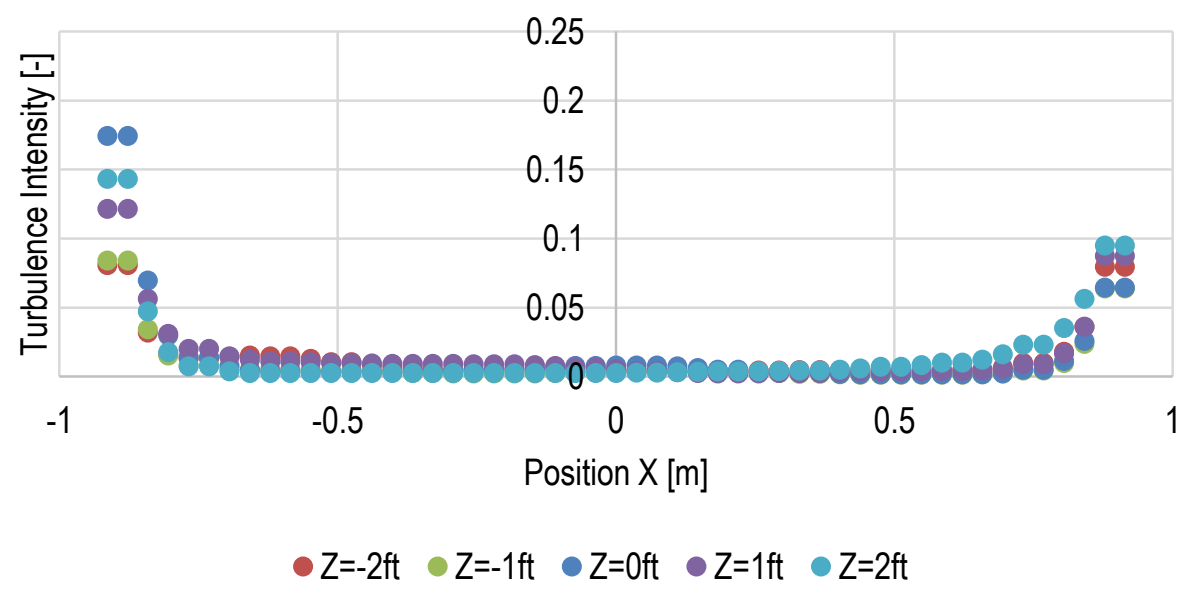

Figure B. 12. Turbulence intensity on a vertical plane, located half way between the centerline between the force balance plates and the outlet of the wind tunnel nozzle, at fan speed $437 \mathrm{rpm}$, (a) with rotating reference frame, (b) simplified geometry with uniform inlet velocity, (c) simplified geometry with nonuniform inlet 
(a)

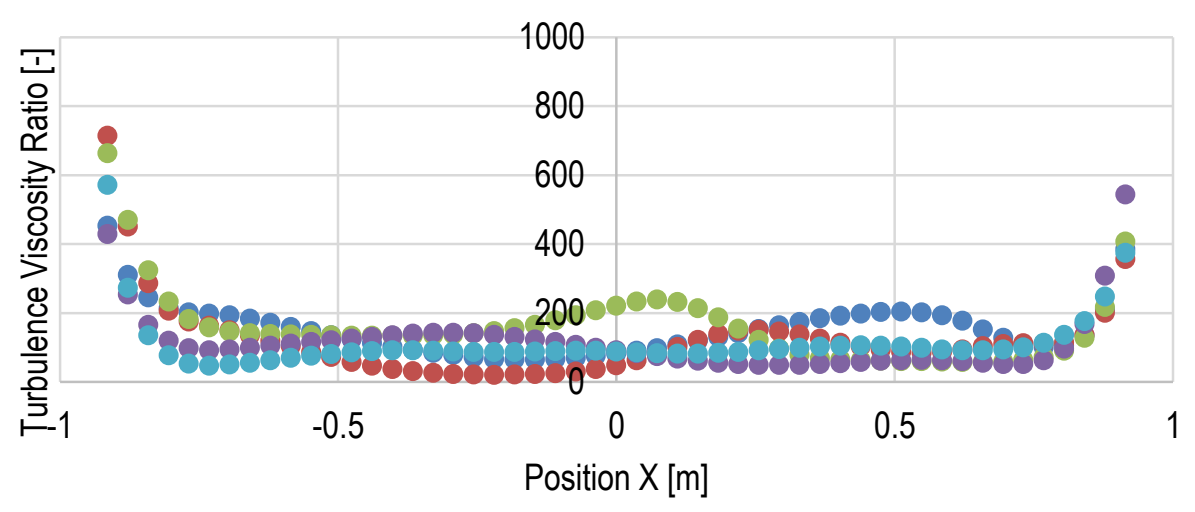

$\bullet Z=-2 \mathrm{ft} \cdot Z=-1 \mathrm{ft} \odot Z=0 \mathrm{ft} \cdot \mathrm{Z}=1 \mathrm{ft} \cdot \mathrm{Z}=2 \mathrm{ft}$

(b)

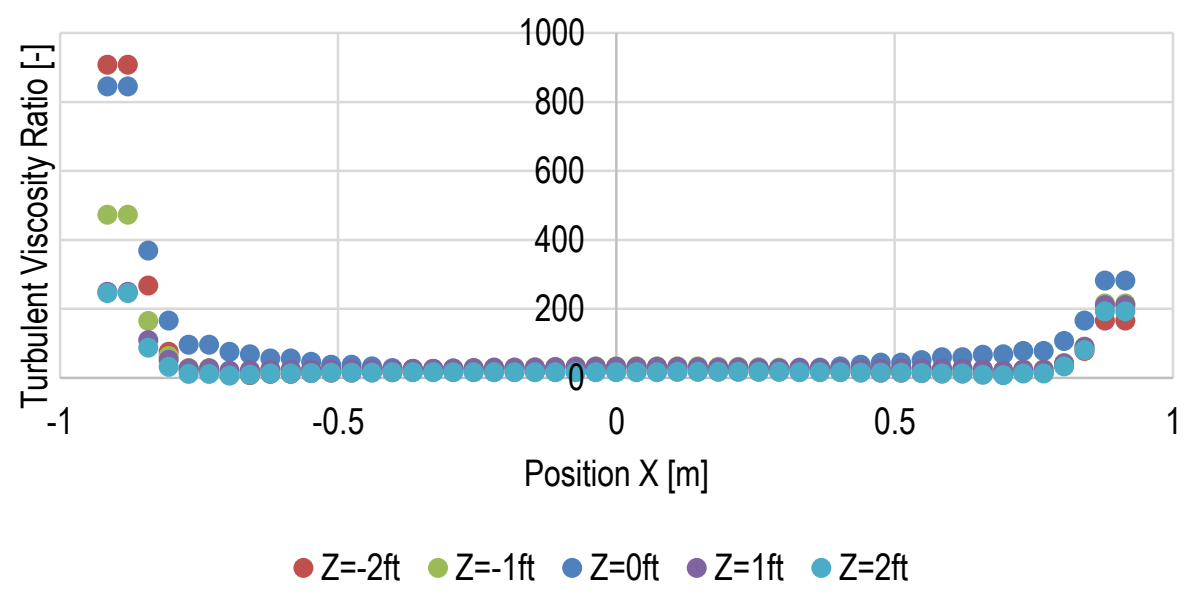

(c)

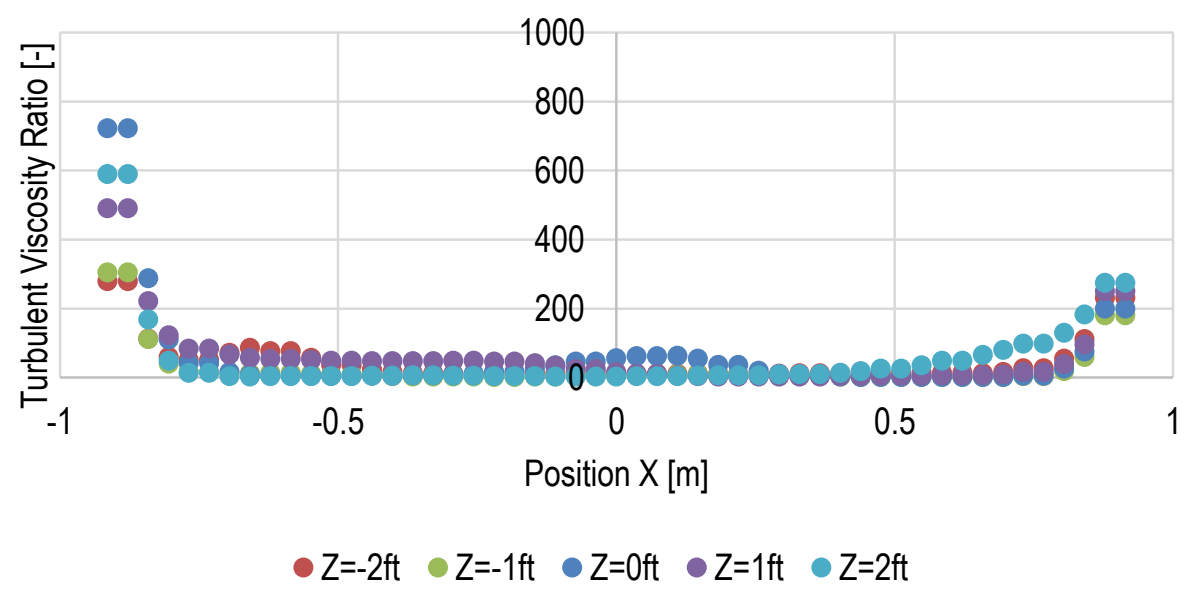

Figure B. 13. Turbulent viscosity ratio on a vertical plane, located half way between the centerline between the force balance plates and the outlet of the wind tunnel nozzle, at fan speed $437 \mathrm{rpm}$, (a) with rotating reference frame, (b) simplified geometry with uniform inlet velocity, (c) simplified geometry with nonuniform inlet 
(a)

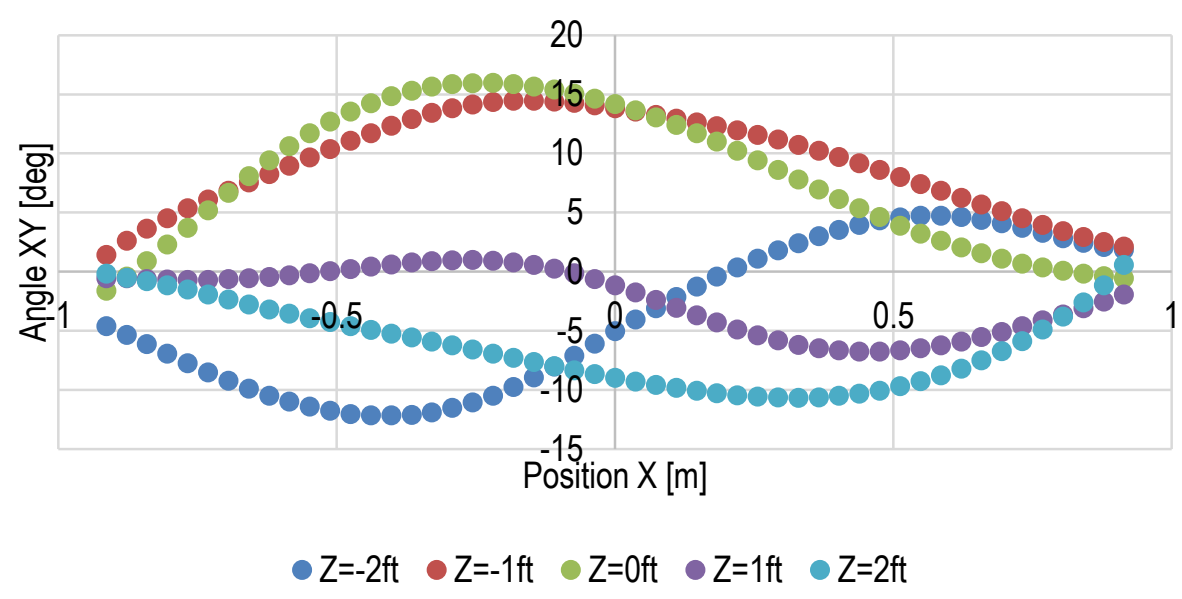

(b)

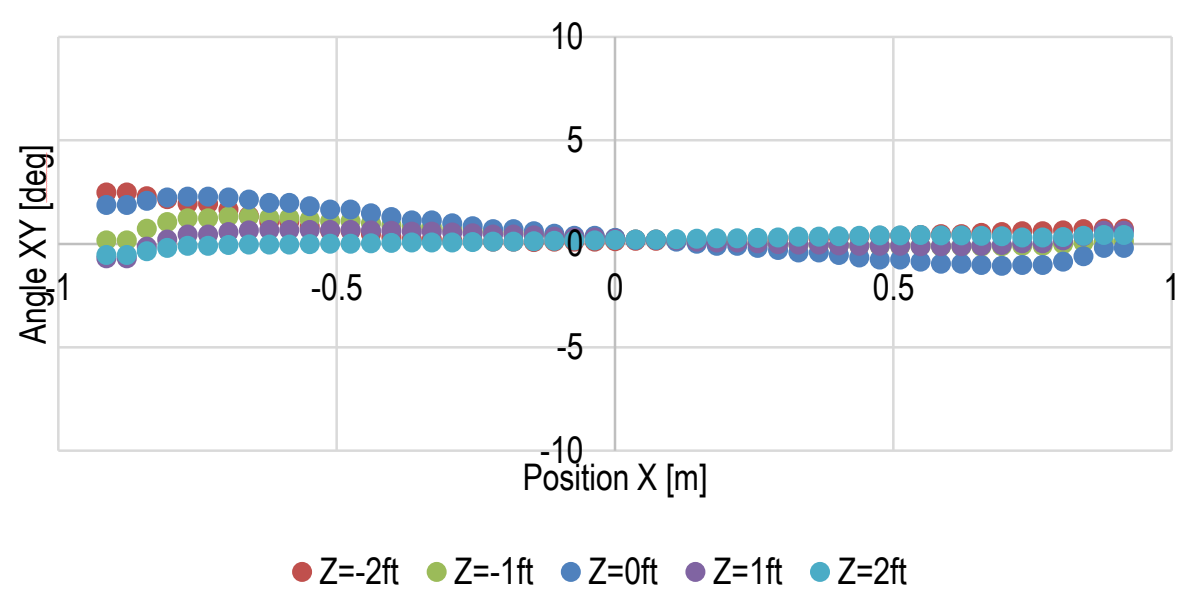

(c)

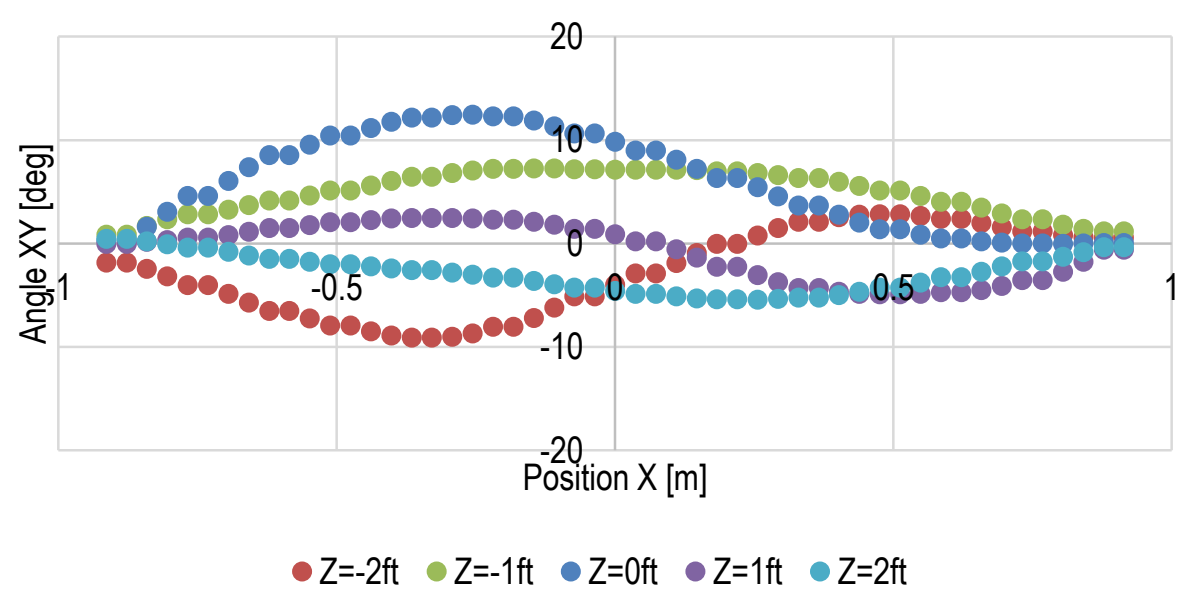

Figure B. 14. XY angle of velocity at points on a vertical plane, located half way between the centerline between the force balance plates and the outlet of the wind tunnel nozzle, at fan speed $48 \mathrm{rpm}$, in models:

(a) with rotating reference frame, (b) simplified geometry with uniform inlet velocity, (c) simplified geometry with non-uniform inlet 
(a)

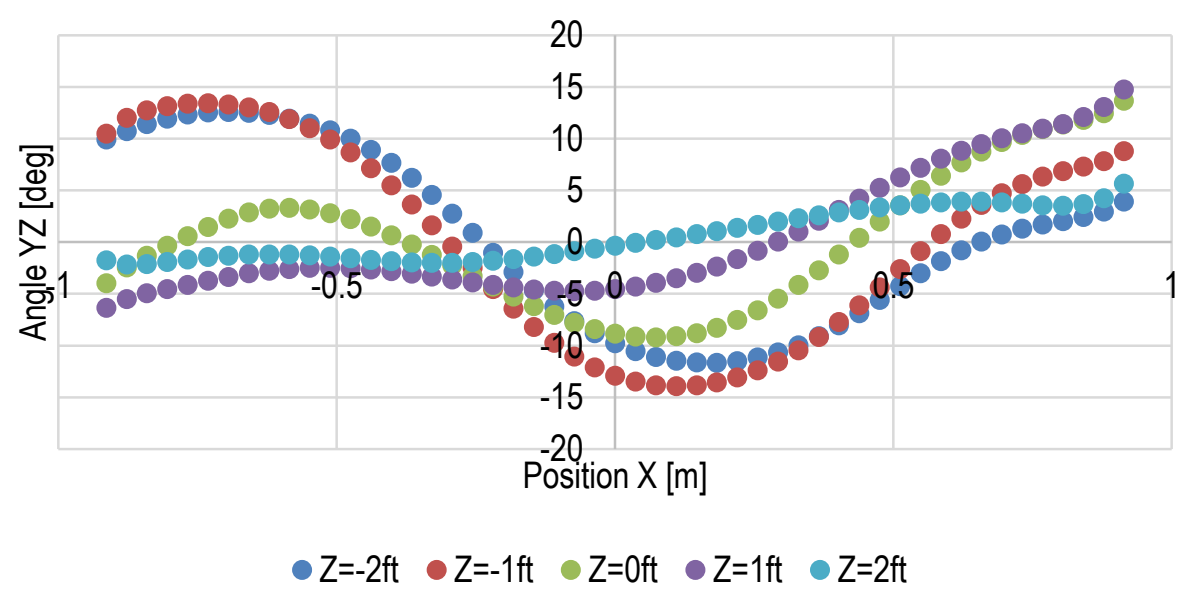

(b)

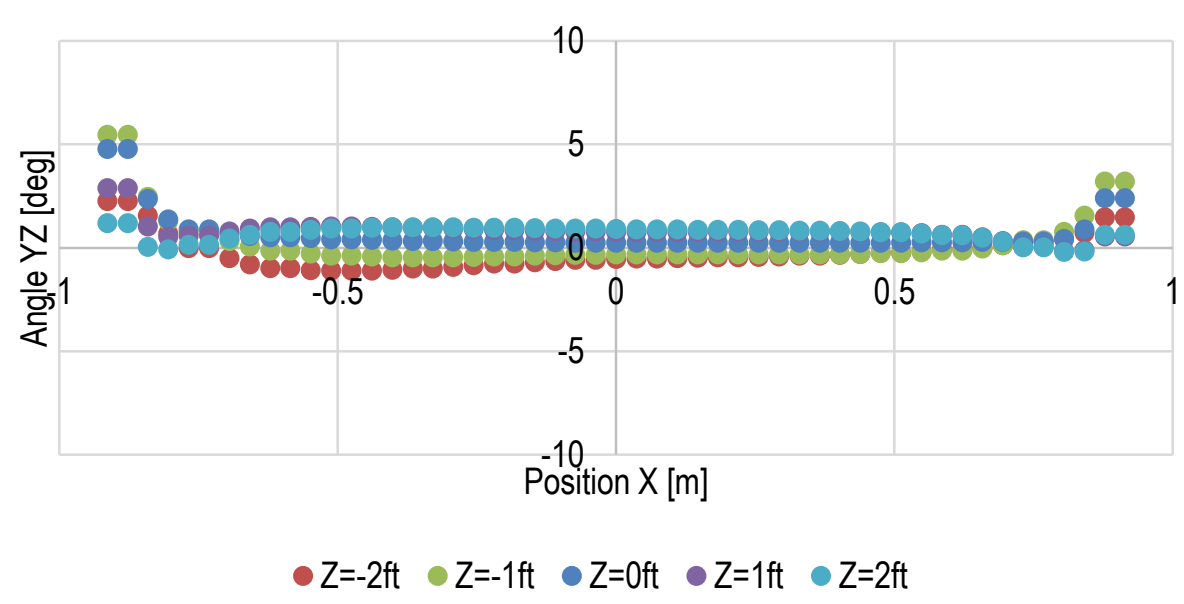

(c)

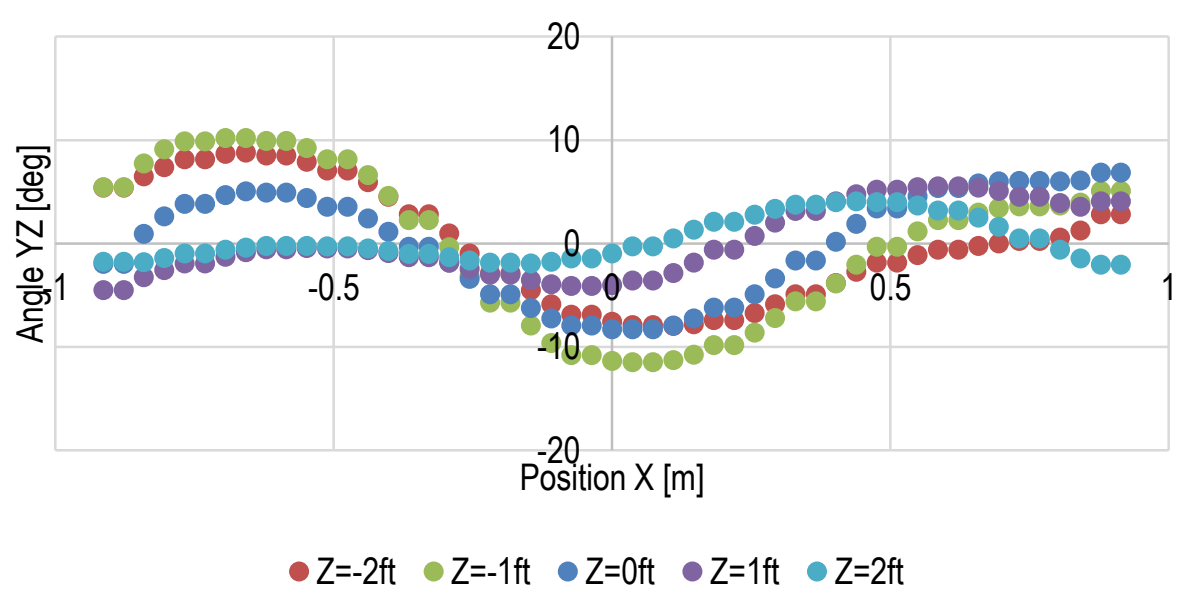

Figure B. 15. XZ angle of velocity at points on a vertical plane, located half way between the centerline between the force balance plates and the outlet of the wind tunnel nozzle, at fan speed $48 \mathrm{rpm}$, in models:

(a) with rotating reference frame, (b) simplified geometry with uniform inlet velocity, (c) simplified geometry with non-uniform inlet 
(a)

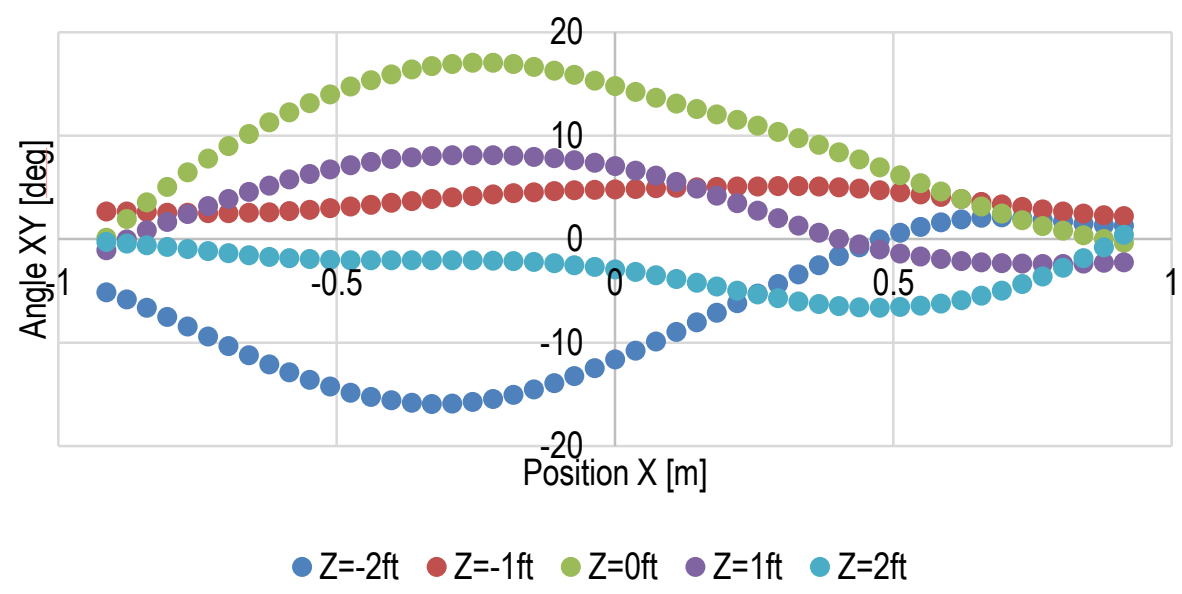

(b)

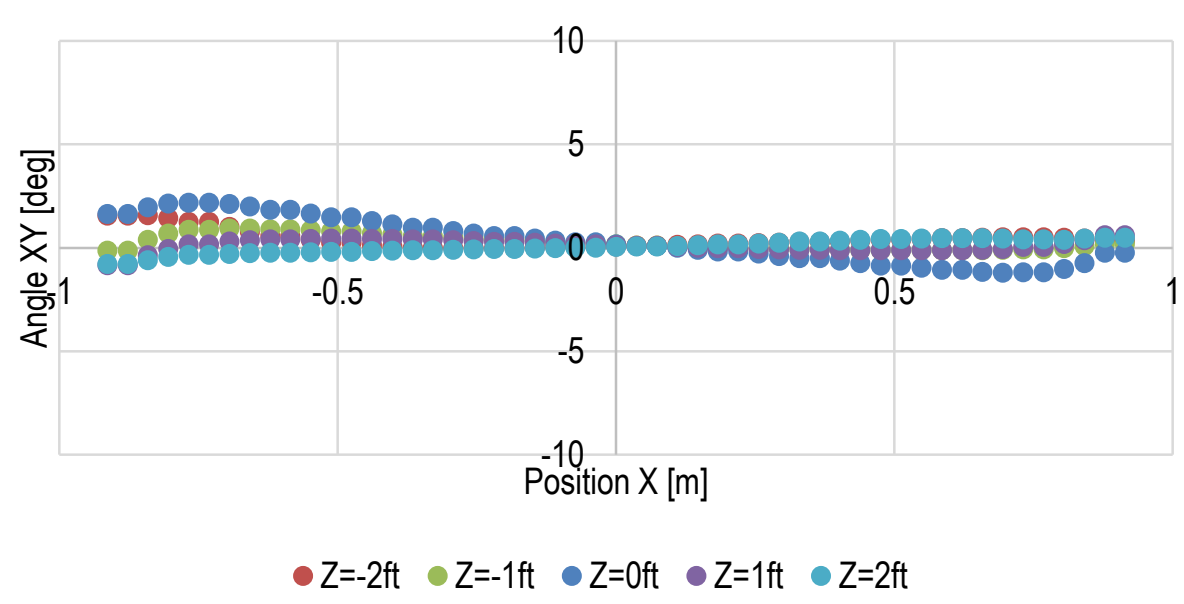

(c)

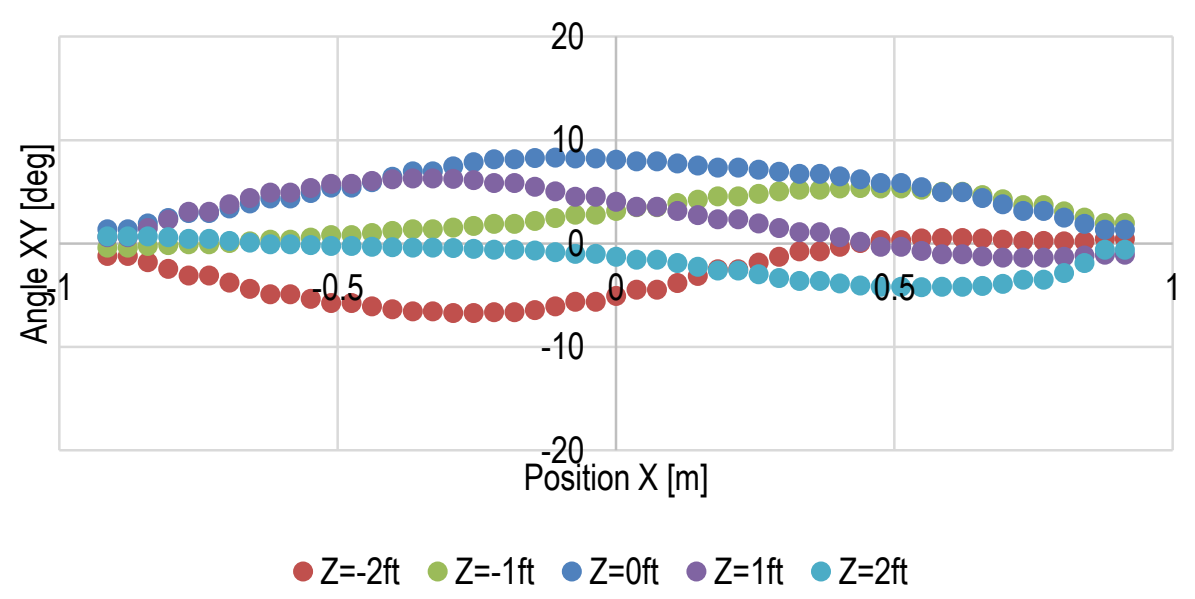

Figure B. 16. XY angle of velocity at points on a vertical plane, located half way between the centerline between the force balance plates and the outlet of the wind tunnel nozzle, at fan speed $437 \mathrm{rpm}$, in models: (a) with rotating reference frame, (b) simplified geometry with uniform inlet velocity, (c) simplified geometry with non-uniform inlet 
(a)

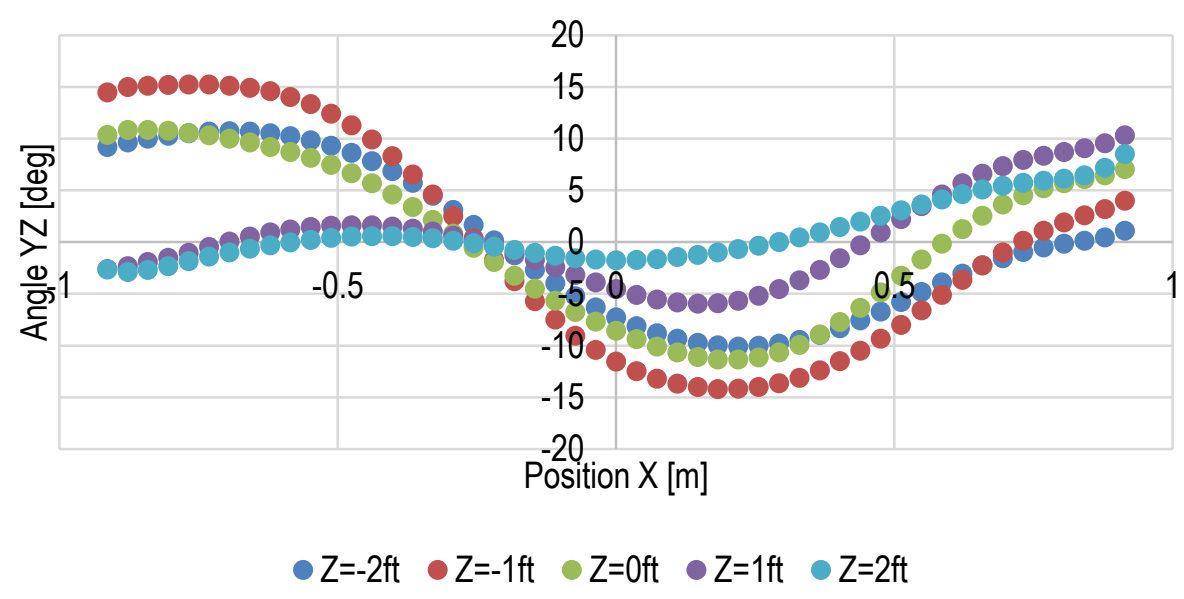

(b)

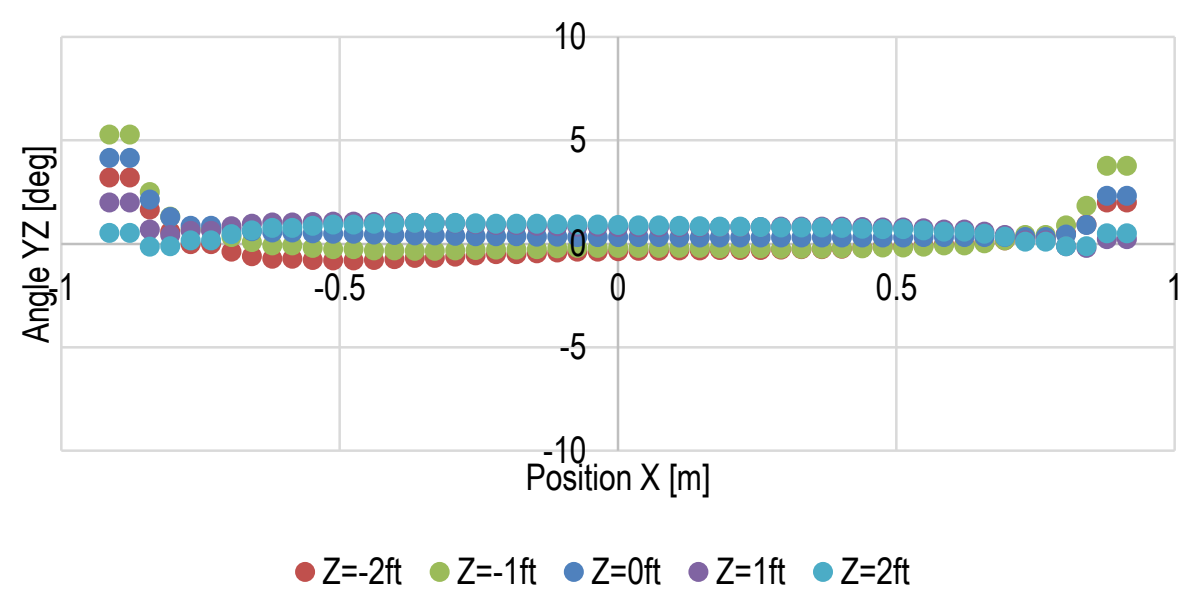

(c)

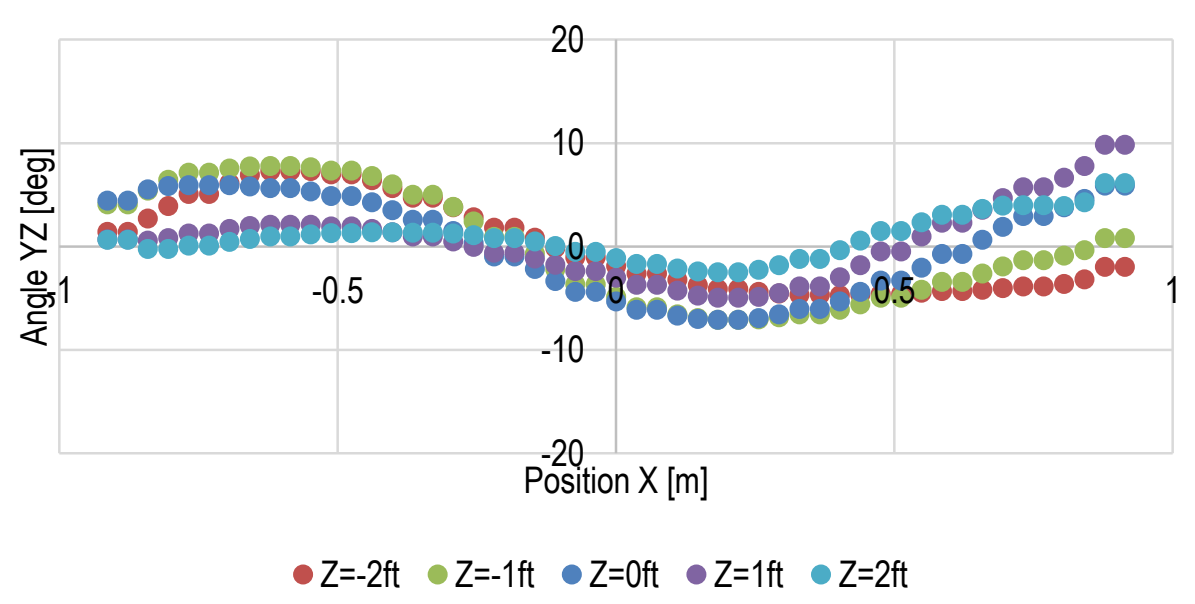

Figure B. 17. XZ angle of velocity at points on a vertical plane, located half way between the centerline between the force balance plates and the outlet of the wind tunnel nozzle, at fan speed $437 \mathrm{rpm}$, in models: (a) with rotating reference frame, (b) simplified geometry with uniform inlet velocity, (c) simplified geometry with non-uniform inlet 


\section{Argonne}

Nuclear Engineering Division

Argonne National Laboratory

9700 South Cass Avenue, bldg. 208

Argonne, IL 60439-4815

www.anl.gov

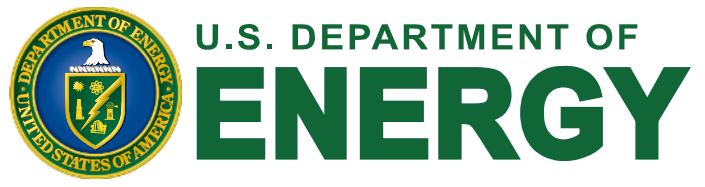

Argonne National Laboratory is a U.S. Department of Energy laboratory managed by UChicago Argonne, LLC

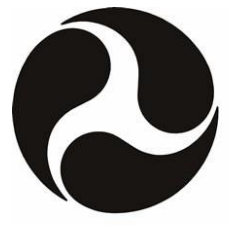

\section{U.S. Department of Transportation Federal Highway Administration}

